\title{
Mykotoxine und Kindergesundheit
}

\section{Elterliche Risikowahrnehmung und neue Ansätze für das Risikomanagement}

Dissertation zur Erlangung des Doktorgrades der Fakultät für Agrarwissenschaften der Georg-August-Universität Göttingen

Vorgelegt von Christine Niens

geboren in Wolfenbüttel 
D7

1. Referentin/Referent: Prof. Dr. Rainer Marggraf 2. Korreferentin/Korreferent: PD Dr. Micha Strack

Tag der mündlichen Prüfung: 05.07.2013 
Inhalt

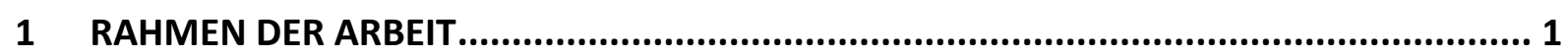

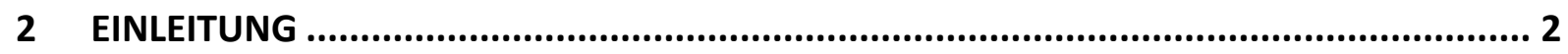

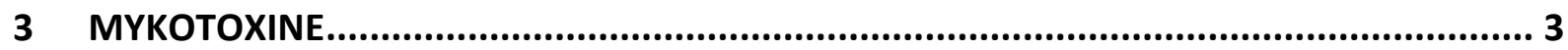

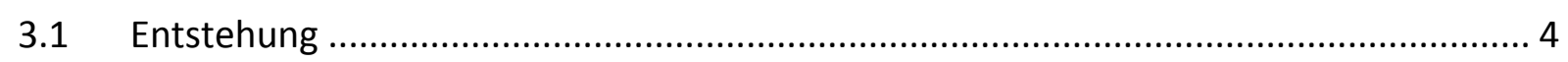

3.2 Potential zur Schädigung der Gesundheit .............................................................. 5

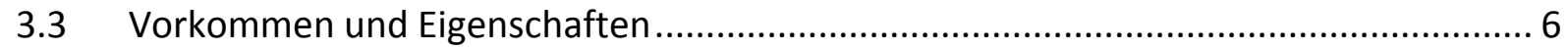

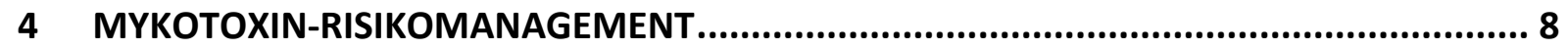

4.1 Aktuelle Maßnahmen des gesundheitlichen Verbraucherschutzes vor Mykotoxinen...9

4.2 Defizite der aktuellen Verbraucherschutzmaßnahmen und Risiken für den

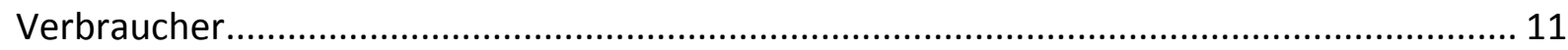

4.3 Mykotoxin-Risikowahrnehmung aus Konsumentensicht ....................................... 13

4.4 Grundlagen der wissenschaftlichen Klassifizierung von Risiken entsprechend des

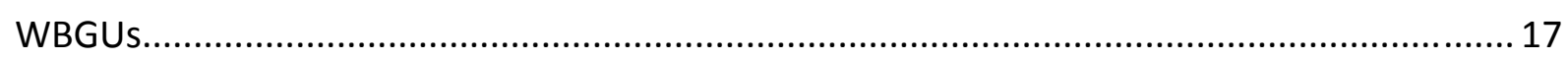

4.4.1 Einordnung des DON-Risikos in die Risikotypologie des WBGUs ...................................................20

4.4.2 Ableitung geeigneter Verbesserungsansätze für das DON-Risikomanagement zur Verbesserung des

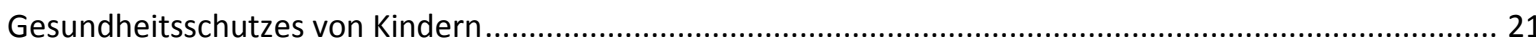

5 GRUNDLEGENDE FRAGESTELLUNGEN UND ZIELSETZUNG DER ARBEIT ...................... 22

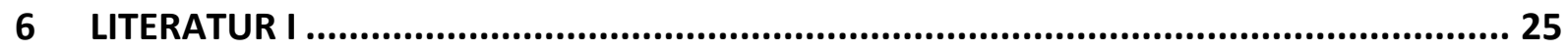

7 GLIEDERUNG UND INHALT DER WISSENSCHAFTLICHEN VERÖFFENTLICHUNGEN ZUM THEMA „MYKOTOXINE UND KINDERGESUNDHEIT“ .................................................. 31

7.1 Ist eine Verbesserung des Verbraucherschutzes vor Mykotoxinen ökonomisch rational? 
7.2 Ökonomische Bewertung von Kindergesundheit in der Umweltpolitik - Aktuelle Ansätze und ihre Grenzen

7.3 Mykotoxine als Gesundheitsrisiko für Kinder - Regulierungspräferenzen aus

Elternsicht.

7.4 Mykotoxine und Kindergesundheit - Risikowahrnehmung und Zahlungsbereitschaft für eine Risikoreduzierung aus Elternsicht.

7.5 Parental risk perception of mycotoxins and risk reduction behaviour.......

8 DISKUSSION UND AUSBLICK.

9 LITERATUR II

10 ANHANG.. 50

11 LEBENSLAUF 51

12 ERKLÄRUNG 52 


\section{Tabellen und Abbildungen}

\section{Tabellen}

Tabelle 1: Die wichtigsten Mykotoxine in der menschliche Ernährung und ihre jeweiligen Produzenten

Tabelle 2: Deoxynivalenol (DON)-Gehalte in Weizen in Deutschland 2004-2011, angegeben in $\mu \mathrm{g} / \mathrm{kg}$ lufttrockenes Getreide. 7

Tabelle 3: Maximal zulässige Höchstgehalte in $\mu \mathrm{g} / \mathrm{kg}$ Nahrungsmittel für Deoxynivalenol (DON) in Getreide und Getreideprodukten, die für den menschlichen Verzehr bestimmt sind 10

Tabelle 4: Risikocharakteristika, die die Laien-Wahrnehmung eines Risikos beschreiben, zusammengefasst zu drei Faktoren.

Tabelle 5: Wichtige Kriterien für die wissenschaftliche Klassifizierung von Risiken entsprechend des WBGUs (1999).

Tabelle 6: Überblick über die Risikotypen des WBGUs (1999). Charakterisierung und Beispiele; $W=$ Eintrittswahrscheinlichkeit, $A=$ Schadensausmaß . 19

Tabelle 7: Neue Typen von Risiko-Managementstrategien nach Renn (2008). 20

\section{Abbildungen}

Abbildung 1: Maximal zulässige Deoxynivalenol (DON)-Gehalte von verzehrfertigem Getreide und DON-Aufnahme des durchschnittlichen Kleinkindes (2-4 Jahre) mit dem Getreidekonsum (insgesamt und je kg Körpergewicht) bei Ausschöpfung der zulässigen Grenzwerte laut VO (EG) Nr. 1881/2007 (durchgezogene Linie) im Vergleich zu den Werten bei der Einhaltung des TDI-Wertes (gestrichelte Linie). 


\section{Abkürzungen}

\begin{tabular}{|c|c|}
\hline Abkürzung & Bedeutung \\
\hline ALARA & As Low As Reasonably Achievable \\
\hline$B f R$ & Bundesinstitut für Risikoabschätzung \\
\hline BEE & Besondere Ernteermittlung \\
\hline BMELV & $\begin{array}{l}\text { Bundesministerium für Ernährung, Landwirtschaft und } \\
\text { Verbraucherschutz }\end{array}$ \\
\hline BSE & Bovine spongiforme Enzephalopathie \\
\hline DON & Deoxynivalenol \\
\hline FAEN & $\begin{array}{l}\text { Forschungsverbund Agrar- und Ernährungswissenschaften } \\
\text { Niedersachsen }\end{array}$ \\
\hline FAO & $\begin{array}{l}\text { Food and Agriculture Organization of the United Nations (Ernährungs- } \\
\text { und Landwirtschaftsorganisation der Vereinten Nationen) }\end{array}$ \\
\hline HT-2 & HT-2-Toxin \\
\hline IARC & International Agency for Research on Cancer \\
\hline NOAEL & No Observed Adverse Effect Level \\
\hline OECD & Organisation for Economic Co-operation and Development \\
\hline SCF & Scientific Committee on Food \\
\hline $\mathrm{T}-2$ & T-2-Toxin \\
\hline TDI-Wert & tolerable daily intake - Wert \\
\hline VELS-Studie & $\begin{array}{l}\text { Verzehrstudie zur Ermittlung der Lebensmittelaufnahme von Säuglingen } \\
\text { und Kleinkindern für die Abschätzung eines akuten Toxizitätsrisikos } \\
\text { durch Rückstände von Pflanzenschutzmitteln }\end{array}$ \\
\hline VO & Verordnung \\
\hline WBGU & $\begin{array}{l}\text { Wissenschaftlicher Beirat der Bundesregierung Globale } \\
\text { Umweltveränderungen }\end{array}$ \\
\hline WHO & World Health Organization \\
\hline
\end{tabular}




\section{Rahmen der Arbeit}

Die vorliegende Dissertation entstand im Rahmen des Forschungsverbundes „Qualitätsgerechte Pflanzenproduktion unter veränderten Rahmenbedingungen: Mykotoxine im Kontext von Produktion, Qualität und Verarbeitung“ (Forschungsverbund Agrar- und Ernährungswissenschaften Niedersachsen, FAEN 3) welcher durch das Niedersächsische Ministerium für Wissenschaft und Kultur gefördert wurde. Konkret wurde diese Dissertation während der Bearbeitung des Teilprojektes 8b „Implementierung eines kohärenten Managementsystems für Mykotoxinrisiken an Getreideprodukten in Niedersachsen: Effizienzanalyse" verfasst. Inhaltlich befasst sich die vorliegende Arbeit mit dem Gesundheitsrisiko durch das Fusarium-Mykotoxin Deoxynivalenol (DON) für Kinder, wobei der Schwerpunkt auf der Wahrnehmung dieses Risikos durch die Eltern und der Entwicklung und Evaluierung möglicher Verbesserungsansätze für das MykotoxinRisikomanagement liegt.

\section{Hintergrund der Arbeit}

Die Basis der vorliegenden Dissertation stellt die Arbeit von Raupach (2012) „Risiko und Risikomanagement ausgewählter Fusarium-Mykotoxine. Eine Analyse mit dem Fokus auf der Verbesserung des gesundheitlichen Verbraucherschutzes" dar, die in der ersten Projektphase des FAEN 3 entstanden ist. Zwei wesentliche Ergebnisse der Analyse von Raupach (2012) waren für diese Arbeit grundlegend. Zum einen die Feststellung, dass empfindliche Verbrauchergruppen, insbesondere Kleinkinder, durch die aktuellen Verbraucherschutzmaßnahmen nicht umfassend vor einer Gesundheitsgefährdung durch Mykotoxine, insbesondere durch DON, geschützt sind. Zum anderen die Klassifizierung des Mykotoxinrisikos anhand der Risikokategorien des Wissenschaftlichen Beirats der Bundesregierung zu Globalen Umweltveränderungen (WBGU, 1999) und darauf aufbauend die Ableitung möglicher Verbesserungsansätze für das Mykotoxin-Risikomanagement. Die von Raupach (2012) entwickelten allgemeinen Strategien zur Verbesserung des Gesundheitsschutzes vor Mykotoxinen wurden in dieser Arbeit konkretisiert, mit dem Ziel, einen umfassenden Verbraucherschutz zu gewährleisten, der empfindliche Verbrauchergruppen wie Kinder angemessen berücksichtigt. Die neu entwickelten 
Verbesserungsoptionen wurden in dieser Arbeit mittels einer Elternbefragung evaluiert und zudem ökonomisch bewertet.

\section{$2 \quad$ Einleitung}

Die gesundheitliche Unbedenklichkeit von Nahrungsmitteln ist in den letzten Jahren in Europa zu einem viel beachteten Thema geworden. Ausgelöst wurde das wachsende Interesse im Wesentlichen durch die BSE-Krise ${ }^{1}$ in den 1990er Jahren (Sinn, 2003). In Deutschland war das Thema "Lebensmittelsicherheit" seitdem immer wieder Gegenstand öffentlicher und politischer Diskussionen. Dabei standen häufig Lebensmittel tierischen Ursprungs im Mittelpunkt. Ein Beispiel aus der jüngsten Vergangenheit stellt der DioxinSkandal $^{2}$ im Jahr 2012 dar.

Auf Expertenseite wird verstärkt auch die Sicherheit pflanzlicher Lebensmittel kritisch hinterfragt (z. B. Raupach, 2012; Curtui et al., 2006; Banasiak et al., 2005). So kann die gesundheitliche Unbedenklichkeit getreidehaltiger Nahrungsmittel durch Kontaminanten natürlichen und unnatürlichen Ursprungs beeinträchtigt sein. $\mathrm{Zu}$ den unnatürlichen Schadstoffen zählen beispielsweise Pflanzenschutzmittelrückstände. Natürliche Verunreinigungen können durch natürlich vorkommende Schimmelpilzgifte, die Mykotoxine, verursacht werden. Nach Schätzungen der Food and Agriculture Organization of the United Nations (FAO) sind etwa 25\% aller Nahrungsmittel weltweit mit Mykotoxinen belastet ${ }^{3}$. Aktuell ist die Mykotoxinproblematik durch den Nachweis erhöhter Mengen des Aflatoxins $B_{1}$ in Futtermais in Deutschland, im Februar des Jahres 2013, stärker in die Öffentlichkeit gerückt. Die betroffenen Maispartien wurden an Kühe verfüttert, über deren Milch das Mykotoxin in die Lebensmittelkette gelangen kann. Mykotoxine werden aber auch regelmäßig in Getreideprodukten, die für die menschliche Ernährung bestimmt sind, nachgewiesen. Ein Beispiel für eine überhöhte Konzentration stellt der Rückruf

\footnotetext{
${ }^{1}$ Rinderkrankheit Bovine spongiforme Enzephalopathie (BSE), die auf den Menschen übertragbar ist und eine Form der Creutzfeldt-Jakob-Krankheit auslösen kann (BgVV, 2001).

${ }^{2}$ In Eiern unterschiedlicher deutscher Legehennenbetriebe wurden deutlich überhöhte Mengen des krebserregenden Dioxins nachgewiesen, URL: http://www.spiegel.de/wirtschaft/service/dioxin-behoerdenfinden-belastete-eier-aus-niedersachsen-a-827469.html und http://www.spiegel.de/wirtschaft/service/dioxinin-bio-eiern-gefunden-hof-in-nrw-gesperrt-a-825644.html.

${ }^{3}$ Weitere Stellungnahmen der FAO zur Mykotoxinproblematik sind unter URL: http://www.fao.org/docrep/007/y5499e/y5499e00.htm verfügbar.
} 
beziehungsweise der Verkaufsstopp biologisch erzeugter Nudeln, die Mykotoxine in unzulässigen Mengen enthielten, zu Beginn des Jahres 2011 dar ${ }^{4}$.

\section{Aufbau der Arbeit}

In dieser Arbeit wird zunächst auf die Entstehung von Mykotoxinen, ihr Potential zur Schädigung der menschlichen Gesundheit sowie auf die Eigenschaften und das Vorkommen der Mykotoxine eingegangen. Danach wird das staatliche Mykotoxin-Risikomanagement beschrieben. Anschließend werden Probleme der aktuellen Verbraucherschutzmaßnahmen aufgezeigt und es wird das Konzept der Risikowahrnehmung durch Laien und Experten erläutert. In Anlehnung an Raupach (2012) erfolgt dann eine wissenschaftliche Klassifizierung des Mykotoxinrisikos, bevor die von Raupach (2012) entwickelten Ansätze zur Verbesserung des Mykotoxin-Risikomanagements konkretisiert werden. Die potentiellen Verbesserungsmaßnahmen sollen insbesondere dazu dienen, das Gesundheitsrisiko durch Mykotoxine für empfindliche Verbrauchergruppen zu reduzieren. Es schließen sich fünf wissenschaftliche Beiträge an, welche im Rahmen dieser Dissertation entstanden sind. Der erste Beitrag widmet sich der Frage, ob eine Verbesserung des Gesundheitsschutzes vor Mykotoxinen aus ökonomischer Sicht sinnvoll ist. Der zweite Beitrag befasst sich grundlegend mit den Möglichkeiten der ökonomischen Bewertung von Kindergesundheit. Es folgt ein Artikel zu den elterlichen Regulierungspräferenzen hinsichtlich des Mykotoxinrisikos für ihre Kinder. Daran schließen sich zwei Beiträge an, die sich auf die elterliche Risikowahrnehmung von Mykotoxinen in Bezug auf die Gesundheit von Kindern und auf die elterliche Zahlungsbereitschaft für eine Verbesserung des Verbraucherschutzes beziehen. Abschließend werden ausgewählte Aspekte der oben genannten Beiträge diskutiert.

\section{Mykotoxine}

Mykotoxine sind natürlich vorkommende Schimmelpilzgifte, die die Lebensmittelsicherheit beeinträchtigen können. Nachstehend werden zunächst die Entstehung von Mykotoxinen

\footnotetext{
${ }^{4}$ Detailierte Informationen zu den Testergebnissen sind unter http://www.animal-healthonline.de/Ime/2011/03/24/nicht-verzehrsfahig-zwei-bio-nudelmarken-mit-schimmelpilzgift/5888/ abrufbar.
} 
und ihr Potential zur Gesundheitsschädigung beschrieben, bevor Vorkommen und Eigenschaften der Mykotoxine erläutert werden.

\subsection{Entstehung}

Mykotoxine sind niedermolekulare Stoffwechselprodukte bestimmter Pilze, mit unterschiedlicher Tier- und Humantoxität (Gareis, 1999; Bartels und Rodemann, 2003). Weltweit sind etwa 400 unterschiedliche Mykotoxine bekannt (Bartels und Rodemann, 2003). Davon sind jedoch nur etwa 20 Mykotoxine, aufgrund ihres Vorkommens und ihrer Konzentration in Nahrungs- und Futtermitteln, für den Menschen relevant (Gareis, 1999).

Mykotoxine werden von verschiedenen Feld- und Lagerpilzen gebildet. Die aus Sicht des gesundheitlichen Verbraucherschutzes bedeutsamen Mykotoxine werden hauptsächlich von fünf Pilzgattungen produziert. Dies sind im Einzelnen: Aspergillus, Penicillium, Fusarium, Alternaria und Claviceps (Gareis, 1999). In Tabelle 1 sind die für den Menschen wichtigsten Mykotoxine und die dazugehörigen Toxin-Produzenten dargestellt.

Tabelle 1: Die wichtigsten Mykotoxine in der menschliche Ernährung und ihre jeweiligen Produzenten.

\begin{tabular}{|l|l|}
\hline Pilzgattung (Mykotoxin-Bildner) & Wichtigste produzierte Mykotoxine \\
\hline Aspergillus & $\begin{array}{l}\text { Aflatoxin B1, G1, M1, Ochratoxin A, Sterigmatocystin, } \\
\text { Cyclopiazonsäure }\end{array}$ \\
\hline Penicillium & Ochratoxin A, Citrinin, Patulin, Cyclopiazonsäure, Penitrem A \\
\hline Fusarium & $\begin{array}{l}\text { Trichothecene (Deoxynivalenol, Nivalenol, , T-2 Toxin, HT-2 } \\
\text { Toxin, Diacetoxyscirpenol), Zearalenon, Fumonisine, } \\
\text { Moniliformin }\end{array}$ \\
\hline Alternaria & Tenuazonsäure, Alternariol, Alternariolmethylether \\
\hline Claviceps & Ergotalkaloide \\
\hline
\end{tabular}

Quelle: Gareis, 1999, S. 5.

Die Verbreitung der Pilze und damit die Bildung der Mykotoxine sind von unterschiedlichen Faktoren abhängig. Zum einen spielen die Anbaubedingungen und die Lagerhygiene eine wichtige Rolle (Bartels und Rodemann, 2003). Bedeutende Einflussfaktoren im Rahmen des Getreideanbaus sind Sortenwahl, Bodenbearbeitung, Vorfrucht und der Einsatz geeigneter Pflanzenschutzmittel (Bartels und Rodemann, 2003; Niens und Hasselmann, 2011). Zum 
anderen sind die Wetterbedingungen für eine Ausbreitung der Pilze entscheidend. Als günstig für das Pilzwachstum gilt im Allgemeinen ein feuchtwarmes Klima, wobei die Anforderungen der unterschiedlichen Pilzgattungen variieren (Bartels und Rodemann, 2003; Verreet und Aumann, 2002).

\subsection{Potential zur Schädigung der Gesundheit}

Die Aufnahme von Mykotoxinen über die Nahrung stellt für den Menschen ein Gesundheitsrisiko dar. Experten schätzen das Risiko durch Mykotoxine für den Menschen dabei als schwerwiegender ein als beispielsweise das Risiko durch bestimmungsgemäß angewendete Pflanzenschutzmittel. Kuiper-Goodman (2004, S. 4) äußert sich diesbezüglich wie folgt: „In terms of exposure and severity of chronic disease, especially cancer, mycotoxins appear at present to pose a higher risk than anthropogenic contaminants, pesticides (when used according to instructions), and food additives".

Allgemein werden Erkrankungen, welche durch Mykotoxine verursacht werden, als „Mykotoxikosen“ bezeichnet (Gareis, 1999). Mykotoxine können sowohl akut als auch chronisch toxisch wirken (Bennett und Klich, 2003). Das akute Gesundheitsrisiko durch Mykotoxine in Deutschland wird aufgrund guter Produktions- und Lagerbedingungen als gering eingeschätzt. Hingegen wird ein chronisches Gesundheitsrisiko infolge einer langfristigen Aufnahme kleiner Mykotoxinmengen derzeit auch für deutsche Verbraucher nicht ausgeschlossen (Raupach, 2012).

Da sich die einzelnen Mykotoxine stark hinsichtlich ihrer chemischen Struktur unterscheiden, ist auch ihre Wirkung auf die menschliche Gesundheit sehr unterschiedlich (Bartels und Rodemann, 2003). So können Mykotoxine unter anderem das Immunsystem beeinträchtigen (Aflatoxine, Deoxynivalenol, Ochratoxin A), mutagen wirken (Aflatoxine, Sterigmatocystin), Krebs erzeugen (Aflatoxine, Ochratoxin A, Fumonisine), die Haut und Zellen schädigen (Trichothecene) oder Nierenschäden hervorrufen (Ochratoxin A, Citrinin) (Gareis, 1999). Die auftretenden Gesundheitseffekte sind dabei nicht ausschließlich von dem aufgenommenen Mykotoxin abhängig. Weitere wichtige Faktoren wie die Aufnahmemenge und die Dauer der Exposition, aber auch Alter, Geschlecht, Gesundheitszustand und die Ernährungsgewohnheiten des Betroffenen beeinflussen die Art und Stärke der auftretenden Symptome. Zudem werden synergetische Wirkungen durch die gleichzeitige Aufnahme von 
Mykotoxinen mit anderen Toxinen vermutet, wobei genaue Wechselwirkungen bisher nur unzureichend bekannt sind (Bennett und Klich, 2003).

Problematisch ist weiterhin, dass viele Mykotoxine hinsichtlich ihrer Effekte auf die menschliche Gesundheit noch nicht vollständig erforscht sind (Raupach, 2012). Schwierigkeiten bereitet vor allem die differenzierte Analyse chronischer Gesundheitsfolgen (z.B. Krebserkrankungen, Immunsuppression), welche infolge einer langfristigen Aufnahme geringer Mykotoxinmengen auftreten können. So ist kaum eindeutig feststellbar, ob ein Mykotoxin ursächlich für den Ausbrauch der Krankheit war, beziehungsweise es ist nicht möglich, seinen Beitrag daran zu quantifizieren (Raupach, 2012). Klare Ursache-WirkungsZusammenhänge sind zudem aufgrund der Vielzahl weiterer Toxine und insbesondere karzinogener Stoffe, die die Umwelt belasten und zusätzlich zu Mykotoxinen aufgenommen werden, nicht eindeutig herstellbar (Reiß, 1976).

Die akuten Gesundheitsfolgen einer überhöhten Mykotoxinaufnahme sind hingegen besser erforscht, da die schnellen toxischen Reaktionen mit entsprechenden Symptomen die Feststellung eindeutiger Kausalitäten erlauben. Als Beispiele für akute und sehr schwerwiegende Vergiftungen, welche durch Mykotoxine ausgelöst wurden, führt Gareis (1999) zwei Fälle aus Russland und der Sowjetunion an: Ende des 9. Jahrhunderts traten in Russland zahlreiche Todesfälle auf, die ursächlich im Zusammenhang mit Brotgetreide standen, welches stark mit Pilzen der Gattung Fusarium und deren Toxinen belastet war. In der Sowjetunion starben tausende Menschen während des zweiten Weltkrieges an „Alimentärer Toxischer Aleukie“, nachdem sie stark mit Mykotoxinen kontaminiertes Getreide verzerrt hatten.

\subsection{Vorkommen und Eigenschaften}

Mykotoxine sind toxische, thermostabile und nicht als Allergen wirkende Naturstoffe, die im Rahmen des Sekundärstoffwechsels bestimmter Pilze gebildet werden (Gareis, 1999; Weidenbörner, 1999). Die Mykotoxine werden in Nahrungsmitteln auf der ganzen Welt nachgewiesen (Bennett und Klich, 2003). Häufig treten sie in Getreide einschließlich Mais und Nüssen auf, jedoch können auch Obst und Kaffeebohnen belastet sein. Eine völlige Vermeidung der Mykotoxine ist bislang nicht möglich (Wu, 2006). In tropischen und subtropischen Gegenden sind vor allem Pilze der Gattung Aspergillus, Penicillium und 
Alternaria verbreitet. Im Ackerbau der kühl-gemäßigten Breiten und damit auch in Deutschland dominieren hingegen die verschiedenen Fusarium-Arten und deren Toxine (Verreet und Aumann, 2002). Die einzelnen Fusarium-Arten können eine Vielzahl unterschiedlicher Mykotoxine bilden ${ }^{5}$. Deoxynivalenol (DON) stellt dabei das am häufigsten vorkommende Mykotoxin im Getreide dar (Bartels und Rodemann, 2003). Aufgrund seines häufigen Auftretens wird DON oft auch als Leittoxin der Fusarium-Mykotoxine bezeichnet (Dehne et al., 2002). Nach Büttner (2006) stellen Ährenfusariosen infolge ihrer starken Zunahme mittlerweile weltweit das größte Problem beim Anbau von Winterweizen dar.

Auch in Deutschland wird DON regelmäßig und in zum Teil deutlich überhöhten Konzentrationen in Weizen nachgewiesen (BMELV, 2012; Tabelle 2).

Tabelle 2: Deoxynivalenol (DON)-Gehalte in Weizen in Deutschland 2004-2012, angegeben in $\mu \mathrm{g} / \mathrm{kg}$ lufttrockenes Getreide.

\begin{tabular}{|l|l|l|l|l|}
\hline Jahr & Probenzahl & Mittelwert DON-Gehalt & Median DON-Gehalt & Min-Max DON-Gehalt \\
\hline 2004 & 505 & 268 & 69 & $<10-3528$ \\
\hline 2005 & 496 & 80 & 36 & $<10-4097$ \\
\hline 2006 & 471 & 88 & 16 & $<10-7543$ \\
\hline 2007 & 481 & 394 & 163 & $<10-12249$ \\
\hline 2008 & 486 & 70 & 16 & $<5-2506$ \\
\hline 2009 & 473 & 118 & 27 & $<5-7236$ \\
\hline 2010 & 458 & 127 & 27 & $<3-5005$ \\
\hline 2011 & 462 & 68 & 5 & $<3-2024$ \\
\hline 2012 & 473 & 367 & 120 & $<3-29.266$ \\
\hline
\end{tabular}

Quelle: BMELV, 2012, S. 43.

Aufgrund der herausragenden Bedeutung von Weizen für die Nahrungsmittelproduktion in Deutschland (BMELV, 2011) und aufgrund des häufigen Vorkommens von DON in diesem Getreide, konzentriert sich die vorliegende Arbeit ausschließlich auf Weizen und weizenhaltige Nahrungsmittel und mögliche Gesundheitsrisiken für den Menschen aufgrund einer Belastung mit dem Mykotoxin DON.

\footnotetext{
${ }^{5}$ Für eine zusammenfassende Darstellung siehe Raupach (2012, S. 10)
} 


\section{Deoxynivalenol (DON): Eigenschaften und Potential zur Schädigung der Gesundheit}

Da der Schwerpunkt der vorliegenden Arbeit auf dem Gesundheitsrisiko durch das Mykotoxin DON liegt, wird an dieser Stelle noch einmal detailliert auf das Schädigungspotential dieses Mykotoxins und seine spezifischen Eigenschaften eingegangen.

DON ist sehr stabil während der Lagerung und Vermahlung des Getreides (SCF, 1999). Dabei ist DON wasserlöslich (Curtui et al., 2006) und sehr hitzestabil (SCF, 1999). Daher wird es auch während des Backvorgangs nicht zerstört. Im verarbeiteten Endprodukt, wie beispielsweise in Brot, ist eine DON-Kontamination für den Verbraucher nicht mehr zu erkennen (Bartels und Rodemann, 2003).

DON kann sowohl akut als auch chronisch toxisch wirken. Als akute und subakute Effekte kann DON bei Aufnahme hoher Dosen Übergeben, Diarrhoe sowie Futterverweigerung und Gewichtsverluste bei landwirtschaftlichen Nutztieren auslösen (SCF, 1999; Bennett und Klich, 2003). Des Weiteren kann die Aufnahme von DON zum Absterben von Geweben und bei sehr großen Aufnahmemengen zum Tod führen (SCF, 1999). Zu den subchronischen Gesundheitseffekten, welche DON verursachen kann, zählen: reduzierte Futteraufnahme, reduzierte Gewichtszunahme bei Nutztieren sowie Veränderung verschiedener Blutparameter (SCF, 1999). Desweiteren kann DON die Fortpflanzung beeinflussen. Hinsichtlich einer karzinogenen Wirkung stuft die International Agency for Research on Cancer (IARC, 1993) DON aufgrund unzureichender Beweise als nicht klassifizierbar ein. In Bezug auf seine chronische Toxizität kann DON eine Schwächung des Immunsystems verursachen, infolge dessen sich die Anfälligkeit der Betroffen für Infektionskrankheiten erhöht (SCF, 1999). Insgesamt beurteilt das SCF (1999) DON insbesondere aufgrund seiner allgemeinen Toxizität und seiner Immuntoxizität als kritisch. Dabei ist jedoch anzumerken, dass DON hinsichtlich seiner Auswirkungen auf die menschliche Gesundheit noch nicht vollständig erforscht ist.

\section{Mykotoxin-Risikomanagement}

Der Begriff des „Risikomanagements“ bezeichnet entsprechend der VO (EG) Nr. 178/2002 der Europäischen Union den „Prozess der Abwägung strategischer Alternativen in Konsultation mit den Beteiligten unter Berücksichtigung der Risikobewertung und anderer 
berücksichtigenswerter Faktoren und gegebenenfalls der Wahl geeigneter Präventions- und Kontrollmöglichkeiten“. Laut WBGU (1999, S. 45) kann Risikomanagement auch als die „Summe der von Personen oder Organisationen eingeleiteten Maßnahmen zur Reduzierung, Steuerung und Regulierung von Risiken" angesehen werden. Risikomanagement soll entsprechend des Verständnisses des WBGUs (1999, S. 317) sicherstellen, „dass Systeme keine Zustände einnehmen, die nicht der gesellschaftlichen Risikopräferenz entsprechen“. Nachstehend werden die staatlichen Risikomanagement-Maßnahmen zum Schutz der Verbrauchergesundheit vor DON beschrieben. Desweiteren werden Probleme der aktuellen Regulierung aufgezeigt, welche aus Verbrauchersicht als kritisch anzusehen sind.

\subsection{Aktuelle Maßnahmen des gesundheitlichen Verbraucherschutzes vor Mykotoxinen}

Die Grundlage für den gesundheitlichen Verbraucherschutz vor Mykotoxinen und damit auch vor DON bilden die Verordnungen der Europäischen Union. Im Einzelnen sind insbesondere die VO (EWG) Nr. 315/93 des Rats der Europäischen Gemeinschaft, die Lebensmittelbasisverordnung VO (EG) Nr. 178/2002 sowie die VO (EG) Nr. 1881/2006 und die VO (EG) Nr. 1126/2007 des Rats der Europäischen Union relevant.

Die Basis des gesundheitlichen Verbraucherschutzes vor Mykotoxinen bildet die VO (EWG) Nr. 315/93, die besagt, dass kein Lebensmittel in Verkehr gebracht werden darf, „das einen Kontaminanten in einer gesundheitlich und insbesondere toxikologisch nicht vertretbaren Menge enthält".

Ein präventiver Gesundheitsschutz vor DON soll vorrangig durch die Festlegung von maximal zulässigen Höchstgehalten für Nahrungsmittel erreicht werden. Dabei dienen die zulässigen Höchstgehalte dazu, die öffentliche Gesundheit vor Beeinträchtigungen zu schützen (VO (EWG) Nr. 315/93).

Zudem müssen Grenzwerte für Mykotoxine entsprechend der VO (EG) Nr. 1881/2006 auf einem Niveau festgelegt werden, das durch gute Landwirtschafts- und Herstellungspraxis vernünftigerweise erreichbar ist („ALARA“: „as low as reasonable achievable“).

In der VO (EG) Nr. 1881/2006 beziehungsweise der VO (EG) Nr. 1126/2007 sind Grenzwerte für die maximale Belastung von Nahrungsmitteln mit DON festgelegt (Tabelle 3). Diese 
Grenzwerte sollen die Verbraucher davor schützen, mehr Mykotoxine aufzunehmen als gesundheitlich unbedenklich ist.

Tabelle 3: Maximal zulässige Höchstgehalte in $\mu \mathrm{g} / \mathrm{kg}$ Nahrungsmittel für Deoxynivalenol (DON) in Getreide und Getreideprodukten, die für den menschlichen Verzehr bestimmt sind.

\begin{tabular}{|l|c|}
\hline Nahrungsmittel & $\boldsymbol{\mu g}$ DON/kg Nahrungsmittel \\
\hline Unverarbeitetes Getreide außer Hartweizen, Hafer und Mais & 1250 \\
\hline Unverarbeiteter Hartweizen und Hafer & 1750 \\
\hline $\begin{array}{l}\text { Unverarbeiteter Mais außer unverarbeitetem Mais, der zur } \\
\text { Verarbeitung durch Nassmahlen bestimmt ist }\end{array}$ & 750 \\
\hline $\begin{array}{l}\text { Zum unmittelbaren menschlichen Verzehr bestimmtes Getreide, } \\
\text { Getreidemehl, als Enderzeugnis für den unmittelbaren menschlichen } \\
\text { Verzehr vermarktete Kleie und Keime }\end{array}$ & 750 \\
\hline Teigwaren (trocken) & 500 \\
\hline $\begin{array}{l}\text { Brot (einschließlich Kleingebäck), feine Backwaren, Kekse, Getreide- } \\
\text { Snacks und Frühstückscerealien }\end{array}$ & 200 \\
\hline Getreidebeikost und andere Beikost für Säuglinge und Kleinkinder & \\
\hline
\end{tabular}

Quelle: VO (EG) Nr. 1126/2007 (Auszug).

Die Aufnahmemenge DON, die aus toxikologischer Sicht als unbedenklich anzusehen ist, wird als Tolerable Daily Intake-Wert (TDI-Wert) bezeichnet. Konkret beschreibt der TDI-Wert die Menge DON, die täglich und lebenslang konsumiert werden kann, ohne dass eine Gesundheitsschädigung zu erwarten ist. Der TDI-Wert wird dabei in $\mu \mathrm{g}$ pro kg Körpergewicht angegeben. Das heißt, die Menge DON, die ein Mensch täglich aufnehmen kann, ohne in seiner Gesundheit beeinträchtigt zu werden, ist (auch) vom Körpergewicht des Betroffenen abhängig. Der Festlegung des TDI-Wertes für DON erfolgte durch das Scientific Committee on Food und beruht auf toxikologischen Studien an Nagetieren. Für DON wurde ein TDI von 1 g/kg Körpergewicht (SCF, 1999; 2002) ermittelt. Zur Übertragbarkeit des Wertes auf den Menschen wird die Menge DON, bei der in Langzeitstudien keine negativen Gesundheitseffekte („No Observed Adverse Effects Levels“ (NOAEL)) beobachtet werden konnten unter Verwendung eines Sicherheitsfaktors von 100 modifiziert. Dieser Sicherheitsfaktor ergibt sich einerseits aus Unsicherheiten bei der Übertragung des Wertes von Tieren auf den Menschen (Pieters et al., 2002). Andererseits soll damit individuellen Unterschieden in der Empfindlichkeit unterschiedlicher Verbrauchergruppen (z. B. von Kindern) Rechnung getragen werden (Pieters et al., 2002). 


\subsection{Defizite der aktuellen Verbraucherschutzmaßnahmen und Risiken für den}

\section{Verbraucher}

Aus Verbrauchersicht kritisch zu bewerten ist, dass auch bei Einhaltung der derzeit gültigen DON-Grenzwerte nicht ausgeschlossen werden kann, dass bestimmte Konsumentengruppen mehr DON aufnehmen, als gesundheitlich unbedenklich ist. Das heißt, die derzeitigen Risikomanagement-Maßnahmen gewährleisten keinen vollständigen Gesundheitsschutz aller Verbraucher. Diesbezüglich zeigt Raupach (2012) auf Basis einer Berechnung der möglichen DON-Aufnahme im Rahmen der zulässigen Höchstgehalte gemäß der VO (EG) Nr. 1881/2006 und VO (EG) Nr. 1881/2007, dass Kinder zwischen zwei und vier Jahren den TDI, also die gesundheitlich unbedenkliche Menge DON, um fast das Vierfache überschreiten können. Hierfür betrachtet Raupach (2012, 115 ff.) Verzehrdaten zwei- bis vierjähriger Kinder, die im Rahmen der VELS-Studie ermittelt wurden (Verzehrstudie zur Ermittlung der Lebensmittelaufnahme von Säuglingen und Kleinkindern für die Abschätzung eines akuten Toxizitätsrisikos durch Rückstände von Pflanzenschutzmitteln. BfR, 2005; Banasiak et al., 2005). Gemäß dieser Studie entspricht die durchschnittliche Getreideverzehrmenge eines zwei- bis vierjährigen Kindes 85,6 g pro Tag bei einen durchschnittlichen Körpergewicht von 16,15 kg. Der TDI-Wert für DON beträgt $1 \mu \mathrm{g}$ pro kg Körpergewicht und Tag. Laut VO (EG) Nr. 1881/2006 darf ein Kilo Getreide, das unmittelbar für den menschlichen Verzehr vorgesehen ist, maximal $750 \mu \mathrm{g}$ DON enthalten. Hieraus folgt, dass $85,6 \mathrm{~g}$ verzehrfertiges Getreide bis zu $64,2 \mu \mathrm{g}$ DON beinhalten darf. Wird diese Menge DON in das Verhältnis zum durchschnittlichen Körpergewicht von $16,15 \mathrm{~kg}$ gesetzt, zeigt sich, dass Kleinkinder bei Ausschöpfung der aktuellen Grenzwerte 3,98 $\mu \mathrm{g}$ DON pro Kilo Körpergewicht aufnehmen. In diesem Fall würde die gesundheitliche unbedenkliche Aufnahmemenge von $1 \mu \mathrm{g}$ DON pro Kilo Körpergewicht also deutlich überschritten. Um eine TDI-Überschreitung durchschnittlicher Kleinkinder zu vermeiden dürften in einem Kilo verzehrfertigem Getreide maximal $189 \mu \mathrm{g}$ DON, anstelle der derzeit erlaubten $750 \mu \mathrm{g}$ DON, enthalten sein (Raupach, 2012, S. 116).

Abbildung 1 veranschaulicht die oben dargestellte Betrachtung. Dabei zeigt der linke, grüne Teil der Pfeile die Werte, bei der es zu keiner TDI-Wert Überschreitung kommt. Die Werte, bei der eine TDI-Wert Überschreitung vorliegt, liegen im mittleren, gelben Teil. Dieser Bereich spiegelt die aktuelle Situation hinsichtlich des gesundheitlichen Verbraucherschutzes 
vor DON wieder, bezogen auf ein durchschnittliches Kleinkind im Alter zwischen zwei und vier Jahren.

Abbildung 1: Maximal zulässige Deoxynivalenol (DON)-Gehalte von verzehrfertigem Getreide und DON-Aufnahme des durchschnittlichen Kleinkindes (2-4 Jahre) mit dem Getreidekonsum (insgesamt und je kg Körpergewicht) bei Ausschöpfung der zulässigen Grenzwerte laut VO (EG) Nr. 1881/2007 (durchgezogene Linie) im Vergleich zu den Werten bei der Einhaltung des TDI-Wertes (gestrichelte Linie).
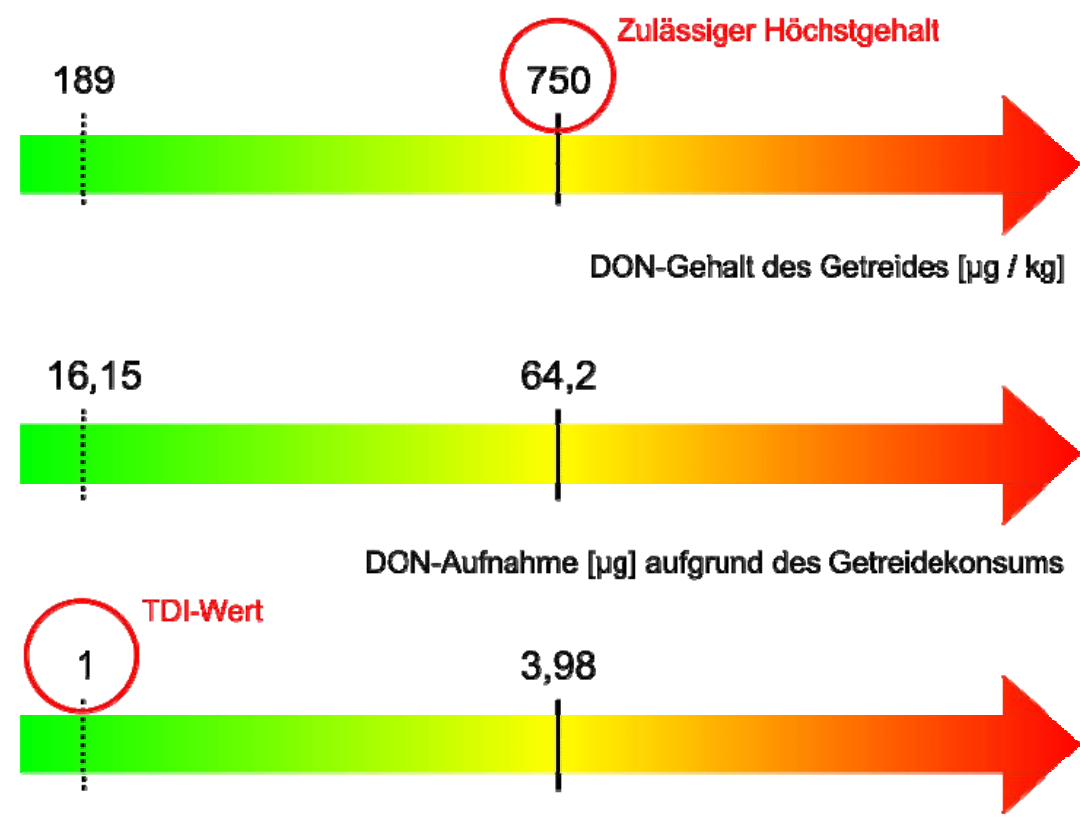

DON-Aufnahme [ug] je kg Körpergewicht mit dem Getreidekonsum

Quelle: Raupach, 2012, S. 116.

Hinsichtlich der Betrachtung von Raupach (2012, S. 115ff.) ist jedoch zu beachten, dass die dargestellten Berechnungen auf Durchschnittswerten beruhen. Bei Kindern, die zu GetreideVielverzehren zählen, ist demnach eine weitaus deutlichere TDI-Überschreitung möglich. Andererseits führt eine freiwillige Unterschreitung der maximal zulässigen DONHöchstgehalte durch die Lebensmittelunternehmer zu einer Minderung der tatsächlichen täglichen DON-Aufnahmemenge (Raupach, 2006, S. 116). In Jahren mit hohem Fusariumdruck und entsprechend hohen DON-Belastungen des Getreides kann allerdings nicht ausgeschlossen werden, dass TDI-Überschreitungen bei Kleinkindern auch tatsächlich vorkommen. Im Jahr 2007 muss beispielsweise davon ausgegangen werden, dass das durchschnittliche Kleinkind den TDI von $1 \mu \mathrm{g}$ pro kg Körpergewicht und Tag um etwa 25\% überschritten hat (Raupach und Marggraf, 2012). 
Neben Raupach (2012) und Raupach und Marggraf (2012) weisen auch andere Autoren auf Lücken des derzeitigen DON-Risikomanagements hin. Curtui et al. (2006, S. 109 ff.) zeigen, ähnlich wie Raupach (2012), dass es bei Kindern, aber auch bei Jugendlichen und jungen Erwachsenen bis zu einem Alter von 24 Jahren zu Überschreitungen des TDI-Wertes kommen kann. In Abhängigkeit von der Verzehrmenge und der zugrundegelegten DONBelastung der konsumierten Lebensmittel ist bei Kindern zwischen vier und sechs Jahren eine durchschnittliche Überschreitung des TDIs um das 2,7-fache möglich. Kinder zwischen sieben und neuen Jahren könnten durchschnittlich etwa doppelt so viel DON aufnehmen wie aus toxikologischer Sicht unbedenklich ist. Aber auch Erwachsene bis zum vierundzwanzigsten Lebensjahr schöpfen den TDI entsprechend der Betrachtung von Curtui et al. (2006) bis zu $110 \%$ aus.

Vor dem Hintergrund der oben beschriebenen möglichen TDI-Überschreitungen, insbesondere von Kindern, müssen die aktuellen Verbraucherschutzmaßnahmen vor DON aus Konsumentensicht als nicht zufriedenstellend beurteilt werden. Es stellt sich die Frage, wie die Verbraucher das Gesundheitsrisiko durch Mykotoxine für Kinder und die aktuellen Verbraucherschutzmaßnahmen beurteilen.

\subsection{Mykotoxin-Risikowahrnehmung aus Konsumentensicht}

In Bezug auf die Bewertung von Risiken besteht häufig eine Diskrepanz zwischen der Risikowahrnehmung von Laien und der Risikowahrnehmung von Experten (Raupach, 2012, Raupach und Marggraf, 2011; Slovic, 1987). Slovic (1987) führt diese Diskrepanz auf unterschiedliche Definitionen des Konstrukts „Risiko“ zurück. Dabei wird die Risikowahrnehmung von Experten oft als „objektiv“ bezeichnet, weil sie auf Fakten wie der jährlichen Todesrate (Slovic, 1987, Slovic et al. 2000a) oder dem Schadensausmaß und der Realisierungswahrscheinlichkeit einer Gefahr beruht (WBGU, 1999). Die Risikowahrnehmung von Laien kann hingegen eher als intuitiv oder auch subjektiv charakterisiert werden. Renn (2005) unterscheidet dabei zwei Klassen qualitativer Wahrnehmungsmuster: 
- Risikobezogene Muster: Schrecklichkeit der Folgen, Gewöhnung, sinnliche Wahrnehmbarkeit, Natürlichkeit

- Situationsbezogene Muster: Freiwilligkeit, persönliche Kontrollierbarkeit, gerechte Nutzen- und Risikoverteilung, Vertrauen in das Risikomanagement

Die Darstellung der risikobezogenen Muster nach Renn (2005) verdeutlicht, dass die Bewertung eines Risikos durch Laien von subjektiv wahrgenommen Merkmalen der betrachteten Gefahr und situativen Hintergrundvariablen bestimmt wird. Im Vergleich zur Risikowahrnehmung von Experten beruht sie deutlich stärker auf Konstruktionsprozessen, weil Faktenwissen über die betrachtete Gefahr in der Regel nicht vorhanden ist.

Hinsichtlich der Wahrnehmung und Bewertung des Gesundheitsrisikos durch Mykotoxine muss die Gesamtgruppe der Konsumenten als Laien angesehen werden. Nach Hansen et al. (2003) kann die Wahrnehmung eines Risikos durch Laien als ein komplexer, situations- und gefühlsbezogener Ausdruck des Wertesystems einer Person aufgefasst werden. Folgt man diesem Verständnis von Risikowahrnehmung, erklärt sich, warum unterschiedliche Personen dasselbe Risiko verschieden bewerten.

Zur Messung der Wahrnehmung eines Risikos aus Laiensicht hat sich das „Psychometrische Paradigma" durchgesetzt, das von Paul Slovic und seinen Mitarbeitern (1987, 2000, 2000a) entwickelt wurde. Der Ansatz von Slovic (1987) und Slovic et al. (2000, 2000a) besagt, dass die öffentliche Wahrnehmung eines Risiko jeweils durch die spezifischen Eigenschaften der betrachten Gefahr beschrieben werden kann. Jedes Risiko besitzt entsprechend ein Muster an Charakteristika, das über seine öffentliche Wahrnehmung bestimmt. Die unterschiedlichen, subjektiv wahrgenommenen Eigenschaften der betrachteten Risiken lassen sich in der Regel zu drei Faktoren zusammenfassen (Tabelle 4).

Den größten Einfluss auf die Laien-Wahrnehmung eines Risikos hat der Faktor "Dread" (Slovic, 1987). Je mehr Angst ein Risiko hervorruft, desto schwerwiegender wird es empfunden. Entsprechend steigt mit dem Potential eines Risikos zur Angsterzeugung auch die Anzahl der Personen, die eine Risikoreduzierung wünschen und eine strengere Regulierung befürworten (Slovic, 1987). 
Tabelle 4: Risikocharakteristika, die die Laien-Wahrnehmung eines Risikos beschreiben, zusammengefasst zu drei Faktoren.

\begin{tabular}{|l|l|l|}
\hline & Faktor & Risikocharakteristika \\
\hline 1. & $\begin{array}{l}\text { Das Potential des Risikos zur } \\
\text { Angsterzeugung (Dread) }\end{array}$ & $\begin{array}{l}\text { unkontrollierbares Schadensausmaß (severity not } \\
\text { controllable), angsterzeugend (dread), katastrophal } \\
\text { (catastrophic), schwierig zu verhindern (hard to prevent), } \\
\text { tödlich (fatal), ungerecht (inequitable), relevant für } \\
\text { zukünftige Generationen (threatening for future } \\
\text { generations), nicht leicht reduzierbar (not easily reduced), } \\
\text { zunehmend (increasing), unfreiwillig (involuntary), } \\
\text { persönlich betreffend (threatening to the rather } \\
\text { personally) }\end{array}$ \\
\hline 2. & $\begin{array}{l}\text { Die Vertrautheit des Risikos } \\
\text { (Familiarity) }\end{array}$ & $\begin{array}{l}\text { Unmittelbarkeit der Konsequenzen (immediacy of } \\
\text { consequences), Vertrautheit (familiarity) }\end{array}$ \\
\hline 3. & $\begin{array}{l}\text { Die Anzahl der durch das Risiko } \\
\text { betroffene Personen (People } \\
\text { exposed) }\end{array}$ & $\begin{array}{l}\text { Anzahl der dem Risiko ausgesetzten Personen (many } \\
\text { people exposed) }\end{array}$ \\
\hline
\end{tabular}

Quelle: Slovic et al., 2000, S. 141.

Der Psychometrische Ansatz von Slovic (1987) wurde von Fife-Schaw und Rowe (2000) weiterentwickelt und dem speziellen Anwendungsfeld der Lebensmittelrisiken angepasst. Es resultierte der Perceived Food Risk Index (PFRI), eine Skala, welche insgesamt zehn Items umfasst und der Messung der öffentlichen Wahrnehmung von Lebensmittelrisiken dient. Der PFRI wurde im Rahmen dieser Arbeit auf das Themengebiet "Mykotoxine und Kindergesundheit" angewendet, um die Laien-Wahrnehmung des Mykotoxin (DON)-Risikos zu untersuchen. Da Kinder als direkt Betroffene aufgrund ihres kognitiven Entwicklungsstandes nicht direkt nach ihrer Einschätzung des Gesundheitsrisikos durch DON befragt werden können, muss die Erhebung der Risikowahrnehmung über Stellvertreter erfolgen. Entsprechend der Working Party on National Environmental Policy (2005) sind Eltern durch ihre emotionalen Nähe und ihre Verantwortlichkeit für die Gesundheit ihres Kindes am besten in der Lage, die Einstellungen und Präferenzen ihrer Kinder wiederzugeben $^{6}$. Daher sollte die Messung über die Eltern erfolgen. Folglich wurde im Rahmen dieser Arbeit die elterliche Risikowahrnehmung von Mykotoxinen (DON) in Getreideprodukten in Bezug auf die Gesundheit von Kindern in Niedersachsen erfasst ${ }^{7}$.

\footnotetext{
${ }^{6}$ Siehe hierzu ausführlich Niens und Marggraf, (2010).

${ }^{7}$ Im Rahmen der Elternbefragung wurde allgemein von Mykotoxinen (Schimmelpilzgiften) gesprochen, um eine Überforderung der Befragten durch weitere Konkretisierung und neue Begrifflichkeiten zu vermeiden. Konkret
} 


\section{Einflussfaktoren der Beurteilung der Schwere des Risikos und der Risikoakzeptanz}

Bei der Beurteilung eines Risikos durch Laien konnten bestimmte Zusammenhänge zwischen den subjektiv wahrgenommenen Merkmalen eines Risikos und der Beurteilung der Schwere der Gefahr beziehungsweise ihrer Akzeptanz beobachtet werden. Starr (1969) und Fischhoff et al. (2000) stellten diesbezüglich fest, dass Risiken, die einen großen Nutzen stiften, eher von der Gesellschaft als akzeptabel angesehen werden. Gleiches gilt für freiwillig eingegangene Risiken wie Ski- oder Autofahren. Auch diese werden deutlich eher toleriert als unfreiwillige Gesundheitsgefahren, welche beispielsweise durch Kontaminationen von Lebensmitteln verursacht werden können (Starr, 1969; Fischhoff et al. 2000). Desweiteren werden natürliche Risiken als weniger schwerwiegend eingestuft als unnatürliche Risiken (Fife-Schaw und Rowe, 1996; Renn 2005). Auch die Zeitspanne zwischen Handlung und dem Eintritt eines Schadens wirken sich auf die Wahrnehmung eines Risikos aus. Je unmittelbarer sich ein Schaden realisiert, desto höher wird ein Risiko eingeschätzt (WBGU, 1999, S. 178) und desto weniger wird es von der Gesellschaft akzeptiert (Fischhoff et al. 2000).

Für die Bewertung eines Risikos sind außerdem die Eigenschaften eines Individuums entscheidend. Hier sind vor allem die Einstellung und das Verhältnis zum Risikoverursacher, das Vertrauen in die für eine Risikoregulierung zuständige Behörde und die Lebenssituation des Betroffenen (Alter, ökonomische Situation, Vorhandensein von Kindern) zu nennen (WBGU, 1999, S. 171ff.). Aber auch schlechte persönliche Erfahrungen mit einer identischen oder einer ähnlichen wie der zu beurteilenden Gefahr können zu einer hohen Risikowahrnehmung führen (WBGU, 1999, S.178).

Es ist davon auszugehen, dass die oben aufgeführten Risikoeigenschaften gemeinsam mit den persönlichen Merkmalen der betroffenen Personen auch die elterliche Risikowahrnehmung von Mykotoxinen beeinflussen. Sie bestimmen einerseits darüber, wie schwerwiegend das Mykotoxinrisiko durch die Eltern eingestuft wird. Andererseits haben sie vermutlich einen Einfluss auf die Akzeptanz des Risikos durch die befragten Eltern. 


\subsection{Grundlagen der wissenschaftlichen Klassifizierung von Risiken entsprechend des}

\section{WBGUs}

Vom dem Begriff der intuitiven Risikowahrnehmung durch Laien, der in Kapitel 4.3 beschrieben wurde, muss die wissenschaftliche Risikoanalyse und die Risikoklassifizierung unterschieden werden. Die Risikoanalyse wird von Experten vorgenommen und vielfach als rational bezeichnet, weil sie sich im Wesentlichen auf Sach-, Gesundheits- und Umweltschäden beschränkt (WBGU, 1999, S. 40). Damit bildet die Risikoanalyse die Basis für eine wissenschaftliche Klassifizierung eines Risikos (WBGU, 1999). Laut WBGU (1999, S. 308) lässt sich der Begriff „Risiko“ rational folgendermaßen pauschal charakterisieren:

$$
\mathbf{R}=\mathbf{W} \cdot \mathbf{A} \text {, }
$$

wobei $W\{0,1\}$ die Eintrittswahrscheinlichkeit des schadenswirksamen Ereignisses und $A>0$ das Schadensausmaß bezeichnet. Damit stellen die Eintrittswahrscheinlichkeit und das Schadensausmaß die beiden zentralen Kategorien des wissenschaftlichen Risikokonzepts laut WBGU (1999) dar. Wichtig in diesem Zusammenhang ist zudem die Abschätzungssicherheit dieser beiden Größen. Der Begriff der Abschätzungssicherheit bezeichnet den Grad der Verlässlichkeit, mit der eine Aussage über die Wahrscheinlichkeit von Schadensereignissen getroffen werden kann (WBGU, 1999, S. 53).

Das Schadensausmaß und die Eintrittswahrscheinlichkeit sowie deren Abschätzungssicherheit sind nach Auffassung des WBGUs (1999) für die wissenschaftliche Einordnung eines Risikos allein nicht ausreichend. Vor diesem Hintergrund hat der WBGU (1999, S. 55f.) weitere Kriterien für die Klassifizierung eines Risikos vorgeschlagen, die in einigen Ländern (Dänemark, Schweiz, Niederlande) bereits berücksichtigt werden. Diese zusätzlichen Kriterien sind in Tabelle 5 dargestellt. 
Tabelle 5: Wichtige Kriterien für die wissenschaftliche Klassifizierung von Risiken entsprechend des WBGUs (1999).

\begin{tabular}{|c|c|}
\hline $\begin{array}{l}\text { Kriterium der } \\
\text { Risikoklassifizierung }\end{array}$ & Beschreibung \\
\hline Ubiquität & $\begin{array}{l}\text { Räumliche Verbreitung des Schadens oder des Schadenspotentials } \\
\text { (intragenerationale Gerechtigkeit) }\end{array}$ \\
\hline Persistenz & $\begin{array}{l}\text { Zeitliche Ausdehnung des Schadens oder des Schadenspotentials } \\
\text { (intergenerationale Gerechtigkeit) }\end{array}$ \\
\hline Irreversibilität & $\begin{array}{l}\text { Nichtwiederherstellbarkeit des Zustandes vor Schadenseintritt. } \\
\text { Dabei geht es im Umweltbereich vorrangig um die typenmäßige } \\
\text { Wiederherstellbarkeit im Rahmen eines dynamischen Wandels (etwa } \\
\text { Wiederaufforstung oder Reinigung des Wassers), nicht um die } \\
\text { individuelle Restaurierung des Urzustandes (etwa Erhalt eines } \\
\text { individuellen Baumes oder Ausmerzung nicht einheimischer } \\
\text { Pflanzen- und Tierarten) }\end{array}$ \\
\hline Verzögerungswirkung & $\begin{array}{l}\text { Damit ist die Möglichkeit gemeint, das zwischen dem auslösenden } \\
\text { Ereignis und der Schadensfolge eine lange Latenzzeit herrscht. Diese } \\
\text { Latenzzeit kann physikalischer (langsame Reaktionsgeschwindigkeit), } \\
\text { chemischer oder biologischer Natur sein (etwa bei vielen } \\
\text { Krebserkrankungen oder mutagenen Veränderungen) oder sich als } \\
\text { Folge einer langen Variablenkette (etwa Aussetzen des Golfstroms } \\
\text { aufgrund von Klimaveränderungen) ergeben }\end{array}$ \\
\hline $\begin{array}{l}\text { Mobilisierungspotential } \\
\text { (Akzeptanzverweigerung) }\end{array}$ & $\begin{array}{l}\text { Darunter versteht man die Verletzung von individuellen, sozialen } \\
\text { oder kulturellen Interessen und Werten, die eine entsprechende } \\
\text { Reaktion der Betroffenen hervorruft. Diese Reaktionen umfassen } \\
\text { den offenen Protest, den Entzug von Vertrauen in die } \\
\text { Entscheidungsträger, geheime Sabotageakte oder andere Formen } \\
\text { der Gegenwehr. Auch psychosomatische Folgen lassen sich in diese } \\
\text { Kategorie aufnehmen. }\end{array}$ \\
\hline
\end{tabular}

Quelle: WBGU, 1999, S. 55.

Unter Verwendung der in Tabelle 5 beschriebenen Risikocharakteristika und der zentralen Kategorien Eintrittswahrscheinlichkeit und Schadensausmaß hat der WBGU (1999, S. 58ff.) sechs unterschiedliche Risikotypen entwickelt. Diese sind in Tabelle 6 überblickartig zusammengefasst. Die Zuordnung eines Risikos zu einem der Risikotypen erfolgt entsprechend seiner Ausprägungen der durch den WBGU (1999) benannten Risikocharakteristika, insbesondere der Eintrittswahrscheinlichkeit, des Schadensausmaßes und der Abschätzungssicherheit dieser zentralen Kriterien der Risikocharakteristika.

Die Risikoklassifizierung dient dem Zweck, Risiken gruppenweise beurteilen und managen zu können. In diesem Zusammenhang hat Renn (2008, S. 291) grundlegende Managementstrategien für jeden der dargestellten Risikotypen vorgeschlagen (Tabelle 7). 
Tabelle 6: Überblick über die Risikotypen des WBGUs (1999). Charakterisierung und Beispiele; $\mathbf{W}=$ Eintrittswahrscheinlichkeit, $A=S$ chadensausmaß.

\begin{tabular}{|c|c|c|}
\hline Risikotyp & Charakterisierung & Beispiele \\
\hline Damokles & $\begin{array}{l}\text { - } \quad \text { W gering (gegen 0) } \\
\text { - } \text { Abschätzungssicherheit von W hoch } \\
\text { - } \quad \text { A hoch (gegen unendlich) } \\
\text { - }\end{array}$ & $\begin{array}{l}\text { - Kernenergie } \\
\text { - } \text { Großchemische Anlagen } \\
\text { - } \text { Staudämme } \\
\text { - } \text { Meteoriteneinschläge }\end{array}$ \\
\hline Zyklop & $\begin{array}{l}\text { - W ungewiss } \\
\text { - Abschätzungssicherheit von W } \\
\text { ungewiss } \\
\text { - A hoch } \\
\text { - Abschätzungssicherheit von A eher } \\
\text { hoch }\end{array}$ & $\begin{array}{l}\text { - Erdbeben } \\
\text { - } \text { Vulkaneruptionen } \\
\text { - } \text { AIDS-Infektion } \\
\text { - Massenentwicklungen anthropogen } \\
\text { beeinflusster Arten } \\
\text { - Zusammenbruch der thermohalinen } \\
\text { Zirkulation }\end{array}$ \\
\hline Pythia & $\begin{array}{l}\text { - } \text { W ungewiss } \\
\text { - Abschätzungssicherheit von W } \\
\text { ungewiss } \\
\text { - A ungewiss (potentiell hoch) } \\
\text { - Abschätzungssicherheit von A } \\
\text { ungewiss }\end{array}$ & $\begin{array}{l}\text { - Sich aufschaukelnder Treibhauseffekt } \\
\text { - } \text { Freisetzung und Inverkehrbringen } \\
\text { transgener Pflanzen } \\
\text { - BSE/nv-CJD-Infektion } \\
\text { - Bestimmte Anwendungen der } \\
\text { Gentechnologie } \\
\text { - Instabilität der westantarktischen } \\
\text { Eisschilde }\end{array}$ \\
\hline Pandora & $\begin{array}{l}\text { - } \text { W ungewiss } \\
\text { - Abschätzungssicherheit von W } \\
\text { ungewiss } \\
\text { - A ungewiss (nur Vermutungen) } \\
\text { Abschätzungssicherheit von A } \\
\text { ungewiss } \\
\text { - Persistenz hoch (mehrere } \\
\text { Generationen) }\end{array}$ & $\begin{array}{l}\text { - Persistente organische Schadstoffe } \\
\text { (POP) } \\
\text { - Endokrin wirksame Stoffe }\end{array}$ \\
\hline Kassandra & $\begin{array}{l}\text { - W eher hoch } \\
\text { - } \text { Abschätzungssicherheit von W eher } \\
\text { - } \text { Aering } \\
\text { - Abschë hoch } \\
\text { - hoch } \\
\text { - Verzögerungswirkung hoch }\end{array}$ & $\begin{array}{l}\text { - Anthropogener schleichender } \\
\text { Klimawandel } \\
\text { - Destabilisierung terrestrischer } \\
\text { Ökosysteme }\end{array}$ \\
\hline Medusa & $\begin{array}{l}\text { - W eher gering } \\
\text { - Abschätzungssicherheit von W eher } \\
\text { gering } \\
\text { - A eher gering (Exposition hoch) } \\
\text { - Abschätzungssicherheit von A eher } \\
\text { - } \text { hoch } \\
\text { - Mobilisierungspotential hoch }\end{array}$ & - Elektromagnetische Felder \\
\hline
\end{tabular}

Quelle: WBGU, 1999, S. 161. 
Tabelle 7: Neue Typen von Risiko-Managementstrategien nach Renn (2008).

\begin{tabular}{|c|c|c|c|c|}
\hline Management & Risikotyp & Schaden & Wahrscheinlichkeit & Handlungsstrategien \\
\hline \multirow[t]{2}{*}{ Risikoorientiert } & Damokles & hoch & gering & \multirow{2}{*}{$\begin{array}{ll}\text { - } & \text { Katastrophenpotential } \\
\text { - } & \text { Wahruzieren } \\
\text { ermitteln } \\
\text { - } \\
\text { Robustere Lösungen } \\
\text { anstreben } \\
\text { - Überraschungen } \\
\text { verhindern } \\
\text { - Katastrophenmanagement }\end{array}$} \\
\hline & Zyklop & hoch & ungewiss & \\
\hline \multirow[t]{2}{*}{ Vorsorgeorientiert } & Pythia & ungewiss & ungewiss & \multirow{2}{*}{$\begin{array}{ll}\text { - } & \text { Vorsorgeprinzip } \\
\text { - } & \text { implementieren } \\
\text { - } & \text { Intensibstitute entwickeln } \\
\text { - } & \text { Resilienz verbessern } \\
\text { - } & \text { Containment (Ort, Zeit) }\end{array}$} \\
\hline & Pandora & ungewiss & ungewiss & \\
\hline \multirow[t]{2}{*}{ Diskursiv } & Kassandra & hoch & hoch & \multirow{2}{*}{$\begin{array}{ll}\text { - } & \text { Bewusstseinsbildung } \\
\text { - } & \text { Vertrauensbildung } \\
\text { - } & \text { Organisatorisches Lernen } \\
\text { - } & \text { Wissen verbessern } \\
\text { - } & \text { Sicherheitskultur } \\
& \text { verbessern }\end{array}$} \\
\hline & Medusa & gering & gering & \\
\hline
\end{tabular}

Quelle: Renn, 2008, S. 291.

Laut WBGU (1999, S .63) stellt die hier beschriebene Risikotypologie eine Art „Merkliste“ für Risikoexperten und für Risikolaien dar, um eine der Bedrohung angemessene Risikoerfassung und -bewertung vorzunehmen zu können. Insofern dienen die Risikoklassifizierung und die dazugehörigen allgemeinen Risikomanagementstrategien der Vereinfachung des Umgangs mit Risiken.

\subsubsection{Einordnung des DON-Risikos in die Risikotypologie des WBGUs}

Das Gesundheitsrisiko durch DON zeichnet sich durch seine unsichere Eintrittswahrscheinlichkeit, ein ungewisses, aber potentiell hohes, Schadensausmaß und hohe Ubiquität aus. Die Abschätzungssicherheit von Eintrittswahrscheinlichkeit und Schadensausmaß sind ungewiss. Das Mobilisierungspotential des Risikos durch DON ist als gering einzuschätzen. Hinsichtlich der Verzögerungswirkung können nach derzeitigem Kenntnisstand keine Aussagen getroffen werden. Die Persistenz und die Reversibilität des 
durch DON verursachten Schadens sind ebenfalls ungewiss (Raupach, 2012, S. 77). Damit muss das Gesundheitsrisiko durch DON dem Risikotyp „Pythia“ zugeordnet werden ${ }^{8}$.

\subsubsection{Ableitung geeigneter Verbesserungsansätze für das DON-Risikomanagement zur} Verbesserung des Gesundheitsschutzes von Kindern

Für das Management von Risiken des Typs „Pythia“ werden von Renn (2008, S. 291) vorsorgeorientierte Handlungsstrategien vorgeschlagen (vgl. Tabelle 7). Durch die Festlegung von Grenzwerten für die maximal zulässige Belastung von Nahrungsmitteln mit DON gemäß der VO(EG) Nr. 1881/2006 und der VO(EG) Nr. 1126/2007 sowie die Durchführung regelmäßiger Lebensmittelkontrollen wird diesem Prinzip bereits Rechnung getragen ${ }^{9}$. Jedoch erscheint eine Verbesserung des derzeitigen Mykotoxin-Risikomanagements vor dem Hintergrund, dass Kinder auch bei Einhaltung der gültigen DON-Grenzwerte mehr DON aufnehmen könnten als gesundheitlich unbedenklich ist, wünschenswert.

Für eine Verbesserung des Gesundheitsschutzes von Kindern vor DON käme eine Intensivierung des Vorsorgeprinzips beispielsweise durch die Senkung der aktuellen DONGrenzwerte von $750 \mu \mathrm{g} / \mathrm{kg}$ verzehrfertigem Getreide auf die von Raupach (2012, S. 116) errechneten $189 \mu \mathrm{g} / \mathrm{kg}$ in Betracht. Auf diese Weise ließe sich die Gefahr einer TDIÜberschreitung von Kleinkindern deutlich reduzieren. Von den durch Renn (2008) vorgeschlagenen allgemeinen Managementstrategien für Risiken des Typs „Phythia“ erscheint außerdem die Entwicklung von Substituten geeignet, um den Gesundheitsschutz von Kindern vor DON zu verbessern. Vorstellbar wäre die Einführung spezieller Getreideprodukte für die Kinderernährung in Anlehnung an die von Raupach (2012, S. 131; S. 229f) vorgeschlagene Schaffung von Qualitätskategorien. Diese speziellen Kindergetreideprodukte dürften nur so viele Mykotoxine (DON) enthalten, dass ein Gesundheitsrisiko für Kinder sicher ausgeschlossen werden kann. In der Praxis könnten diese speziellen Kindergetreideprodukte vollständig gemäß der Diätverordnung für Baby- und Kleinkindernahrung (DiätV, 2010, §14) produziert werden. Dies hätte zum einen den Vorteil, dass bestehende Konzepte und Produktionsstrukturen für Vorzugsgetreide, welches auch für

\footnotetext{
${ }^{8}$ Siehe hierzu ausführlich: Raupach, 2012, S. $76 \mathrm{ff}$.

${ }^{9}$ Beispiele hierfür sind das Lebensmittel- und Warenkorbmonitoring, die Besondere Ernte- und Qualitätsermittlung sowie der Bundesweite Überwachungsplan.
} 
die Herstellung von Babynahrung verwendet wird, genutzt werden könnten. Zum anderen erhöht eine Vermarktung der speziellen Kindergetreideprodukte unter der allgemeinen Kennzeichnung für Baby- und Kleinkindernahrung vermutlich die Akzeptanz der Produkte unter Eltern.

Die erstgenannte Verbesserungsoption, die generelle Senkung der DON-Grenzwerte, stellt eine staatliche Maßnahme dar, auf deren Umsetzung die Eltern keinen unmittelbaren Einfluss haben. Der zweitgenannte Ansatz, die Einführung besonders gering mit Mykotoxinen belasteter Kindergetreideprodukte, kann als eine privat ausgerichtete Verbesserungsstrategie des DON-Risikomanagements aufgefasst werden, da Eltern selbst darüber entscheiden können, ob sie ihre Kinder mit besonders sicheren Getreideprodukten ernähren. Gemein ist beiden Verbesserungsansätzen, dass sie zu Preiserhöhungen bei Getreideprodukte führen, da die Vermeidung von Mykotoxinen mit Mehrkosten für die Lebensmittelunternehmer verbunden ist (Niens und Hasselmann, 2011). Während bei der ersten Option, der generellen Grenzwertsenkung, jedoch die Preise für alle Getreideprodukte in Deutschland steigen würden, erhöhten sich bei der Einführung besonders gering mit Mykotoxinen belasteter Kindergetreideprodukte nur die Preise für die besonders sicheren Lebensmittel. Dabei bietet die staatliche Verbesserungsmaßnahme jedoch den Vorteil, dass alle Kinder sicher vor einer Gesundheitsgefährdung durch DON geschützt wären. Nachteilig an der generellen Senkung der Mykotoxin-Grenzwerte ist jedoch, dass weniger anfällige Personen nicht mehr auf unter herkömmlichen Bedingungen produzierte und dadurch preisgünstigere Produkte zurückgreifen könnten. Hingegen hinge die Verbesserung des Gesundheitsschutzes von Kindern durch die Einführung besonders sicherer Kindergetreideprodukte von der Kaufentscheidung der Eltern ab. Somit kann ein vollständiger Gesundheitsschutz aller Kinder vor DON mithilfe der privat ausgerichteten Verbesserungsstrategie nicht garantiert werden.

\section{Grundlegende Fragestellungen und Zielsetzung der Arbeit}

Aus den Ausführungen der Kapitel 3 und 4 ergeben sich verschiedene Fragestellungen, die im Rahmen der vorliegenden Dissertation bearbeitet wurden. Diese werden nachstehend zusammengefasst: 
a) Ist eine Verbesserung des gesundheitlichen Verbraucherschutzes vor DON vor dem Hintergrund, dass die Vermeidung von Mykotoxinen mit einem Mehraufwand für die Lebensmittelunternehmer verbunden ist, aus volkswirtschaftlicher Sicht grundsätzlich sinnvoll? Dies wäre der Fall, wenn die volkswirtschaftlichen Kosten für einen umfassenden Verbraucherschutz vor DON durch seinen volkswirtschaftlichen Nutzen mindestens gedeckt würden.

b) Wie kann die Gesundheit von Kindern, die durch DON-Kontaminationen besonders gefährdet ist, ökonomisch bewertet werden und welche Probleme treten dabei auf?

c) Wünschen Eltern eine Verbesserung des gesundheitlichen Verbraucherschutzes vor Mykotoxinen (DON) und wenn ja, welche Regulierungsoption wird präferiert? Entweder eine generelle Senkung der Mykotoxin (DON)-Grenzwerte für alle Getreideprodukte in Deutschland oder die Einführung spezieller, kindergerechter Getreideprodukte, welche nur so viele Mykotoxine (DON) enthalten, dass ein Gesundheitsrisiko für Kinder sicher ausgeschlossen werden kann?

d) Wie wird das Gesundheitsrisiko für Kinder durch Mykotoxine (DON) von Eltern wahrgenommen?

e) Besteht eine Zahlungsbereitschaft für eine Verbesserung des Gesundheitsschutzes von Kindern vor Mykotoxinen (DON) auf Seiten der Eltern?

Zur Beantwortung der ersten Fragestellung a) wurden auf Basis von Feldversuchsdaten und von Richtwertdeckungsbeiträgen die variablen Kosten der Landwirtschaft, die eine Senkung des DON-Grenzwerts auf die von Raupach (2012) empfohlenen 189 Mg pro Kilo verzehrfertigem Getreide verursachen würde, berechnet. Diese wurden der von Freese und Enneking (2009) ermittelten Zahlungsbereitschaft niedersächsischer Konsumenten für ein Kilo besonders gering mit Mykotoxinen belastetes Weizenmehl gegenübergestellt, um Aussagen über die ökonomische Vorteilhaftigkeit einer Verbesserung des Verbraucherschutzes vor DON treffen zu können.

Die Bearbeitung der Fragestellung b) erfolgte auf Grundlage einer umfassenden Literaturanalyse. Hierzu wurden unter anderem das "Children's Health Valuation Handbook" der United States Environmental Protection Agency und ein Beitrag mit dem Titel „Review of methods and development of guidance for the economic valuation of transport-related 
health effects, with a particular focus on children", der im Auftrag der World Health Oraganization (WHO Europe) von Boesch et al. (2008) verfasst wurde, herangezogen.

Für die Fragestellungen c) bis e) wurden Eltern aus Niedersachsen mit Kindern zwischen drei und elf Jahren schriftlich zum Thema „Kinderernährung und Lebensmittelsicherheit in Deutschland“ befragt $^{10}$. Im Rahmen dieser Studie wurden die folgenden Konstrukte erhoben:

- Elterliche Regulierungspräferenzen des Mykotoxinrisikos: Zur Auswahl standen a) die generelle Senkung der Mykotoxin (DON)-Grenzwerte und b) die Einführung besonders gering mit Mykotoxinen belasteter Kindergetreideprodukte

- Elterliche Mykotoxin-Risikowahrnehmung: Einstufung des Mykotoxinrisikos hinsichtlich seiner Schwere und Charakterisierung des Risikos mithilfe des PFRIs

- Elterliche Zahlungsbereitschaft für spezielle Kindergetreideprodukte, die sicher nur so viele Mykotoxine enthalten, dass ein Gesundheitsrisiko für Kinder ausgeschlossen werden kann. Im Einzelnen: ein Kinder-Weizenbrötchen, ein Kinder-Mehrkornbrötchen, ein Kinder-Toastbrot und ein Kinder-Vollkornbrot

- Soziodemographische Merkmale von Eltern und Kindern: Alter und Geschlecht des Befragten sowie des Kindes, Bildungsstand des Befragten, Anzahl der Haushaltsmitglieder, Anzahl der Kinder unter 14 Jahren im Haushalt sowie das monatliche Haushaltsnettoeinkommen der Familie

\footnotetext{
${ }^{10}$ Der vollständige Fragebogen ist im Anhang dieser Arbeit einsehbar.
} 


\section{$6 \quad$ Literatur I}

Banasiak, U., Heseker, H, Sieke, C., Sommerfeld C. und Vohmann, C. (2005): Abschätzung der Aufnahme von Pflanzenschutzmittel-Rückständen in der Nahrung mit neuen Verzehrmengen für Kinder. Bundesgesundheitsblatt - Bundesgesundheitsforschung Gesundheitschutz 48 (1): 84-93.

Bartels, G. und Rodemann, B. (2003): Strategien zur Vermeidung von Mykotoxinen in Getreide. Gesunde Pflanzen 55 (5): 125-135.

Bennett, J.W. und Klich, M. (2003): Mycotoxins. Clinical Microbiology Reviews 16 (3): 497516.

BfR (Bundesinstitut für Risikobewertung) (2005): BfR entwickelt neues Verzehrsmodell für Kinder. Information Nr. 016/2005 des BfR vom 2. Mai 2005. URL: http://www.bfr.bund.de/cm/218/bfr_entwickelt_neues_verzehrsmodell_fuer_kinder.pdf (27.03.2008).

BgVV (Bundesinstitut für gesundheitlichen Verbraucherschutz und Veterinärmedizin) (2001): Die bovine spongiforme Enzephalopathie (BSE) des Rindes und deren Übertragbarkeit auf den Menschen. URL: http://www.bfr.bund.de/cm/208/ bse_des_rindes_und_deren_uebertragbarkeit_auf_den_menschen.pdf (09.04.2013).

BMELV (Bundesministerium für Ernährung, Landwirtschaft und Verbraucherschutz) (2012): Besondere Erne-und Qualitätsermittlung 2012. Reihe: Daten-Analysen. URL: www.bmelv-statistik.de (20.05.2013).

BMELV (Bundesministerium für Ernährung, Landwirtschaft und Verbraucherschutz) (2011): Statistisches Jahrbuch über Ernährung, Landwirtschaft und Forsten 2011. Landwirtschaftsverlag $\mathrm{GmbH}$, Münster.

Boesch, H.-J., Kahlmeier, S., Sommer, H., von Kempen, E., Staatsen, B. und Racioppi, F. (2008): Economic valuation of transport related health effects. Review methods and development of guidance for the economic valuation of transport-related health effects, with a particular focus on children. WHO Europe (World Health Organization, Regional Office for Europe), Denmark. URL: http://www.euro.who.int/pubrequest (28.09.2011). 
Büttner, P. (2006): Das Artenspektrum der Gattung Fusarium an Weizen und Roggen in Bayern in den Jahren 2003 und 2004. Gesunde Pflanzen 58: 28-33.

Curtui, V., Brockmeyer, A., Dietrich, R., Kappenstein, O., Klaffke, H., Lepschy, J., Märtlbauer, E., Schneider, E., Seidler, C., Thielert, G., Usleber, E., Weber, R. und Wolff, J. (2006): Analytik und Vorkommen wichtiger Fusarientoxine (Deoxynivalenol, Zearalenon) sowie Aufnahme dieser Toxine durch den deutschen Verbraucher. Abschlussbericht. Angewandte Wissenschaft 511.

Dehne, H.-W., Oerke, E.-C. und Steiner, U. (2002): Mykotoxine in Getreide und Getreideprodukten - Fusarium-Befall an Weizen. In: Beiträge zum ersten Workshop der Arbeitsgruppe Lebensmittelqualität und -sicherheit QUASI der Agrar- und Ernährungswissenschaftlichen Fakultät der Christian-Albrechts-Universität zu Kiel. Schriftenreihe der Agrar- und Ernährungswissenschaftlichen Fakultät der Universität Kiel, Heft 94. Selbstverlag der Agrar- und Ernährungswissenschaftlichen Fakultät der ChristianAlbrechts-Universität zu Kiel.

DiätV (Verordnung über Diätische Lebensmittel, Diätverordnung) (2010). URL: http://www.gesetze-im-internet.de/bundesrecht/di_tv/gesamt.pdf (29. 08. 2012).

FAO (Food and Agriculture Organization of the United Nations) (2004): Worldwide regulations for mycotoxins in food and feed in 2003. Food and Agriculture Organization of the United Nations, Rome 2004. URL: http://www.fao.org/docrep/ 007/y5499e/y5499e00.htm (10.04.2013).

Fife-Schaw, C. und Rowe, G. (2000): Extending the application of the psychometric approach for assessing public perceptions of food risk: some methodological considerations. Journal of Risk Research, 3 (2): 167-179.

Fife-Schaw, C. und Rowe, G. (1996): Public perceptions of everyday food hazards: a psychometric study. Risk Analysis, 16 (4): 487-500.

Fischhoff, B., Slovic, P., Lichtenstein, S., Read, S. und Combs, B. (2000): How safe is safe enough? A psychometric study of attitudes toward technological risks and benefits. In: Slovic, P., Löfstedt, R.E. (Editors). The perception of risk. Earthscan Publications, London. 
Freese, C. und Enneking, U. (2009): Erhebung zur Beurteilung und Verbesserung von Produktkennzeichnungsstrategien aus Sicht des Konsumentenverhaltens. Persönliche Mitteilung vom 14. April 2010.

Gareis, M. (1999): Mykotoxine und Schimmelpilze. ForschungsReport Ernährung Landwirtschaft Forsten 2/1999: 4-5.

Hansen, J., Holm, L., Frewer, L., Robinson, P. und Sandøe, P. (2003): Beyond the knowledge deficit: recent research into lay and expert attitudes to food risks. Appetite, 41: 111-121.

IARC (International Agency for Research on Cancer) (1993): IARC Monographs on the Evaluation of Carcinogenic Risks to Humans. Volume 56. Some Naturally Occurring Substances: Food Items and Constituents, Heterocyclic Aromatic Amines and Mycotoxins. Summary of Data Reported and Evaluation. URL: http://monographs.iarc.fr/ ENG/Monographs/vol56/volume56.pdf (17.05.07).

Kuiper-Goodman, T. (2004): Risk assessment and risk management of mycotoxins in food. In: Magan, N. und M. Olsen (Editors) (2004). Mycotoxins in food: detection and control. Woodhead Publishing Ltd., Cambridge, UK, 367-405.

Lebensmittel-Markt-Ernährung (2011): Nicht verzehrfähig: Zwei Bio-Nudelmarken mit Schimmelpilzgift. URL: http://www.animal-health-online.de/Ime/2011/03/24/nichtverzehrsfahig-zwei-bio-nudelmarken-mit-schimmelpilzgift/5888/ (04.04.2013).

Niens, C. und Hasselmann, H. (2011): Ist eine Verbesserung des Verbraucherschutzes vor Mykotoxinen ökonomisch rational?. Jahrbuch der Österreichischen Gesellschaft für Agrarökonomie - Band 20 (1): 181-190. Facultas Verlag, Wien.

Pieters, M. N., Freijer, J., Baars, B. J., Fiolet, D. C. M., Klaveren, J. Van und Slob, W. (2002): Risk assessment of Deoxynivalenol in food: concentration limits, exposure and effects. In: DeVries, J. W., Trucksess, M. W. und L. S. Jackson (Editors). Mycotoxins and food safety: Proceedings of an American Chemical Society symposium held in Washington, DC, USA, on 21-23 August 2000, 2002. Serie: Advances in Experimental Medicine and Biology. Kluwer Academic Publishers, 504: 235-248. 
Raupach, K. (2012): Risiko und Risikomanagement ausgewählter Fusarium-Mykotoxine. Eine Analyse mit dem Fokus auf der Verbesserung des gesundheitlichen Verbraucherschutzes. Ibidem-Verlag, Stuttgart.

Raupach, K. und Marggraf, R. (2011): Mykotoxine als Gesundheitsrisiko: Laien- und Expertensicht. Jahrbuch der Österreichischen Gesellschaft für Agrarökonomie - Band 20 (1): 191-200. Facultas Verlag, Wien.

Raupach, K. und Marggraf, R. (2012): Unzureichender Verbraucherschutz vor dem Mykotoxin Deoxynivalenol - Aktuelle Situation und Verbesserungsmöglichkeiten. URL: https://gewisola2012.unihohenheim.de/fileadmin/einrichtungen/gewisola2012/Beitraege /Raupach_Marggraf_GEWISOLA_2012.pdf (09.04.2013).

Reiß, J. (1976): Gefährdung der menschlichen Gesundheit durch Mykotoxine. Zeitschrift für Ernährungswissenschaft 15: 168-176.

Renn, O. (2008): Die Akzeptabilität von Risiken. Gefahrstoffe - Reinhaltung der Luft 68 (7/8): 289-293.

Renn, O. (2005): Risikokommunikation - Der Verbraucher zwischen Information und Irritation. In: TAB-Diskussionspapier Nr. 11 (2005),,Risikoregulierung bei unsicherem Wissen: Diskurse und Lösungsansätze“. Dokumentation zum TAB-Workshop „Die Weiterentwicklung des gesundheitlichen Verbraucherschutzes als ressortübergreifende Aufgabe“.

SCF (Scientific Committee on Food) (1999): Opinion of the Scientific Committee on Food on Fusarium toxins. Part 1: Deoxynivalenol (DON) (2. December 1999).

SCF (Scientific Committee on Food) (2002): Opinion of the Scientific Committee on Food on Fusarium toxins. Part 6: Group evaluation of T-2 toxin, HT-2 toxin, Nivalenol and Deoxynivalenol (adopted on 26 February 2002).

Sinn, H-W. (2003): Verbraucherschutz als Staatsaufgabe. Perspektiven der Wirtschaftspolitik 4 (2): 281-294.

Slovic, P. (1987): Perception of risk. Science, 236 (4799): 280-285. 
Slovic, P., Fischhoff, B. und Lichtenstein, S. (2000): Fact and Fears: Understanding perceived risk. In: Slovic, P. Löfstedt, R.E. (Editor) (2000): The perception of risk. Earthscan Publications Ltd, London.

Slovic, P., Fischhoff, B. und Lichtenstein, S. (2000a): Rating the risk. In: Slovic, P., Löfstedt, R.E. (Editor) (2000). The perception of risk. Earthscan Publications Ltd, London.

Starr, C. (1969): Social benefit versus technological risk. Science 165 (3899): 1232-1238.

U.S. Environmental Protection Agency (U.S. EPA), Office of Children's Health Protection; Office of Policy Economics and Innovation; National Center for Environmental Economics (2003): Children's Health Valuation Handbook. EPA 100-R-03-003. Washington, D.C.

Verordnung (EG) Nr. 1126/2007 der Kommission vom 28. September 2007 zur Änderung der Verordnung (EG) Nr. 1881/2006 zur Festsetzung der Höchstgehalte für bestimmte Kontaminanten in Lebensmitteln hinsichtlich Fusarientoxinen in Mais und Maiserzeugnissen.

Verordnung (EG) Nr. 178/2002 des europäischen Parlaments und Rates vom 28. Januar 2002 zur Festlegung der allgemeinen Grundsätze und Anforderungen des Lebensmittelrechts, zur Errichtung der Europäischen Behörde für Lebensmittelsicherheit und zur Festlegung von Verfahren zur Lebensmittelsicherheit.

Verordnung (EG) Nr. 1881/2006 der Kommission vom 19. Dezember 2006 zur Festsetzung der Höchstgehalte für bestimmte Kontaminanten in Lebensmitteln.

Verordnung (EWG) Nr. 315/93 des Rates vom 8. Februar 1993 zur Festlegung von gemeinschaftlichen Verfahren zur Kontrolle von Kontaminanten in Lebensmitteln.

Verreet, J-A. und J. Aumann (2002): Mykotoxinproblematik in der Pflanzenproduktion. In: Beiträge zum ersten Workshop der Arbeitsgruppe Lebensmittelqualität und -sicherheit QUASI der Agrar- und Ernährungswissenschaftlichen Fakultät der Christian-AlbrechtsUniversität zu Kiel. Schriftenreihe der Agrar- und Ernährungswissenschaftlichen Fakultät der Universität Kiel, Heft 94. Selbstverlag der Agrar- und Ernährungswissenschaftlichen Fakultät der Christian-Albrechts-Universität zu Kiel. 
WBGU (Wissenschaftlicher Beirat der Bundesregierung Globale Umweltveränderungen) (1999): Welt im Wandel: Der gesellschaftliche Umgang mit globalen Umweltrisiken. Jahresgutachten 1998. Springer, Berlin.

Weidenbörner, M. (1999): Lebensmittelmykologie. B. Behr's Verlag, Hamburg.

Working Party on National Environmental Policy (2005): The valuation of environmental health risks to children: Methodological and Policy Issues. ENV/EPOC/WPNEP (2004) 15/FINAL, OECD. URL: http://www.oecd.org/fr/environnement/outilsetevaluationdes politiquesdelenvironnement/35381312.pdf (30.01.2013).

Wu, F. (2006): Economic impact of Fumonisin regulations on global corn and peanut markets. In: Barug, D., Bhatnagar, D. Van Egmond, H.P., Van Der Kamp, J.W., Van Osenbruggen, W.A.und Visconti, A. (Editors) (2006): The Mycotoxin Factbook: Food and Feed Topics. Wageningen Academic Publishers, S. 83-93. 


\section{Gliederung und Inhalt der wissenschaftlichen Veröffentlichungen zum}

\section{Thema „Mykotoxine und Kindergesundheit"}

Zunächst wird allgemein der Frage nachgegangen, ob eine Verbesserung des gesundheitlichen Verbraucherschutzes vor Mykotoxinen (DON) aus ökonomischer Sicht sinnvoll ist. Danach erfolgt eine Fokussierung auf die Risikogruppe „Kind“. Dabei werden zunächst Probleme, welche in Zusammenhang mit der ökonomischen Bewertung von Kindergesundheit stehen, diskutiert. Danach werden die Ergebnisse einer Elternbefragung, die im Rahmen der vorliegenden Dissertation durchgeführt wurde, dargestellt. In dieser Studie wurden Eltern aus Niedersachen zu Ihrer Meinung über „Kinderernährung und Lebensmittelsicherheit in Deutschland" befragt, wobei der Schwerpunkt auf dem Gesundheitsrisiko durch Mykotoxine ${ }^{11}$ (DON) in Getreideprodukten lag. Die Darstellung der Befragungsergebnisse beginnt mit einem Beitrag zu den elterlichen Regulierungspräferenzen wobei zwei verschiedene Maßnahmen zur Verbesserung des Gesundheitsschutzes von Kindern vor Mykotoxinen bewertet wurden. Die angebotenen Verbesserungsoptionen waren a) die generelle Senkung der Mykotoxin-Grenzwerte für alle Getreideprodukte in Deutschland und b) die Bereitstellung spezieller, besonders gering mit Mykotoxinen belasteter Kindergetreideprodukte. Es schließen sich zwei Beiträge an, in deren Mittelpunkt die elterliche Risikowahrnehmung von Mykotoxinen und die elterliche Zahlungsbereitschaft für die Reduzierung des Gesundheitsrisikos von Kindern durch Mykotoxine stehen.

\footnotetext{
${ }^{11}$ Im Rahmen der Elternbefragung wurde allgemein von Mykotoxinen (Schimmelpilzgiften) gesprochen, um eine Überforderung der Befragten durch weitere Konkretisierung und neue Begrifflichkeiten zu vermeiden. Konkret beziehen sich die Elternbefragung und alle gegebenen Informationen zu Schimmelpilzgiften während der Befragung aber ausschließlich auf das Risiko für Kinder durch das Mykotoxin „Deoxynivalenol“ (DON).
} 


\subsection{Ist eine Verbesserung des Verbraucherschutzes vor Mykotoxinen ökonomisch} rational?

Niens, C. und Hasselmann, H. (2011): Ist eine Verbesserung des Verbraucherschutzes vor Mykotoxinen ökonomisch rational? Jahrbuch der Österreichischen Gesellschaft für Agrarökonomie - Band 20 (1): 181-190. Facultas Verlag, Wien. Verfügbar unter: http://oega.boku.ac.at/fileadmin/user upload/Tagung/2010/Band 20 1/18 Niens Hasselm ann 2010.pdf 


\section{Ist eine Verbesserung des Verbraucherschutzes vor Mykotoxinen ökonomisch rational?}

Is a higher level of consumer protection against mycotoxins a rational economic choice?

Christine NIENS und Heinrich HASSELMANN

\section{Zusammenfassung}

Beim Anbau von Getreide stellt der Befall mit Ährenfusarien ein erhebliches Qualitätsproblem dar. Fusarien können Mykotoxine erzeugen, deren langfristige Aufnahme gesundheitsschädigend wirkt. Daher unterliegt Getreide gesetzlichen Regelungen hinsichtlich des erlaubten Mykotoxingehalts. Allerdings gibt es Hinweise darauf, dass die derzeitige Grenzwertsetzung keinen umfassenden Verbraucherschutz garantiert. Im Folgenden wird ausgeführt, wie hoch Getreide maximal mit Mykotoxinen belastet sein darf, um gesundheitlich unbedenkliche Lebensmittel produzieren zu können. Ausgehend davon wird mittels Kosten-Nutzen-Analyse gezeigt, dass eine Verbesserung des Verbraucherschutzes vor Mykotoxinen ökonomisch rational ist.

Schlagworte: Mykotoxine, Verbraucherschutz, Kosten-Nutzen-Analyse

\section{Summary}

Fusarium infections are one of the main problems in wheat cultivation. Fusarium produces mycotoxins, which can cause serious, chronic health effects. To reduce consumers' health risks, wheat and wheat products underlie legal regulation concerning the maximum level of mycotoxin concentration. But legislated maximum levels do not prevent consumers from over-stepping acceptable total daily intake, particularly children. Therefore a new threshold is presented which en-

Erschienen im Jahrbuch der ÖGA, Band xx, Jahr xxxx. Diese Fußnote bitte nicht löschen! Fußnotentext [AGRAR Fußnotentext]. 
sures an improved consumer protection. By using cost-benefit-analysis, we show that a higher level of consumer protection against mycotoxins is a rational economic choice.

Keywords: mycotoxins, consumer protection, cost-benefit analysis

\section{Einleitung}

In der Landwirtschaft spielt neben einem hohen Ertrag die Qualität des Ernteguts eine wichtige Rolle. Beim Anbau von Getreide stellt besonders der Befall mit Feldpilzen der Gattung „Fusarium“ ein erhebliches Problem dar. Fusarien produzieren bestimmte, gesundheitsschädliche Schimmelpilzgifte, die "Mykotoxine“. Um eine Gesundheitsgefährdung der Verbraucher zu vermeiden, wurden in der EU MykotoxinHöchstgehalte für Getreide und getreidehaltige Lebensmittel gesetzlich festgelegt. Jedoch wird diskutiert, ob die aktuellen Grenzwerte einen ausreichenden Gesundheitsschutz gewährleisten.

Vor diesem Hintergrund wird gezeigt, welche Mykotoxingehalte $\mathrm{zu}$ einer Reduzierung der Gesundheitsgefährdung, insbesondere von Risikogruppen führen. Da eine Unterschreitung der aktuellen Grenzwerte für den Landwirt mit Mehraufwand und dadurch mit zusätzlichen Kosten verbunden ist, wird mittels einer Kosten-Nutzen-Analyse geprüft, ob eine Verbesserung des gesundheitlichen Verbraucherschutzes vor Mykotoxinen volkswirtschaftlich sinnvoll ist. Dabei steht die Ermittlung der Mehrkosten der Landwirte im Vordergrund.

\section{Mykotoxine im Getreide}

Unter den Getreidearten gilt Weizen als besonders anfällig für eine Fusariuminfektion. Der Pilzbefall führt zum Schadbild der "partiellen Taubährigkeit" und kann im schlimmsten Fall Ertragseinbußen bis zu $45 \%$ verursachen (vgl. RODEMANN und BARTELS, 2004). Zudem erzeugen Fusarien gesundheitsgefährdende Mykotoxine, wobei „Deoxynivalenol“ (DON) das häufigste Fusarium-Mykotoxin darstellt (vgl. RODEMANN und BARTELS, 2004). Aufgrund der weiten Verbreitung von DON beziehen sich alle weiteren Ausführungen auf dieses Mykotoxin und auf Weizen, als bedeutendstes Getreide für die Nahrungsmittelproduktion (vgl. BMELV, 2010). 


\section{Einflussfaktoren auf die Fusarium-Toxinbildung bei Weizen}

Für einen Befall des Weizens mit Fusarien sowie die DON-Bildung sind zum einen die Anbaustrategie des Landwirts und zum anderen die Witterungsverhältnisse zum Zeitpunkt der Weizenblüte entscheidend. $\mathrm{Zu}$ den wichtigsten pflanzenbaulichen Einflussfaktoren gehören Fruchtfolge, Sortenwahl, Bodenbearbeitung und der Einsatz spezieller Fungizide (vgl. BRUNOTTE, 2007).

- Fruchtfolge: Im Vergleich zu den Vorfrüchten Getreide und Blattfrucht erhöht sich der DON-Gehalt des Weizens nach Silomais um ca. $70 \%$. Hingegen sind keine signifikanten Unterschiede der Vorfruchtwirkungen von Getreide und Blattfrüchten in Bezug auf den DON-Gehalt der nachfolgenden Frucht nachweisbar (vgl. GÖDECKE und V. TIEDEMANN, 2009; BECK und LEPSCHY, 2000).

- Sortenwahl: Der Anbau einer hoch anfälligen Weizensorte erhöht die DON-Belastung des Getreides gegenüber einer gering anfälligen Sorte unter sonst identischen Anbaubedingungen um bis $\mathrm{zu} \mathrm{400 \%} \mathrm{(vgl.}$ GÖDECKE und V. TIEDEMANN, 2009).

- Bodenbearbeitung: Gegenüber einer Direktsaat können die DONGehalte durch eine wendende Bodenbearbeitung um ca. $70 \%$ und durch eine Mulchsaat mit Lockerung um ca. 50\% reduziert werden (vgl. BRUNOTTE, 2007). Der hohe Wirkungsgrad der wendenden Bodenbearbeitung wird allerdings nur in Kombination mit der Vorfrucht Mais erreicht (vgl. BARTELS und RODEMANN, 2003; BECK und LEPSCHY, 2000).

- Pflanzenschutzmittel: In Jahren mit günstigen Klimabedingungen für das Fusariumwachstum kann auf eine Blütenspritzung mit Azolfungiziden zurückgegriffenen werden, um die DON-Belastung des Weizens $\mathrm{zu}$ regulieren. Unter Praxisbedingungen wurden dadurch im Idealfall Reduzierungen zwischen $40 \%$ und $50 \%$ erreicht (vgl. BARTELS und RODEMANN, 2003; PAUL et al., 2008)

\section{Wirkungen von DON auf die Gesundheit}

Die Aufnahme von Mykotoxinen wie DON stellt ein gesundheitliches Risiko dar. Dabei wird Mykotoxinen vor allem ein chronisches Gefährdungspotential zugeschrieben (vgl. KUIPER-GODEMANN, 2004). Zu den durch DON ausgelösten Erkrankungen gehört die Immunsuppression, 
in deren Folge sich die Anfälligkeit für Infektionserkrankungen erhöht (vgl. SCF, 1999). Weiterhin kann DON auch Erbrechen, Übelkeit und Darmbluten hervorrufen (vgl. BARTELS und RODEMANN, 2003).

\subsection{Grenzwertsetzung und Gesundheitsschutz}

Die maximal zulässige Belastung von Weizen mit Mykotoxinen wird auf europäischer Ebene durch die Verordnung (EG) Nr. 1881/2006 geregelt. Die dort angegebenen Höchstgehalte sollen dem Schutz der öffentlichen Gesundheit dienen und bei Einhaltung guter Landwirtschaftspraxis erreichbar sein. Laut der Verordnung (EG) Nr. 1881/2006 darf unverarbeiteter Weizen maximal $1.250 \mu \mathrm{g}$ DON $/ \mathrm{kg}$ und Weizenmehl sowie direkt zum menschlichen Verzehr vorgesehener Weizen höchstens $750 \mu \mathrm{g}$ DON $/ \mathrm{kg}$ enthalten.

Die Ermittlung der Mykotoxin-Höchstgehalte erfolgt unter Bezugnahme des TDI (tolerable daily intake) und der täglichen Aufnahmemenge bestimmter Nahrungsmittel. Der TDI-Wert stellt die Menge eines potentiell gesundheitsschädlichen Stoffes dar, die lebenslang täglich konsumiert werden kann, ohne dass merkliche Gesundheitseffekte auftreten (vgl. KUIPER-GOODMAN, 2004). Für DON gilt ein TDI von $1 \mu \mathrm{g} / \mathrm{kg}$ Körpergewicht pro Tag (vgl. SCF, 2002).

\subsection{Empfehlungen für einen umfassenden Verbraucherschutz}

Es gibt Hinweise darauf, dass die aktuellen Grenzwerte eine Überschreitung des TDI bei bestimmten Personengruppen zulassen (vgl. BFR, 2006; Empfehlung der Kommission 2006/583/EG). RAUPACH und MARGGRAF (2009) zeigen, dass zwei- bis vierjährige Kinder mit durchschnittlichem Getreideverzehr den TDI um fast das Vierfache überschreiten können. Um eine Gesundheitsgefährdung von Kleinkindern zu reduzieren wird für Deutschland ein Grenzwert von $193 \mu \mathrm{g} / \mathrm{kg}$ für empfohlen (vgl. RAUPACH und MARGGRAF, 2009). Um verzehrfertiges Getreide mit diesem Grenzwert produzieren zu können, muss auch die DON-Belastung des Rohgetreides verringert werden. Bei der derzeitigen Grenzwertsetzung wird davon ausgegangen, dass sich die DONBelastung des Rohgetreides durch weitere Verarbeitungsschritte um ca. $40 \%$ reduziert. Übertragen auf den ermittelten DON-Höchstgehalt für verzehrfertiges Getreide von $193 \mu \mathrm{g} / \mathrm{kg}$, müsste der Grenzwert für Weichweizen als Rohgetreide also $321 \mu \mathrm{gg} / \mathrm{kg}\left((192,6 / 60)^{*} 100\right)$ betra- 
gen, was in etwa einem Viertel des gegenwärtig zulässigen Höchstgehaltes entspräche.

\section{5. Ökonomische Bewertung}

Eine Herabsetzung der zulässigen DON-Höchstgehalte kann für den Landwirt durch zusätzliche Maßnahmen zur Vermeidung eines Fusarienbefalls mit Mehrkosten verbunden sein. Andererseits resultiert aus einer Verbesserung des gesundheitlichen Verbraucherschutzes auch ein Nutzen für die Konsumenten, da Erkrankungen vermieden werden. Vor diesem Hintergrund wird mittels einer Kosten-NutzenAnalyse geprüft, ob ein umfassender Verbraucherschutz vor DON ökonomisch rational ist. Dafür müsste der volkswirtschaftliche Nutzen die volkswirtschaftlichen Kosten übertreffen. Entscheidend für die Vorteilhaftigkeit der Maßnahme ist aber weniger die absolute Höhe der Werte, sondern vielmehr das Vorzeichen der Kosten-Nutzen-Analyse. Da in jedem Jahr mit einem ähnlichen Verhältnis von Kosten und Nutzen strengerer DON-Grenzwerte zu rechen ist, ist für die Prüfung des Vorzeichens eine einjährige Betrachtung ausreichend.

Die volkswirtschaftlichen Kosten eines umfassenden Verbraucherschutzes entsprechen hier den Einkommensrückgängen der Landwirte, die aus der Produktion von Weizen mit einer maximalen DONBelastung von $321 \mu \mathrm{g} / \mathrm{kg}$ resultieren. Der volkswirtschaftliche Nutzen strenger Grenzwerte ergibt sich aus der Vermeidung von Krankheitskosten und Produktionsverlusten. Zudem ist mit intangiblen Effekten, wie einem erhöhten Sicherheitsgefühl der Konsumenten zu rechnen. Da jedoch unklar ist, wie viele Krankheitsfälle durch DON verursacht werden, ist es unmöglich die Anzahl vermiedener Erkrankungen zu kalkulieren. Folglich ist auch die Höhe der eingesparten Krankheitskosten und Produktionsverluste nicht bestimmbar. Der volkswirtschaftliche Nutzen wird daher über die Mehrzahlungsbereitschaft der Verbraucher für „mykotoxinreduzierte“ Nahrungsmittel ermittelt.

\subsection{Methodik: Bestimmung des volkswirtschaftlichen Nutzens}

Im Rahmen einer Konsumentenbefragung wurde die Zahlungsbereitschaft für einen umfassenden Verbraucherschutz vor Mykotoxinen erhoben. Hierzu wurde ein Fragebogen eingesetzt, mit dem die Zahlungsbereitschaft für ein Kilo Weizenmehl 405 mit unbedenklichem 
Mykotoxingehalt über eine offene Frage, direkt erfasst wurde. Die Zahlungsbereitschaft wurde 2009 in persönlichen Interviews am Point of Sale, in der Osnabrücker Innenstadt und bei Haustürbefragungen erfasst. Die Befragten wurden zuvor informiert, dass der aktuelle Mehlpreis ca. 40 Cent beträgt. Insgesamt haben 696 Personen eine Zahlungsbereitschaft geäußert (vgl. FREESE und ENNEKING, 2009).

\subsection{Methodik: Berechnung der volkswirtschaftlichen Kosten}

Zur Bestimmung der volkswirtschaftlichen Kosten sind die Einkommensrückgänge der Landwirte infolge des neuen DON-Grenzwerts von $321 \mu \mathrm{g} / \mathrm{kg}$ zu berechnen. Dazu werden unter Bezugnahme von Monitoringdaten (vgl. BRUNOTTE, 2007) und Feldversuchsergebnissen aus Südniedersachsen (vgl. IFZ, 2010) drei hypothetische Anbauszenarien und ein Basisszenario entworfen. Das Basisszenario beschreibt, eine besonders effiziente Methode, um Winterweizen (WW) entsprechend des aktuellen DON-Grenzwerts von $1.250 \mu \mathrm{g} / \mathrm{kg} \mathrm{zu}$ produzieren. Die Szenarien 1-3 ermöglichen die Produktion von WW mit maximal $321 \mu \mathrm{g}$ DON/kg. In allen Szenarien wird Weizen der Sorte „Cubus“ (mittlere Fusarienanfälligkeit) angebaut. Bezüglich des Mykotoxinrisikos wird von günstigen Wetterbedingungen für das Fusariumwachstum ausgegangen. Die DON-Belastung des WW im Basisszenario wurde von BRUNOTTE (2007) übernommen. Ausgehend davon wurden für die Szenarien 1-3 theoretische DON-Gehalte aufgrund der Fruchtfolgewirkung bei Direktsaat und einem Verzicht auf Blütenspritzung berechnet. Zur Erreichung eines DON-Gehalts des WW in Rohform von $321 \mu \mathrm{g} / \mathrm{kg}$ werden in Szenario 1-3 jeweils Vorfrucht, Bodenbearbeitung und Fungizideinsatz variiert (vgl. Tabelle 1).

Tab. 1: Darstellung verschiedener Anbauszenarien zur Produktion von WW

\begin{tabular}{|l|l|r|l|l|r|}
\hline & Fruchtfolge & $\begin{array}{c}\text { theoretischer } \\
\text { DON-Gehalt }\end{array}$ & $\begin{array}{c}\text { Boden } \\
\text { bearbeitung }\end{array}$ & $\begin{array}{c}\text { Blüten } \\
\text { spritzung }\end{array}$ & $\begin{array}{c}\text { Ertrag } \\
\text { dt/ha }\end{array}$ \\
\hline Basis & Raps/WW & $1050 \mu \mathrm{g} / \mathrm{kg}$ & Direktsaat & & 110 \\
\hline Sz. 1 & Silomais/WW & $1785 \mu \mathrm{g} / \mathrm{kg}$ & Pflug & $\begin{array}{l}\text { Folicur, } \\
\text { Caramba }\end{array}$ & 100 \\
\hline Sz. 2 & Raps/WW & $1050 \mu \mathrm{g} / \mathrm{kg}$ & Mulchsaat & $\begin{array}{l}\text { Folicur, } \\
\text { Caramba }\end{array}$ & 110 \\
\hline Sz. 3 & WW/WW & $1050 \mu \mathrm{g} / \mathrm{kg}$ & Mulchsaat & $\begin{array}{l}\text { Folicur, } \\
\text { Caramba }\end{array}$ & 90 \\
\hline
\end{tabular}

Quelle: Eigene Darstellung 
Über eine Teilkostenrechung werden auf Basis von Richtwertdeckungsbeiträgen (LWK NDS., 2010) für jedes Szenario Erlöse (je 50\% AWeizen zu 14, 00 Euro/dt und 50\% B-Weizen zu 13, 34 Euro/dt) und variable Kosten der Weizenproduktion pro Hektar errechnet. Für die Kostenkalkulation wird von einer durchschnittlichen Nährstoffversorgung der Böden (Gehaltsklasse C, LWK NDS., 2010) ausgegangen. Die Düngung erfolgt jeweils ertragsabhängig unter Berücksichtigung der Nährstoffrücklieferung der Vorfrucht. Des Weiteren wird ein intensiver chemischer Pflanzenschutz, einschließlich der Ausbringung von Insektiziden und Wachstumsreglern unterstellt (vgl. Tabelle 2).

Tab. 2: Deckungsbeiträge [EUR/ha] für WW mit unterschiedlichem DON-Gehalt

\begin{tabular}{|l|r|r|r|r|}
\hline & \multicolumn{1}{|c|}{ Basis } & \multicolumn{1}{c|}{ Szenario 1 } & \multicolumn{1}{c|}{ Szenario 2 } & \multicolumn{1}{c|}{ Szenario 3 } \\
\hline Saatgut & 64,30 & 64,30 & 64,30 & 64,30 \\
\hline Düngemittel & 422,50 & 598,45 & 422,50 & 457,00 \\
\hline Pflanzenschutz & 248,36 & 317,67 & 317,67 & 317,67 \\
\hline Var. masch. Kosten & 169,67 & 220,41 & 207,41 & 199,69 \\
\hline Sonstiges & 30,87 & 32,21 & 29,52 & 28,44 \\
\hline E var. Kosten [ha] & 935,70 & $1.233,04$ & $1.041,40$ & $1.067,10$ \\
\hline Erlöse & $1.503,70$ & $1.367,00$ & $1.503,70$ & $1.230,30$ \\
\hline Deckungsbeitrag [ha] & $\mathbf{5 6 8 , 0 0}$ & $\mathbf{1 3 3 , 9 6}$ & $\mathbf{4 6 2 , 3 0}$ & $\mathbf{1 6 3 , 2 0}$ \\
\hline
\end{tabular}

Quelle: LWK NDS., 2010; Eigene Darstellung

Die Einkommensrückgänge der Landwirte je Hektar ergeben sich aus den Differenzen zwischen dem Deckungsbeitrag des Basisszenarios und den jeweiligen Deckungsbeiträgen der Szenarien 1-3. Da der Ausmahlgrad von Weizenmehl Typ 405 zwischen $40 \%$ und 55\% beträgt, werden für die Herstellung von einem Kilo Mehl bis zu zweieinhalb Kilo Rohgetreide benötigt. Entsprechend müssen für die KostenNutzen-Analyse die Einkommensrückgänge des Landwirts bezogen auf zweieinhalb Kilo Rohgetreide berechnet werden.

\section{Ergebnisse und Diskussion}

Es zeigt sich, dass toxikologisch unbedenkliche DON-Grenzwerte, die einen verbesserten Gesundheitsschutz von Risikogruppen wie Kindern ermöglichen, bei guter landwirtschaftlicher Praxis und erhöhtem Fusariumdruck erreichbar sind (vgl. Tabelle 3). 
Tab. 3: DON des Weizens nach Bodenbearbeitung und Blütenspritzung

\begin{tabular}{|l|r|r|r|r|}
\hline Fruchtfolge & \multicolumn{3}{|c|}{ DON-Gehalt des Weizens als Rohware in $\boldsymbol{\mu g} / \mathbf{k g}$ nach: } \\
\hline & $\begin{array}{r}\text { Theoretische } \\
\text { Belastung }\end{array}$ & $\begin{array}{r}\text { Pflug } \\
(-70 \%)\end{array}$ & $\begin{array}{r}\text { Mulchsaat } \\
(-50 \%)\end{array}$ & $\begin{array}{r}\text { Blütenspritzung } \\
(-40 \%)\end{array}$ \\
\hline Szenario 1 & 1.785 & 535 & & $\mathbf{3 2 1}$ \\
\hline Szenario 2 & 1.050 & & 525 & $\mathbf{3 1 5}$ \\
\hline Szenario 3 & 1.050 & & 525 & $\mathbf{3 1 5}$ \\
\hline
\end{tabular}

Quelle: Eigene Darstellung

Die Anforderungen der VO (EG) Nr. 1881/2006 werden damit auch bei einem umfassenden Verbraucherschutz erfüllt. Die Einkommensrückgänge der Landwirte bezogen auf 2,5 Kilo Weizen mit maximal $321 \mu \mathrm{g}$ $\mathrm{DON} / \mathrm{kg}$, welche für ein Kilo mykotoxinreduziertes Mehl Typ 405 benötigt werden, betragen zwischen zwei Cent für Szenario 2 und zehn Cent für Szenario 1. Die Unterschiede begründen sich mit Ertragsdifferenzen und den jeweils erforderlichen pflanzenbaulichen Maßnahmen zur Erreichung des neuen DON-Grenzwerts von $321 \mu \mathrm{g} / \mathrm{kg}$. Die Zahlungsbereitschaft für ein Kilo Weizenmehl mit unbedenklichem Mykotoxingehalt beträgt durchschnittlich 68 Cent (vgl. FREESE und ENNEKING, 2009). Ausgehend von einem Mehlpreis von 40 Cent, der den Befragten im Rahmen der Datenerhebung als Status Quo vorgegeben wurde (vgl. Kap. 5.1), liegt die Mehrzahlungsbereitschaft der Konsumenten bei 28 Cent (vgl. Abbildung 1).

Abb. 1: Kosten und Nutzen eines umfassenden Verbraucherschutzes vor DON bezogen auf ein Kilo Weizenmehl Typ 405

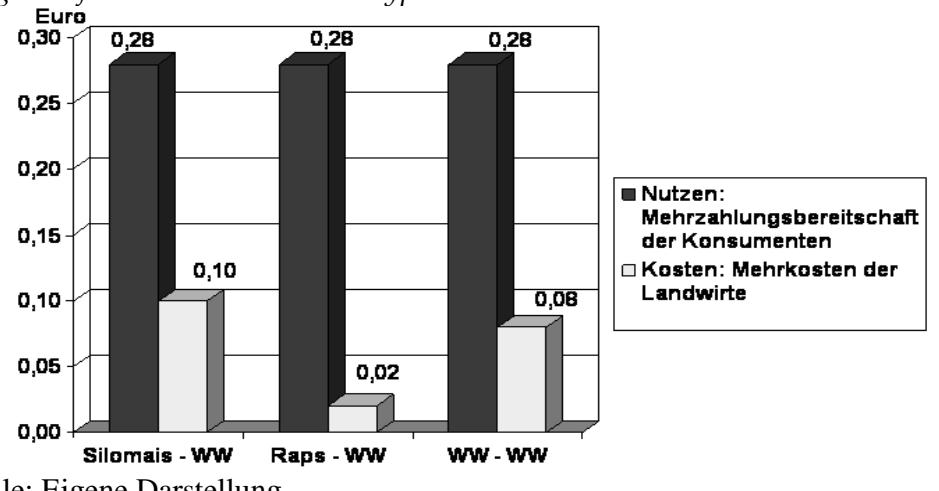

Quelle: Eigene Darstellung 
Der volkswirtschaftliche Nutzen (Mehrzahlungsbereitschaft der Konsumenten) übertrifft in allen Fällen die volkswirtschaftlichen Kosten (Mehrkosten der Landwirte) für strengere DON-Grenzwerte. Folglich stellt ein umfassender Verbraucherschutz vor dem Mykotoxin DON eine ökonomisch rationale Maßnahme dar.

$\mathrm{Zu}$ beachten ist allerdings, dass die ermittelten Kosten nur als Durchschnittwerte verstanden werden dürfen. Die einzelbetrieblichen Kosten können unter anderem durch standortbedingte Ertragsunterschiede abweichen. Des Weiteren können strengere DON-Grenzwerte auch zusätzliche Kosten für die weiterverarbeitende Industrie (Mühlen) verursachen. Hier könnte die alternative Verwendung von Winterweizen, welcher den Grenzwert überschreitet, kostenwirksam werden. Allerdings wurden auch auf der Nutzenseite nicht alle Nutzeneffekte berücksichtigt. So konnten die Einsparungen von Krankheitskosten sowie die Vermeidung von Produktionsverlusten nicht bestimmt werden. Daher ist insgesamt davon auszugehen, dass die volkswirtschaftlichen Kosten eines umfassenden Verbraucherschutzes vor DON durch den volkswirtschaftlichen Nutzen mindestens gedeckt werden.

\section{Danksagung}

Wir danken dem Niedersächsischen Ministerium für Wissenschaft und Kultur für die finanzielle Förderung des Forschungsvorhabens FAEN 3.

\section{Literatur}

BARTEls, G. und RoDEMANN, B. (2003). Strategien zur Vermeidung von Mykotoxinen in Getreide. Gesunde Pflanzen 55 (5), S. 125-135.

BECK, R. und LEPSCHY, J. (2000): Ergebnisse aus dem Fusarium-Monitoring 19891999 - Einfluß der produktionstechnischen Faktoren Fruchtfolge und Bodenbearbeitung. Schriftenreihe der Bayerischen Landesanstalt für Bodenkultur und Pflanzenbau, 3, S. 39-47.

BRUNOTTE J. (2007): Konservierende Bodenbearbeitung als Beitrag zur Minderung von Bodenschadverdichtungen, Bodenerosion, Run off und Mykotoxinbildung im Getreide. Landbauforschung Völkenrode. FAL Agricultural Research. Sonderheft 305. FAL Braunschweig.

BUNDESINSTITUT FÜR RISIKOBEWERTUNG (BFR) (2006): Erste Erfolge bei der Minimierung der Kontamination von Lebensmitteln mit dem Schimmelpilzgift Deoxynivalenol. Kurzprotokoll eines Expertengesprächs von 26. Januar 2006.

BUNDESMINISTERIUM FÜR ERNÄHRUNG, LANDWIRTSCHAFT UND VERBRAUCHERSCHUTZ (BMELV) (2010): Statistisches Jahrbuch über Ernährung, Landwirtschaft und Forsten 2009. 
EMPFEHLUNG DER KOMMISSION (2006/583/EG) vom 17.August 2006 zur Prävention und Reduzierung von Fusariumtoxinen in Getreide und Getreideprodukten.

FREESE, C. und ENNEKING, U. (2009): Erhebung zur Beurteilung und Verbesserung von Produktkennzeichnungsstrategien aus Sicht des Konsumentenverhaltens. Persönliche Mitteilung vom 14. April 2010.

GÖDECKE, R. und V. TIEDEMANN, A. (2009): Aktuelle Bewertung des Mykotoxinrisikos in Weizen. Getreidemagazin, 14, 2, S. 84-87.

INSTITUT FÜR ZUCKERRÜBENFORSCHUNG GÖTTINGEN (IFZ) (2010): Feldversuchsergebnisse 2009. Persönliche Mitteilung durch A. Muskolus.

KUIPER-GOODMANN, T. (2004): Risk assessment and risk management of mycotoxins in food. In: Magan, N. und M. Olsen (Eds): Mycotoxins in food: detection and control. Cambridge, UK, Woodhead Publishing Ltd., S. 367-405.

LANDWIRTSCHAFTSKAMMER NIEDERSACHSEN (LWK NDS.) (2010). RichtwertDeckungsbeiträge 2009. Oldenburg.

Paul, P. A., LipPs, P. E., Hershman, D. E., MCMullen, M. P., Draper, M. A. und MADDEN, L. V. (2008): Relative efficacy of triazole-based fungicides for Fusarium head blight and deoxynivalenol control in wheat. Phytopathology, 98, 9, S. 999-1011.

RAUPACH, K. und MARGGRAF, R. (2009): Verbraucherschutz vor dem Schimmelpilzgift Deoxynivalenol in Getreideprodukten. Diskussionsbeiträge des Departments für Agrarökonomie und Rurale Entwicklung der Georg-AugustUniversität Göttingen, Nr. 0904.

RODEMANN, B. und BARTELS, G. (2004): Fusariumbefall - Schadbild und Ausbreitung. Mais, 32, 1, S. 4-7.

SCIENTIFIC COMMITEE ON FOOD (SCF) (1999): Opinion on Fusarium Toxins Part 1: Deoxynivalenol (DON). Brüssel.

SCIENTIFIC COMMITEE ON FOOD (SCF) (2002). Opinion of the Scientific Committee on Food on Fusarium Toxins. Brüssel.

VERORDNUNG (EG) NR. 1881/2006 DER KOMMISSION vom 19. Dezember 2006 zu Festsetzung der Höchstgehalte für bestimmte Kontaminationen in Lebensmitteln.

\section{Anschrift der Verfasser}

Dipl. Sozw. Christine Niens und M. Sc. Heinrich Hasselmann Georg-August-Universität Göttingen Platz der Göttinger Sieben 5,37073 Göttingen, Deutschland Tel.: +49 (0)551 4853

eMail:cniens@uni-goettingen.de 


\section{2 Ökonomische Bewertung von Kindergesundheit in der Umweltpolitik - Aktuelle} Ansätze und ihre Grenzen

Niens, C. und Marggraf, R. (2010): Ökonomische Bewertung von Kindergesundheit in der Umweltpolitik - Aktuelle Ansätze und ihre Grenzen. Diskussionspapiere des DARE Nr. 1005. Verfügbar unter : http://www.uni-goettingen.de/de/72592.html 


\section{GEORG-AUGUST-UNIVERSITÄT}

GÖTTINGEN

August 2010

\section{Diskussionspapiere}

Discussion Papers

\section{Ökonomische Bewertung von Kindergesundheit in der Umweltpolitik}

Aktuelle Ansätze und ihre Grenzen

Christine Niens und Rainer Marggraf

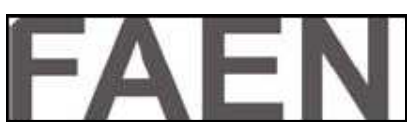

Forschungsverbund

Agrar- und Ernährungswissenschaften Niedersachsen

Gefördert durch Das Niedersächsische

Department für Agrarökonomie und Rurale Entwicklung

Universität Göttingen

D 37073 Göttingen

ISSN 1865-2697 


\section{Kontaktadressen:}

Dipl.-Sozw. Christine Niens

Abteilung Umwelt- und Ressourcenökonomik

Department für Agrarökonomie und Rurale Entwicklung

Georg-August-Universität Göttingen

Platz der Göttinger Sieben 5

D-37073 Göttingen

E-Mail: cniens@uni-goettingen.de

Tel.: +49 (0)551 - 394853

Fax: +49 (0)551 - 394812

Prof. Dr. Rainer Marggraf

Abteilung Umwelt- und Ressourcenökonomik

Department für Agrarökonomie und Rurale Entwicklung

Georg-August-Universität Göttingen

Platz der Göttinger Sieben 5

D-37073 Göttingen

E-Mail: rmarggr@uni-goettingen.de

Tel.: +49 (0)551 - 394829

Fax: +49(0)551 - 394812

\section{Danksagung}

Die Arbeit ist entstanden im Rahmen des dritten Forschungsverbundes Agrar- und Ernährungswissenschaften Niedersachsen (FAEN): „Qualitätsgerechte Pflanzenproduktion unter veränderten Rahmenbedingungen: Mykotoxine im Kontext von Produktion, Qualität und Verarbeitung“. Wir danken dem Niedersächsischen Ministerium für Wissenschaft und Kultur für die finanzielle Förderung des Forschungsvorhabens. 


\section{Inhalt}

$1 \quad$ Einleitung .............................................................................................................................. 1

2 Gründe für den besonderen Schutz von Kindergesundheit ........................................2

$2.1 \quad$ Rechtliche und politische Fundierung ........................................................... 2

2.2 Risikounterschiede zwischen Kindern und Erwachsenen ........................................ 3

2.2.1 Erhöhte Anfälligkeit von Kindern aufgrund körperlicher Voraussetzungen ..... 3

2.2.2 Erhöhte Anfälligkeit von Kindern aufgrund verstärkter Exposition .................. 3

2.2.3 Kumulative Wirkungszusammenhänge...................................................... 5

2.3 Zum volkswirtschaftlichen Nutzen des Schutzes von Kindergesundheit ..................5

3 Ökonomische Bewertungskriterien ..................................................................6 6

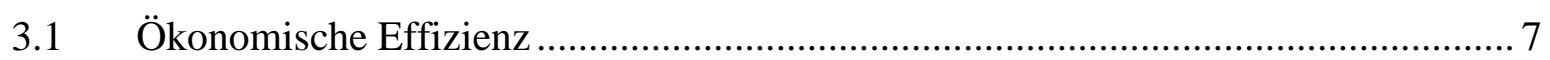

3.2 Zur Ermittlung von Kosten und Nutzen bei Kindergesundheitsprojekten ................ 9

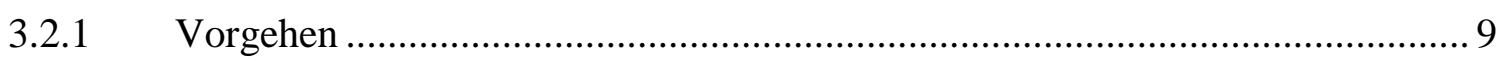

3.2.2 Schwierigkeiten bei der Erhebung von Zahlungsbereitschaften ..................... 11

4 Herausforderungen bei der ökonomischen Bewertung von Kindergesundheit ....... 12

4.1 Die Erhebung von Zahlungsbereitschaften über Stellvertreter ............................... 13

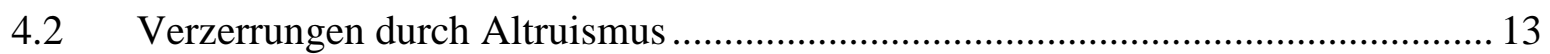

4.3 Verzerrungen infolge von Effekten sozialer Erwünschtheit ................................ 15

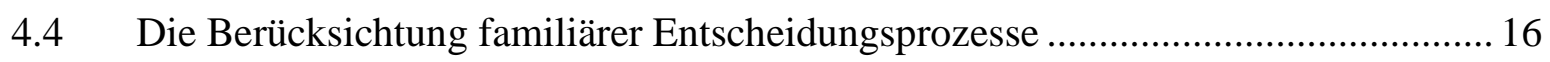

5 Aktuelle Entwicklung in der Umwelt- und Gesundheitspolitik ................................. 19

6 "Pan-European Programme on Transport, Health and Environment" ................... 20

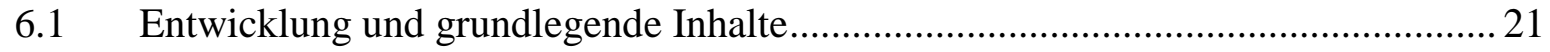

6.2 Zur Ermittlung verkehrsbedingter Krankheitskosten ........................................... 22

6.2.1 Die Kostenkalkulation entsprechend des PEPa.............................................. 22

6.3 Herausforderungen bei der Monetarisierung verkehrsbedingter Erkrankungen ...... 25

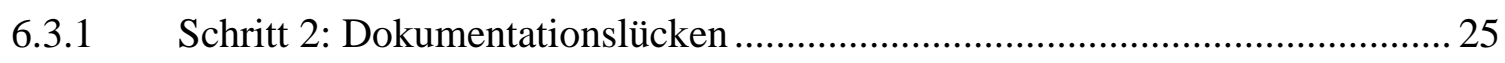

6.3.2 Schritt 3: Unsicherheiten aufgrund fehlender Daten...................................... 26

6.3.3 Schritt 3: Die Berücksichtigung von Kindern ............................................... 27

6.3.4 Schritt 4: Probleme bei der Bewertbarkeit bestimmter Gesundheitseffekte .... 28

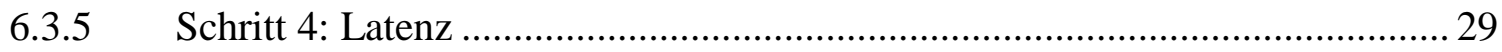

6.3.6 Schritt 4: Die Berücksichtigung von Kindern .............................................. 29

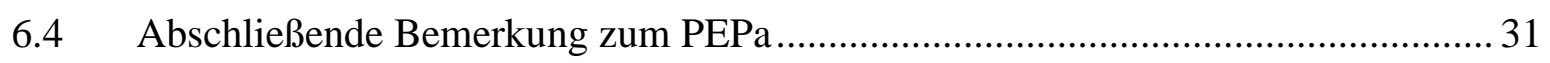

7 “Children's Health Valuation Handbook" ................................................................ 32

7.1 Entwicklung und grundlegende Inhalte.......................................................... 32 
7.2 Vorgehen bei der Nutzenerfassung von Kindergesundheitsprojekten 33

7.3 Risikounterschiede zwischen Kindern und Erwachsenen ...................................... 34

7.4 Ermittlung des Nutzens über die Zahlungsbereitschaft....................................... 35

7.4.1 Methoden zur indirekten Bestimmung einer WTP ....................................... 36

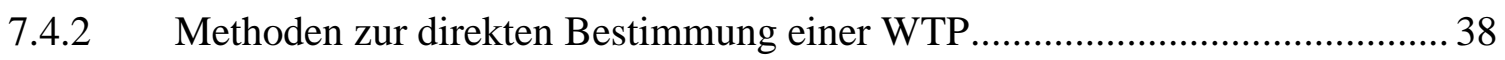

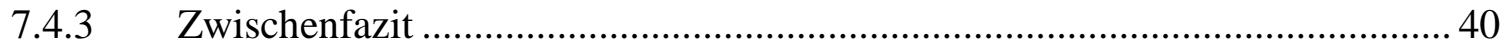

7.5 Ermittlung des Nutzens mittels des Krankheitskostenansatzes............................... 41

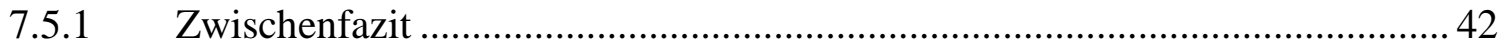

7.6 Bewertungsunterschiede zwischen Kindern und Erwachsenen ............................. 42

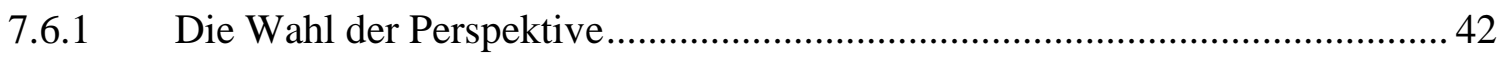

7.6.2 Unterschiede bei WTP und COI zwischen Kindern und Erwachsenen ............ 44

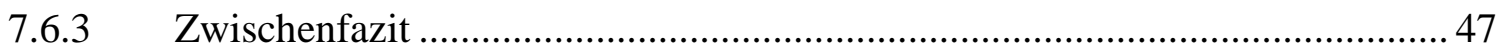

7.7 Alternative Ansätze zur Bewertung umweltpolitischer Kindergesundheitsprojekte 47

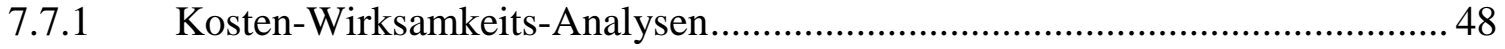

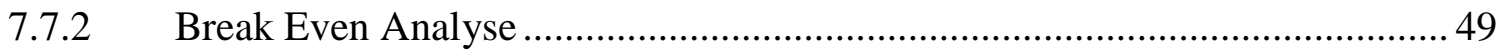

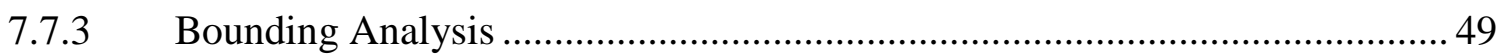

7.7.4 Risk-Risk- and Health-Health-Analysis...................................................... 50

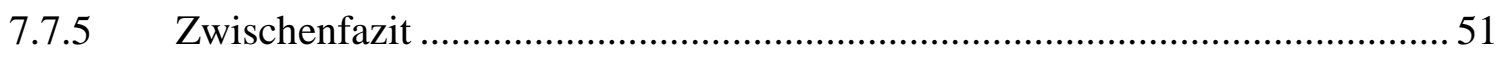

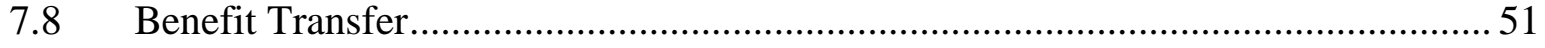

7.8.1 Phase 1: Beschreibung des umweltpolitischen Problems ................................ 52

7.8.2 Phase 2: Beurteilung der Brauchbarkeit vorhandener Untersuchungen............55

7.8.3 Phase 3: Übertragung der Daten................................................................ 57

7.8.4 Phase 4: Qualitative Beschreibung von Unsicherheiten.................................. 59

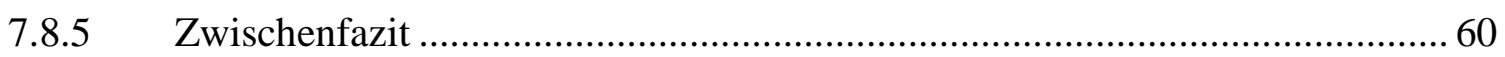

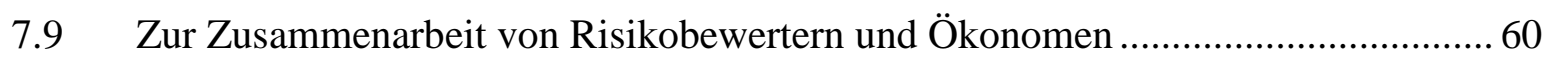

7.10 Abschließende Bemerkung zum „Children’s Health Valuation Handbook“........... 61

8 Vergleich von PEPa und ,Children’s Health Valuation Handbook ${ }^{66}$.......................... 63

9 Abschließende Bemerkung ............................................................................................................ 65

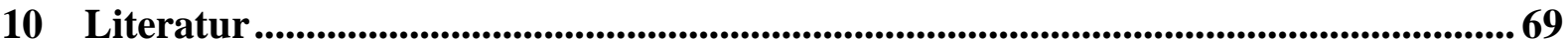


Abkürzungsverzeichnis

Abkürzung

COI

DALYs

OECD

PEP

PEPa

QALYs

U.S. EPA

VERHI

VLYL

VSL

WHO

WTA

WTP

\section{Bedeutung}

Costs of Illness

Disability-Adjusted Life Years

Organisation for Economic CO-Operation and Development

Transport, Health and Environment Pan-

European Programme

Review methods and development of

guidance for the economic valuation of

transport-related health effects, with a

particular focus on children

Quality-Adjusted Life Years

U.S. Environmental Protection Agency

Valuation of Environment-Related Health

Impacts with a Particular Focus on Children

Value of Life Years Lost

Value of Statistical Life

World Health Organization

Willingness to Accept

Willingness to Pay

\section{Abbildungsverzeichnis}

Abbildung 1: Komponenten des volkswirtschaftlichen Nutzens von

Kindergesundheitsprojekten.

Abbildung 2: Anpassung des Wohlstandsniveaus der aktuellen Erwachsenengeneration an zukünftige Erwachsenengenerationen

Abbildung 3: Methoden zur Bestimmung des volkswirtschaftlichen Nutzens umweltpolitischer Kinderschutzprojekte. 


\section{Tabellenverzeichnis}

Tabelle 1: Grundsätzliches Vorgehen zur Ermittlung umweltbedingter Krankheitskosten..... 22

Tabelle 2: Ermittlung des Nutzens von umweltpolitischen Gesundheitsprojekten................... 34

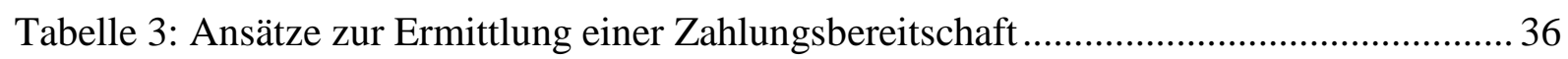

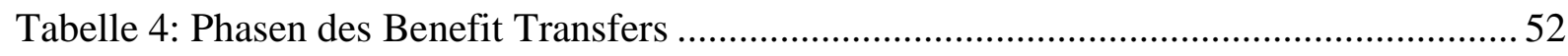




\section{$1 \quad$ Einleitung}

Seit dem Ende der 1980er Jahre wird dem Zusammenhang von Umwelt und menschlicher Gesundheit zunehmend Aufmerksamkeit geschenkt. Die Tatsache, dass Umweltbelastungen zu gesundheitlichen Problemen führen können, wurde erkannt und man begann auf politischer Ebene Maßnahmen zur Risikoreduktion zu entwickeln. Parallel dazu setzte sich auch die Wissenschaft vermehrt mit den Wechselwirkungen zwischen Umwelt und menschlicher Gesundheit auseinander. Hierbei zeigte sich, dass bestimmte Bevölkerungsgruppen besonders sensibel auf negative Umweltreize reagieren. Zu diesen Risikogruppen gehören in erster Linie Kinder, welche aufgrund ihrer Physiologie und ihrer spezifischen Verhaltsweisen oft gefährdeter sind als Erwachsene ${ }^{1}$.

Inzwischen existiert eine Vielzahl politischer Programme, die sich mit dem Thema „Umwelt und Gesundheit“" und insbesondere der Gefährdung von Kindern beschäftigen. Aktuell wird zunehmend eine stärkere Kooperation zwischen Politik und Wissenschaft angestrebt, unter anderem um die Wirksamkeit politischer Maßnahmen ökonomisch bewerten zu können.

Vor diesem Hintergrund wird im Folgenden auf die ökonomische Bewertung von Kindergesundheit eingegangen. Hierzu wird gezeigt, wie Kosten und Nutzen einer Verringerung umweltbedingter Gesundheitsrisiken für Kinder gemessen werden können. In diesem Zusammenhang wird dargelegt, welche Rolle ökonomische Bewertungsmodelle in der aktuellen Umwelt- und Gesundheitspolitik spielen und welche Probleme mit der Durchführung solcher Wirtschaftlichkeitsuntersuchungen verbunden sind. Schließlich werden Grenzen der zurzeit in der Politik angewandten, ökonomischen Bewertungsansätze offen gelegt.

Beginnen wird dieser Beitrag mit einer Darstellung der grundlegenden Aspekte, die den besonderen Schutz von Kindergesundheit dringend erforderlich erscheinen lassen. Danach wird auf die Notwendigkeit der Durchführung von Wirtschaftlichkeitsuntersuchungen in der Politik und auf die Kosten-Nutzen-Analyse als ein mögliches Verfahren zur ökonomischen Bewertung von Kindergesundheit eingegangen. Anschließend folgt ein Überblick über Empfehlungen zur praktischen Durchführung ökonomischer Bewertungen im umwelt- und gesundheitspolitischen Kontext mit einer besonderen Ausrichtung auf Kinder. Daran schließt sich eine vergleichende Bewertung dieser Ansätze an.

\footnotetext{
${ }^{1}$ Als Kinder werden in diesem Beitrags alle Personen bezeichnet, die das 18. Lebensjahr noch nicht vollendet haben.
} 


\section{Gründe für den besonderen Schutz von Kindergesundheit}

Es gibt vielfältige Gründe dafür, die Gesundheit von Kindern in besonderem Maße vor umweltbedingten Risikofaktoren zu schützen. Zum einen begründen sich die gesteigerten Bemühungen um den Schutz von Kindergesundheit mit rechtlichen Vereinbarungen. Zum anderen bewirkt eine erhöhte Anfälligkeit von Kindern gegenüber umweltbedingten Gesundheitsgefahren ein verstärktes Engagement entsprechende Risiken zu verringern. Schließlich ist die Sicherstellung eines guten gesundheitlichen Zustands der Kinder auch aus gesamtgesellschaftlicher Sicht vorteilhaft.

In diesem Abschnitt werden die Argumente für die besondere Schutzwürdigkeit von Kindergesundheit dargestellt

\subsection{Rechtliche und politische Fundierung}

Die UN Kinderrechtskonvention (Convention on the Rights of the Child) bildet eine internationale Rechtsgrundlage für den besonderen Schutz des Kindes. Sie wurde 1989 von der UN Generalversammlung angenommen und trat im Jahr 1990 in Kraft. Mit dieser Erklärung wurde zum ersten Mal in der Geschichte ein völkerrechtlich verpflichtendes Dokument für Kinder und Jugendliche auf internationaler Ebene vereinbart.

Insgesamt haben 193 Staaten, jedoch nicht die USA und Somalia, der Erklärung zugestimmt (United Nations Treaty Collection). Die Vertragsstaaten verpflichten sich damit, die in dem Dokument genannten Kinderrechte im Rahmen ihrer nationalen Gesetze zu verwirklichen.

Mit der Ratifizierung gehen die Vertragspartner auch verschiedene Pflichten hinsichtlich der Gesundheit von Kindern ein. So wird in Artikel 24 Abs.1 der Kinderrechtskonvention das Recht des Kindes auf ein „erreichbares Höchstmaß an Gesundheit“ anerkannt. Folglich muss dieses Recht des Kindes bei der Entwicklung umwelt- und gesundheitspolitischer Maßnahmen berücksichtigt werden.

Annährend zeitgleich wurde im Jahr 1989 die erste Europäische Ministerkonferenz „Umwelt und Gesundheit“ in Frankfurt durchgeführt, bei der die „Europäische Charta Umwelt und Gesundheit“" verabschiedet wurde. In diesem Abkommen heißt es: „Jeder Mensch hat Anspruch auf eine Umwelt, die ein höchstmögliches Maß an Gesundheit und Wohlbefinden ermöglicht" (Weltgesundheitsorganisation, Büro für Europa). Die Charta markiert den Beginn der vermehrten Auseinandersetzung mit dem Zusammenspiel von Umweltkontaminationen und daraus resultierenden Gesundheitseffekten. 
Die UN Kinderrechtskonvention und die „Europäische Charta Umwelt und Gesundheit“ bilden damit gemeinsam den Ausgangspunkt für die Auseinandersetzung mit den Thema „Umwelt und Kindergesundheit“ auf politischer Ebene in Europa.

\subsection{Risikounterschiede zwischen Kindern und Erwachsenen}

Die Gesundheit von Kindern ist im besonderen Maße von der Umweltsituation abhängig. In der Literatur gibt es zahlreiche Hinweise darauf, dass Kindergesundheit stärker als die von Erwachsenen durch Umweltgefahren bedroht ist (TAMBURLINI, 2006: 37ff; BOESCH et al., 2008: 12; Umweltbundesamt). Diese Risikounterschiede beziehen sich zum einen auf die Anfälligkeit und zum anderen auf die Exposition gegenüber bestimmten Umweltgefahren. Dies führt dazu, dass die Gesundheitseffekte von Kindern in quantitativer und qualitativer Hinsicht von denen eines Erwachsenen abweichen können.

\subsubsection{Erhöhte Anfälligkeit von Kindern aufgrund körperlicher Voraussetzungen}

Die erhöhte Anfälligkeit von Kindern gegenüber negativen Umwelteinflüssen resultiert vor allem aus abweichenden physischen Grundvorrausetzungen. Dieses kann sich sowohl auf die Schwere als auch auf die Häufigkeit gesundheitlicher Beeinträchtigungen auswirken. Die qualitativen Differenzen in Bezug auf bestimmte gesundheitliche Folgen beruhen auf der Tatsache, dass sich Kinder in einem dynamischen Wachstumsprozess befinden, in dem lebenswichtige Organe noch nicht vollständig entwickelt sind. In dieser Phase reagiert der Körper besonders sensibel auf bestimmte Umweltgifte. Dadurch kann es zu erheblichen Organ- und Entwicklungsschäden bei Kindern kommen, die bei Erwachsenen nicht zu erwarten sind (TAMBURLINI, 2006: 40).

Des Weiteren unterscheidet sich der Stoffwechsel eines Kindes von dem eines Erwachsenen. Daher können zum Beispiel bestimmte Pestizide von Kindern nicht ausreichend abgebaut werden (Chamley und PutZrath, 2001). Infolgedessen kommt es bei Kindern vergleichsweise häufiger zu Erkrankungen durch diese Umweltgifte (TAMBURLINI, 2006: 39f).

\subsubsection{Erhöhte Anfälligkeit von Kindern aufgrund verstärkter Exposition}

Die Expositionsunterschiede zwischen Kindern und Erwachsenen beziehen sich in erster Linie auf die gesteigerte Anzahl von Erkrankungen durch gesundheitsgefährdende Umwelteinflüsse (TAMBURLINI, 2006: 41). Diese Differenzen hinsichtlich der Belastungshäufigkeit werden einerseits durch biologische Faktoren hervorgerufen, welche charakteristisch für die menschliche Wachstumsphase sind. Anderseits führen bestimmte, für 
Kinder typische, Verhaltensweisen in der Phase der geistigen Entwicklung zu einer erhöhten Gefährdung durch die Umwelt.

Das besondere, biologische begründete Risiko von Kindern beruht auf einer im Vergleich zu Erwachsenen höhere Aufnahmemenge von Nahrung, Wasser und Luft pro Kilogramm Körpergewicht. Außerdem haben Kinder häufig spezifische Ernährungsmuster, bei denen bestimmte Lebensmittel überdurchschnittlich stark konsumiert werden (BANASIAK et al., 2005). Sind diese Lebensmittel mit schädlichen Stoffen belastet, erhöht dies die Wahrscheinlichkeit einer Gesundheitsschädigung (TAMBURLINI, 2006: 42).

Des Weiteren kann eine verstärkte Exposition auch auf einer gesteigerten Absorptionsfähigkeit von Kindern in der Phase der körperlichen Entwicklung beruhen. So sind Kinder beispielsweise in der Lage, mehr Blei über den Organismus aufzunehmen als Erwachsene (ROYCE, 1992). Dadurch ist bei Kindern mit einer vermehrten quantitativen Belastung durch Bleirückstände in der Umwelt zu rechnen.

Ein weiterer wichtiger Grund für eine erhöhte Gefährdung von Kindergesundheit durch Umweltrisiken ist die längere verbleibende Lebensspanne junger Menschen im Vergleich zu Älteren. Dadurch sind Kinder vergleichsweise länger einer Belastung mit gesundheitsschädlichen Stoffen ausgesetzt und es steht mehr Zeit zur Verfügung, um Krankheiten zu entwickeln (TAMBURLINI, 2006: 42). Dieser Aspekt ist besonders für Umweltrisiken relevant, die sich verzögert auf die Gesundheit auswirken.

Die auf spezifischen Verhaltensweisen beruhende erhöhte Gefährdung von Kindern entsteht häufig aufgrund abweichender Handlungsmuster in Risikosituationen. HARBAUGH et al. (2002) weisen experimentell nach, dass Kinder und Jugendliche dazu neigen, kleine Wahrscheinlichkeiten zu unter- und große Wahrscheinlichkeiten zu überschätzen. Das führt dazu, dass Kinder in Situationen, in denen mit geringen Wahrscheinlichkeiten große Verluste zu erwarten sind, risikobereiter handeln als Erwachsene (HARBAUGH et al., 2002: 73). Dieser Befund ist für den Bereich „Umwelt und Gesundheit“ besonders interessant, da man es hier häufig mit geringen Eintrittswahrscheinlichkeiten für schwerwiegende Erkrankungen zu tun hat. Demnach scheinen sich Kinder den umweltbedingten Gesundheitsgefahren oft nicht in dem Maße bewusst zu sein wie Erwachsene, was dazu führen kann, dass sie mit Risiken nicht in gleicher Weise umgehen.

Weiterhin unterscheiden sich besonders Kleinkinder von Erwachsenen erheblich hinsichtlich ihrer alltäglichen Verhaltensweisen. So nehmen Kleinkinder durch das Krabbeln am Boden oder während der oralen Phase bestimmte bodennahe Umweltgifte auf, denen Erwachsene in 
wesentlich geringerem Maße ausgesetzt sind (TAMBURLINI, 2006: 42). Zusätzlich halten sich Kinder aufgrund ihrer geringeren Körpergröße näher am Boden auf und atmen dadurch beispielsweise vermehrt Autoabgase ein (BOESCH et al., 2008: 12).

Aus der Gesamtheit dieser besonderen Verhaltensmuster ergibt sich ein spezifisches Expositionsprofil für Kinder. Dieses führt dazu, dass ihre Gesundheit häufiger durch schädliche Umwelteinflüsse bedroht ist als die von Erwachsenen.

\subsubsection{Kumulative Wirkungszusammenhänge}

Eine weitere Verschärfung der Risikounterschiede zwischen Kindern und Erwachsen ist zu erwarten, wenn erhöhte Anfälligkeit und vermehrte Exposition in Kombination auftreten. Dies ist zum Beispiel bei organophosphaten Pestiziden oder Blei der Fall (TAMBURLINI, 2006: 44). Zum einen reagieren Kinder besonders sensibel auf diese Umweltgifte. Zum anderen sind sie diesen Stoffen besonders stark ausgesetzt. Diese kumulative Wirkung bestimmter Umweltgefahren kann sich sowohl auf die Anzahl der betroffen Kinder als auch auf die Schwere der resultierenden Gesundheitseffekte auswirken.

\subsection{Zum volkswirtschaftlichen Nutzen des Schutzes von Kindergesundheit}

Die Vermeidung umweltbedingter Erkrankungen bei Kindern hat einen vielfältigeren Nutzen, als es auf den ersten Blick der Fall zu sein scheint. Zunächst tragen die Bemühungen, umweltbedingte Gesundheitsgefahren $\mathrm{zu}$ reduzieren dazu bei, gesundheitliche Beeinträchtigungen von Kindern in qualitativer und quantitativer Hinsicht zu verringern. An diesen Effekt sind aber noch eine Reihe weiterer Nutzenaspekte geknüpft. So ergibt sich der volkswirtschaftliche Gesamtnutzen des Schutzes von Kindergesundheit einerseits aus dem materiellen Nutzen, welcher sich weiter in direkte und indirekte materielle Größen aufgliedern lässt. Andererseits besitzen wirksame Regelungen zum Schutz von Kindergesundheit auch eine immaterielle Komponente, welche einen beachtlichen Anteil des volkswirtschaftlichen Gesamtnutzens ausmachen kann.

Direkter materieller Nutzen von Kindergesundheitsprojekten ergibt aus den Einsparungen durch eine Verminderung der Ausgaben für ambulante oder stationäre Behandlungen (GREINER, 2007: 53). Ein weiterer positiver Effekt besteht darin, dass berufstätige Eltern ihre Kinder seltener beaufsichtigen müssen, wenn diese seltener erkranken, wodurch sich die Anzahl der Fehltage am Arbeitsplatz verringert. Dies wiederum hat zur Folge, dass Produktionsverluste vermieden werden, was dem indirekten materiellen Nutzen gleichkommt. Andere indirekte materielle Nutzenaspekte bestehen darin, dass gesunde Kinder bessere 
Bildungschancen besitzen und dadurch verstärkt in der Lage sind, einen positiven Beitrag zum Humankapital zu leisten (Hoffmann, 2007: 218f). Somit ist ein guter gesundheitlicher Zustand der Kinder einer Gesellschaft auch aus wirtschaftlicher Sicht von Interesse.

Immaterieller Nutzen von Kindergesundheitsprojekten entsteht, weil Einschränkungen der Lebensqualität von Kindern und Eltern vermieden werden. Konkret ergeben sich positive immaterielle Effekte für Kinder, da Minderungen des persönlichen Wohlergehens durch Krankheit ausbleiben. Infolge dessen müssen sich Eltern weniger Sorgen um ihr erkranktes Kind machen. Fehlen hingegen besondere Maßnahmen zum Schutz vor negativen Umwelteinflüssen, kann die Wahrnehmung eines erhöhten Gesundheitsrisikos für Kinder zu Ängsten in der Bevölkerung und insbesondere unter Eltern führen. Durch geeignete Interventionen kann dieses Unsicherheitsgefühl verringert werden, woraus immaterieller Nutzen resultiert.

Somit haben Maßnahmen zum Schutz von Kindergesundheit, welche eigentlich in erster Linie auf die Verringerung physischer Effekte ausgerichtet sind, auch Auswirkungen auf das psychische Wohlbefinden. In Abbildung 1 sind die unterschiedlichen Komponenten des volkswirtschaftlichen Nutzens von Kindergesundheitsprojekten dargestellt.

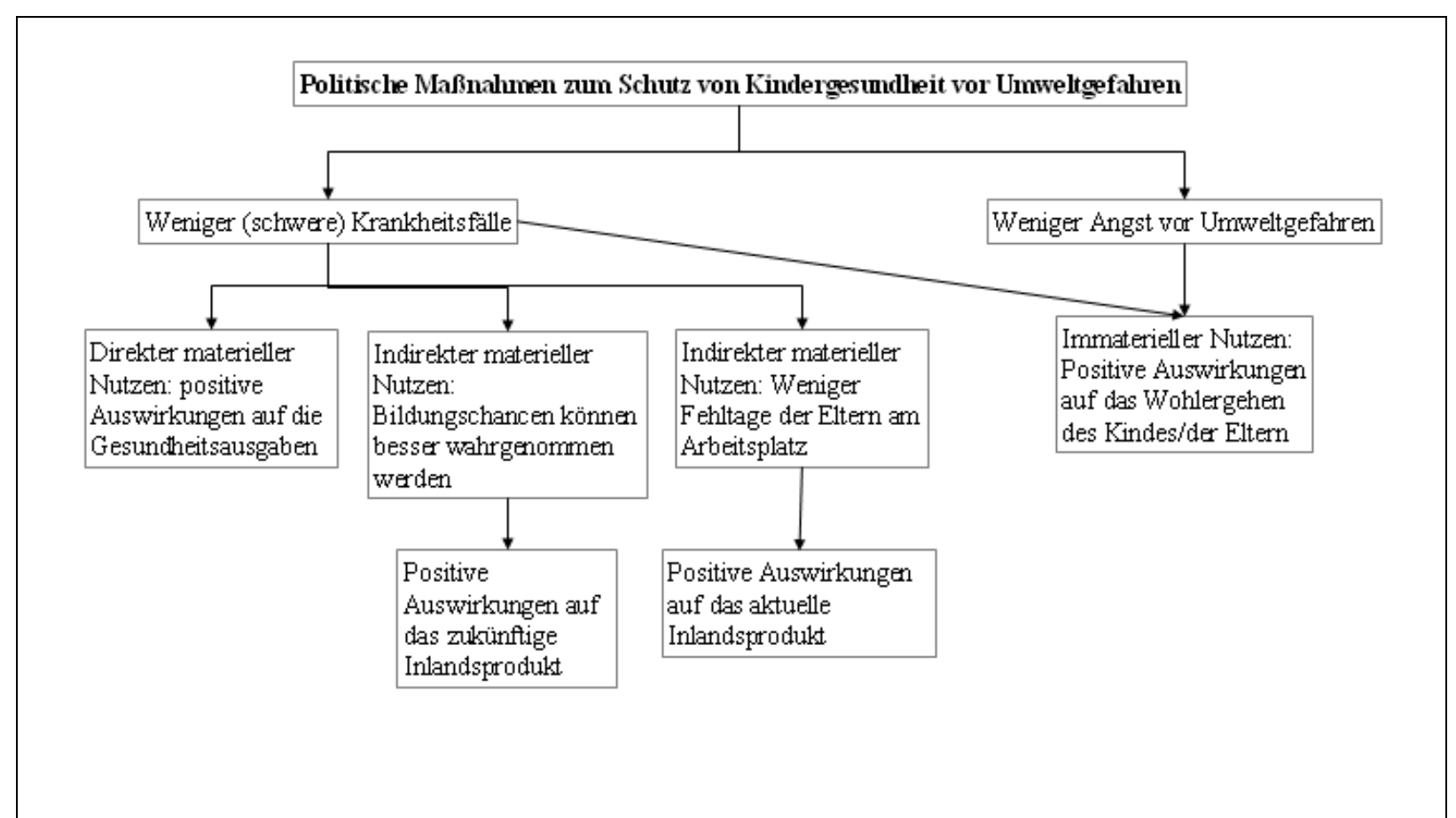

Abbildung 1: Komponenten des volkswirtschaftlichen Nutzens von Kindergesundheitsprojekten (eigene Darstellung)

\section{3 Ökonomische Bewertungskriterien}

Obwohl der Gesundheitsschutz von Kindern hohe Priorität besitzt, können angesichts knapper finanzieller Mittel nicht alle denkbaren umweltpolitischen Maßnahmen durchgeführt werden. 
Die Politik steht somit vor einem Auswahlproblem und benötigt zusätzliche Informationen auf deren Grundlage entschieden wird, ob ein Programm umgesetzt werden soll, oder nicht. Um Fehlinvestitionen in unzweckmäßige Projekte zu verhindern, kann es hilfreich sein, die Wirkungsweise potentiell geeigneter Maßnahmen zu prüfen. Grundsätzlich eignen sich hierzu ökonomische Bewertungsansätze. Diese ermöglichen es, die Auswirkung politischer Maßnahmen ex ante und ex post zu überprüfen.

\section{1 Ökonomische Effizienz}

Zur Beantwortung der Frage, ob die Realisierung einer politischen Maßnahme sinnvoll ist, wird ein geeigneter Maßstab benötigt. Im Fall einer volkswirtschaftlichen Betrachtung wäre dies, die „ökonomische Effizienz“. Eine Maßnahme wird als „ökonomisch effizient“ bezeichnet, wenn ihr volkswirtschaftlicher Nutzen die mit ihr verbundenen volkswirtschaftlichen Kosten übersteigt. Unter dem volkswirtschaftlichen Nutzen versteht man den Wert, den eine bestimmte Projektalternative für die Betroffenen besitzt. Die volkswirtschaftlichen Kosten entsprechen den Opportunitätskosten die entstehen, sollte es zur Umsetzung der Maßnahme kommen. Damit kennzeichnen die volkswirtschaftlichen Kosten einer bestimmten Handlungsalternative den Wert des Verzichts auf die Realisierung.

Hinter dem ökonomischen Effizienzkriterium verbürgt sich das Pareto-Prinzip ${ }^{2}$. Danach ist eine Maßnahme einzuführen, wenn mindestens eine Person besser, aber keine andere schlechter gestellt wird. Weil diesem Konzept in der Realität aber nur schwer entsprochen werden kann, da Entscheidungen für eine Alternative in der Regel mit Gewinner- und Verliererpositionen einhergehen, wurde das so genannte Kompensationskriterium eingeführt ${ }^{3}$. Hiernach dürfen Maßnahmen, bei denen es Bevorteilte und Benachteiligte gibt durchaus umgesetzt werden. Vorraussetzung ist jedoch, dass die Gewinner die Verlierer aus ihrem Zugewinn ausreichend entschädigen können, um das ursprüngliche Nutzenniveau wieder herzustellen. Es kommt dabei nicht darauf an, ob diese Entschädigung tatsächlich vorgenommen wird. Sie muss nur theoretisch möglich sein.

Entsprechend dieser Entscheidungsregel, muss für eine effizienzorientierte Bewertung eines Umweltprojekts geprüft werden, ob die Befürworter der Maßnahme auch dann noch ein höheres Nutzenniveau erreichen, wenn sie die Gegner so weit entschädigen, dass diese sich nach der Umsetzung des Programms auf dem gleichen Nutzenniveau befinden wie davor. Ist

\footnotetext{
${ }^{2}$ Geht zurück auf Vilfredo Pareto, einen Soziologen des 19. Jahrhunderts.

${ }^{3}$ Geht auf Nicholas Kaldor zurück, der das Konzept 1939 entwickelte; John R. Hicks schloss sich ihm später an.
} 
dies der Fall, resultiert aus der Realisierung des Programms eine potentielle ParetoVerbesserung.

Die besondere Bedeutung der ökonomischen Effizienz als Entscheidungshilfe für die Politik lässt sich wie folgt erklären: Wird eine effiziente Maßnahme umgesetzt, ist die Möglichkeit gegeben, dass es keinem Gesellschaftsmitglied schlechter, sondern mindestens einem Teil besser geht als vorher. Schließlich können die Gegner eines Projekts in vollem Umfang aus dem erzielten Nutzen entschädigt werden und deren ursprüngliches Nutzenniveau bleibt erhalten. Der verbleibende Nutzen kommt den Befürwortern der Maßnahme zugute, infolge dessen sich deren Nutzenniveau erhöht. Wird also auch auf Basis von Effizienzüberlegungen über die Umsetzung politischer Programme entschieden, trägt dies dazu bei, das gesellschaftliche Wohlergehen zu steigern. Damit handelt es sich bei der ökonomischen Effizienz um ein normativ begründetes Kriterium. Wird unter dem Gesichtspunkt der Effizienz keine optimale Verteilung der Ressourcen angestrebt, bleiben Potentiale zur Wohlfahrtssteigerung ungenutzt.

Mittels einer Kosten-Nutzen-Analyse kann die ökonomische Effizienz umweltpolitischer Projekte ermittelt werden. Hauptkennzeichen einer Kosten-Nutzen-Analyse ist, dass sämtliche Kosten und der gesamte Nutzen - auch die immateriellen Effekte - einer politischen Maßnahme in Geldeinheiten bewertet werden (SCHÖFFSKI, 2007: 81f). Dadurch werden Kosten und Nutzen eines Projekts in der gleichen Einheit angegeben und können direkt miteinander verglichen werden.

Dies bedeutet, dass der volkswirtschaftliche Nutzen eines umweltpolitischen Programms in Geldeinheiten überführt werden muss. Erreicht werden kann dies, indem die maximale Zahlungsbereitschaft eines Betroffenen für die Umsetzung eines bestimmten umweltpolitischen Vorhabens ermittelt wird. Die Höhe der individuellen Zahlungsbereitschaft ist dann zum einen von den Präferenzen der Person und zum anderen von den Lebensumständen, insbesondere dem Einkommen, abhängig (MARGGRAF, 2005: 65). Je größer das Interesse einer Person an einem umweltpolitischen Vorhaben ist, je mehr wird sie unter Berücksichtigung der persönlichen Einkommenssituation bereit sein, dafür zu zahlen. Auf diese Weise kann der relative Wert eines umweltpolitischen Programms in monetären Größen gemessen werden ${ }^{4}$. Da umweltpolitische Programme aber nicht nur ein einzelnes

\footnotetext{
${ }^{4} \mathrm{Zu}$ beachten ist bei dem Verständnis von volkswirtschaftlichem Nutzen allerdings, dass der Geldbetrag, den eine Person zu zahlen bereit ist lediglich als Vergleichsmaßstab dient, um die Präferenzen nachvollziehbar zu machen. Wenn eine Person fünfzig Euro für die Reduzierung umweltbedingter Gesundheitsrisiken zahlt, heißt das nicht, dass sie einem politischen Gesundheitsprojekt den konkreten Wert eines fünfzig Euro Scheins zuschreibt. Vielmehr vergleicht sie den Wert der Maßnahme mit den anderen Verwendungsmöglichkeiten dieser
} 
Individuum betreffen, sondern gesamtgesellschaftliche Auswirkungen haben, kann der volkswirtschaftliche Nutzen definiert werden als die „Summe der maximale Zahlungsbereitschaften der Projektbefürworter“ (MARGGRAF, 2005: 78ff).

Nun ist es aber denkbar, dass nicht alle Bürger die gleiche umweltpolitische Maßnahme präferieren, da bestimmte Folgen für einige Gesellschaftsmitglieder nachteilig sind. Diese Personen werden keine Zahlungsbereitschaft für die Durchführung der fraglichen Maßnahme haben. Stattdessen werden sie eine Ausgleichszahlung verlangen, um die entstandenen Verluste zu kompensieren. Vor diesem Hintergrund wird unter den volkswirtschaftlichen Kosten einer umweltpolitischen Maßnahme, die „Summe der minimalen Entschädigungsforderungen der Projektgegner“"verstanden (MARGGRAF, 2005: 78ff).

\subsection{Zur Ermittlung von Kosten und Nutzen bei Kindergesundheitsprojekten}

Die empirische Ermittlung der materiellen Kosten- und Nutzenkomponenten erfolgt durch eine Schattenpreisanalyse der relevanten wirtschaftlichen beziehungsweise finanziellen Größen. Dies ist nicht immer einfach, aber mit noch größeren Schwierigkeiten ist die empirische Bestimmung der immateriellen Komponente behaftet. Diese erfolgt mit Hilfe der so genannten Zahlungsbereitschaftsanalyse ${ }^{5}$.

\subsubsection{Vorgehen}

Die Erhebung des immateriellen Nutzens von Kindergesundheitsprojekten muss, wie dargestellt, über die Erfassung der individuellen WTP realisiert werden. Grundsätzlich kommen hierzu zwei Ansätze in Betracht. Entweder es wird versucht, durch Beobachtungen die individuellen Präferenzen aufzudecken, oder man bittet Personen, fiktive Bewertungen für Güter oder für bestimmte Eigenschaften eines Gutes abzugeben (SCHÖFFSKI a, 2007: 372). Bei der ersten Alternative spricht man von „Revealed Preferences Studies“. Der zweite Ansatz wird als „Stated Preferences Studies“ bezeichnet, welche sich wiederum in „Contingent Valuation“ und „Conjoint Analyses“ aufteilen lassen. Während bei der „Contingent Valuation“ das Gut als Ganzes bewertet wird, sollen bei „Conjoint Analyses“ bestimmte Produkteigenschaften betrachtet werden.

Wie bei allen Gesundheitsprojekten lässt sich der Nutzen von politischen Maßnahmen zur Erhaltung von Kindergesundheit nicht an Marktreaktionen ablesen. Schließlich handelt es sich

fünfzig Euro. Eine Zahlungsbereitschaft von fünfzig Euro lässt also darauf schließen, dass einer Person die Reduzierung umweltbedingter Gesundheitsrisiken genauso viel wert ist, wie die beste alternative Verwendung dieser fünfzig Euro (MARGGRAF, 2005: 82f).

${ }^{5}$ Nachstehend bezeichnet der Begriff ,Zahlungsbereitschaftsanalyse“ nur noch die Erhebung der individuellen WTP und dient der Erfassung der immateriellen Komponente. 
bei der Gesundheit um ein Gut, das auf keinem Markt gehandelt wird. Daher muss die Erhebung der WTP für den Schutz von Kindergesundheit über die „Stated Preferences“ Methode erfolgen. Durch die Ermittlung der WTP mithilfe von Befragungen wird auch die Investitionsbereitschaft eines Individuums in die öffentliche Gesundheitsvorsorge berücksichtigt. Mittels der „Revealed Preferences Studies“ lässt sich hingegen nur die WTP für private Maßnahmen zur Vermeidung gesundheitlicher Beeinträchtigen bestimmen (U.S. EPA et al., 2003: 4-2ff). Somit muss die Bestimmung der WTP für die Reduzierung umweltbedingter Gesundheitsrisiken über den „Stated Preferences“- Ansatz erfolgen.

Wird von der „Stated Preferences“- Methode und speziell der „Contingent Valuation“ als Mittel zur Feststellung der individuellen WTP ausgegangen, kann beispielsweise direkt danach gefragt werden, wie viel Geld eine Person bereit wäre für die Reduzierung der Symptome einer bestimmten Krankheit zu zahlen. Auf diese Weise wird der Verbesserung eines Gesundheitszustandes ein bestimmter Geldwert zugeordnet. Denkbar ist auch, die Befragten zu bitten ihre WTP für die Reduzierung eines Gesundheitsrisikos, wie beispielsweise Straßenverkehrslärm oder Luftverschmutzung zu äußern. Der geäußerte Wert stellt dann ein Synonym für den Nutzen dar, den ein Befragter dieser Intervention beimisst.

Besteht ein Rechtsanspruch auf die Reduzierung eines Gesundheitsrisikos, wird diesem aber nicht nachgekommen, muss nach dem Kompensationsbetrag gefragt werden (MARGGRAF, 2005: 71ff). In einem solchen Fall spricht man von der „Willingness to accept“ (WTA), der „Kompensationsforderung“ (MARGGRAF, 2005) oder der „Akzeptanzbereitschaft“ (SCHÖFFSKI a, 2007: 378).

Werden die WTA und die WTP empirisch erhoben, zeigen sich allerdings erhebliche Abweichungen zwischen den Werten (KLOSE, 1999: 110). Die Differenzen zwischen WTP und WTA werden umso größer, je weniger für das zu bewertende Gut ein tatsächlicher Markt vorhanden ist. Die WTA ist dabei zwei- bis fünfmal höher als die individuelle WTP (DUBORG et al., 1994). Handelt es sich bei dem Bewertungsobjekt indes um ein Gut, für das ein echter Marktpreis vorhanden ist, näheren sich WTP und WTA wieder an. Da es sich bei der menschlichen Gesundheit aber um einen Gegenstand handelt, welcher auf keinem Markt gehandelt wird, ist damit zurechnen das WTP und WTA stark differieren. Folglich ist die „Willingness to pay“- Methode prinzipiell dem „Willingness to accept“- Ansatz vorzuziehen, um Überschätzungen des immateriellen Effekts $\mathrm{zu}$ vermeiden, sofern dies nicht im Widerspruch zu formalen Restriktionen steht. 


\subsubsection{Schwierigkeiten bei der Erhebung von Zahlungsbereitschaften}

Die Erhebung der individuellen WTP kann wie oben dargelegt, mittels unterschiedlicher Verfahren erfolgen. Allerdings ist die Ermittlung der WTP in Abhängigkeit von der gewählten Methode mit verschiedenen Problemen behaftet, die die Zuverlässigkeit der Ergebnisse einschränken können ${ }^{6}$.

Es gibt darüber hinaus aber auch noch weitere Nachteile der Zahlungsbereitschaftsanalyse, welche methodenübergreifend von Bedeutung sind. Das schwerwiegendste Problem besteht in der Abhängigkeit der WTP von der persönlichen Einkommenssituation der Befragten. Personen mit höherem Einkommen werden tendenziell auch bereit sein einen höheren Betrag für die Vermeidung eines Gesundheitsrisikos zu zahlen. Dagegen verfügen geringer Verdienende eventuell gar nicht über einen zusätzlich Geldbetrag, den sie für eine Verbesserung einsetzten könnten. Folglich fallen Zahlungsbereitschaft und Zahlungsfähigkeit auseinander (SCHÖFFSKI a, 2008: 381). Die individuelle Bewertung des Nutzens einer bestimmten Alternative erfolgt also immer auch in Abhängigkeit von der finanziellen Situation des Befragten. Inwieweit ein Geldbetrag dann noch als ein akzeptables Maß für den jeweiligen Nutzen eines Gutes angesehen kann, ist fraglich. Eine Entschärfung dieses Problems kann erreicht werden, wenn anstelle eines absoluten Geldbetrags der prozentuale Anteil des Haushalteinkommens für die Verringerung eines Gesundheitsrisikos abgefragt wird (SCHÖFFSKI a, 2008: 382).

Eine weitere Schwierigkeit bei der Erhebung der WTP besteht darin, dass Personen kleine Wahrscheinlichkeiten kaum konsistent bewerten können (BREYER und ZWEIFEL, 1999: 43). BLOMQUIST (1982: 32ff) zeigt zudem anhand verschiedener empirischer Untersuchungen, dass Befragte bei geringfügigen Wahrscheinlichkeitsänderungen (Reduzierung des Krankheitsrisikos von $1 \mathrm{zu}$ einer Million auf $1 \mathrm{zu} 10$ Millionen) kaum zwischen den Alternativen unterscheiden können. Die zuverlässige Zuordnung eines Geldbetrags für eine sehr kleine Änderung der Eintrittswahrscheinlichkeit eines bestimmten Ereignisses ist daher kaum möglich. Gerade bei gesundheitlichen Beeinträchtigungen durch negative Umwelteinflüsse hat man es aber häufiger mit geringen Eintrittwahrscheinlichkeiten für Erkrankungen zu tun. Vor diesem Hintergrund wird weitere Forschung benötigt, um fundierte Aussagen darüber treffen zu können, wie kleine Wahrscheinlichkeiten verständlich kommuniziert werden können.

\footnotetext{
${ }^{6} \mathrm{Zu}$ den genauen methodischen Problemen der einzelnen Erhebungsverfahren sei auf SCHÖFFSKI a (2008: 331ff) verwiesen.
} 


\section{Herausforderungen bei der ökonomischen Bewertung von Kindergesundheit}

Grundsätzlich basieren ökonomische Betrachtungen auf dem Grundsatz der „Konsumentensouveränität“. Der Begriff „Konsumentensouveränität“ bezeichnet die Prämisse, dass jedes Individuum ,sein eigener Richter darüber ist, was ihm gut tut oder schadet“" (BRANDES et al., 2001: 170). Hieraus ergibt sich die Notwendigkeit, Präferenzen für eine Risikoreduktion direkt innerhalb der betroffenen Bevölkerungsgruppe zu erheben.

Der normative Ansatz der „Konsumentensouveränität“ beruht auf der Annahme, dass Personen über die Fähigkeit verfügen, informierte und rationale Entscheidungen zu treffen.

Im Bereich der Medizin oder auch bei Fragen des Verbraucherschutzes hat sich diesbezüglich das Konzept der ,informierten Einwilligung“ (informed consent) durchgesetzt. Zu einer informierten Einwilligung gehören fünf zentrale Elemente: (1) Aufklärung über Alternativen und die dazugehörigen Konsequenzen, (2) Verständnis der bereitgestellten Informationen, (3) Freiwilligkeit, (4) Kompetenz, die Alternativen zu bewerten und gegeneinander abzuwägen, (5) Zustimmung (FADEN und BEAUCHAMP, 1996).

In Bezug auf die Ermittlung von Daten für eine Reduzierung des Gesundheitsrisikos von Kindern ist es fragwürdig, ob eine „informierte Einwilligung“ entsprechend des Grundsatzes der „Konsumentensouveränität“ von den Betroffenen selbst erwartet werden kann. Vielmehr besteht auf wissenschaftlicher Seite die Tendenz, Kinder aufgrund ihres kognitiven Entwicklungstandes als unzuverlässige Entscheidungsträger anzusehen (WORKING PARTY ON National Environmental Policy, 2005: 9). So wird argumentiert, dass Kinder Defizite hinsichtlich relevanter Erfahrungen und kognitiver Verarbeitungsmechanismen besäßen, welche ein eingeschränktes Urteilsvermögen bedingten (HOFFMANN, 2007: 221).

Im Hinblick auf die Erhebung der WTP für eine Reduzierung eines Gesundheitsrisikos kommen zu den oben genannten Bedenken hinsichtlich des Urteilsvermögengens von Kindern zwei weitere Aspekte hinzu. Zum einen sind Kinder den Umgang mit Geld kaum gewohnt. Besonders kleine Kinder haben nur ein begrenztes Verständnis für Tauschgeschäfte, wie beispielsweise zwischen Geld und Gesundheit. Zum anderen verfügen Kinder gar nicht über eigene finanzielle Mittel, welche sie zur Risikoreduktion einsetzen könnten.

Folglich wird es als zielführender angesehen, Kinder nicht direkt zu befragen, sondern die WTP für die Reduzierung eines Gesundheitsrisikos über Stellvertreter zu ermitteln. In der 
Regel sind dies die Eltern oder ein anderer Erziehungsberechtigter (WORKING PARTY ON NATIONAL ENVIRONMENTAL Policy, 2005: 9; SCAPECCHI, 2007: 24) 7 .

Die besondere Eignung der Eltern als Stellvertreter ergibt sich zum einen aus ihrer Nähe zu der Risikogruppe „Kind“. Aufgrund dessen scheinen sie am ehesten in der Lage zu sein, die Präferenzen ihrer Kinder zutreffend wiederzugeben. Zum anderen wird angenommen, dass die Gefahr von Fehleinschätzungen der WTP bei einer Befragung der Eltern geringer ist, als bei Befragungen der Gesamtpopulation. Begründet wird dies damit, dass Eltern von einer Reduzierung des Gesundheitsrisikos ihrer Kinder ebenfalls direkt betroffen seien (WoRKING PARTY ON NATIONAL ENVIRONMENTAL POLICY, 2005: 9, 13).

\subsection{Die Erhebung von Zahlungsbereitschaften über Stellvertreter}

Auch wenn es nahe liegend erscheint, Kinder nicht direkt zu befragen, ist eine Erhebung der Zahlungsbereitschaft über Stellvertreter nicht unproblematisch. Zum einen widerspricht diese Vorgehensweise dem oben dargestellten Grundsatz der „Konsumentensouveränität“. Zum anderen kann es durch die Datenerhebung über Dritte zu Verzerrungen infolge von Altruismus oder Effekten sozialer Erwünschtheit kommen (HOFFMANN, 2007: 231; SCAPECCHI, 2007: 23). Des Weiteren führt diese mittelbare Feststellung der Zahlungsbereitschaftswerte dazu, dass der Prozess der familiären Entscheidungsstrukturen berücksichtigt werden muss (WORKING PARTY ON NATIONAL EnVIRONMENTAL Policy, 2005: 11ff). Nachstehend werden diese spezifischen Bewertungsprobleme jeweils detaillierter erläutert.

\subsection{Verzerrungen durch Altruismus}

Ganz allgemein wird unter dem Begriff „Altruismus“ die Sorge um das Wohlergehen anderer verstanden. Altruistische Handlungen müssen nicht zwingend auf idealistischen oder normativen Einstellungen beruhen, sondern können auch durch eine besondere emotionale Nähe begründet sein. Ein Beispiel dafür wäre die Mutter-Kind-Beziehung.

Ökonomen unterscheiden zwischen zwei Formen des Altruismus: dem „elterlichen“ und dem „,nicht-elterlichem Altruismus“. „Elterlicher Altruismus“ resultiert aus den Bemühungen um das Wohlergehen des Kindes unabhängig von dessen eigentlichen Präferenzen. Somit

\footnotetext{
${ }^{7}$ Als weitere Perspektive, aus denen die Bewertung von Kindergesundheit erfolgen kann, kommen die soziale Perspektive und die Erwachsene-als-Kinder Perspektive in Betracht. Bei der sozialen Perspektive werden alle erwachsenen Mitglieder einer Gesellschaft nach ihrer WTP für die Reduzierung eines Gesundheitsrisikos für Kinder befragt. Bei der Kinder-als-Erwachsene Perspektive werden die befragten Personen gebeten, sich in ihre Kindheit zurück zu versetzen und rückblickend ihr „kindliche“ WTP anzugeben, allerdings unter Einbeziehung ihrer Fähigkeit rationale Entscheidungen treffen zu können.
} 
umschreibt „elterlicher Altruismus“ die Handlungsgrundlage dafür, was Eltern glauben, was gut für ein Kind sei. Ein klassisches Beispiel für eine Verhaltensweise, welche durch „elterlichen Altruismus“ motiviert ist, ist die folgende Aussage: „Iss deinen Spinat, es interessiert mich nicht, ob du ihn magst oder nicht. Spinat ist gut für Dich“ (HofFMANN, 2007: 218f).

Hingegen bezeichnet „nicht-elterlicher Altruismus“ das Interesse der Eltern an den Wünschen des Kindes. Beispiele hierfür sind alle Handlungen, die dazu dienen, das Kind zufrieden zu stellen. Bezogen auf die Ernährung kann dies die Zubereitung der Lieblingsspeise des Kindes sein unabhängig davon, ob Eltern diese für gesund halten oder nicht.

Während „elterlicher Altruismus“ also eher die Präferenzen der Eltern widerspiegelt, werden durch „nicht- elterlichen Altruismus“ die Wünsche des Kindes erfasst.

Es ist davon auszugehen, dass die Erhebung von Zahlungsbereitschaften von Eltern für eine Risikoreduktion des Kindes durch beide Formen des Altruismus beeinflusst wird. Jedoch besteht in der Wissenschaft Uneinigkeit darüber, inwieweit diese Beeinflussung zu Verzerrungen der Werte führt. Zum Teil wird unterstellt, dass der Einfluss von „elterlichem“ und „,nicht-elterlichem Altruismus“ Doppelzählungen bedinge, da der Nutzen einer Intervention zweimal berücksichtigt werde: einmal in der Nutzenfunktion des Kindes und ein weiteres Mal in der Nutzenfunktion der Eltern (BERGSTROM, 1982: 16ff). Dies führe schließlich zu einer Überschätzung des Nutzens, beziehungsweise zu einer Verzerrung der Zahlungsbereitschaft nach oben. Vor diesem Hintergrund gab es in der Vergangenheit Versuche, den Einfluss von „nicht-elterlichem Altruismus“ zu isolieren und aus ökonomischen Nutzenbetrachtungen auszuschließen (HOFFMANN, 2007: 217f).

In einem anderen Teil der Literatur wird das Argument der Doppelzählungen damit entkräftet, dass eine Reduzierung des Gesundheitsrisikos von Kindern im Grunde auch beide Nutzenfunktionen beeinflusse. Die der Eltern, indem sie beispielsweise weniger Angst vor einer Erkrankung ihres Kindes haben müssten. Und die des Kindes, indem es effektiv weniger gefährdet sei. Bedenkt man, dass die elterlichen Präferenzen und damit auch ihre Nutzenfunktion eher durch den ,elterlichen Altruismus“ repräsentiert werden, während sich die Interessen der Kinder in ,nicht-elterlichem Altruismus“ ausdrücken, dürfte der oben dargelegten Argumentation von Doppelzählungen nicht zugestimmt werden. Vielmehr sei es sogar notwendig beide Formen des Altruismus in eine Betrachtung mit einzubeziehen. $\mathrm{Zu}$ Doppelzählungen käme es nur dann, wenn die Präferenzen der Kinder zusätzlich erhoben würden (HOFFMANN, 2007: 230). 
Die Gefahr von Doppelzählungen und Überschätzungen der Zahlungsbereitschaft steigt jedoch generell, wenn nicht nur Eltern, sondern die gesamte Bevölkerung befragt wird. Aufgrund generell altruistischer Haltungen gegenüber Kindern kann es dazu kommen, dass der Nutzen eines Kindes mehrmals berücksichtigt wird, obwohl die Auswirkungen einer Intervention tatsächlich nur einmal zur Beeinflussung der Nutzenfunktion dieses Kindes beitragen. Dementsprechend geht man davon aus, dass die gesamtgesellschaftliche Zahlungsbereitschaft für die Reduzierung des Gesundheitsrisikos von Kindern höher ist, als die elterliche (U.S. EPA, 2009: 14).

Ein Lösungsansatz bezüglich der Messung des wohlfahrtsökonomischen Nutzens einer Reduzierung des Gesundheitsrisikos von Kindern wird darin gesehen, die elterliche Zahlungsbereitschaft als unterste Grenze eines bestimmten Intervalls anzusehen, in dem sich der tatsächliche Wert bewegt. Wird die Zahlungsbereitschaft der übrigen (erwachsenen) Gesellschaftsmitglieder dazu addiert, könnte dies als die oberste Grenze dieses Intervalls verstanden werden (HoFFMANN, 2007: 231).

\subsection{Verzerrungen infolge von Effekten sozialer Erwünschtheit}

Der Begriff „sozialer Erwünschtheit“ bezeichnet eine Fehlerquelle im Rahmen sozialwissenschaftlicher Befragungen. Die befragten Personen beantworten Fragen dabei nicht entsprechend ihrer persönlichen Meinung, sondern geben Antworten von denen sie glauben, dass diese gesellschaftlich akzeptiert - oder anders gesagt „sozial erwünscht“ sein. Dieser Effekt tritt meist bei Fragen auf, die Werte und Normen der Gesellschaft berühren.

Grundsätzlich können zwei Arten sozialer Erwünschtheit unterschieden werden: „,kulturelle soziale Erwünschtheit“ und „situative soziale Erwünschtheit“. „Kulturelle soziale Erwünschtheit“" beruht auf internalisierten allgemeinen Verhaltenserwartungen z. B. aufgrund traditioneller Geschlechterrollen. Dagegen resultiert „situative soziale Erwünschtheit“ aus konkreten Stimuli in der Befragungssituation, wie zum Beispiel der Hautfarbe des Interviewers (ESSER, 1986: 317; SCHNELL et al., 1999: 331f). Gemein ist beiden Formen „sozialer Erwünschtheit“, dass sie zu Antwortverzerrungen und damit zu Einschränkungen der Reliabilität der erhobenen Daten führen. Vor diesem Hintergrund gehört es zu den allgemeinen methodischen Herausforderungen sozialwissenschaftlicher Untersuchungen, Befragungen so zu gestalten, dass die Teilnehmer ihre wahren Einstellungen offen legen und ihre Antworten nicht anhand sozial erwünschter Kriterien ,editieren“.

Im Hinblick auf die Erhebung einer Zahlungsbereitschaft für eine Reduzierung des Gesundheitsrisikos von Kindern ist besonders mit Effekten „kultureller sozialer 
Erwünschtheit“"zu rechen. Diese könnten aus der gesellschaftlichen Erwartung resultieren, dass Eltern das Wohlergehen eines Kindes über das eigene stellen sollten. Dieser Anspruch kann dazu führen, dass Eltern die Zahlungsbereitschaft für die Reduzierung des Gesundheitsrisikos des Kindes aufgrund der wahrgenommen moralischen Verpflichtung grundsätzlich höher angegeben, als für die Reduzierung des eigenen Gesundheitsrisikos (Working PARTY ON NATiONAL ENVIRONMENTAL Policy, 2005: 9). Verringert werden kann dieser Effekt, indem die Zahlungsbereitschaften für die Reduzierung des eigenen Gesundheitsrisikos und die des Kindes getrennt voneinander, in separaten Befragungen, erhoben werden.

\subsection{Die Berücksichtung familiärer Entscheidungsprozesse}

Wird die Zahlungsbereitschaft für die Reduzierung des Gesundheitsrisikos von Kindern nicht direkt von den Betroffenen selbst, sondern über deren Eltern erhoben, verschiebt sich der Bewertungskontext von der individuellen auf eine haushaltsbezogene Ebene. Dieser Umstand erschwert die Messung der Zahlungsbereitschaft. Es geht dann nicht mehr ausschließlich um die Erfassung individueller Einstellungen, sondern es müssen kollektive (familiäre) Einstellungs- und Handlungszusammenhänge in die Betrachtung einbezogen werden (Working PARTY ON NATIONAL ENVIRONMENTAl POLICY, 2005: 11).

Diese notwendige Berücksichtigung familiärer Entscheidungsstrukturen kann über die Integration eines privaten Haushaltsmodells erfolgen. Diese Modelle beschreiben die Beziehungen innerhalb eines Haushalts und sollen darüber hinaus die Regeln der Ressourcenvereilung zwischen den Mitgliedern offen legen. Grundsätzlich unterscheidet man in der Ökonomie zwischen zwei Arten von privaten Haushaltsmodellen: dem (1) „Modell gemeinsamer Präferenzen“ (Unitary Model) und dem (2) „Kooperativen Haushaltsmodell“ (Collective Model).

Im Rahmen des „Unitary Model“ werden die finanziellen Ressourcen (Income pooling) und auch die Entscheidungskompetenzen eines Haushalt zu einer Einheit zusammengefasst, wobei Kinder eine passive und Eltern eine aktive Position einnehmen. Das „Unitary Model“ legt eine einheitliche Nutzenfunktion für die gesamte Familie zugrunde. Die meisten ökonomischen Studien zur Bewertung von Kindergesundheit gehen von diesem einheitlichen Ansatz aus (DICKIE und GERKING, 2006: 124).

Das „Collective Model“ berücksichtigt dagegen individuelle Unterschiede hinsichtlich der Einstellungen und Präferenzen einzelner Haushaltsmitglieder. Es geht nicht von grundsätzlichen Übereinstimmungen zwischen den Individuen aus, stattdessen bilden die 
einzelnen Meinungen eine gemeinschaftliche Entscheidungsgrundlage. Die Entscheidungen eines Haushalts sind dann das Ergebnis kooperativer oder auch nicht-kooperativer Verhandlungsprozesse (WORKING PARTY ON NATIONAL ENVIRONMENTAL POLICY, 2005: 12). Das „Collective Model“ unterstellt somit individuelle Nutzenfunktionen für jedes einzelne Familienmitglied.

Weiterhin ist im „Collective Models“ auch implizit die Möglichkeit enthalten, dass einzelne Haushaltsmitglieder spezifische Verantwortungsbereiche besitzen, innerhalb derer ihnen die alleinige Entscheidungskompetenz obliegt.

Damit unterscheiden sich beide Ansätze vor allem dadurch, dass das „Collective Model“ die einzelnen Familienmitglieder als Individuen betrachtet, wodurch auch Kinder $\mathrm{zu}$ unabhängigen Entscheidungsträgern mit eigenen Nutzenfunktionen werden. Hingegen wird die Familie im „Unitary Model“ als eine Einheit verstanden, welche nach Maximierung einer einzigen gemeinsamen Nutzenfunktion strebt.

In Anbetracht der Existenz verschiedener Haushaltsmodelle stellt sich die Frage, welcher Ansatz die Charakteristik familiärer Entscheidungen zutreffend beschreiben kann.

Diese Frage kann nicht allgemeingültig beantwortet werden. Die Wahl des passenden Haushaltsmodells wird stark vom jeweiligen Untersuchungsgegenstand beeinflusst. Ist eine Familie kollektiv und in gleicher Weise von einem Ereignis betroffen, kann das „Unitary Model“ durchaus die geeignete Basis für die Untersuchung familiärer Interaktion darstellen. Können die Auswirkungen eines bestimmten Ereignisses jedoch durch individuelles Abwehrverhalten gemindert werden, oder sind einzelne Haushaltsmitglieder stärker betroffen als andere, stellt das „Unitary Model“ nicht mehr die adäquate Betrachtungsgrundlage dar. Schließlich werden die Nutzenfunktionen der Familienmitglieder durch ein solches Ereignis verschieden stark beeinflusst, was dazu führt, dass auch die darauf folgenden Reaktionen unterschiedlich ausfallen werden. Das gleiche gilt, wenn die Nutzenfunktion eines Haushaltsmitglieds durch das Verhalten eines anderen beeinflusst wird. So ist es denkbar, dass bestimmte Handlungen zur Maximierung der Nutzenfunktion eines Familienmitglieds beitragen, während die Nutzenfunktion eines anderen Haushaltsmitglieds dadurch negativ beeinflusst wird (Working Party on National EnVIRONMENTAl Policy, 2005: 12f). Ein Beispiel hierfür wäre das Rauchen in Anwesenheit von Kindern.

Somit zeigt sich, dass die Präferenzen und Nutzenwerte innerhalb einer Familie keinesfalls immer einheitlich sind. Folglich können auch die Zahlungsbereitschaften für die Reduzierung eines Risikos innerhalb eines Haushalts differieren. Wird dann trotzdem von dem „Unitary 
Model“" ausgegangen und der Wert eines Familienmitglieds als gültiger Wert für den gesamten Haushalt angenommen, kommt es zu Messfehlern. Für die Qualität der erhobenen Daten ist die Wahl des zutreffenden Haushaltsmodells daher von entscheidender Bedeutung.

In der Praxis empirischer Forschung verschwimmt oft die Unterscheidung zwischen individuellen und haushaltsbezogenen Bewertungszusammenhängen (BATEMAN und MUNRO, 2006: 1). Im Rahmen von Untersuchungen zur Ermittlung der Zahlungsbereitschaft mittels der „Stated Preferences“ Methode wird meist nicht berücksichtigt, ob die dargelegten Präferenzen auf individuellen Nutzenabwägungen oder Überlegungen des gesamten Haushalts beruhen. Vielmehr wird häufig pauschal davon ausgegangen, dass Deckungsgleichheit zwischen den Werten, die von einem Haushaltsmitglied erhoben werden und den Werten des Gesamthaushalts besteht. Dementsprechend wird implizit, meist ohne nähere Prüfung der Richtigkeit dieser Annahme, das „Unitary Model“ unterstellt.

Im Rahmen der aktuellen Forschung wird diese Vorgehensweise jedoch zunehmend kritisch diskutiert. BATEMAN und MUNRO (2006) untersuchten, inwieweit sich die individuell erhobene Zahlungsbereitschaft von dem kollektiven Wert eines Zwei-Personen-Haushalts quantitativ unterscheidet. Dazu führten sie ein Experiment durch, bei dem die Zahlungsbereitschaft zusammenlebender Paare für fettreduzierte und für pestizidfreie Lebensmittel ermittelt wurde. Es wurden insgesamt 121 verheiratete und unverheiratete Paare befragt. Die teilnehmenden Paare wurden per Zufallsauswahl in zwei Gruppen aufgeteilt. In der ersten Gruppe wurden beide Partner zusammen interviewt und gebeten, gemeinsam die Auswahlentscheidungen $\mathrm{zu}$ treffen (Treatment 1). Auf diese Weise wurde eine haushaltsbezogene Zahlungsbereitschaft für beide Produktattribute erhoben. Aus den Paaren der zweiten Gruppe wurde jeweils ein Partner zufällig ausgewählt, welcher allein die Bewertungsaufgabe vornahm (Treatment 2).

Es zeigt sich, dass die gemeinschaftlich erhobenen Zahlungsbereitschaftswerte signifikant von den individuellen Werten abweichen (BATEMAN und Munro, 2006: 16ff). Folgt man dem „Unitary Model“" müsste die im Einzelinterview ermittelte Zahlungsbereitschaft (Treatment 2) jedoch den kollektiven Werten entsprechen. Somit liefern BATEMAN und MunRo (2006) einen empirischen Beweis für die Unangemessenheit einer generellen Unterstellung des „Unitary Model“. Folglich dürfen individuell erhobene Zahlungsbereitschaften in haushaltsbezogenen Untersuchungszusammenhängen nicht einfach mit dem kollektiven Wert des Gesamthaushalts gleichgesetzt werden. Andernfalls kann es zu erheblichen Messfehlern bei der Erhebung der haushaltsbezogenen Zahlungsbereitschaften kommen. 
Auch SCAPECCHI (2006) entkräftet die allgemeine Gültigkeit des „Unitary Models“. Legt man diesen Ansatz zugrunde, sollte die Zahlungsbereitschaft beider Partner für den gleichen Bewertungsgegenstand identisch sein. SCAPECCHI (2006) weist jedoch darauf hin, dass Frauen hinsichtlich der Gesundheit ihrer Kinder risikoaverser verhalten als Männer. Daher seien auch die Zahlungsbereitschaften der Mütter für eine Reduzierung des Gesundheitsrisikos ihrer Kinder höher als die der Väter.

Soll die Zahlungsbereitschaft eines Haushalts zuverlässig erhoben werden, reicht es also nicht aus, eine zufällig ausgewählte Person dieses Haushalts zu befragen. Alle Haushaltsmitglieder im Rahmen einer Untersuchung zu interviewen, ist in der Praxis jedoch oft nicht möglich. Als Alternative zur zufälligen Auswahl schlagen daher BATEMAN und MUNRO (2006) vor, einen Verantwortlichen für den Bewertungsgegenstand mittels verschiedener Kontrollfragen zu identifizieren. Dieser soll dann anstelle des zufällig ausgewählten Haushaltsmitglieds befragt werden. Wird so vorgegangen, verringert sich die Differenz zwischen den im Gruppeninterview erhobenen Zahlungsbereitschaften und den individuellen Werten. Trotzdem, so stellen BATEMAN und MUNRO (2006) im Rahmen der statistischen Analyse fest, verschwindet dieser Effekt nicht. Die Unterschiede zwischen individueller und haushaltsbezogener Zahlungsbereitschaft bleiben nach wie vor signifikant (BATEMAN und MunRO, 2006: 16ff).

\section{$5 \quad$ Aktuelle Entwicklung in der Umwelt- und Gesundheitspolitik}

In den letzten Jahren ist die Durchführung von Wirtschaftlichkeitsuntersuchungen zur Bewertung umweltpolitischer Projekte in Anbetracht knapper finanzieller Ressourcen immer populärer geworden. Speziell in Bezug auf den Schutz von Kindergesundheit wird im Rahmen umweltpolitischer Programme immer häufiger die Durchführung von Wirtschaftlichkeitsuntersuchungen gefordert. So ist es ein wesentliches Ziel der „Europäischen Strategie für Umwelt und Gesundheit 2004-2010“ die Forschungsgrundlage zur Durchführung ökonomischer Bewertungen, insbesondere im Bereich „Umwelt und Kindergesundheit“" zu verbessern. Exemplarisch für das verstärkte Interesse an ökonomischen Bewertungsmethoden ist auch das dreijährige Projekt der OECD "Valuation of EnvironmentRelated Health Impacts with a particular Focus on Children“ (VERHI 2006-2008) oder das Projekt "Economic Valuation of transport-related health effects" (PEP 2003-2007) der WHO. In den USA ist die Durchführung von Wirtschaftlichkeitsuntersuchungen in Verbindung mit umweltpolitischen Interventionen zur Reduzierung von Gesundheitsgefahren für Kinder seit dem Jahr 1997 sogar gesetzlich vorgeschrieben (Executive Order (E.O.) 13045). Dort wird 
verstärkt auf die Kosten-Nutzen-Analyse als ökonomisches Bewertungsinstrument zurückgegriffen (U.S. EPA et al., 2003). Auch in Großbritannien und den skandinavischen Ländern werden immer häufiger Kosten-Nutzen-Analysen im Gesundheitssektor zur Realisierung ökonomischer Evaluationen eingesetzt (SCHÖFFSKI, 2007: 82).

Vor dem Hintergrund der internationalen Entwicklung ist davon auszugehen, dass ökonomische Bewertungsansätze und speziell die Kosten-Nutzen-Analysen im Zusammenhang mit umweltpolitischen Maßnahmen zum Schutz von Kindergesundheit zukünftig auch in Deutschland vermehrt zur Anwendung kommen werden. Folglich muss es darum gehen, bestehende Probleme bei der Durchführung der Kosten-Nutzen-Analyse zu lösen, um ein zuverlässiges Instrument $\mathrm{zu}$ politischen Entscheidungsunterstützung bereitzustellen.

Obwohl die Durchführung von Kosten-Nutzen-Analysen in vielen Bereichen der Umweltund Gesundheitspolitik Europas noch nicht obligatorisch ist, konnte in den letzen Jahren ein steigendes Interesse an ökonomischen Bewertungsansätzen zur Entscheidungsunterstützung verzeichnet werden. Vor diesem Hintergrund gibt es Bestrebungen standardisierte Modelle für die Anwendung in der Umwelt- und Gesundheitspolitik zu entwickeln. Durch eine Formulierung einheitlicher Leitlinien soll die Durchführung von Wirtschaftlichkeitsuntersuchungen erleichtert und verbessert werden. Außerdem ist es Ziel die Einbeziehung ökonomischer Kriterien in politische Entscheidungsprozesse voranzutreiben.

In den folgenden Ausführungen werden zwei Ansätze beschrieben, auf deren Grundlage eine ökonomische Bewertung umweltpolitischer Gesundheitsprojekte erfolgen kann. Es handelt sich dabei um Beiträge, die explizit die besondere Rolle von Kindern berücksichtigen. Zuerst wird in Kapitel 6 auf ein europäisches Modell zur ökonomischen Bewertung verkehrsbedingter Erkrankungen und dem dahinter stehenden umweltpolitischen Programm eingegangen. Anschließend wird in Kapitel 7 ein Bewertungsansatz zur Beurteilung der Effizienz umweltpolitischer Maßnahen zum Schutz von Kindergesundheit in den USA vorgestellt.

\section{6 "Pan-European Programme on Transport, Health and Environment"}

Im Rahmen der dritten Ministerkonferenz „Umwelt und Gesundheit“ der WHO wurde im Jahr 1999 die „Charta für Verkehr, Umwelt und Gesundheit“ verabschiedet, aus der 2002 das "Transport, Health and Environment Pan-European Program ” (PEP) entstanden ist. Dieses Vorhaben hatte das Ziel, umwelt- und gesundheitsbezogene Aspekte in die Verkehrspolitik 
von Europa zu integrieren. Unter anderem sollte ein Modell erarbeitet werden, mit dem verkehrsbedingte Erkrankungen ökonomisch bewertet werden können. Besonderer Wert wurde dabei auf die Berücksichtigung von Kindern und deren spezifische Risikosituation gelegt. Im Folgenden wird auf das PEP und die Entwicklung eines Bewertungsleitfadens näher eingegangen.

\subsection{Entwicklung und grundlegende Inhalte}

Im Kontext des PEP wurde ein Ansatz zur ökonomischen Bewertung verkehrsbedingter Gesundheitseffekte mit einem besonderen Fokus auf Kinder entwickelt. In die Betrachtung einbezogen wurden ausschließlich die gesundheitsschädlichen Auswirkungen des Straßenverkehrs. Die besondere Berücksichtigung von Kindern begründet sich mit einer erhöhten Anfälligkeit für verkehrsspezifische Gefahrenquellen und Umweltkontaminationen. Im Jahr 2007 wurde ein Nachfolgeprogramm des PEP initiiert mit dem Titel: „Review of methods and development of guidance for the economic valuation of transport-related health effects, with a particular focus on children“(PEPa). Ziel war es, den im PEP entwickelten Bewertungsansatz mithilfe neu erschienener wissenschaftlicher Literatur zu aktualisieren und zu verbessern. Dazu wurde eine Vielzahl epidemiologischer Arbeiten sowie neuer Studien zur ökonomischen Bewertung menschlicher Gesundheit aufgearbeitet. Von besonderem Interesse waren die Unterschiede zwischen Kindern und Erwachsenen. Es wurde zum einen der Frage nachgegangen, ob das Risiko sowie die Art und Schwere der gesundheitlichen Beeinträchtigungen zwischen Kindern und Erwachsen differieren. Zum anderen wurde geprüft, ob zwischenzeitlich neue methodische Ansätze entwickelt worden sind, welche zu einer Verbesserung der monetären Bewertung umweltbedingter Gesundheitsprobleme in der Praxis beitragen können (BOESCH et al., 2008: 17ff).

Der Focus des PEPa lag erneut ausschließlich auf den gesundheitsschädlichen Auswirkungen des Straßenverkehrs in Europa. Die in der Untersuchung berücksichtigten Gesundheitsprobleme resultierten aus verschiedenen verkehrsspezifischen Quellen. Dazu gehören Luftverschmutzung, Straßenverkehrslärm, Verkehrsunfälle und mangelnde körperliche Aktivität. Berücksichtigt wurden neben direkten gesundheitlichen Konsequenzen auch weniger offensichtliche Folgen, wie soziale Isolation und verminderte Lebensqualität in Gegenden mit besonders hohem Verkehraufkommen (BOESCH et al., 2008: 9). 


\subsection{Zur Ermittlung verkehrsbedingter Krankheitskosten}

Der überarbeitete Bewertungsleitfaden des PEPa liefert eine Anleitung zur praktischen Durchführung einer ökonomischen Betrachtung verkehrsbedingter Erkrankungen. Mithilfe eines vierstufigen Ansatzes können die gesamten Auswirkungen des Straßenverkehrs auf die menschliche Gesundheit quantifiziert werden.

Grundsätzlich erfolgt die ökonomische Bewertung der Kosten für umweltbedingte Erkrankungen durch Straßenverkehrslärm, Luftverschmutzung und Verkehrsunfälle nach dem gleichen System (BOESCH et al., 2008: 26ff). Für die gesundheitlichen Auswirkungen unzureichender körperlicher Aktivität als Folge des motorisierten Straßenverkehrs kann noch kein abschließendes Modell konstruiert werden. Hierfür fehlten zum Zeitpunkt der Projektdurchführung die entsprechenden Daten. Es ist zwar nachgewiesen, dass die Nutzung privater oder öffentlicher Verkehrsmittel immer häufig dem Fahrrad fahren oder zu Fuß gehen vorgezogen wird. Dazu, wie sich diese Entwicklung qualitativ und quantitativ auf den Gesundheitszustand einer Gesellschaft auswirkt, waren jedoch noch keine zuverlässigen Ergebnisse verfügbar (BOESCH et al., 2008: 65ff). Aus diesem Grund ist das nachstehend beschriebene Modell zur Ermittlung der Krankheitskosten vorbehaltlos nur für die ökonomische Bewertung verkehrsbedingter Gesundheitseffekte durch Straßenverkehrslärm, Luftverschmutzung und Verkehrsunfälle einsetzbar.

\subsubsection{Die Kostenkalkulation entsprechend des PEPa}

Die Ermittlung verkehrsbedingter Krankheitskosten erfolgt prinzipiell in vier Schritten. Diese sind in Tabelle 1 dargestellt und werden anschließend ausführlich erläutert.

\begin{tabular}{|l|l|}
\hline Schritt 1 & $\begin{array}{l}\text { Festlegung der Verkehrsform und Identifikation der dazugehörigen } \\
\text { Umwelteffekte }\end{array}$ \\
\hline Schritt 2 & $\begin{array}{l}\text { Schätzung der Emissionsstärke und des Expositionsgrades der } \\
\text { betrachteten Population }\end{array}$ \\
\hline Schritt 3 & Erstellung einer Expositions-Reaktions-Funktion \\
\hline Schritt 4 & Monetarisierung der ermittelten Gesundheitseffekte \\
\hline
\end{tabular}

Tabelle 1: Grundsätzliches Vorgehen zur Ermittlung umweltbedingter Krankheitskosten (BOESCH et al., 2008: 26ff).

Zunächst ist in einem ersten Schritt festzulegen, für welche Verkehrsform (Straßen- oder Schienenverkehr) die entstandenen Krankheitskosten kalkuliert werden sollen. Basierend auf 
dieser Entscheidung sind die dazu gehörigen Fahrzeugtypen (PKW, Busse etc.) und die darauf zurückzuführenden Umwelteffekte (Luftverschmutzung, Verkehrsunfälle etc.) zu identifizieren.

In einem zweiten Schritt sind das Ausmaß der Emissionen, sowie die Stärke der Exposition für die Bevölkerung zu ermitteln. Diese Informationen können für Verkehrsunfälle aus den entsprechenden Opferstatistiken entnommen werden. Die Belastungen durch Straßenverkehrslärm und Luftverschmutzung können mithilfe von Emissionstabellen für jede Verkehrsart und jeden Fahrzeugtyp kalkuliert werden.

Im Rahmen des dritten Schrittes ist eine Expositions-Reaktions-Funktion für eine ausgewählte Population zu erstellen. Die Konstruktion der Expositions-Reaktions-Funktion erfolgt prinzipiell durch die Kombination des Expositionsgrades mit der Anzahl resultierender Krankheits- oder Todesfälle. Die Bestimmung belastungstypischer Gesundheitseffekte kann unter Bezugnahme epidemiologischer Studien umgesetzt werden. Mithilfe dieser ExpositionsReaktions-Funktion können Aussagen darüber getroffen werden, inwiefern eine spezifische Gefahrenquelle das Vorkommen einer bestimmten Erkrankung innerhalb der untersuchten Population beeinflusst (BOESCH et al., 2008: 27).

Die Erstellung einer Expositions-Reaktions-Funktion kann auf unterschiedliche Art erfolgen. In Betracht kommt die Life-table Methode, die vereinfacht dargestellt auf einem Vergleich zwischen einer bestimmten Grundsituation (im Hinblick auf eine spezifische Belastung) und einer Veränderung dieser Situation (durch Beseitigung der Belastung) beruht. Der Effekt einer spezifischen Exposition ergibt sich dann aus der Differenz der Todes- oder Krankheitsfälle zwischen den beiden Szenarien ${ }^{8}$.

In einem letzten Schritt (Schritt 4) werden die ermittelten Gesundheitseffekte ökonomisch bewertet. Hierbei werden einerseits die direkten und indirekten materiellen Kosten jeder einzelnen verkehrsbedingten Erkrankung berechnet. Dazu zählen beispielsweise Behandlungs- und Verwaltungskosten sowie Produktionsverluste infolge der gesundheitlichen Beeinträchtigungen der Bevölkerung. Anderseits sind aber auch die immateriellen Kosten zu bewerten, welche durch Schmerzen oder eine allgemein verminderte Lebensqualität verursacht werden können. Somit setzen sich die Gesamtkosten verkehrsbedingter Erkrankungen aus drei unterschiedlichen Kostenarten zusammen. Aus der Summe aller Kosten ergeben sich spezifische Kennzahlen für die einzelnen verkehrsbedingten

\footnotetext{
${ }^{8}$ Zur genauen Vorgehensweise bei der Erstellung einer Expositions-Reaktions-Funktion siehe BOESCH et al. (2008: .27).
} 
Erkrankungen. Diese Kennzahlen können dann entweder die Kosten für jeden einzelnen Fall einer bestimmten Krankheit angeben, oder sie können sich auf die gesamte Fallzahl beziehen. Die Bestimmung der materiellen Kosten erfolgt entsprechend des PEPa Ansatzes durch die Ermittlung aller finanziellen Aufwendungen und Einbußen, die durch verkehrsbedingte Gesundheitsprobleme entstehen. Diese können unter Verwendung verschiedener Ansätze errechnet werden. Hierzu zählen die „Krankheitskosten“ oder englisch „Costs-of-Illness“ (COI), der „Wert des statistischen Lebens“ in englisch „Value of Statistical Life“ (VSL), und die „Kosten für verlorene Lebensjahre“ beziehungsweise die englische Bezeichnung „Value of Life Years lost“ (VLYL). Welche Methode letztlich die zur Kostenberechung geeignete ist, hängt von der Art der zu erwartenden Gesundheitseffekte (tödlich oder nicht tödlich) ab. Prinzipiell werden der Krankheitskostenansatz für nicht tödliche Erkrankungen und die „Kosten für verlorene Lebensjahre“ für tödliche Ausgänge empfohlen (BOESCH et al., 2008: 10).

Zur Feststellung der immateriellen Kosten wird die Zahlungsbereitschaftsanalyse vorgeschlagen. Durch die Erhebung der WTP wird auch die Einbeziehung von Ausgaben für Medikamente oder individuelle privatfinanzierte Präventionsmaßnahmen erreicht.

Zur endgültigen Berechnung der gesamten verkehrsbedingten Krankheitskosten müssen die jeweiligen Kosten aller auf den Verkehr zurückzuführenden Krankheiten aufsummiert werden (BOESCH et al., 2008: 10). Wurden die Kosten einer Krankheit pro Fall erhoben, müssen zunächst alle Krankheitsfälle infolge der verschiedenen Gefahrenquellen addiert und mit den entsprechenden monetären Kennzahlen multipliziert werden (BOESCH et al., 2008: 10).

Die erforderlichen Daten zur Berechnung der verkehrsbedingten Krankheitskosten können im Idealfall aus aktuellen epidemiologischen oder ökonomischen Studien übernommen werden.

Schließlich gilt für jeden der vier beschriebenen Schritte, dass fehlende Daten für ein bestimmtes Untersuchungsgebiet durch entsprechende Werte aus vergleichbaren Regionen ersetzt werden können. Eine solche Übertragung von Werten stellt jedoch grundsätzlich nur die zweitbeste Alternative dar, weil hierdurch die Genauigkeit der Ergebnisse beeinträchtigt werden kann. Darüber hinaus sollten alle notwendigen Daten zur Berechnung der verkehrsbedingten Krankheitskosten möglichst zeitgleich erhoben werden, damit die einzelnen Werte kompatibel sind und zu einem Gesamtergebnis zusammengefasst werden können (BOESCH et al., 2008: 72).

Zur Prüfung der Genauigkeit der ermittelten verkehrsbedingten Krankheitskosten wird die Durchführung eines statistischen Sensitivtests empfohlen (BOESCH et al., 2008: 32, 34). Am sichersten kann dies mit einer Monte Carlo Simulation erfolgen (BOESCH et al., 2008: 32), 
einem wahrscheinlichkeitstheoretischen Verfahren zur quantitativen Risikoschätzung ${ }^{9}$. Mittels dieses Tests kann für unsichere Parameter das 95\%-Konfidenzintervall bestimmt werden, welches den Bereich kennzeichnet, in dem mit 95\%-iger Wahrscheinlichkeit der wahre Wert zu finden ist. Die errechneten Krankheitskosten können dann mit diesem Intervall abgeglichen werden. Basierend auf diesem Vergleich können Aussagen darüber getroffen werden, wie genau die ermittelten Werte die tatsächliche Kostenhöhe abbilden können.

\subsection{Herausforderungen bei der Monetarisierung verkehrsbedingter}

\section{Erkrankungen}

Während der Entwicklung des Leitfadens zur Berechnung verkehrsbedingter Gesundheitskosten im Rahmen des PEPa wurden auch Grenzen bei der ökonomischen Bewertung verkehrsbedingter Erkrankungen deutlich. Diese beziehen sich auf die Schritte zwei bis vier des Bewertungsschemas. Hinsichtlich der Schritte zwei und drei resultieren Probleme aus einem Mangel an Daten sowie Erkenntnissen zum Zusammenspiel von verkehrsbedingten Umweltrisiken und den entstehenden Gesundheitseffekten, vor allem in Bezug auf Kinder (BoEsCH et al., 2008: 42, 47). Bei Schritt vier erschweren methodische Defizite die Messung der krankheitsbedingten Kosten. Diese methodischen Schwierigkeiten beziehen sich zum einen auf die monetäre Bewertbarkeit bestimmter Gesundheitseffekte, wie beispielsweise psychosozialer Folgen. Zum anderen bestehen Bewertungsprobleme bei bestimmten Bevölkerungsgruppen. Auch hier zeigen sich Schwierigkeiten insbesondere bei der zutreffenden Berücksichtigung von Kindern, da bei ihnen aufgrund der Differenzen hinsichtlich der Häufigkeit und Schwere gesundheitlicher Beeinträchtigungen oft andere Werte als bei Erwachsenen zugrunde gelegt werden müssen (BOESCH et al., 2008: 9, 23).

Aus den genannten Bewertungsschwierigkeiten ergeben sich verschiedene Unsicherheiten, welche die Genauigkeit der Ergebnisse einschränken können. Dadurch kann es sowohl zu Über- als auch zu Unterschätzungen der verkehrsbedingten Krankheitskosten kommen. Nachstehend werden diese Unsicherheitsfaktoren und deren Einfluss auf die Zuverlässigkeit der Ergebnisse genauer beschrieben und kommentiert.

\subsubsection{Schritt 2: Dokumentationslücken}

Eine Verzerrung der tatsächlichen verkehrsbedingten Krankheitskosten kann auch aufgrund einer unvollständigen statistischen Erfassung der gesundheitlichen Beeinträchtigungen erfolgen. Gerade bei vergleichsweise harmlosen Verkehrsunfällen, werden leichte

\footnotetext{
${ }^{9}$ Zur Durchführung und Interpretation von Monte Carlo Simulationen siehe Ecoplan, Infras (2008).
} 
Verletzungen häufig nicht polizeilich gemeldet und entsprechend registriert. Daher ist gerade in diesem Bereich mit einer hohen Dunkelziffer $\mathrm{zu}$ rechnen. Um erheblichen Unterschätzungen der entstehenden Krankheitskosten entgegenzuwirken, muss das tatsächliche Ausmaß der Verkehrsunfälle mit Personenschaden geschätzt werden (BOESCH et al., 2008: 36). Allerdings sind auch diese Schätzungen oft nur Annährungen an den wahren Wert und dadurch ebenfalls mit Fehlern belastet. Werden die Kosten auf der Basis dieser Daten kalkuliert, sind sowohl Über- als auch Unterschätzungen des tatsächlichen Werts nicht auszuschließen.

\subsubsection{Schritt 3: Unsicherheiten aufgrund fehlender Daten}

Ist nicht ausreichend untersucht, ob und inwieweit eine bestimmte Erkrankung auf eine verkehrsspezifische Belastung zurückzuführen ist, kann trotz eines vermuteten kausalen Zusammenhangs keine Expositions-Reaktion-Funktion erstellt werden. Die Gesundheitseffekte, bei denen der Entstehungshintergrund nicht eindeutig geklärt ist, werden also nicht in die Kalkulation der Krankheitskosten einbezogen, obwohl anzunehmen ist, dass diese durch eine verkehrsspezifische Gefahrenquelle ausgelöst werden. Diese Vorgehensweise stellt zwar sicher, dass nur Gesundheitseffekte ökonomisch bewertet werden, bei denen ein bewiesener Zusammenhang zwischen auslösender Umweltkontamination und resultierender Erkrankung besteht. Andererseits bedingt die Vernachlässigung noch nicht abschließend untersuchter Wirkungsbeziehungen vermutlich eine Unterschätzung der tatsächlichen Kosten (BOESCH et al., 2008: 63).

Aus Unklarheiten hinsichtlich der Entstehungszusammenhänge bestimmter verkehrsbedingter Erkrankungen können sich aber auch Verzerrungen in entgegengesetzter Richtung ergeben. Im Fall von Überschneidungen in den Wirkungszusammenhängen kann es zu einer Überschätzung der verkehrsbedingten Krankheitskosten kommen. So sind bestimmte Gesundheitseffekte nicht immer eindeutig einer spezifischen Gefahrenquelle zuzuordnen. Beispielsweise können Herzkreislauferkrankungen sowohl durch verkehrsbedingte Luftverschmutzung als auch durch Straßenverkehrslärm sowie der häufig auftretenden Kombination aus beidem ausgelöst werden. Folglich ist es notwendig festzustellen, auf welche Exposition ein Gesundheitseffekt zurückzuführen ist, beziehungsweise zu welchen Anteilen ein gesundheitliches Problem durch eine bestimmte Exposition verursacht wird. Geschieht dies nicht, sondern wird die Erkrankung einer Person in vollem Umfang beiden Belastungsquellen zugeordnet, kommt es zu Doppelzählungen und infolgedessen zu einer Überschätzung der verkehrsbedingten Krankheitskosten. 
Der derzeitige Wissensstand ermöglicht jedoch nicht immer die anteilige Aufschlüsselung der Ursache-Wirkungs-Beziehungen. Im Fall einer Herzkreislauferkrankung ist es beispielsweise nicht möglich anzugeben, zu welchem Anteil diese auf Luftverschmutzung oder Straßenverkehrslärm beruht. Dieses Defizit stellt eine mögliche Fehlerquelle bei der Ermittlung der Krankheitskosten dar und sollte daher Gegenstand zukünftiger Forschung sein (BoESCH et al., 2008: 71f).

Weitere Überschätzungen der tatsächlichen verkehrsbedingten Krankheitskosten können entstehen, wenn der nivellierende Effekt von Ausgleichs- oder Ersatzhandlungen nicht bedacht wird. So ist es zwar nachgewiesen, dass die Nutzung von privaten PKWs oder öffentlichen Verkehrsmitteln immer häufiger dem Fahrrad fahren und dem zu Fuß gehen vorgezogen wird. Bedacht wird dabei aber häufig nicht, dass allein die Tatsache, dass weniger Fahrrad gefahren oder zu Fuß gegangen wird, nicht automatisch auch zu einem Rückgang der körperlichen Aktivität im Ganzen führt. Es ist zum Beispiel vorstellbar, dass zwar weniger Fahrrad gefahren, dafür aber immer häufiger gejoggt wird. Das Joggen stellte in diesem Fall ein Substitut für das Fahrrad fahren dar, welches den Einfluss einer geringeren Fahrradmobilität auf die körperliche Aktivität insgesamt ausgleichen kann (BOESCH et al., 2008: 64).

\subsubsection{Schritt 3: Die Berücksichtigung von Kindern}

$\mathrm{Zu}$ Verzerrungen der ermittelten Krankheitskosten kann es auch kommen, wenn Kinder als besonders gefährdete Risikogruppe bei der Kalkulation wie Erwachsene behandelt werden. Sind keine epidemiologischen Daten speziell für Kinder verfügbar, muss die gleiche Expositions-Reaktions-Funktion zugrunde gelegt werden, die auch für Erwachsenen verwendet wird. Die Tatsache, dass Kinder eine erhöhte Anfälligkeit für verkehrsbedingte Erkrankungen besitzen und sich ihre gesundheitlichen Beeinträchtigungen hinsichtlich Art und Schwere von denen eines Erwachsen unterscheiden können, wird damit ignoriert. Ein Beispiel hierfür stellt die Ermittlung der Krankheitskosten durch verkehrsbedingten Lärm dar. Obwohl es in der epidemiologischen Literatur Hinweise gibt, dass Lärmbelästigungen die Entwicklung von Kindern negativ beeinflussen, kann noch keine spezifische ExpositionsReaktions-Funktion erstellt werden. Dementsprechend kann auch die besondere Gefährdungslage von Kindern im Rahmen der ökonomischen Bewertung nicht angemessen berücksichtigt werden (BOESCH et al., 2008: 47). Aus der Vernachlässigung des erhöhten Gefährdungsgrades von Kindern, kann eine Unterschätzung der tatsächlichen Krankheitskosten resultieren. 
Ähnliches gilt, wenn die besondere gesundheitliche Situation von Kindern nur für eine spezielle Altersgruppe hinreichend untersucht ist. Im Bereich der Luftverschmutzung beispielsweise stehen genug Informationen für Kinder bis zu einem Jahr zur Verfügung, um eine spezifische Expositions-Reaktions-Funktion konstruieren $\mathrm{zu}$ können. Somit ist es möglich, die Krankheitskosten infolge verkehrsbedingter Luftverschmutzungen für diese Altersgruppe gesondert zu berechnen (BOESCH et al., 2008: 58ff). Jedoch fehlt für Kinder und junge Erwachsene bis zum dreißigsten Lebensjahr die notwendige Datengrundlage, um auch für sie eine eigene Expositions-Reaktions-Funktionen erstellen zu können. Daher wurden Personen zwischen einem und neunundzwanzig Jahren aus der Ermittlung der Krankheitskosten durch Luftverschmutzungen ausgeschlossen. Durch diese Konzentration auf Altersgruppen, für die die Ursache-Wirkungs-Beziehungen eindeutig geklärt sind, ist jedoch mit einer Unterschätzung der wahren Kosten zu rechnen (BOESCH et al., 2008: 63).

\subsubsection{Schritt 4: Probleme bei der Bewertbarkeit bestimmter Gesundheitseffekte}

Zur Kalkulation der gesamten verkehrsbedingten Krankheitskosten sind nicht nur die entstehenden materiellen Aufwendungen $\mathrm{zu}$ berechnen, sondern es müssen auch die immateriellen Kosten der Betroffenen bewertet werden. Die Ermittlung der immateriellen Kosten, welche beispielsweise durch verminderte Lebensqualität infolge chronischer Erkrankungen entstehen können, erfolgt im Rahmen des PEPa durch die Erhebung der individuellen WTP. Die Einbeziehung von Zahlungsbereitschaftswerten ist jedoch mit verschiedenen Schwierigkeiten behaftet. Zum einen variiert die WTP für die Reduzierung eines spezifischen Risikos in Abhängigkeit von der Erhebungsmethode beziehungsweise der jeweiligen Interviewsituation. Zum anderen beeinflussen Alter und Einkommen der Befragten die individuelle Zahlungsbereitschaft.

Schließlich ist die WTP von dem zu bewertenden Risiko und dem resultierenden Gesundheitseffekt abhängig. Verschiedene Studien belegen, dass sich die Werte für die Reduzierung eines akuten Sterberisikos deutlich von denen eines vorzeitigen Todes infolge von Langzeitexpositionen unterscheiden (BOESCH et al., 2008: 32). Dieser Sachverhalt ist vor allem deshalb problematisch, da bestimmte Gefahrenquellen wie die Luftverschmutzung sowohl zu einem plötzlichen Versterben als auch zu einem schleichenden Tod führen können (BoEsCH et al., 2008: 56).

Zusammenfassend lässt sich schlussfolgern, dass beispielsweise nicht einfach eine generelle Zahlungsbereitschaft für die Verringerung des Straßenverkehrslärms zugrunde gelegt werden dürfte. Vielmehr müssten die WTP für alle möglichen gesundheitlichen Beeinträchtigungen 
jeweils einzeln abgefragt werden. Auf diese Weise erhielte man spezifische Werte für jeden $\mathrm{zu}$ erwartenden Gesundheitseffekt einer bestimmten verkehrsbedingten Gefahrenquelle. Um Überschätzungen der wahren Zahlungsbereitschaft auszuschließen, wird empfohlen für die ökonomische Bewertung der verkehrsbedingten Gesundheitseffekte durch Luftverschmutzung entweder ausschließlich die kurz- oder die langfristigen Folgen in die Betrachtung einzubeziehen (BOESCH et al., 2008: 54). Dieser Ansatz kann jedoch nicht als zufrieden stellend angesehen werden, da die Ausklammerung bestimmter Gesundheitseffekte wiederum eine Unterschätzung der tatsächlichen WTP zur Folge haben kann.

\subsubsection{Schritt 4: Latenz}

Bestimmte gesundheitliche Folgen verkehrsspezifischer Umweltkontaminationen treten nicht ummittelbar auf, sondern werden erst einige Jahre später offensichtlich. Besonders bei verkehrsbedingten Luftverschmutzungen ist mit einer solchen verzögerten Wirkung zu rechnen. Aufgrund dessen führt eine Verbesserung der Luftqualität nicht umgehend auch zu einem Rückgang der belastungstypischen Erkrankungen. Diese Latenzzeit zwischen Exposition und Reaktion des menschlichen Körpers muss bei der Kalkulation der Krankheitskosten beachtet werden (BOESCH et al., 2008: 57). Dazu müssen die ermittelten Kosten für zukünftige Gesundheitseffekte diskontiert werden. Hierfür wurde beispielsweise für die Schweiz eine Diskontierungsrate von $1 \%$ pro Jahr zugrunde gelegt ${ }^{10}$. Die kalkulierten Kosten für die Langezeiteffekte verkehrsbedingter Luftverschmutzungen sind dann entsprechend zu korrigieren (BOESCH et al., 2008: 61).

Aber auch wenn die verzögerte Wirkung bestimmter Belastungen bei der Ermittlung der Krankheitskosten berücksichtigt wird, kann die Latenz zu Ungenauigkeiten führen. Die zukünftig steigende Lebenserwartung der Bevölkerung erhöht die Anzahl der verlorenen Lebensjahre. Damit steigen auch die Kosten für einen vorzeitigen Tod infolge einer Erkrankung durch verkehrsbedingte Luftverschmutzungen. Diesem Effekt kann durch die Diskontierung nicht Rechnung getragen werden kann. Folglich ist eine Unterschätzung der Krankheitskosten sehr wahrscheinlich (BOESCH et al., 2008: 63).

\subsubsection{Schritt 4: Die Berücksichtigung von Kindern}

Prinzipiell ist das ökonomische Bewertungsmodell des PEPa auf Erwachsene und Kinder gleichermaßen anwendbar. Die Berechnung der kinderspezifischen materiellen Kosten erfolgt im Rahmen des PEPa Ansatzes durch die Berücksichtigung der höheren Anzahl verbleibender

\footnotetext{
${ }^{10}$ Zur Berechnung der Diskontierungsrate siehe BOESCH et al. (2008: 61).
} 
Lebensjahre (BOESCH et al., 2008: 37). Die vergleichsweise höhere Lebenserwartung von Kindern beeinflusst die Anzahl entgangener Lebensjahre infolge verkehrsbedingter Gesundheitseffekte. Da jedem Lebensjahr mittels des Value of Life Years Lost (VLYL) ein bestimmter monetärer Wert zugeordnet werden kann, ergeben sich für Kinder höhere Kosten als für Erwachsene. Dies muss bei der Kalkulation berücksichtigt werden, um Unterschätzungen der Krankheitskosten entgegenzuwirken.

Hinzu kommt, dass es in der Literatur zahlreiche Hinweise auf die Alterabhängigkeit des Werts des statistischen Lebens (VSL) gibt. So belegen verschiedene Studien für das Leben eines Kindes einen höheren oder mindestens identischen Wert, wie für das eines Erwachsenen (BlomQuist, 2003; MounT et. al., 2001). Dagegen zeigen andere Untersuchungen, dass der VSL eines Erwachsenen den eines Kindes deutlich übertrifft (JENKINS et al., 2001). ALBERINI und CHIABAI (2006) gehen indessen von einem individuellen VSL aus, wobei sie tendenziell für jüngere Personen höhere Werte nachweisen als für Ältere.

Die Ermittlung der kinderspezifischen immateriellen Kosten verkehrsbedingter Gesundheitsschäden stellt eine weitere Herausforderung dar. Es müsste hierzu die individuelle Zahlungsbereitschaften der Betroffenen erhoben werden. Gegenwärtig gibt es jedoch, wie angesprochen, keine Methode mit der die WTP von Kindern zuverlässig gemessen werden kann (BOESCH et al., 2008: 23). Daher werden für Kinder momentan noch die gleichen Werte wie für Erwachsene angenommen, um die Krankheitskosten zumindest annährend ermitteln zu können (BOESCH et al., 2008: 33, 47).

Es gibt allerdings empirische Befunde, die darauf hindeuten, dass die Zahlungsbereitschaft für die Reduzierung eines Gesundheitsrisikos in Abhängigkeit vom Alter der Betroffenen variiert. Die Ergebnisse zur Bestimmung der Richtung des Zusammenhangs sind jedoch, ähnlich wie die des VSL, sehr heterogen. Während einige Autoren von einer negativen Beziehung zwischen dem Alter und der individuellen WTP ausgehen (HAMMITT und GRAHAM, 1999; LIU et al., 2000; DiCKIE und GERKING, 2006), weisen andere Studien einen Anstieg der WTP bis zu einem bestimmten Alter nach (JOHANNESSON und JOHANSSON, 1996; JOHANNESSON und JOHANSSON, 1997).

Auch wenn bisher nicht abschließend geklärt ist, wie die WTP und der VSL durch das Alter beeinflusst werden, ist jedoch sicher, dass ein Zusammenhang besteht. Werden für Kinder trotz der Alterunterschiede pauschal die gleichen Werte wie für Erwachsene zugrunde gelegt, kann es zu erheblichen Verzerrungen der Gesamtkosten kommen. 


\subsection{Abschließende Bemerkung zum PEPa}

Der Bewertungsleitfaden des PEPa ist ein brauchbarer Ansatz zur Ermittlung der Kostenkomponente, da neben den direkten und indirekten Kosten auch die immateriellen Effekte berücksichtigt werden. Damit entspricht das PEPa den Empfehlungen der gesundheitsökonomischen Literatur ${ }^{11}$.

Jedoch weist die im PEPa beschriebene Vorgehensweise zur Kostenberechnung verschiedene Schwachpunkte auf. So kann es bei der praktischen Durchführung ökonomischer Analysen entsprechend des im PEPa dargestellten Ansatzes zu Schwierigkeiten kommen. Diese können einerseits aus methodischen Problemen bei der ökonomischen Bewertung des Gutes „Gesundheit“ resultieren. Anderseits kann aber auch das Fehlen relevanter epidemiologischer Daten, Probleme bei der Kostenkalkulation bedingen. Besonders die Ermittlung der verkehrbedingten Krankheitskosten von Kindern ist noch mit vielen Unsicherheiten behaftet sowohl im medizinischen als auch im ökonomischen Bereich. Für die spezifischen Bewertungsprobleme im Zusammenhang mit der Berechnung kinderspezifischer Kosten kann letztlich auch das PEPa keine innovativen Lösungsansätze bieten. So kann der Rückgriff auf WTP-Werte oder die Expositions-Reaktions-Funktion eines Erwachsenen zur Berechnung der verkehrsbedingten Krankheitskosten von Kindern, aufgrund der identifizierten Risikounterschiede, keine befriedigende Lösung darstellen. Um die Genauigkeit der ermittelten Werte und damit die Qualität der Endergebnisse zu verbessern, wird weitere Forschungsarbeit zur WTP, zum VSL und VLYL für Kinder aber auch auf medizinischer Seite notwendig sein.

Darüber hinaus ist es in Bezug auf die Bestimmung der immateriellen Kosten fraglich, ob die Zahlungsbereitschaftsanalyse überhaupt die angemessene Erfassungsmethode darstellt. Schließlich räumt Artikel 24 Abs.1 der Kinderrechtskonvention jedem Kind das Recht auf ein „erreichbares Höchstmaß an Gesundheit“ ein. Auch in der „Europäischen Charta Umwelt und Gesundheit“ wird jedem Menschen der Anspruch auf eine „Umwelt, die ein höchstmögliches $\mathrm{Ma}$ an Gesundheit und Wohlbefinden ermöglicht" zugestanden. Vor diesem Hintergrund wäre zu prüfen, ob die immateriellen Kosten nicht über die Kompensationsforderung für eine verkehrsbedingte Gesundheitsgefährdung ermittelt werden müssten. Diese generelle Fragestellung wird im PEPa jedoch ausgeklammert. Da die Kompensationsforderung in der Regel höher liegt als die WTP, kann die Erfassung der immateriellen Kosten mittels der Zahlungsbereitschaftsanalyse zu Unterschätzungen der verkehrbedingten Krankheitskosten führen.

\footnotetext{
${ }^{11}$ Siehe hierzu GREINER (2007: 49ff)
} 
Positiv hervorzuheben ist indes, dass im PEPa zahlreiche Schwierigkeiten offen gelegt werden, welche mit der ökonomischen Bewertung von Kindergesundheit verbunden sind. Diese bieten einen Anhaltspunkt für zukünftige Forschungsbemühungen im Bereich Umweltökonomie und Kindergesundheit.

\section{7 "Children's Health Valuation Handbook"}

Das amerikanische "Children's Health Valuation Handbook" ist ein weiterer Beitrag zur Umsetzung ökonomischer Bewertungen von Kindergesundheit. In den USA ist die Durchführung von Wirtschaftlichkeitsuntersuchungen für den Bereich „Umwelt und Kindergesundheit“" bereits seit dem Ende der 1990er Jahre gesetzlich verankert und damit fester Bestandteil der politischen Entscheidungsfindung. Die Executive Order (E.O.) 13045 „Protection of Children from Environmental Health Risks and Safety Risks“ fordert unter anderem die Bewertung neuer umweltpolitischer Maßnahmen bezüglich ihrer ökonomischen Effizienz und des Nutzens für die Gesundheit von Kindern.

Vor diesem Hintergrund hat die „National Agenda to Protect Children’s Health from Environmental Threats“ die U.S. Environmental Protection Agency (U.S. EPA) im Jahr 2000 den „Guidelines for Preparing Economic Analyses“ entwickelt. Dieses Dokument stellt ein Leitfaden zur Durchführung allgemeiner ökonomischer Bewertungen politischer Programme dar. Ergänzend dazu ist das "Children's Health Valuation Handbook” entstanden, welches die ökonomische Bewertung von Kindergesundheit und umweltbedingten Gesundheitsrisiken explizit thematisiert. Die spezifischen Herausforderungen, die mit der ökonomischen Bewertung von Kindergesundheit verbunden sind, werden im „Guidelines for Preparing Economic Analyses“ nicht in vollem Umfang berücksichtigt. Daher ist erweiternd das "Children's Health Valuation Handbook" formuliert worden. Beide Dokumente sollen von Analytikern kombiniert genutzt werden, um neue umweltpolitische Maßnahmen hinsichtlich ihrer Auswirkungen auf die Gesundheit von Kindern zu beurteilen ${ }^{12}$.

\subsection{Entwicklung und grundlegende Inhalte}

Das „Children's Health Valuation Handbook“ wurde von dem "National Center for Environmental Economics" in Zusammenarbeit mit dem "Office of Children's Health Protection" im Jahr 2003 entwickelt. Das Handbook bezieht sich auf die Realisierung von Kosten-Nutzen-Analysen zur Bewertung umweltpolitischer Maßnahmen zum Schutz von

\footnotetext{
${ }^{12}$ In den folgenden Ausführungen wird nur das „Children`s Health Valuation Handbook“ vorgestellt, da es im Rahmen dieses Beitrags nicht um eine generelle Diskussion ökonomischer Bewertungsansätze geht, sondern gezielt auf die Bewertung von Kindergesundheit eingegangen wird.
} 
Kindergesundheit (U.S. EPA et al., 2003: 1-1). Es hat im Vergleich zum PEPa jedoch eher einen informativen Charakter und stellt weniger eine konkrete Durchführungsanweisung zur Umsetzung von Kosten-Nutzen-Analysen dar. Darüber hinaus steht im „Children's Health Valuation Handbook“ insbesondere die Bestimmung des Nutzen umweltpolitischer Maßnahmen zum Schutz von Kindergesundheit im Mittelpunkt. Diese Nutzenermittlung soll entsprechend des Handbooks über die Erhebung von WTP und ausnahmsweise über COI erfolgen (U.S. EPA et al., 2003: 2-5; 4-1; U.S. EPA, 2000: 60f). Zur Effizienzbeurteilung werden dem geschätzten Nutzen die zu erwartenden Kosten einer umweltpolitischen Maßnahme gegenübergestellt.

Das Handbook stellt ausschließlich die ökonomische Bewertung von zeitnahen Gesundheitsgefahren für Kinder in den Vordergrund und bezieht sich nicht auf die Risiken für zukünftige Generationen (U.S. EPA et al., 2003: 1-2). Weiterhin zielt das „Children’s Health Valuation Handbook“ auf die ex ante Bewertung von Gesundheitseffekten beziehungsweise deren Vermeidung ab. Diese ex ante Bewertung ist der ex post Betrachtung grundsätzlich vorzuziehen, wenn die Effizienz als Umsetzungsvoraussetzung für politische Maßnahmen gilt (U.S. EPA et al., 2003: 2-5).

\subsection{Vorgehen bei der Nutzenerfassung von Kindergesundheitsprojekten}

Die Evaluierung des Nutzens eines umweltpolitischen Kindergesundheitsprojekts umfasst nach Vorgabe des Handbooks sechs einzelne Schritte (U.S. EPA et al., 2003: 1-4). Diese werden in der Tabelle 2 dargestellt. Während die ersten vier Schritte in den Zuständigkeitsbereich von Risikobewertern fallen, ist die Umsetzung der beiden letztgenannten Aufgabe der Ökonomen. Darüber hinaus werden im „Children’s Health Valuation Handbook“ die spezifischen Herausforderungen der ökonomischen Bewertung politischer Programme zum Schutz von Kindergesundheit aufgezeigt. Dabei stehen die Aspekte, welche im Zusammenhang mit den Schritten fünf und sechs der grundsätzlichen Bewertungsschemas zu erwarten sind, im Vordergrund. 


\begin{tabular}{|c|c|}
\hline Arbeitsschritte & Ziele \\
\hline $\begin{array}{l}\text { 1. Identifizierung schädlicher } \\
\text { Gesundheitseffekte }\end{array}$ & $\begin{array}{l}\text { Zusammenstellung der möglichen gesundheitlichen } \\
\text { Beeinträchtigungen, die bei Kindern aufgrund des zu } \\
\text { bewerten Umweltproblems zu erwarten sind }\end{array}$ \\
\hline $\begin{array}{l}\text { 2. Feststellung der Dosis- } \\
\text { Wirkungs-Beziehungen }\end{array}$ & $\begin{array}{l}\text { Beschreibung der Wahrscheinlichkeit und des Ausmaßes } \\
\text { gesundheitlicher Beeinträchtigungen in Abhängigkeit von } \\
\text { der Belastungsstärke }\end{array}$ \\
\hline $\begin{array}{l}\text { 3. Abschätzung der } \\
\text { Expositionsstärke }\end{array}$ & $\begin{array}{l}\text { Messung oder Schätzung der Belastung von Kindern durch } \\
\text { Umweltkontaminationen hinsichtlich des Ausmaßes, der } \\
\text { Häufigkeit, der Dauer sowie der Verteilung innerhalb der } \\
\text { Population }\end{array}$ \\
\hline 4. Risikocharakterisierung & $\begin{array}{l}\text { Abschätzung der Wahrscheinlichkeit, dass negative } \\
\text { Gesundheitseffekte bei Kindern aufgrund der betrachteten } \\
\text { Umweltgefahr auftreten können. Hierzu gehört auch eine } \\
\text { Erläuterung der statistischen und medizinischen } \\
\text { Unsicherheiten, welche mit der Schätzung verbunden sein } \\
\text { können }\end{array}$ \\
\hline $\begin{array}{l}\text { 5. } \begin{array}{l}\text { Bestimmung der } \\
\text { Wohlfahrtseffekte }\end{array}\end{array}$ & $\begin{array}{l}\text { Präzisierung, welche Auswirkungen die Änderungen des } \\
\text { Gesundheitsstatus eines Kindes auf die Wohlfahrt hat. } \\
\text { Relevante Größen sind hier beispielsweise Einschränkungen } \\
\text { der Lebensqualität durch Schmerzen, Ausgaben für } \\
\text { medizinische Behandlungen und Fehltagen der Kinder in der } \\
\text { Schule }\end{array}$ \\
\hline $\begin{array}{l}\text { 6. } \text { Monetarisierung der } \\
\text { Wohlfahrtseffekte }\end{array}$ & $\begin{array}{l}\text { Monetarisierung der } \mathrm{zu} \text { erwartenden Wohlfahrtseffekte } \\
\text { mittels geeigneter ökonomischer Methoden }\end{array}$ \\
\hline
\end{tabular}

Tabelle 2: Ermittlung des Nutzens von umweltpolitischen Gesundheitsprojekten (U.S. EPA et al., 2003: 14)

\subsection{Risikounterschiede zwischen Kindern und Erwachsenen}

Bedeutsam für die Bestimmung von Wohlfahrtseffekten sind Risikounterschiede zwischen Kindern und Erwachsenen. Die Identifizierung potentieller Risikounterschiede betrifft die „Risikocharakterisierung“ (Schritt 4) und fällt häufig in das Forschungsgebiet der Medizin. 
Da im „Children’s Health Valuation Handbook“ die Monetarisierung von Kindergesundheit betrachtet wird, werden diese Risikounterschiede nur kurz thematisiert.

Grundsätzlich ist jedoch zu beachten, dass viele Umwelteinflüsse für Kinder nicht die gleiche Gefahr darstellen wie für Erwachsene ${ }^{13}$. Allerdings liegen spezielle toxikologische Daten für Kinder häufig nicht vor. Eine Ausnahme stellen nur die zahlreichen epidemiologischen Untersuchungen zu den Auswirkungen erhöhter Luftverschmutzung auf die Gesundheit von Kindern dar. Sind solche altersspezifische Daten verfügbar, wird grundsätzlich deren Verwendung empfohlen (U.S. EPA et al., 2003: 2-3). In allen anderen Fällen müssen Ergebnisse aus erwachsenenbezogenen Studien oder auch aus Tierversuchen übernommen und näherungsweise an die neue Population angepasst werden. Es ist jedoch davon auszugehen, dass der Mangel an alterspezifischen epidemiologischen Daten bei der Abschätzung des konkreten Risikos für Kinder zu Ungenauigkeiten führt. Folglich sind Aussagen zu Wahrscheinlichkeiten möglicher gesundheitlicher Beeinträchtigungen meist mit Unsicherheiten behaftet.

\subsection{Ermittlung des Nutzens über die Zahlungsbereitschaft}

Zur Ermittlung von Wohlfahrtseffekten messen Ökonomen den Nutzen eines Projekts auf Basis der WTP. Im „Children’s Health Valuation Handbook“ werden unterschiedliche Erhebungsmethoden zur Ermittlung kinderspezifischer WTP vorgestellt und deren Potential zur Messung des Nutzen von Kindergesundheitsprojekten diskutiert (U.S. EPA et al., 2003: 41ff). Die Erhebungsverfahren lassen sich in direkte und indirekte Methoden gliedern. Zu den indirekten Ansätzen gehören die „Hedonic Method“ und der „Averting Behavior Approach“. Die „Stated Preferences Method“ beschreibt hingegen eine direkte Methode zur Bestimmung der WTP. In Tabelle 4 werden die genannten Verfahren überblickartig dargestellt.

\footnotetext{
${ }^{13}$ Wie es zu diesen Risikounterschieden im Einzelnen kommt, wurde in Kapitel 2.2 bereits erläutert.
} 


\begin{tabular}{|c|c|}
\hline Methode & Vorgehen \\
\hline $\begin{array}{l}\text { Hedonic Method (Hedonischer } \\
\text { Ansatz) }\end{array}$ & $\begin{array}{l}\text { Über Eigentumswerte, die mit dem Schutz } \\
\text { Kindergesundheit in Verbindung stehen } \\
\text { Lohndifferenzen, wird auf die WTP geschlossen }\end{array}$ \\
\hline $\begin{array}{l}\text { Averting Behavior Approach } \\
\text { (Vermeidungskostenansatz) }\end{array}$ & $\begin{array}{lcccr}\text { Über die Realisierung } & \text { von } & \text { Abwehrreaktionen } & \text { (z.B. } \\
\text { Kaufentscheidungen) } & \text { zur } & \text { Reduzierung } & \text { von } \\
\text { Gesundheitsgefahren } & \text { wird } & \text { die } \quad \text { WTP } & \text { für } \\
\text { Kindergesundheit abgeleitet } & & & \end{array}$ \\
\hline $\begin{array}{l}\text { Stated Preferences } \\
\text { (Befragungen) }\end{array}$ & $\begin{array}{l}\text { Abfrage der WTP oder WTA für ein bestimmtes } \\
\text { Gesundheitsrisiko über Items zum Beispiel im Rahmen } \\
\text { einer kontingenten Bewertungsstudie }\end{array}$ \\
\hline
\end{tabular}

Tabelle 3: Ansätze zur Ermittlung einer Zahlungsbereitschaft (U.S. EPA et al., 2003: 4-1ff)

\subsubsection{Methoden zur indirekten Bestimmung einer WTP}

Im Folgenden werden die indirekten Verfahren zur Bestimmung einer WTP erläutert. Diese umfassen im „Children's Health Valuation Handbook“ die „Hedonic Method“ und den „Averting Behavior Approach“. In diesem Zusammenhang wird weiter aufgezeigt, ob sich die einzelnen Methoden zur Ermittlung einer kinderbezogen WTP eignen.

\subsubsection{Die „Hedonic Method“}

Bei der „Hedonic Method“ wird die WTP mittels einer Analyse von Marktpreisdivergenzen indirekt erfasst. Dem Verfahren liegt die Annahme zugrunde, dass ein Gut mehrere objektiv messbare Eigenschaften besitzt, die einen bestimmten Nutzen stiften. Durch Zerlegung des Marktpreises eines Gutes wird den jeweiligen nutzenstiftenden Eigenschaften ein Preis zugeordnet. So kommt es aufgrund von Qualitätsunterschieden in den Umweltattributen bei ansonsten gleichwertigen Gütern zu einer Preisdifferenz, die als „hedonischer Preis“ bezeichnet wird.

Verdeutlicht werden kann diese Vorgehensweise am Beispiel von Immobilienpreisen: Um die WTP für das Gut „Luftqualität“ zu erfassen, wird der Preis eines Wohnhauses in zwei lokal getrennten Regionen miteinander verglichen. Der auftretende Unterschied der Luftqualität kann dann, unter der Annahme ansonsten gleichwertiger nutzenstiftender Merkmale (zum Beispiel die Größe des Hauses, Aufteilung des Hauses), als Preisdifferenz der Wohnhäuser interpretiert werden. 
Eine weitere Möglichkeit besteht darin, über Lohndifferenzen die Wertschätzung des Gutes „Gesundheit“ zu ermitteln. Tätigkeiten mit ähnlichem Anforderungsprofil können sich hinsichtlich ihres Risikos für bestimmte Verletzungen unterscheiden. Die Lohndifferenzen können bei sonst gleichen Tätigkeitsmerkmalen als WTP für die Vermeidung eines erhöhten Verletzungsrisikos auslegt werden.

Ein bedeutender Nachteil der „Hedonic Method“ besteht darin, dass besonders kleine Kinder noch nicht über eigene Einkünfte aus einer beruflichen Tätigkeit verfügen. Folglich kann die kindliche WTP für die Reduzierung von Gesundheitsrisiken auch nicht über Lohndifferenzen ermittelt werden.

Eine Erfassung der WTP über den hedonischen Preis wäre zwar grundsätzlich möglich. Allerdings führt dieses Vorgehen nur zur Feststellung der haushaltsbezogen anstelle einer personenbezogenen WTP. In der haushaltsbezogenen WTP ist der Nutzeneffekt des Kindes zwar enthalten. Eine isolierte Betrachtung der WTP zum Schutz von Kindergesundheit vor schädlichen Umwelteinflüssen ist aber nicht möglich. Hinzu kommt, dass die „Hedonic Method“" nur die gesundheitsrelevanten Handlungen privater Haushalte berücksichtigt. Die positiven Auswirkungen von kollektiven Maßnahmen zum Schutz von Kindergesundheit vor Umweltrisiken werden hingegen nicht einbezogen. Folglich ist die Messung der WTP mittels der „Hedonic Method“ unvollständig, sofern auch öffentliche Güter zur Vermeidung umweltbedingter Erkrankungen von Kindern beitragen (U.S. EPA et al., 2003: 4-2f).

\subsubsection{Der „Averting Behavior Approach“}

Bei der Bestimmung der WTP für den Schutz von Kindergesundheit über den „Averting Behavior Approach“ wird wie bei der „Hedonic Method“ auf bereits existierendes Datenmaterial zurückgegriffen. Damit beschreibt auch der „Averting Behavior Approach“ eine indirekte Methode zur Ermittlung der WTP für den Schutz von Kindergesundheit.

Ausgangspunkt der Betrachtung ist eine substitutive Beziehung zwischen öffentlichen Gütern wie der Umweltqualität und privaten Aufwendungen. Dementsprechend können Individuen private Mittel aufwenden, um umweltbedingte Erkrankungen zu vermeiden. Folglich kann beispielsweise aus der Nachfrage nach gesundheitsfördernden Gütern auf die WTP für die Reduzierung eines Gesundheitsrisikos geschlossen werden (U.S. EPA et al., 2003: 4-3). Da von Kindern aufgrund ihres kognitiven Entwicklungstandes aber keine rationalen Entscheidungen hinsichtlich ihrer Gesundheit erwartet werden können, muss auf die Vermeidungsstrategien der Schutzbeauftragten zurückgegriffen werden, wenn die kinderspezifische WTP ermittelt werden soll. Daher kann die Erfassung der WTP mittels 
„Averting Behavior Approach“ nur aus der elterlichen Perspektive erfolgen (U.S. EPA et al., 2003: 4-3).

Entscheidungen über Investitionen in die Gesundheit von Kindern, wie der Kauf eines Wasserfilters zur Aufwertung verschmutzten Trinkwassers, sind in der Regel aber keine individuellen sondern haushaltsbezogene Entscheidungen. Zum einen weil auch andere Familienmitglieder von der Maßnahme profitieren und zum anderen weil die Entscheidung in Abstimmung mit anderen Familienmitgliedern getroffen wird. Daher ist der „Averting Behavior Approach“ nur begrenzt geeignet, die ausschließlich kinderbezogene WTP für die Reduzierung von umweltbedingten Gesundheitsgefahren wiederzugeben (U.S. EPA et al., 2003: 4-5). Mit der Einbeziehung des familiären Kontextes stellt sich schließlich die Frage, welches Haushaltsallokationsmodell zugrunde gelegt werden muss. So hängt die Wahl des Allokationsmodells davon ab, ob eine bestimmte Vermeidungshandlung nur Auswirkungen auf das Wohlfahrtsniveau eines Kindes oder auf das des gesamten Haushalts hat.

Hinzu kommt, dass Konstellationen denkbar sind, in denen mit einer Vermeidungshandlung gleich mehrere umweltbedingte Erkrankungen verhindert werden können. Ein Beispiel hierfür wäre die Einnahme von Medikamenten zur Stärkung der Immunabwehr. Aus der Nachfrage nach einem solchen Präparat kann kaum die WTP für eine bestimmte umweltbedingte Erkrankung abgelesen werden.

Ein weiteres Problem besteht darin, dass Kaufentscheidungen eher situative als kontinuierliche Handlungsmuster repräsentieren (U.S. EPA, 2000: 1). Die Tatsache, ob Eltern einen Fahrradhelm für ihre Kinder kaufen oder nicht, sagt dementsprechend nicht unmittelbar etwas über ihre generelle Einstellung bezüglich des Schutzes von Kindergesundheit aus. Wird allein aus dem Kaufverhalten die WTP für die Reduzierung von Gesundheitsrisiken für Kinder abgeleitet, kann dies zu Unterschätzungen der wahren Werte führen. Angemessen ist es hingegen, die mittels „Averting Behavior Approach“ erhobene WTP als untere Grenze eines WTP-Intervalls zu interpretieren (U.S. EPA, 2000: 80).

\subsubsection{Methoden zur direkten Bestimmung einer WTP}

Soll die WTP direkt gemessen werden, kann dies über die „Stated Preferences Method“, beispielsweise im Rahmen einer kontingenten Bewertungsstudie erfolgen. Bei der kontingenten Bewertung wird die WTP einer Person für ein nicht-handelbares Gut über Items direkt erfragt.

Der große Vorteil einer direkten Abfrage der WTP besteht in der Flexibilität der Methode. Durch eine bestimmte Itemformulierung kann die Aufmerksamkeit des Befragten gezielt auf 
den Untersuchungsgegenstand, beispielsweise einer Gesundheitsgefährdung von Kindern durch Pestizidrückstände in Lebensmitteln, gelenkt werden. Darüber hinaus können die Befragten einleitend über das zu bewertende Gesundheitsrisiko aufgeklärt werden, um sicher zu stellen, dass die Untersuchungsteilnehmer informierte Entscheidungen treffen können. Im Rahmen der Befragung von Eltern nach ihrer WTP für die Reduzierung eines bestimmten umweltbedingten Gesundheitsrisikos für ihre Kinder ist eine solche Aufklärung zu empfehlen. Eltern haben in der Regel kaum eigene Erfahrungen mit umweltbedingten Erkrankungen und können dadurch die Gefährdung der Kinder häufig nicht richtig einschätzen (U.S. EPA et al, 2003: 4-6).

Die Bestimmung des Nutzens eines Kindergesundheitsprojektes mittels Befragungen ermöglicht, anders als die indirekten Erfassungsmethoden, die Erhebung einer rein kinderbezogenen WTP (U.S. EPA et al., 2003: 4-1). Die Abfrage der WTP für die Reduzierung umweltbedingter Gesundheitsgefahren von Kindern kann grundsätzlich aus der Erwachsene-als-Kinder oder der elterlichen Perspektive erfolgen. Da die Erwachsene-alsKinder Perspektive jedoch kognitiv sehr anspruchvoll für die Befragten ist, ist die Reliabilität der geäußerten WTP tendenziell gering (U.S. EPA et al., 2003: 4-7). Daher ist die elterliche Perspektive vorzuziehen, wenn die kinderspezifische WTP für die Reduzierung von Gesundheitsgefahren erhoben werden soll.

Generell stellt sich jedoch die Frage, ab welchem Alter Kinder in den Bewertungsprozess miteinbezogen werden müssen. Entsprechend ökonomischer Theorien müsste die Meinung von Minderjährigen dann berücksichtig werden, wenn diese in der Lage sind, rationale Entscheidungen zu treffen. Die schrittweise Entwicklung eines Kindes zu einem Erwachsenen erschwert jedoch die Wahl des richtigen Zeitpunkts. So gibt es unsichere Phasen, in der Jugendliche in einigen Bereichen rationales Verhalten zeigen können in anderen jedoch nicht. Die Definition einer allgemeingültigen Altersgrenze erscheint darüber hinaus schwierig, da der kognitive Reifeprozess eines Heranwachsenden von vielen Faktoren abhängig ist. Grundsätzlich sollte jedoch geprüft werden, inwieweit die untersuchte Population bereits Merkmale rationaler Entscheidungsfindung aufweist. Kann davon ausgegangen werden, dass Minderjährige bereits als souveräne Konsumenten in den Markt eingetreten sind, muss ihre Wertschätzung des Guts Gesundheit zumindest in die Zahlungsbereitschaftsanalyse mit einfließen (U.S. EPA et al., 2003: 4-7). 


\subsubsection{Zwischenfazit}

Während im Rahmen der „Hedonic Method“ und des „Averting Behavior Approach“ eine indirekte Erfassung der WTP über bereits vorhandene Marktdaten erfolgt, wird bei der „Stated Preferences Method“ die WTP direkt vom Individuum erfragt. Außerdem führt die Ermittlung der WTP für die Reduzierung von Gesundheitsgefahren für Kinder über die indirekten Methoden immer zu haushaltsbezogen Werten. Hingegen soll die „Stated Preferences Method“ die Erhebung einer ausschließlich kinderbezogenen WTP ermöglichen.

Bezüglich der Bewertungsperspektive ergeben sich weitere Unterschiede zwischen den drei Erhebungsmethoden. So erfolgt die Ermittlung der WTP mittels „Hedonic Method“ und „Averting Behavior Approach“ immer aus der elterlichen Perspektive. Bei der direkten Anfrage der WTP kann zusätzlich auf die Erwachsene-als-Kinder Perspektive zurückgegriffen werden.

Neben den bereits angeführten Unterschieden weisen die Erhebungsverfahren jeweils spezifische Vor- und Nachteile hinsichtlich ihrer Eignung zur Messung kinderspezifischer Nutzeneffekte auf. Die Nachteile der „Hedonic Method“ und des „Averting Behavior Approach“ bestehen in erster Linie in der Unvollständigkeit der Nutzenmessung. Beide Verfahren können nicht die gesamte Wertschätzung des Guts Kindergesundheit erfassen. So berücksichtigen die indirekten Methoden nur die privaten Ausgaben zum Gesundheitsschutz. Die Bereitschaft, zusätzlich in öffentliche Maßnahmen zu investieren, wird hingegen vernachlässigt.

Die Kritik an dem „Stated Preferences“ Konzept und speziell an der kontingenten Bewertungsmethode bezieht sich vor allem auf die praktischen Probleme bei der Datenerhebung. So stellt sich die Frage, ob Eltern ausreichend über die gesundheitsschädliche Wirkung bestimmter Umwelteinflüsse informiert sind, um eine Reduzierung des Risikos reliabel zu bewerten. Problematisch ist diesbezüglich, dass die Bereitstellung von Informationen zum Bewertungsgegenstand zu Einschränkungen der Objektivität der Nutzenmessung führen. Zudem ist bisher unklar, ab welchem Alter Kinder direkt nach einer Zahlungsbereitschaft befragt werden können.

Schließlich kann die Erhebung von Zahlungsbereitschaften über Befragungen immer zu Messfehlern führen. Insbesondere können die Itemformulierungen Ungenauigkeiten bedingen, wenn den Gütekriterien Objektivität, Reliabilität und Validität nicht entsprochen wird. 


\subsection{Ermittlung des Nutzens mittels des Krankheitskostenansatzes}

Alternativ zur Zahlungsbereitschaftsanalyse kann der Nutzen einer Maßnahme zur Reduzierung umweltbedingter Gesundheitsrisiken für Kinder entsprechend des „Children’s Health Valuation Handbook“ auch über die Ermittlung der eingesparten COI erfolgen. Die COI umfassen in der Regel die direkten und indirekten Kosten, die durch eine Erkrankung entstehen. Ein Beispiel für direkte Kosten wäre Ausgaben für stationäre und medizinische Behandlungen ${ }^{14}$. Für die indirekten Kosten ist eine verminderte Produktivität am Arbeitsplatz relevant.

Bei der Kalkulation von Behandlungskosten ist entsprechend des „Children's Health Valuation Handbooks“ darauf zu achten, dass die verwendeten Werte auf aktuellen Daten beruhen, da die Behandlungskosten aufgrund des schnellen medizinischen Fortschritts einem ständigen Wandel unterliegen. Des Weiteren ist sicherzustellen, dass das zugrunde gelegte Datenmaterial eventuelle Kostenunterschiede bei der Behandlung von Erwachsen und Kinder berücksichtigt, wenn kinderspezifische Nutzeneffekt ermittelt werden sollen (U.S. EPA et al., 2003: 4-7).

Eine Schwierigkeit besteht darin, die Zeitverluste durch die Erkrankung eines Kindes zu erfassen. Ist ein Kind erkrankt, „kostet“ dies nicht nur die Zeit des Kindes selbst, sondern auch die des Erwachsenen die es beaufsichtigen müssen. Die Zeitverluste des Erwachsenen lassen sich über die Produktivitätsverluste am Arbeitsplatz monetär bewerten. Probleme bereitet hingegen die ökonomische Bewertung der durch Krankheit verlorenen Zeit des Kindes. Chronische Erkrankungen können zu einer erheblichen Anzahl von Fehltagen in der Schule oder einer verkürzten Lebenserwartung führen. Diese Kosten des Kindes müssen bei der Ermittlung der COI berücksichtigt werden und zwar durch die diskontierten Einkommensverluste des Kindes im späteren Erwachsenalter. Als Anhaltspunkt für die zu erwartenden Einkommensverluste, kann das Verhältnis von Intelligenzquotient und der damit zu prognostizierenden Einkommenshöhe dienen (U.S. EPA et al., 2003: 4-8).

Der entscheidende Nachteil des COI-Ansatzes besteht in der Vernachlässigung der immateriellen Kosten. Aufgrund dessen eignet sich die Ermittlung der COI nicht zur Messung von Wohlfahrtsänderungen (U.S. EPA et al., 2003: 4-10).

Wichtig ist es auch darauf hinzuweisen, dass es sich bei dem COI-Ansatz um eine ex post und nicht um eine ex ante Messung des Nutzens einer Reduzierung von Gesundheitsrisiken handelt. Ein Vorteil der COI-Ermittlung zur Nutzenerfassung gegenüber der WTP besteht

\footnotetext{
${ }^{14}$ Die Behandlungskosten lassen sich nochmals in Sach- und Personalkosten aufschlüsseln.
} 
allerdings in der vergleichsweise einfachen Durchführbarkeit und weitgehenden Akzeptanz unter Experten (U.S. EPA et al., 2003: 4-10).

\subsubsection{Zwischenfazit}

Soll der Nutzen einer politischen Maßnahme zur Reduzierung umweltbedingter Gesundheitsgefahren von Kindern gemessen werden, können grundsätzlich die Zahlungsbereitschaftsanalyse wie auch der COI-Ansatz eingesetzt werden. Möchte man jedoch Aussagen über die ökonomische Effizienz eines Programms treffen und damit Rückschlüsse auf eine potentielle Pareto-Verbesserung ziehen, kommt hierfür nur die Zahlungsbereitschaftsmethode in Betracht. Nur durch die Einbeziehung der WTP sind alle Nutzeneffekte, auch die immateriellen, zu erfassen.

\subsection{Bewertungsunterschiede zwischen Kindern und Erwachsenen}

Entsprechend des „Children’s Health Valuation Handbooks“ soll die monetäre Bewertung von Kindergesundheit über die Ermittlung der WTP oder ersatzweise über die COI erfolgen (U.S. EPA et al., 2003: 2-5). Allerdings bestehen zwischen Kindern und Erwachsenen erhebliche Bewertungsunterschiede, die auf Altersdifferenzen zwischen Kindern und Erwachsenen beruhen. Im „Children’s Health Valuation Handbook“ wird hierzu einerseits diskutiert, aus welcher Perspektive die Erhebung kinderspezifischer WTP erfolgen soll, wenn Kinder nicht direkt befragt werden können. Anderseits wird dargelegt, wie WTP und COI von Erwachsenen und Kindern differieren und worin diese Unterschiede begründet sind.

\subsubsection{Die Wahl der Perspektive}

Sollen gesundheitliche Beeinträchtigungen von Kindern monetär bewertet werden, stehen Ökonomen vor dem Problem, die Kinder als unmittelbar Betroffene nicht direkt befragen zu können. Aufgrund ihres kognitiven Entwicklungsstandes entsprechen Kindern nicht der grundlegenden Annahme eines rationalen Entscheidungsträgers ${ }^{15}$. Daher stellt sich die Frage, wer anstelle des Kindes nach der WTP befragt werden soll, beziehungsweise aus welcher Perspektive Kindergesundheit angemessen $\mathrm{zu}$ bewerten ist. Diesbezüglich werden im „Children's Health Valuation Handbook“ drei Alternativen gegenübergestellt und anschließend diskutiert.

Zunächst ist die soziale Perspektive zu nennen, bei der alle erwachsenen Mitglieder einer Gesellschaft, also sowohl Eltern als auch kinderlose Erwachsene, nach ihrer WTP für die

\footnotetext{
${ }^{15}$ Siehe hierzu auch Kapitel 4.
} 
Reduzierung umweltbedingter Gesundheitsrisiken für Kinder befragt werden. Als weitere Option kommt die Erwachsene-als-Kinder Perspektive in Betracht, bei der Erwachsene gebeten werden, sich in die Rolle eines Kindes zu versetzen und aus dieser Perspektive Gesundheitseffekte zu bewerten. Schließlich besteht eine weitere Möglichkeit darin, die elterliche Perspektive zu wählen. Dabei werden Eltern gebeten eine WTP für die Reduzierung einer Gesundheitsgefahr ihres eigenen Kindes anzugeben (U.S. EPA et al., 2003: 2-7; 2-10).

Bei kritischer Betrachtung zeigt sich, dass mit allen drei genannten Perspektiven bestimmte Vor- und Nachteile verbunden sind. Während es bei der sozialen Perspektive durch altruistische Motive zu Verzerrungen, insbesondere zu Überschätzungen der WTP kommen kann, die kaum zu kontrollieren sind, führt die Erwachsene-als-Kinder Perspektive häufig zu kognitiver Überforderung der Befragten und Schwierigkeiten bei der Itementwicklung (U.S. EPA et al., 2003: 2-8). Zudem wird mit der Erwachsene-als-Kinder Perspektive eine ex post Bewertung von Gesundheitsrisiken anstelle der im politischen Kontext angestrebten ex ante Bewertung vorgenommen.

Auch die elterliche Perspektive ist mit spezifischen Nachteilen belastet, welche aus der Einbeziehung Dritter in den Bewertungsprozess resultieren. So kann es einerseits zu Überschätzungen der WTP kommen, wenn Eltern die Gesundheit aufgrund von elterlichem Altruismus höher bewerten als die betroffenen Kinder dieses selbst tun würden. Andererseits können sich Unterschätzungen der WTP ergeben, wenn Eltern in Extremfällen nur ihren individuellen Nutzen, den ein gesundes Kind für sie persönlich mit sich bringt, bewerten (U.S. EPA et al., 2003: 2-9). Des Weiteren können Informationsdefizite der Eltern in Bezug auf die gesundheitlichen Auswirkungen bestimmter Umwelteinflüsse bei Kindern Verzerrungen der WTP bedingen (U.S. EPA et al., 2003: 2-9f).

Schließlich bewerten Eltern die Reduzierung eines Gesundheitsrisikos immer unter Berücksichtigung ihrer persönlichen finanziellen Lage. Allerdings muss davon ausgegangen werden, dass die derzeitigen Kinder im Vergleich zu ihren Eltern ein höherer finanzieller Wohlstand im Erwachsenalter erwartet. Hierfür können Eltern keine „Vorauszahlung“ leisten. Aufgrund dieser unterschiedlichen finanziellen Voraussetzungen bewerten Eltern die Gesundheit ihres Kindes niedriger, als es das Kind selbst als hypothetischer Erwachsener tun würde (U.S. EPA et al., 2003: 2-10).

Ein entscheidender Vorteil der elterlichen Perspektive gegenüber der gesamtgesellschaftlichen Sichtweise besteht in der engen emotionalen Verbundenheit der Eltern zu ihrem Kind. Aufgrund dessen kann davon ausgegangen werden, dass Eltern den bestmöglichen Gesundheitsschutz für ihre Kinder anstreben, ähnlich wie diese es selbst tun 
würden. Schließlich berücksichtigt die elterliche Perspektive, dass es sich bei vielen Entscheidungen bezüglich des Gesundheitsschutzes vor schädlichen Umwelteinflüssen nicht um individuelle Entscheidungen handelt. Vielmehr betreffen Investitionen in die Reduzierung von Gesundheitsrisiken meist eine Familie als Ganzes, wenn auch in unterschiedlichem Ausmaß (U.S. EPA et al., 2003: 2-9f).

\subsubsection{Unterschiede bei WTP und COI zwischen Kindern und Erwachsenen}

Ähnlich wie die Abschätzung des kinderspezifischen Risikos durch negative Umwelteinflüsse aufgrund des Mangels an epidemiologischen Daten erschwert wird, führt auch das Fehlen kinderspezifischer WTP- und COI-Werte $\mathrm{zu}$ Problemen bei der Ermittlung des wohlfahrtökonomischen Nutzens umwelt- und gesundheitspolitischer Interventionen. Analog zur Risikoabschätzung stellt sich daher auch im Bereich der monetären Bewertung die Frage, ob erwachsenenbezogene WTP und COI auf Kinder übertragen werden dürfen (Benefit Transfer).

Zahlreiche Untersuchungen zeigen jedoch, dass sowohl die WTP als auch die COI zum Teil erhebliche altersabhängige Unterschiede aufweisen ${ }^{16}$. Verantwortlich dafür sind die folgenden drei Einflussgrößen:

- Risikobereitschaft

- $\quad$ Alters- und Zeiteffekte

- Behandlungskosten

Auf diese drei Faktoren und ihren Einfluss auf die WTP und COI wird im Folgenden näher eingegangen.

\subsubsection{Risikobereitschaft}

Es gibt zahlreiche Hinweise darauf, dass sich Eltern und auch eine Gesellschaft als Ganzes vergleichsweise risikoaverser verhalten, wenn es um die Gesundheit von Kindern geht. Beispiele hierfür sind bestimmte Gesetze, die ausschließlich dem Schutz von Kindergesundheit dienen sollen und keine Gültigkeit für Erwachsene besitzen. Auch die Bemühungen von Eltern, Kinder in besonderem Maße vor Gesundheitsrisiken zu schützen, sind hierfür exemplarisch. Diese geringere Risikobereitschaft gegenüber der Gesundheit von Kindern lässt darauf schließen, dass Kindergesundheit höher bewertet wird als die von Erwachsenen und zwar unabhängig von der gewählten Perspektive (U.S. EPA et al., 2003: 212f).

\footnotetext{
${ }^{16}$ Siehe hierzu auch Kapitel 6.3.6.
} 
Auch der Grad der Freiwilligkeit, mit der ein Risiko eingegangen wird, hat Einfluss auf die individuelle Risikobereitschaft. Auf wissenschaftlicher Seite wird grundsätzlich davon ausgegangen, dass Personen die Reduzierung eines unfreiwilligen Risikos der Minimierung eines freiwilligen Risikos vorziehen (FISCHHOFF et al., 1978; SLOVIC, 1987). Gerade kleine Kinder, welche von den Entscheidungen ihrer Eltern abhängig sind, sind fast ausschließlich mit unfreiwilligen Risiken konfrontiert. Folglich kann angenommen werden, dass die Bewertung von Gesundheitsrisiken über Stellvertreter dazu führt, dass für Kinder eine höhere WTP angegeben wird als für Erwachsene (U.S. EPA et al., 2003: 2-12f).

Des Weiteren hat auch die Unsicherheit, die mit einer bestimmten umweltbezogen Gesundheitsgefahr für Kinder einhergehen kann, Auswirkungen auf die Höhe der WTP für die Reduzierung des Risikos. Im Allgemeinen versuchen Personen Unsicherheiten zu vermeiden und bevorzugen daher die Abwendung einer Risikosituation mit ungewissem Ausgang gegenüber einer Situation mit relativ sicherem Ausgang (VISCUSI et al., 1991). Viele Umwelteinflüsse sind hinsichtlich ihrer Auswirkungen auf die Gesundheit von Kindern aber stärker mit Unsicherheiten behaftet als bei Erwachsenen. Daher ist anzunehmen, dass die WTP für die Reduzierung eines umweltbezogenen Gesundheitsrisikos für Kinder generell höher ausfällt (U.S. EPA et al., 2003: 2-13).

\subsubsection{Alters- und Zeiteffekte}

Weitere Bewertungsunterschiede können aus den Altersunterschieden zwischen Kindern und Erwachsenen resultieren. Im Einzelnen sind hierfür die nachstehend genannten Einzelaspekte verantwortlich:

a die größere Anzahl verbleibender Lebensjahre

b das zukünftig höhere Wohlstandsniveau der jetzigen Kinder im Erwachsenenalter

c die Diskontierungsrate, insbesondere bei latent wirksamen Gesundheitseffekten

Hinsichtlich der Alterunterschiede zwischen Kindern und Erwachsenen ist anzumerken, dass die größere Anzahl verbleibender Lebensjahre bei Kindern aus verschiedenen Gründen zu einer erhöhten WTP führen. Einerseits verlieren Kinder durch einen vorzeitigen Tod infolge negativer Umwelteinflüsse vergleichsweise mehr Lebenszeit. Andererseits verbleibt bei Kindern aufgrund ihres geringeren Lebensalters mehr Zeit, um latent wirkende Krankheiten zu entwickeln oder unter chronischen Erkrankungen zu leiden (U.S. EPA et al., 2003: 2-14). Die längere Krankheitsdauer und die erhöhte Realisierungswahrscheinlichkeit latenter Gesundheitseffekte bewirken darüber hinaus zusätzlich einen Anstieg der COI. 
Der zu erwartende höhere Wohlstand der derzeitigen Kinder im späteren Erwachsenenalter beeinflusst ebenfalls die WTP für die Reduzierung eines Gesundheitsrisikos. Der wachsende gesellschaftliche Wohlstand geht mit einem Anstieg des Lohnniveaus einher. Dieser Anstieg bewirkt wiederum eine höhere WTP der zukünftigen Erwachsen im Vergleich zur aktuellen Erwachsenengeneration (U.S. EPA et al., 2003: 2-14f). Wird die WTP für die Reduzierung von Gesundheitsgefahren für Kinder über Stellvertreter erhoben, muss der Einfluss zukünftig steigender Löhne berücksichtigt werden, um Unterschätzungen der WTP zu vermeiden. Sollen erwachsenenspezifische Daten auf Kinder übertragen werden, sind die Werte entsprechend an den neuen Bewertungskontext anzupassen, um aussagekräftige WTP zu erhalten (U.S. EPA et al., 2003: 2-15).

Die monetäre Bewertung latenter Gesundheitsrisiken erfordert generell eine Anpassung der WTP durch Diskontierung. Werden umweltbedingte Gesundheitsrisiken erst mit einer zeitlichen Verzögerung wirksam, muss diese Zeitspanne durch Abzinsung der WTP in die Kosten-Nutzen-Analyse einbezogen werden ${ }^{17}$. Ist die Phase zwischen Exposition und Reaktion des menschlichen Körpers so kurz, dass Kinder und Erwachsene in gleicher Weise von einer Gesundheitsschädigung bedroht sind, können sich die jeweiligen Diskontierungsraten trotzdem unterscheiden. So ist es denkbar, dass Eltern für ihre Kinder andere Diskontierungsraten zugrunde legen als für sich selbst (AGEE und Crocker, 1996; TOLLEY und FABIAN, 1999) ${ }^{18}$. Diese Fragestellung ist bisher jedoch wenig untersucht und es wird weitere Forschung benötigt, um fundierte Aussagen zur Altersabhängigkeit der Diskontierungsrate treffen zu können.

\subsubsection{Behandlungskosten}

Bei den Krankheitskosten, die durch negative Umwelteinflüsse versucht werden, ist mit weiteren Unterschieden zwischen Kindern und Erwachsenen zu rechnen. So können die Behandlungskosten für die resultierenden gesundheitlichen Beeinträchtigungen zwischen Kindern und Erwachsen differieren, sofern sich Art und/oder Dauer der Behandlung unterscheiden. Die Frage, in welche Richtung Abweichungen der COI zu erwarten sind, kann nicht allgemeingültig beantwortet werden. Ob die Kosten für Kinder höher oder niedriger zu kalkulieren sind, ist letztlich vom betrachteten Krankheitsbild abhängig.

\footnotetext{
${ }^{17}$ Zur Berechnung der Diskontierungsrate siehe auch SCHÖFFSKI et al. (2008: 178ff) und BRANDES et al. (2001).

${ }^{18}$ Die intergenerationelle Diskontierung muss hier nicht weiter betrachtet werden, da sich das „Children's Health Valuation Handbook“ auf die ökonomische Bewertung von Gesundheitseffekte der aktuell lebenden Kinder bezieht und nicht auf die zukünftigen Generationen.
} 
Schließlich hat auch die Zeit, die die Erkrankung eines Kindes beansprucht, sowohl Einfluss auf die Höhe der WTP als auch die der COI. In zeitlicher Hinsicht betrifft die Erkrankung eines Kindes nicht nur dieses selbst, sondern auch die Erwachsenen, die das erkrankte Kind betreuen. Dementsprechend müssen sowohl die zeitlichen Verluste der Betreuer und die des Kindes monetär bewertet werden. Aufgrund dieses doppelten Zeiteffekts kann für Kinder von einer höheren WTP zur Vermeidung umweltbedingter Erkrankungen ausgegangen werden als bei Erwachsenen.

\subsubsection{Zwischenfazit}

Im „Children's Health Valuation Handbook“ werden zahlreiche Bewertungsunterschiede zwischen Kindern und Erwachsenen offen gelegt. Grundsätzlich gilt, dass das Vorliegen der angeführten Bewertungsunterschiede eine gesonderte Kosten-Nutzen-Analyse für Kinder notwendig erscheinen lässt.

Von den drei genannten möglichen Bewertungsperspektiven zur Erfassung einer kinderspezifischen WTP sind nur die elterliche Perspektive und eingeschränkt die Erwachsene-als-Kinder Perspektive empfehlenswert. Von der sozialen Perspektive ist hingegen abzuraten, weil die vorgenommenen Bewertungen durch unterschiedliche Formen von Altruismus beeinflusst sein können. Da weder die Art noch das Ausmaß des Einflusses altruistischer Motive quantitativ bestimmbar ist, kann die ermittelte WTP auch nicht um den verzerrenden Effekt bereinigt werden. Als Folge können durch Doppelzählungen erhebliche Überschätzungen der WTP für den Schutz von Kindergesundheit resultieren.

\subsection{Alternative Ansätze zur Bewertung umweltpolitischer Kindergesundheits- projekte}

Sind zuverlässige Schätzungen der WTP oder des bestehenden Gesundheitsrisikos für Kinder nicht möglich, kann auf alternative Formen der Wirtschaftlichkeitsuntersuchungen zur Prüfung der Vorteilhaftigkeit einer umweltpolitischen Maßnahme zurückgegriffen werden. Diesbezüglich werden im „Children’s Health Valuation Handbook“ vier unterschiedliche Verfahren vorgestellt gestellt. Dies sind im Einzelnen die Kosten-Wirksamkeits-Analyse (Cost-Effectiveness-Analysis), die Break Even Analyse, die Bounding Analysis sowie die Health-Health- und die Risk-Risk-Analysis (U.S. EPA et al., 2003: 5-1ff). Diese Bewertungsmethoden werden in den nächsten Abschnitten beschrieben. 


\subsubsection{Kosten-Wirksamkeits-Analysen}

Bei der Kosten-Wirksamkeits-Analyse handelt es sich um ein Instrument zur Prüfung der Wirtschaftlichkeit einer Projektvariante. Die Ermittlung der Kosten erfolgt analog zur Kostenkalkulation in der Kosten-Nutzen-Analyse. Die Nutzenkomponente wird hingegen nicht direkt monetär gemessen, sondern durch die Anzahl der vermiedenen Todes- oder Krankheitsfälle ausgedrückt.

Eine häufig in der Gesundheitsökonomie angewandte Unterform der Kosten-WirksamkeitsAnalyse ist die Kosten-Nutzwert-Analyse (Cost-Utility-Analysis). Bei der Kosten-NutzwertAnalyse werden die Nutzeneffekte einer umwelt- oder gesundheitspolitischen Maßnahme durch die qualitätskorrigierten Lebensjahre (in englisch: Quality Adjusted Life Years, QALYs) oder die behinderungsbereinigten Lebensjahre (in englisch: Disability Adjusted Life Years, DALYs) wiedergegeben ${ }^{19}$. Bei den DALYs und QALYs handelt es sich um Kennzahlen, die neben der Lebensdauer auch die Lebensqualität berücksichtigen. Ein QALY von eins bedeutet ein Lebensjahr in völliger Gesundheit. Ein QALY von Null repräsentiert hingegen den Tod. Eine Kosten-Nutzwert-Analyse erlaubt damit Aussagen darüber, welche umweltpolitische Maßnahme den größten Nutzen verspricht (U.S. EPA et al., 2003: 5-2). Damit eignet sich die Kosten-Nutzwert-Analyse besonders zur Entscheidung über zwei sich ausschließende Alternativen.

Der große Nachteil der Kosten-Nutzwert-Analyse besteht darin, dass die wirksamste Alternative nicht auch effizient im ökonomischen Sinn sein muss. So sind Konstellationen denkbar, in denen das Verhältnis von Kosten pro QALY bei Alternative A zwar günstiger ausfällt als bei Alternative B. Allerdings sagt die Vorteilhaftigkeit von Alternative A gegenüber B noch nichts über die ökonomische Effizienz von A aus. Um auf den Beitrag der Alternativen zur Wohlfahrtsmaximierung folgern zu können, müssten alle Kosten und der gesamte Nutzen quantifiziert und gegenübergestellt werden. Da die Nutzenmessung im Rahmen der Kosten-Nutzwert-Analyse aber nicht monetär erfolgt, ist der unmittelbare Abgleich von Kosten und Nutzen nicht möglich. Das bedeutet, dass auf der Basis von KostenWirksamkeits-Analysen keine Aussagen über Änderungen der sozialen Wohlfahrt infolge einer Implementierung umweltpolitischer Maßnahmen zum Schutz von Kindergesundheit formuliert werden können.

Eine weitere Schwierigkeit der Kosten-Nutzwert-Analyse ergibt sich aus der präferenzbasierten Erhebung der QALYs und DALYs. Wenn es um die Bewertung von

\footnotetext{
${ }^{19}$ Zum Konzept des QALY und DALY sowie zu deren Ermittlung siehe SCHÖFFSKI (2008: 95ff).
} 
Kindergesundheit geht, stellt sich wie bei der Kosten-Nutzen-Analyse die Frage, aus welcher Perspektive die Nutzenbestimmung erfolgen soll (U.S. EPA et al., 2003: 5-4).

\subsubsection{Break Even Analyse}

Eine Break Even Analyse kann zur Untersuchung der Wirtschaftlichkeit einer gesundheitsund umweltpolitischen Maßnahme eingesetzt werden, wenn entweder die Anzahl reduzierbarer Krankheitsfälle oder die Wertschätzung des Guts Gesundheit für eine bestimmte Population nicht ermittelt werden kann. Für den Fall, dass Risikodaten nicht vorhanden sind, gibt die Break Even Analyse unter Einbeziehung der WTP für eine Risikoreduzierung Auskunft darüber, wie viel Todes- oder Krankheitsfälle durch eine Schutzmaßnahme bei gegebenen Kosten mindestens reduziert werden müssen, damit die Intervention gewinnbringend ist. Fehlt hingegen die WTP für die Vermeidung gesundheitlicher Beeinträchtigungen, kann bei Berücksichtigung der Anzahl vermeidbarer Krankheitsfälle schlussgefolgert werden, wie hoch die Wertschätzung des Guts Gesundheit mindestens ausfallen müsste, damit eine Intervention lohnend ist (U.S. EPA et al., 2003: 5-3). Damit zeigt eine Break Even Analyse den Punkt auf, der die Gewinn- von der Verlustzone trennt (Break Even Point).

Die Break Even Analyse ist dann unkompliziert durchführbar, wenn eine umwelt- oder gesundheitspolitische Maßnahme nur auf die Reduzierung eines Gesundheitseffektes abzielt. Sollen mehrere verschiedene Erkrankungen mittels einer Intervention vermieden werden, ergeben sich Probleme bei der Umsetzung. In solchen Fällen erfordert die Betrachtung verschiedener Gesundheitseffekte die Festsetzung einer Break Even Grenze, die eine Variation der reduzierten Fälle pro Erkrankung ermöglicht (U.S. EPA et al., 2003: 5-3).

\subsubsection{Bounding Analysis}

Die Bounding Analyse stellt ein geeignetes Instrument zur Prüfung der ökonomischen Vorteilhaftigkeit einer umweltpolitischen Schutzmaßnahme für Kinder dar, wenn die WTP für die Vermeidung einer bestimmten Erkrankung nicht feststellbar ist, jedoch Daten für eindeutig schwerwiegendere oder weniger schwere Krankheitsbilder vorliegen. Vor dem Hintergrund, dass die Reduzierung stärkerer gesundheitlicher Beeinträchtigungen im Allgemeinen höher bewertet wird als die Vermeidung weniger schwerer Fälle, sollte der fehlende Wert zwischen diesen beiden WTP-Werten liegen. Somit stellt die WTP für die Vermeidung der schwerwiegenderen Erkrankung die oberste Grenze und die WTP der weniger schweren Erkrankung die untere Grenze des Intervalls dar, in dem die unbekannte 
WTP für zu finden ist. Durch Gegenüberstellung des Intervalls mit den zu erwartenden Kosten, lassen sich Hinweise auf die Effizienz des Programms ableiten.

In Anbetracht der Tatsache, dass nur wenig Daten für die Reduzierung kinderspezifischer Gesundheitsgefahren vorhanden sind, kann es allerdings schwierig sein, einen Bereich zu identifizieren, in dem sich der fehlende WTP-Wert vermutlich befindet (U.S. EPA et al., 2003: 5-4).

\subsubsection{Risk-Risk- and Health-Health-Analysis}

Im Rahmen von Risk-Risk- und Health-Health-Analysis findet keine Monetarisierung des Nutzens eines politischen Programms statt. Auch die Kosten, welche mit der Umsetzung einer umweltpolitischen Maßnahme zur Reduzierung von Gesundheitsrisiken für Kinder verbunden sind, werden nicht in Geldeinheiten angegeben. Stattdessen wird bei der Risk-Risk-Analysis das Ausmaß anhand einer umweltpolitischen Maßnahme erreichbaren Risikoreduktion und der unbeabsichtigten Risikoerhöhung infolge der Intervention gegenübergestellt (U.S. EPA et al., 2003: 5-4).

Bei der Health-Health-Analysis wird anstelle der $\mathrm{zu}$ erwartenden Variationen der Erkrankungsrisiken die Anzahl der verhinderten beziehungsweise unbeabsichtigt erhöhten Todesfälle miteinander verglichen (U.S. EPA et al., 2003: 5-4).

Hinter diesem Ansatz steht die Überlegung, dass jede Maßnahme zur Reduzierung umweltbedingter Todesfälle mit finanziellen Aufwendungen verbunden ist. Die Finanzierung dieser kollektiven Programme erfolgt über die Privathaushalte. Infolgedessen steht den Individuen eine geringere Geldmenge für private Schutzmaßnahmen zur Verfügung. Der Rückgang privater Bemühung zum Schutz der Gesundheit kann jedoch zu einem unerwünschten Anstieg von Todesfällen führen. Dieser unbeabsichtigte Nebeneffekt muss bei der Prüfung der Wirksamkeit einer politischen Intervention berücksichtigt werden.

Ein schwerwiegender Nachteil der Health-Health-Analysis besteht darin, dass hier nur die Anzahl der Todesfälle in die Betrachtung einbezogen wird. Andere, nicht tödliche Gesundheitseffekte, werden dagegen komplett vernachlässigt. Aufgrund dessen ist die HealthHealth-Analysis nur für die Untersuchung von Sachverhalten geeignet, bei der ausschließlich das frühzeitige Versterben infolge negativer Umwelteinflüsse betrachtet werden soll. Sind hingegen auch andere nicht tödliche Gesundheitseffekte für den Bewertungskontext relevant, führt eine Health-Health-Analysis zu fehlerhaften Ergebnissen (U.S. EPA et al., 2003: 5-4f). 


\subsubsection{Zwischenfazit}

Im vorhergehenden Kapitel wurden unterschiedliche Bewertungsverfahren vorgestellt, die Auskunft über die Vorteilhaftigkeit eines Kindergesundheitsprojekts geben können. Diese Bewertungsansätze können angewandt werden, wenn die Durchführung einer Kosten-NutzenAnalyse aufgrund fehlender Daten nicht möglich ist. Die benannten Verfahren haben den Vorteil, dass sie anders als die Kosten-Nutzen-Analyse entweder nur Daten zur Erkrankungswahrscheinlichkeit von Kindern oder zur WTP benötigen. Ihr Nachteil gegenüber der Kosten-Nutzen-Analyse besteht darin, dass keine direkte Gegenüberstellung von Kosten und (monetarisiertem) Nutzen erfolgt. Daher kann mit keinem der vorgestellten alternativen Bewertungsansätze die ökonomische Effizienz von umweltpolitischen Kindergesundheitsprojekten bestimmt werden.

Zwar liegt der Kosten-Wirksamkeits-Analyse, der Break Even Analyse und der Bounding Analysis teilweise eine Monetarisierung von Aufwand und/oder Ergebnis zugrunde. Die zu erwartenden Kosten und der Nutzen eines Kinderschutzprojektes, werden aber nicht in der gleichen Einheit miteinander verglichen. Im Rahmen der Risk-Risk- und Health-HealthAnalysis erfolgt gar keine Überführung der Komponenten in Geldeinheiten. Folglich können auch keine Aussagen über die Wirtschaftlichkeit einer Projektvariante getroffen werden. Zudem dürfen die Kosten-Wirksamkeits-Analysen, die Break Even Analyse und die HealthHealth-Analysis nur eingesetzt werden, wenn lediglich ein ausgewähltes Krankheitsbild betrachtet werden soll. Da die meisten umweltpolitischen Programme zum Schutz von Kindergesundheit jedoch multiple Nutzeneffekte aufweisen, ist der Anwendungsbereich dieser Analyseverfahren stark eingeschränkt (U.S. EPA et al., 2003: 5-4f).

Sollen Aussagen über eine potentielle Pareto-Verbesserung infolge einer umweltpolitischen Maßnahme zum Schutz von Kindergesundheit getroffen werden, eignet sich also keines der dargestellten Verfahren dazu, die Kosten-Nutzen-Analyse zu ersetzen. Ist die Durchführung einer Kosten-Nutzen-Analyse aufgrund von Datenmangel jedoch nicht möglich, sollte aber trotzdem auf die alternativen Bewertungsverfahren zurückgegriffen werden, um Hinweise auf die Wirksamkeit umweltpolitischer Programme zu erhalten.

\subsection{Benefit Transfer}

Das Fehlen kinderspezifischer Daten erfordert oft die Übertragung (Benefit Transfer) einer WTP aus anderen Bewertungskontexten. Prinzipiell muss bei der Übernahme von Daten danach unterschieden werden, ob Werte von Erwachsenen auf Kinder oder Werte von Kindern auf Kinder übertragen werden. Die Übertragung von erwachsenenbezogenen WTP- 
Werte auf einen kinderspezifischen Bewertungskontext kann aus verschiedenen Gründen notwendig sein. Zum einen, weil die direkte Ermittlung spezieller Daten für Kinder zu teuer und zu zeitintensiv wäre. Zum anderen, da kinderspezifische Werte, welche übernommen werden könnten, nicht vorhanden sind.

Im Vordergrund der Ausführungen des „Children’s Health Valuation Handbook“ steht der Transfer von erwachsenenbezogenen WTP auf Kinder $^{20}$. Hierzu wird ein vierstufiges Verfahren vorgeschlagen, welches in Tabelle 4 zusammengefasst ist.

\begin{tabular}{|c|c|}
\hline Phasen des Wertetransfers & Arbeitsschritte \\
\hline $\begin{array}{l}\text { 1. Beschreibung des } \\
\text { umweltpolitischen Problems }\end{array}$ & $\begin{array}{l}\text { - } \text { Identifikation der Gesundheitseffekte } \\
\text { - Charakterisierung der Gesundheitseffekte } \\
\text { - Bestimmung der Auswirkungen auf das Wohlergehen } \\
\text { - Beschreibung der Populationscharakteristika }\end{array}$ \\
\hline $\begin{array}{l}\text { 2. Beurteilung der Brauchbarkeit } \\
\text { vorhandener Studien }\end{array}$ & $\begin{array}{l}\text { - } \quad \text { Prüfung der Studienqualität } \\
\text { - } \quad \text { Prüfung der Studienähnlichkeit }\end{array}$ \\
\hline 3. Übertragung der Daten & $\begin{array}{ll}\text { - } & \text { Transfer von Punktschätzungen oder } \\
& \text { Nutzenfunktionen } \\
\text { - } & \text { Anpassung der Werte mittels geeigneter Verfahren }\end{array}$ \\
\hline $\begin{array}{l}\text { 4. Qualitative Beschreibung von } \\
\text { Unsicherheiten }\end{array}$ & $\begin{array}{l}\text { - Qualitative Darstellung der möglicher Fehlerquellen } \\
\text { im Rahmen der Datenübertragung, sofern eine } \\
\text { Quantifizierung nicht möglich ist }\end{array}$ \\
\hline
\end{tabular}

Tabelle 4: Phasen des Benefit Transfer(U.S. EPA et al., 2003: 3-3)

\subsubsection{Phase 1: Beschreibung des umweltpolitischen Problems}

Die erste Phase der Datenübertragung dient der Aufschlüsslung des zu untersuchenden umwelt- oder gesundheitspolitischen Problems und ist in vier Teilschritte gegliedert.

\subsubsection{Teilschritt 1: Identifikation der Gesundheitseffekte}

Der erste Teilschritt bezieht sich auf die Erfassung gesundheitlicher Beeinträchtigungen. Konkret geht es um die Klärung der Frage, wie Gesundheits- und Wohlfahrteffekte definiert sind und gemessen werden. $\mathrm{Zu}$ beachten ist hier, dass Expositionen gegenüber der gleichen Umweltkontamination nicht nur zu unterschiedlichen Erkrankungen führen können, sondern

\footnotetext{
${ }^{20}$ Es sind jedoch auch einige Hinweise für die Übertragung von Werte aus kinderspezifischen Studien auf Kinder in anderen Bewertungskontexten enthalten.
} 
dass der gleiche physische Effekt abweichende Auswirkungen auf das individuelle Wohlergehen von Kindern und Erwachsenen haben $k^{2}{ }^{21}$. Weiterhin ist zu prüfen, ob die angewandte Methode zur Messung der Wohlfahrteffekte in der erwachsenenbezogenen Originalstudie auch zur Erfassung von Wohlfahrtseffekten bei Kindern geeignet ist. Die Messung von Wohlfahrtseffekten über die Vermeidung von Einkommensverlusten ist für die Ermittlung des kinderspezifischen Nutzens nicht geeignet (U.S. EPA et al., 2003: 3-4).

\subsubsection{Teilschritt 2: Charakterisierung der Gesundheitseffekte}

Im zweiten Teilschritt steht die ausführliche Charakterisierung der möglichen gesundheitlichen Beeinträchtigungen durch eine ausgewählte Umweltkontamination im Vordergrund. Hierbei geht es um die Klärung fünf grundlegender Fragen, die die Risikounterschiede zwischen Kindern und Erwachsenen betreffen. Zunächst muss die Eintrittswahrscheinlichkeit eines Gesundheitseffekts festgestellt werden. Sind die Realisierungschancen einer Erkrankung in der Originalstudie und der geplanten Analyse nicht identisch, müssen die Werte modifiziert werden. Da die WTP bei einer geringen Reduzierung eines Gesundheitsrisikos eine lineare Beziehung zum Ausmaß des Risikos aufweist, können einzelne Werte pro Reduzierungseinheit berechnet werden (beispielsweise 3 Euro pro einprozentiger Reduzierung des Gesundheitsrisikos). Der lineare Zusammenhang zwischen WTP und dem Ausmaß der Risikoreduzierung gilt jedoch nur bei kleinen Wahrscheinlichkeitsänderungen (U.S. EPA et al., 2003: 3-5).

Des Weiteren ist zu klären, ob die durch eine politische Intervention angestrebte Reduzierung des Gesundheitsrisikos hinsichtlich Häufigkeit, Schwere und Dauer der Erkrankungen in gleicher Weise zum Schutz der Gesundheit von Kindern und Erwachsenen beiträgt.

Als nächstes ist zu prüfen, inwieweit die vorliegenden Gesundheitseffekte durch bestimmte Verhaltensweisen der Betroffenen gemindert oder abgewendet werden können. Fraglich ist hier, ob Kindern und Erwachsenen die gleichen oder zumindest ähnliche Abwehrmechanismen zur Verfügung stehen (U.S. EPA et al., 2003: 3-5).

Außerdem können gesundheitliche Beeinträchtigungen isoliert oder in Kombination mit weiteren Erkrankungen auftreten. Wenn ein Krankheitsbild mit weiteren Symptomen einhergeht, stellt sich die Frage, ob Kinder und Erwachsene in gleicher Weise von diesen zusätzlichen Gesundheitseffekten betroffen sind (U.S. EPA et al., 2003: 3-5). Weiterhin ist denkbar, dass eine Erkrankung ausschließlich bei Kindern oder Erwachsenen in Kombination mit weiteren Beeinträchtigungen auftritt. Da bei isoliert auftretenden Erkrankungen mit

\footnotetext{
${ }^{21}$ Eine erhöhte Bleikonzentration im Blut kann bei Kindern zu kognitiven Entwicklungsschäden führen, bei Erwachsenen hingegen zu Nierenproblemen oder Bluthochdruck.
} 
geringeren WTP zu rechnen ist als bei kombinatorischen, kann eine Vernachlässigung dieses Effekts zu verzerrten Ergebnissen führen (U.S. EPA et al., 2003: 3-5).

Das Vorliegen einer Latenzperiode zwischen Exposition mit einer Umweltkontamination und dem Auftreten einer Erkrankung bedarf weiterer Beachtung. Grundsätzlich wird die Reduzierung unmittelbarer Gesundheitsrisiken höher bewertet als die verzögert wirksam werdender Gesundheitsgefahren. Für den Fall, dass Unterschiede bezüglich der Latenzzeit zwischen Kindern und Erwachsenen bestehen, müssen diese bei der Entscheidung, ob eine Übertragung der WTP von Erwachsenen auf Kinder möglich ist, berücksichtigt werden (U.S. EPA et al., 2003: 3-5).

\subsubsection{Teilschritt 3: Bestimmung der Auswirkungen auf das Wohlergehen}

Der dritte Teilschritt bezieht sich auf die Beeinflussung des individuellen Wohlergehens durch gesundheitliche Beeinträchtigungen. Eine Erkrankung ist meist mit Schmerzen, einer bestimmten Anzahl an Krankheitstagen und Kosten für medizinische Behandlungen verbunden. Diese Größen bewirken eine Minderung der individuellen und sozialen Wohlfahrt. Sollen erwachsenenspezifische Daten auf Kinder übertragen werden, ist daher zu prüfen, ob diese Einflussfaktoren auch in Fällen einer Erkrankung von Kindern wirksam werden. Auch kann es weitere Faktoren geben, die Wohlfahrtseffekte bedingen. Eine chronische Erkrankung eines Kindes kann zum Beispiel zahlreiche Fehltage in der Schule herbeiführen, so dass sich die Berufsperspektive des betroffenen Kindes verschlechtert. Zusätzlich zu den genannten Einflussgrößen ist bei einer Erkrankung von Kindern noch mit weiteren Einflussgrößen zu rechnen, die sich auf die individuelle und soziale Wohlfahrt auswirken können. Hierzu gehört beispielsweise die Zeit, die Eltern in die Betreuung erkrankter Kinder investieren und die wiederum Fehltage der Eltern am Arbeitsplatz verursachen. Auch das Mitgefühl der Eltern und die Sorge um das erkrankte Kind, muss im Rahmen der Analyse berücksichtigt werden (U.S. EPA et al., 2003: 3-5f).

\subsubsection{Teilschritt 4: Beschreibung der Populationscharakteristika}

Der letzte Teilschritt der ersten Phase dient der Aufarbeitung des politischen Sachverhalts und beinhaltet eine Diskussion der demographischen Merkmale der betroffen Population und zwar in der Originalstudie, wie auch in dem neuen Bewertungszusammenhang. Demographische Merkmale wie Alter, Einkommen, Bildung und Gesundheitsstatus der Originalpopulation können die WTP für die Reduzierung eines umweltbedingten Gesundheitsrisikos beeinflussen. Gleiches gilt auch für die Kinder, auf die die Werte der Originalstudien übertragen werden sollen. Auch hier können Gesundheitszustand, Alter und Bildungsstand 
Auswirkungen auf die WTP für die Reduzierung von Gesundheitsrisiken haben und sind daher offen zu legen (U.S. EPA et al., 2003: 3-6).

\subsubsection{Phase 2: Beurteilung der Brauchbarkeit vorhandener Untersuchungen}

Für die Frage, ob die Ergebnisse einer bestimmten Untersuchung zur Übertragung auf den neuen Bewertungszusammenhang geeignet sind, sind zwei Aspekte von Bedeutung. Zum einen die Qualität der Originalstudie und zum anderen ihre Ähnlichkeit mit dem neuen Untersuchungsgebiet. Während sich die Studienqualität auf die Zuverlässigkeit der Bewertungsmethode und Validität der Ergebnisse bezieht, zielt die Studienähnlichkeit auf die Analogien zwischen der Originalstudie und dem neuen Untersuchungszusammenhang ab. Aufgrund der bereits dargestellten Risiko- und Bewertungsunterschiede muss eine Originalstudie, die den erwachsenorientierten Sachverhalt angemessen beschreibt, nicht zwangsläufig auch den kinderspezifischen Fall adäquat abbilden (U.S. EPA et al., 2003: 3-6).

\subsubsection{Zur Beurteilung der Studienqualität}

Zur Beurteilung der Studienqualität kann zunächst auf Kapitel 7 des „Guidlines for Preparing Economic Analyses“ (U.S. EPA, 2000) zurückgegriffen werden. Darüber hinaus werden im „Children's Health Valuation Handbook“ weitere Aspekte angeführt, welche bedeutsam sind, wenn die Qualität einer Studie getestet wird, deren Daten auf Kinder übertragen werden sollen (U.S. EPA et al., 2003: 3-6f). Hierzu gehören folgende Fragestellungen:

1. Folgt die Originalstudie der derzeit besten Forschungspraxis zur Schätzung von gesundheitsbezogenen WTP-Werten (für Kinder)?

Welches methodische Vorgehen die beste Alternative zur Erhebung von kinderspezifischer WTP für die Reduzierung von Gesundheitsrisiken darstellt, ist noch nicht abschließend geklärt. In Abschnitt 7.5 „Guidlines for Preparing Economic Analyses“ (U.S. EPA, 2000) sind jedoch zahlreiche Empfehlungen für die Erhebung erwachsenenorientierter WTP zu finden.

2. Wurde die Originaluntersuchung in einem peer-reviewed Journal veröffentlicht und wird sie häufig von Experten zitiert?

Grundsätzlich stellt die Veröffentlichung in einer begutachteten Zeitschrift einen zuverlässigen Anhaltspunkt für die Qualität einer Studie dar. Da die Anzahl kinderspezifischer Untersuchungen bisher aber sehr begrenzt ist, empfiehlt sich auch die Recherche nach Konferenzbeiträgen, Dissertationen oder bisher nicht veröffentlichen Manuskripten. Die Qualität dieses Materials sollte dann jedoch von unabhängigen Experten beurteilt werden (U.S. EPA et al., 2003: 3-7). 
3. Sind die Untersuchungsergebnisse konsistent mit den Ergebnissen anderer Studien?

Für den Fall, dass kinderspezifische Werte einer Studie erneut auf Kinder übertragen werden sollen, ist die Anzahl von Vergleichsstudien in der Regel gering. Unabhängig davon, ob kinder- oder erwachsenenbezogene Werte übertragen werden sollen, muss beim Abgleich der Ergebnisse darauf geachtet werden, dass die Untersuchungen auf die Messung der gleichen Gesundheits- und Wohlfahrtseffekte abzielen. Nur unter dieser Voraussetzung ist auch eine Vergleichbarkeit der Studien gegeben (U.S. EPA et al., 2003: 3-7).

4. Sind die Ergebnisse der Originalstudie konform mit relevanten Theoriekonzepten?

$\mathrm{Zu}$ prüfen ist hier, ob die theoretische Basis der Originalstudie auf fundierten ökonomischen Konzepten beruht und ob diese korrekt in der Studie umgesetzt worden sind (U.S. EPA et al., 2003: 3-7).

\subsubsection{Zur Beurteilung der Studienähnlichkeit}

Die Beurteilung der Kompatibilität unterschiedlicher Bewertungszusammenhänge erfolgt prinzipiell durch den Vergleich der Originalstudie mit den Charakteristika des neuen Untersuchungsgegenstands. Im Einzelnen beinhaltet dieser Vergleich die folgenden Fragestellungen (U.S. EPA et al., 2003: 3-8f):

1. Gibt es Gemeinsamkeiten zwischen der Originalpopulation und der Population des neuen Untersuchungsbereichs?

$\mathrm{Zu}$ prüfen ist hier, ob die demographischen Merkmale der Originalpopulation mit denen der neu zu untersuchenden Population übereinstimmen. Von besonderer Bedeutung bei der Übertragung erwachsenenbezogener Werte auf Kinder ist der sozioökonomische Status (U.S. EPA et al., 2003: 3-8).

2. Sind die Gesundheitseffekte im neuen Bewertungskontext vergleichbar mit denen, die in der Originalstudie erhoben wurden?

Negative Umwelteinflüsse können bei Kindern und Erwachsenen zu identischen physischen Reaktionen führen, jedoch mit unterschiedlichen Auswirkungen auf die Gesundheit. Daher ist unbedingt sicherzustellen, dass die gesundheitlichen Folgen eines bestimmten physischen Effekts bei Kindern und Erwachsenen zumindest annährend identisch $\operatorname{sind}^{22}$ (U.S. EPA et al., 2003: 3-8).

3. Ist die Messung der Wohlfahrtseffekte kompatibel mit der neu angelegten Studie?

\footnotetext{
${ }^{22}$ Ein Beispiel hierfür wäre eine erhöhte Bleikonzentration im Blut, die unterschiedliche Auswirkungen auf die Gesundheit von Kindern und Erwachsenen hat.
} 
Zu klären ist hier, inwieweit die Methode zur Erfassung der Wohlfahrtseffekte in der Originalstudie auch für die Messung von Wohlfahrtseffekten in einem kinderbezogenen Bewertungskontext geeignet ist. Ein verlorener Arbeitstag ist beispielsweise nicht zweckmäßig, um die Auswirkungen einer Erkrankung bei Kindern wieder zu geben.

4. Sind Originalstudie und neuer Bewertungskontext äquivalent hinsichtlich des Grundrisikos und des Ausmaßes der Risikoreduktion?

Im Allgemeinen wird von einem proportionalen Zusammenhang zwischen der WTP und dem Ausmaß der Risikoreduktion ausgegangen. Wenn der Gefährdungsgrad und das Maß der Risikoreduktion deutlich zwischen der Originalpopulation und der neu zu untersuchenden Population divergieren, muss über den Transfer der Nutzenfunktion die Änderung der WTP abgeleitet werden (U.S. EPA et al., 2003: 3-8).

5. Ähneln sich Originalstudie und neuer Untersuchungszusammenhang hinsichtlich der Schwere der zu erwartenden gesundheitlichen Beeinträchtigungen?

Auch wenn die gleichen Erkrankungen bei Kindern und Erwachsenen infolge eines negativen Umwelteinflusses auftreten, können Dauer, Schwere und Häufigkeit der Beeinträchtigungen differieren. Außerdem ist $\mathrm{zu}$ beachten, dass Kinder aufgrund der größeren Anzahl verleibender Lebensjahre länger unter chronischen Effekten leiden als Erwachsene. Mit chronischen Erkrankungen von Kindern können darüber hinaus indirekte Langzeiteffekte wie Bildungsdefizite einhergehen (U.S. EPA et al., 2003: 3-8).

6. Stehen der Population in der Originalstudie vergleichbare Abwehrmechanismen zur Reduzierung der Gesundheitsgefahr zur Verfügung wie den Personen im neuen Bewertungskontext?

Problematisch ist hier, dass Erwachsene zwar versuchen können das Verhalten eines Kindes zu kontrollieren, um Gesundheitsrisiken abzuwenden. Die Kinder müssen diese Verhaltensregeln aber auch befolgen und dazu in der Lage sein, sie umzusetzen. Nur dann stünden Kindern die gleichen Möglichkeiten zur Risikoabwendung zur Verfügung wie den Erwachsenen. Sind sich die Sorgeberechtigten bestimmten Gesundheitsgefahren nicht bewusst oder nehmen sie diese falsch wahr, können die erwachsenbezogenen Daten die kinderspezifischen Werte ebenfalls nicht exakt widerspiegeln (U.S. EPA et al., 2003: 3-9).

\subsubsection{Phase 3: ̈̈bertragung der Daten}

Jede Art des Nutzentransfers erfordert eine Anpassung der Daten aus der Originalstudie an den neuen Bewertungszusammenhang. Hierzu müssen die Originaldaten an die neue zu untersuchende Population angenährt werden und zwar unter Berücksichtigung der Risiko- und 
Bewertungsunterschiede. Für den Transfer von Daten auf einen kinderspezifischen Bewertungskontext kommen zwei Verfahren in Betracht: Zum einen die Übertragung von Punktschätzungen und zum anderen der Transfer von Nutzenfunktionen ${ }^{23}$ (U.S. EPA et al., 2003: 3-9f).

\subsubsection{Zur Übertragung von Punktschätzungen}

Wenn Werte aus erwachsenenorientierten Untersuchungen auf Kinder übertragen werden, kommt es selten vor, dass Originalstudie und neuer Bewertungszusammenhang in Bezug auf alle Parameter identisch sind. Daher müssen punktgenaue WTP-Schätzungen an die neue Population angeglichen werden. Diese Anpassung der Werte muss unter Bezugnahme der Bewertungs- und Risikounterschiede zwischen Kindern und Erwachsenen erfolgen. Für die Berücksichtigung des höheren Wohlstandniveaus von Kindern im Vergleich zur jetzigen Erwachsenengeneration wird im „Children's Health Valuation Handbook“ eine Anpassungsmethode beschrieben. Auf dieses Verfahren wird nachstehend eingegangen. Alle weiteren Anpassungen erwachsenenbezogener Werte an die Merkmale der Kinderpopulation bedürfen einer detaillierten Aufschlüsslung und Begründung (U.S. EPA et al., 2003: 3-9f).

\subsubsection{Zum Transfer von Nutzenfunktionen}

Die Übertragung von Nutzenfunktionen ist im Vergleich zur Übernahme von Punktschätzungen komplizierter, bietet aber auch umfassendere Möglichkeiten zur Anpassung der Originaldaten an die neue Population. Wird eine Zahlungsbereitschaftsfunktion übernommen, können spezifische Größen der neuen Population (wie das Ausgangsrisiko für eine Erkrankung) die Originalwerte in der Funktion ersetzten.

Kritisch anzumerken ist jedoch, dass die Reliabilität des Transfers von Nutzenfunktionen auch von der Übereinstimmung der Koeffizienten der jeweiligen Nutzenfunktionen der Originalstudie und den Parametern des neuen Bewertungskontextes abhängt. Aufgrund der bereits angeführten Risiko- und Bewertungsunterschiede zwischen Kindern und Erwachsenen ist eine weitgehende Übereinstimmung jedoch eher unwahrscheinlich. Altruistische Motive beispielsweise können in einen kinderspezifischen Bewertungskontext einen signifikanten Einfluss auf die Höhe der WTP ausüben. Für die Bewertung der Gesundheit eines Erwachsenen ist diese Größe dagegen weniger von Bedeutung. Werden relevante

\footnotetext{
${ }^{23}$ Prinzipiell kommt als dritte Methode auch noch ein metaanalytisches Verfahren in Betracht. Dieses ist als Transferansatz geeignet, wenn Werte aus mehreren Studien zur Verfügung stehen und zusammengeführt werden sollen. Da im Bereich der Bewertung von Kindergesundheit jedoch die Anzahl der Studien sehr begrenzt ist, wird auf die Metaanalyse im „Children’s Health Valuation Handbook“ nicht weiter eingegangen.
} 
Einflussfaktoren der kinderspezifischen WTP bei der Übertragung von Nutzenfunktionen ignoriert, führt dies zu Verzerrungen (U.S. EPA et al., 2003: 3-10).

\subsubsection{Annährung aktueller WTP an ein zukünftiges Wohlstandsniveau}

Generell wird davon ausgegangen, dass der gesellschaftliche Wohlstand stetig zunimmt. Im „Children’s Health Valuation Handbook“ wird ein Verfahren beschrieben, mit dem die WTP aus einer erwachsenenorientierten Originalstudie an das erwartende Wohlstandsniveau der Kinder im späteren Erwachsenalter angepasst werden kann ${ }^{24}$.

Abbildung 2 zeigt die Gleichung, mit der die WTP der aktuellen Erwachsenengeneration für die Reduzierung eines Gesundheitsrisikos in eine zukünftige erwachsenorientierte WTP überführt werden kann und zwar unter Berücksichtigung des steigenden Wohlstandsniveaus einer Gesellschaft (U.S. EPA et al., 2003: 3-10f).

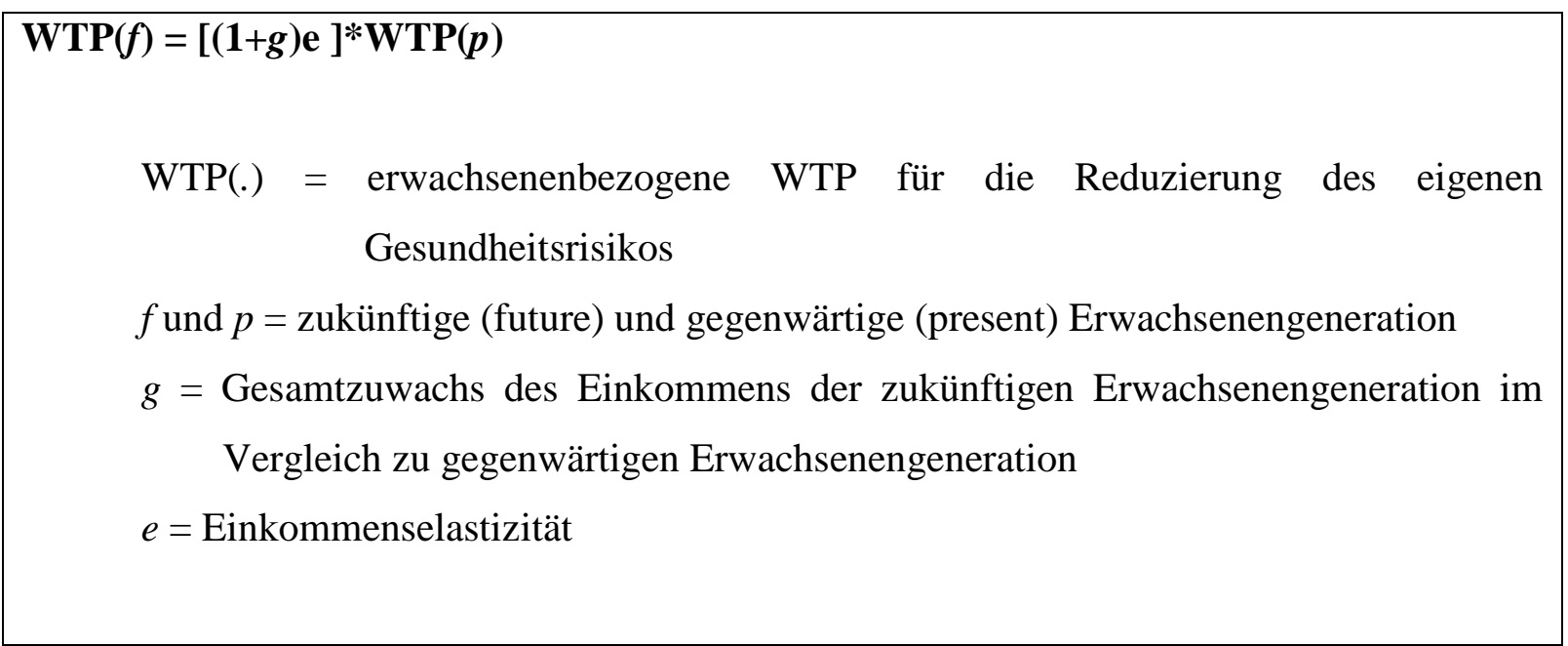

Abbildung 2: Anpassung des Wohlstandsniveaus der aktuellen Erwachsenengeneration an zukünftige Erwachsenengenerationen (U.S. EPA et al., 2003: 3-11)

Die Anwendung dieser Gleichung auf die erwachsenenbezogenen WTP bewirkt eine Annährung an den kinderorientierten Bewertungszusammenhang und führt damit zu solideren Schätzungen der ökonomischen Vorteilhaftigkeit politischer Interventionen (U.S. EPA et al., 2003: 3-11).

\subsubsection{Phase 4: Qualitative Beschreibung von Unsicherheiten}

Die Übertragung erwachsenenbezogener WTP auf Kinder ist, wie dargestellt, mit zahlreichen Unsicherheiten verbunden. Die große Anzahl möglicher Fehlerquellen beruht in erster Linie auf den genannten Risiko- und Bewertungsunterschieden zwischen Kindern und Erwachsenen. Aus diesem Grund stellt der Transfer erwachsenenbezogener Daten auf Kinder

\footnotetext{
${ }^{24}$ Dieses Verfahren muss nur im Rahmen der Werteübertragung von Erwachsenen auf Kinder angewandt werden. Wird die WTP für die Reduzierung eines Gesundheitsrisikos über die Eltern erhoben ist einen Datenanpassung nicht notwendig (U.S. EPA et al., 2003: 3-10).
} 
auch nur eine Annährung an die „wahre“ WTP für die Reduzierung umweltbedingter Gesundheitsrisiken für Kinder dar. Die übertragenen und gegebenenfalls an den neuen Bewertungskontext angepassten Werte können sowohl nach oben als auch nach unten von den eigentlichen kinderspezifischen WTP abweichen. Wichtig ist jedoch, die möglichen Gründe für Verzerrungen der WTP zu diskutieren und anzugeben, ob eher Über- oder Unterschätzungen der Werte $\mathrm{zu}$ erwarten sind. Darüber hinaus sollten auch die Übertragungstechnik an sich kritisch betrachtet und eventuelle Grenzen und Fehlerquellen der Methode offen gelegt werden (U.S. EPA et al., 2003: 3-11f).

\subsubsection{Zwischenfazit}

Die Übertragung von erwachsenenorientierter WTP zur Reduzierung eines umweltbedingten Gesundheitsrisikos auf Kinder ist aufgrund des Mangels an kinderspezifischen Untersuchungen notwendig. Allerdings ist der Transferprozess mit vielen Untersicherheiten verbunden, welche auf Risiko- und Bewertungsunterschieden zwischen Kindern und Erwachsenen beruhen. Wird die Nutzenkomponente im Rahmen einer Kosten-NutzenAnalyse über Transfermethoden ermittelt, dürfen Aussagen zur Effizienz politischer Maßnahmen nur als grobe Schätzungen interpretiert werden.

In Fällen, wo ungefähre Annährungen an die Wirksamkeit umwelt- oder gesundheitspolitischer Maßnahmen zum Schutz von Kindergesundheit nicht ausreichen, sollte eine spezifische WTP für Kinder ermittelt werden. Zu beachten ist hier jedoch, dass auch die direkte Erhebung einer WTP für die Reduzierung eines Gesundheitsrisikos für Kinder mit Unsicherheiten und Verzerrungen einhergehen kann. Grundsätzlich kann empfohlen werden, die Unsicherheiten und möglichen Fehlerquellen einer direkten Erhebung kinderspezifischer WTP gegen die eines Nutzentransfers abzuwiegen und auf Basis dieses Vergleichs eine Entscheidung für eine der genannten Vorgehensweisen zu treffen (U.S. EPA et al., 2003: 312).

\subsection{Zur Zusammenarbeit von Risikobewertern und Ökonomen}

Abschließend wird im „Children’s Health Valuation Handbook“ auf die Wichtigkeit einer kontinuierlichen Zusammenarbeit von Risikobewertern und Ökonomen hingewiesen. Die Untersuchung der Wirtschaftlichkeit einer umweltpolitischen Maßnahme zur Reduzierung von Gesundheitsrisiken erfordert die Offenlegung verschiedener Aspekte, die die bestehende Gefährdungssituation kennzeichnen. Diese Risikocharakterisierung wird in der Regel von Risikobewertern übernommen. Ein frühzeitiger Informationsaustausch zwischen den 
Akteuren soll jedoch gewährleisten, dass im Rahmen der Risikoabschätzung auch die Daten bereitgestellt werden, welche für die ökonomische Analyse politischer Intervention benötigt werden. Diesbezüglich wird im „Children’s Health Valuation Handbook“ erläutert, welche Fragestellungen für eine zielgerichtete Kommunikation zwischen Risikobewertern und Ökonomen von Bedeutung sind. Hierzu gehört einerseits eine Abstimmung über die zu erwartenden umweltbedingten Gesundheitseffekte und deren Erfassung. Andererseits aber auch der Austausch über Ausmaß der aktuellen Umweltbelastung und eine Prognose über zukünftige Änderungen der Belastungsstärke.

Des Weiteren sollten Ökonomen über die soziodemographischen Merkmale und die Altersverteilung der gefährdeten Population aufgeklärt werden. Schließlich sind die Unsicherheiten, welche mit dem Prozess der Risikoevaluierung verbunden sind, gemeinsam von Risikobewertern und Ökonomen zu diskutieren und wenn möglich zu quantifizieren (U.S. EPA ET AL., 2003: 6-4f). Zusätzlich wird die Einbeziehung externer Experten, beispielsweise aus der Industrie, empfohlen, um eventuell auftretende Unsicherheit bei der Abschätzung von Gesundheitsrisiken und Wohlfahrtseffekten zu beheben (U.S. EPA et al., 2003: 6-3).

\subsection{Abschließende Bemerkung zum „Children’s Health Valuation Handbook“}

Das „Children’s Health Valuation Handbook“ liefert einen kompakten Überblick über die besonderen Herausforderungen, welche mit der Ermittlung des kinderspezifischen Nutzens umweltpolitischer Maßnahmen zum Schutz von Kindergesundheit verbunden sind. Grundsätzlich sollte die Ermittlung des Nutzens entsprechend des „Children's Health Valuation Handbook“ über die Bestimmung der WTP erfolgen. Diesbezüglich werden verschiedene Methoden zu Erfassung der WTP dargestellt, wovon letztlich für die Erhebung der kinderspezifischen Präferenzen nur der „Stated Preferences“ Ansatz geeignet erscheint.

Wie eine Befragung $\mathrm{zu}$ umweltpolitischen Gesundheitsprojekten für Kinder konkret umgesetzt werden kann, bleibt im „Children’s Health Valuation Handbook“ aber offen. Klar herausgestellt wird indes, dass (kleine) Kinder nicht direkt nach ihren Präferenzen in Bezug auf die Gesundheit befragt werden können. Ergänzende Hinweise auf weiterführende Literatur zur empirischen Erhebung von kinderspezifischer WTP über Stellvertreter sowie die damit verbundenen Herausforderungen werden dennoch nur begrenzt angeführt. Zwar wird auf einige konzeptionelle Probleme eingegangen, welche mit der direkten Erhebung kinderspezifischer WTP einhergehen können, wie beispielsweise die Wahl der Perspektive. Insgesamt sind die Informationen im „Children’s Health Valuation Handbook“ jedoch eher 
allgemein und für die praktische Umsetzung von Zahlungsbereitschaftsanalysen nur begrenzt hilfreich.

Positiv hervorzuheben ist die ausführliche Darstellung der Risiko- und Bewertungsunterschiede zwischen Kindern und Erwachsenen. Die genannten Unterschiede führen einerseits dazu, dass für die ökonomische Bewertung der Gesundheit von Kindern und Erwachsenen jeweils eigenständige Betrachtungen notwendig sind. Andererseits sind die genannten Differenzen für die Übertragung von WTP- und zum Teil auch COI-Werten von Erwachsenen auf Kinder (Benefit Transfer) bedeutsam.

$\mathrm{Da}$ es aus verschiedenen Gründen oft nicht möglich ist, kinderspezifische Daten zu erheben, sind für die Ermittlung des Nutzens eines umweltpolitischen Gesundheitsprogramms die „Checklisten“ zum Benefit Transfer nützlich. Die detaillierte und klar strukturierte Darstellung ermöglicht eine gute Nachvollziehbarkeit und eine direkte Anwendung in der Praxis. Fraglich ist jedoch, warum der Übertragung von Werten aus anderen Studien so viel Aufmerksamkeit entgegengebracht wird. In Anbetracht der zahlreichen Verzerrungen, welche aus dem Transfer der Werte von Erwachsen auf Kinder resultieren können, sollte die gesonderte Erfassung kinderspezifischer WTP und COI stärker im Vordergrund stehen.

Unklar ist auch, warum entsprechend des „Children’s Health Valuation Handbook“ der Nutzen eines Gesundheitsprojekts entweder über die WTP- oder die COI-Methode erfolgen soll. Berücksichtigt man auch die gesundheitsökonomische Literatur, müsste sowohl die WTP als auch die COI zur Bestimmung des gesamten Nutzens eines Gesundheitsprojekts ermittelt werden. Zudem ist die COI-Methode zur alleinigen Bestimmung des Nutzens eines umweltpolitischen Gesundheitsprojekts generell ungeeignet, wenn Aussagen über eine potentielle Pareto-Verbesserung getroffen werden sollen. Schließlich können über die Kalkulation der COI keine immateriellen Effekte berücksichtigt werden, so dass auch keine Rückschlüsse auf eine Wohlfahrtsänderung möglich sind.

Insgesamt liefert das „Children’s Health Valuation Handbook“ aber einige nützliche Hinweise und zeigt unterschiedliche Probleme bei der Durchführung ökonomischer Bewertungen umweltpolitischer Programme zum Schutz von Kindergesundheit auf. In Bezug auf konkrete Probleme und eine praktische Umsetzung von Kosten-Nutzen-Analysen zur Bewertung von Kindergesundheit werden jedoch kaum Lösungen präsentiert. Stattdessen wird mehrfach auf die allgemeinen Empfehlungen des „Guidelines for Preparing Economic Analysis“ verwiesen. Zudem wird auf die in der wissenschaftlichen Literatur häufig angeführten konzeptionellen und methodischen Probleme, wie der Einfluss altruistischer Motive, die Auswahl eines 
Haushaltsmodells oder Effekte sozialer Erwünschtheit, gar nicht oder nur kurz eingegangen.

Dadurch bleibt unklar, wie auf diese Herausforderungen zu reagieren ist.

Insgesamt eignet sich das „Children's Health Valuation Handbook“ als Einstig in den Themenkomplex „Ökonomische Bewertungen“ in Bezug auf „Kindergesundheit und Umweltgefahren“. Letztlich wird es damit dem Anspruch einen informativen Charakter zu besitzen gerecht, liefert für den informierten Leser aber nur begrenzten Erkenntnisgewinn.

\section{Vergleich von PEPa und „Children's Health Valuation Handbook“6}

Das „Children’s Health Valuation Handbook“ und das PEPa zeigen Möglichkeiten auf, um umweltpolitische Maßnahmen zum Schutz von Kindergesundheit ökonomisch zu bewerten. In beiden Dokumenten wird die Kosten-Nutzen-Analyse als bevorzugte Bewertungsmethode benannt. Begründet wird diese Empfehlung damit, dass nur die Kosten-Nutzen-Analyse Rückschlüsse auf die ökonomische Effizienz ermöglicht. Das PEPa stellt die Ermittlung der Kosten, die durch verkehrsbedingte Erkrankungen verursacht werden, in den Vordergrund. Das „Children’s Health Valuation Handbook“ konzentriert sich hingegen auf die Erfassung des Nutzens infolge der Reduzierung umweltbedingter Erkrankungen.

Gemäß beiden Dokumenten sollen der (immaterielle) Nutzen /die immateriellen Kosten von Kindergesundheitsprojekten berücksichtigt werden. Zur Messung der WTP wird die „Stated Preferences“ Methode empfohlen in Übereinstimmung mit der aktuellen wissenschaftlichen Literatur. Dabei besteht Einigkeit darüber, dass Kinder nicht selbst sondern deren Eltern nach einer WTP befragt werden müssen. Bezüglich der Frage, ab welchem Alter die Präferenzen von Kindern im Rahmen ökonomischer Evaluationen berücksichtigt werden sollten, werden jedoch keine weiterführenden Empfehlungen formuliert.

Im PEPa wird für die Kalkulation der umweltbedingten direkten Kosten zusätzlich auf den COI-Ansatz verwiesen entsprechend gesundheitsökonomischer Ansätze. Das „Children’s Health Valuation Handbook" schlägt dagegen vor, die COI alternativ zu bestimmen, wenn die Erhebung der WTP nicht möglich ist. Dadurch würden jedoch die immateriellen Effekte nicht erfasst, was zu einer Unterschätzung des volkswirtschaftlichen Nutzens eines Kindergesundheitsprojekts führen würde.

Aus beiden Dokumenten geht übereinstimmend hervor, dass die praktische Umsetzung von Kosten-Nutzen-Analysen zur Bewertung von Kindergesundheit noch mit vielen Unsicherheiten behaftet ist. Gründe dafür sind Schwierigkeiten bei der Erfassung kinderspezifischer ökonomischer Kennzahlen sowie die unzureichende Verfügbarkeit spezieller epidemiologischer Daten. 
Aufgrund dieses Datenmangels werden im „Children’s Health Valuation Handbook“ und im PEPa Möglichkeiten einer Übertragung von erwachsenenbezogenen Werten auf Kinder diskutiert. In diesem Kontext wird im „Children’s Health Valuation Handbook“ intensiv auf den Transfer der WTP von Erwachsenen auf Kinder eingegangen. Dagegen wird im PEPa die potentielle Nutzung erwachenenbezogener Ursache-Wirkungs-Beziehungen in kinderspezifischen Analysen erörtert.

Letztlich ist die Verwendung erwachsenenbezogener Daten in kinderspezifischen Bewertungszusammenhängen aber kritisch zu beurteilen. Die Ergebnisse solcher Analysen dürfen höchstens als grobe Annährungen an das „wahre“ Nutzen-Kosten-Verhältnis verstanden werden. Grund dafür sind die erheblichen Risiko- und Bewertungsunterschiede zwischen Kindern und Erwachsenen, die in beiden Dokumenten ausführlich thematisiert werden. Infolge dieser Unterschiede können Kosten und Nutzen aus erwachsenenbezogen Untersuchungen weder die höhere Gesundheitsgefährdung von Kindern durch Umweltrisiken noch die besondere Wertschätzung von Kindergesundheit angemessen berücksichtigen.

Eine andere Möglichkeit, dem Problem fehlender Werte bei Kosten-Nutzen-Analysen zu begegnen, ist der Rückgriff auf alternative Bewertungsmethoden. Im „Children’s Health Valuation Handbook“ werden hierfür verschiedene Ansätze vorgestellt. Diese Verfahren benötigen zur Bewertung eines Programms entweder nur Daten zum Erkrankungsrisiko von Kindern oder die WTP. Der Nachteil dieser Methoden ist jedoch, dass sie keine Messung der ökonomischen Effizienz eines Kindergesundheitsprojekts erlauben. Unabhängig davon, kann die Anwendung zusätzlicher Bewertungsverfahren aber der Absicherung der Ergebnisse einer Kosten-Nutzen-Analyse dienen.

Fraglich ist, warum im „Children’s Health Valuation Handbook“ und auch im PEPa kaum explizit auf die praktische Durchführung von Zahlungsbereitschaftsanalysen eingegangen wird. Die besonderen methodischen und konzeptionellen Anforderungen, die die Erhebung kinderbezogener WTP an den Forscher stellt, werden weder im PEPa noch im „Children’s Health Valuation Handbook“ weiter ausgeführt. Die vielfach in der Wissenschaft diskutierten Quellen für mögliche Verzerrungen der Daten bleiben außen vor. Im „Children’s Health Valuation Handbook" wird lediglich kurz auf den Einfluss altruistischer Motive eingegangen, jedoch ohne Lösungsansätze aufzuzeigen. Somit bleibt unklar, wie mit den verschiedenen Bewertungsproblemen umzugehen ist. Um kinderbezogene Studien durchführen zu können, sollten die Dokumente detaillierter auf die spezifischen Probleme eingehen, die hiermit verbunden sind. 
Hilfreich für die Erhebung kinderbezogener WTP wären praxisbezogene Hinweise zum Untersuchungsdesign und ein Leitfaden für die Konstruktion eines zuverlässigen Erhebungsinstruments. Denkbar wäre in diesem Zusammenhang auch die Entwicklung einer Itembatterie, welche hinsichtlich ihrer Messgüte ausreichend getestet ist. Hieraus könnten dann jeweils zumindest einige geeignete Fragen für den konkreten Bewertungszusammenhang übernommen werden. Dies würde die Erhebung kinderspezifischer WTP in der Praxis zum einen vereinfachen und zum anderen die Datenqualität verbessern. Schließlich wären auch detaillierte Empfehlungen zur Bestimmung der direkten und indirekten Kosten- und Nutzeneffekte wünschenswert, um die Durchführung der Kosten-Nutzen-Analysen zu erleichtern. Auf diese Weise könnte die Akzeptanz von ökonomischen Bewertungsverfahren in der Umweltpolitik insgesamt gesteigert werden.

\section{$9 \quad$ Abschließende Bemerkung}

Die Reduzierung gesundheitsschädlicher Umwelteinflüsse zählt mittlerweile zu den zentralen Aufgaben der internationalen Umweltpolitik. Besondere Aufmerksamkeit gilt dabei dem Schutz von Kindergesundheit, da Kinder besonders sensibel auf negative Umwelteinflüsse reagieren.

In Anbetracht knapper finanzieller Ressourcen und kontinuierlich steigender Ausgaben für die Erhaltung der öffentlichen Gesundheit ist aktuell nicht mehr nur die Wirksamkeit sondern auch die Wirtschaftlichkeit umweltpolitischer Intervention von großer Bedeutung. Vor diesem Hintergrund ist ein steigendes Interesse politischer Entscheidungsträger an ökonomischen Bewertungsverfahren, insbesondere der Kosten-Nutzen-Analyse, zu beobachten.

Bisher wurden Kosten-Nutzen-Analysen vor allem in der Wissenschaft und im Bereich allgemeiner Umweltbewertungen, zum Beispiel im Naturschutz eingesetzt. Die aktuelle Entwicklung in der Umweltpolitik lässt aber darauf schließen, dass die Kosten-NutzenAnalyse zukünftig auch zum Einsatz kommen wird, um die Effizienz von Kindergesundheitsprojekten zu prüfen. Beispielhaft hierfür sind die Konzeption eines Leitfadens zur Durchführung von Kosten-Nutzen-Analysen im Rahmen des PEP und PEPa sowie die Formulierung des „Children’s Health Valuation Handbook“.

Auf theoretischer Ebene haben sich Ökonomen über die wesentlichen Inhalte der monetären Bewertung von Gesundheitsprogrammen verständigt. So ist der volkswirtschaftliche Nutzen definiert als „maximale Zahlungsbereitschaft der Projektbefürworter“. Unter dem volkswirtschaftlichen Kosten werden die ,minimalen Entschädigungsforderungen der Projektgegner“ verstanden. Für eine Kosten-Nutzen-Analyse sind direkte und indirekte 
materielle sowie immaterielle Kosten- und Nutzenkomponenten relevant. Die Ermittlung der materiellen und immateriellen Effekte eines umweltpolitischen Gesundheitsprojekts kann über direkte und indirekte Bewertungsmethoden erfolgen. Abbildung 3 zeigt, wie der volkswirtschaftliche Nutzen eines Programms zum Schutz von Gesundheit erhoben werden kann.

Ein in der Wissenschaft viel diskutierter Punkt in Verbindung mit kinderbezogenen KostenNutzen-Analysen ist, dass Kinder nicht direkt nach einer WTP für die Erhaltung ihrer Gesundheit befragt werden können. Stattdessen muss auf die elterliche WTP zurückgegriffen werden. Die Erhebung einer WTP über Stellvertreter kann jedoch zu Verzerrungen der Daten führen. Verantwortlich hierfür können unter anderem Effekte sozialer Erwünschtheit, der Einfluss altruistischer Motive aber auch die Unterstellung eines unpassenden Haushaltsmodells sein.

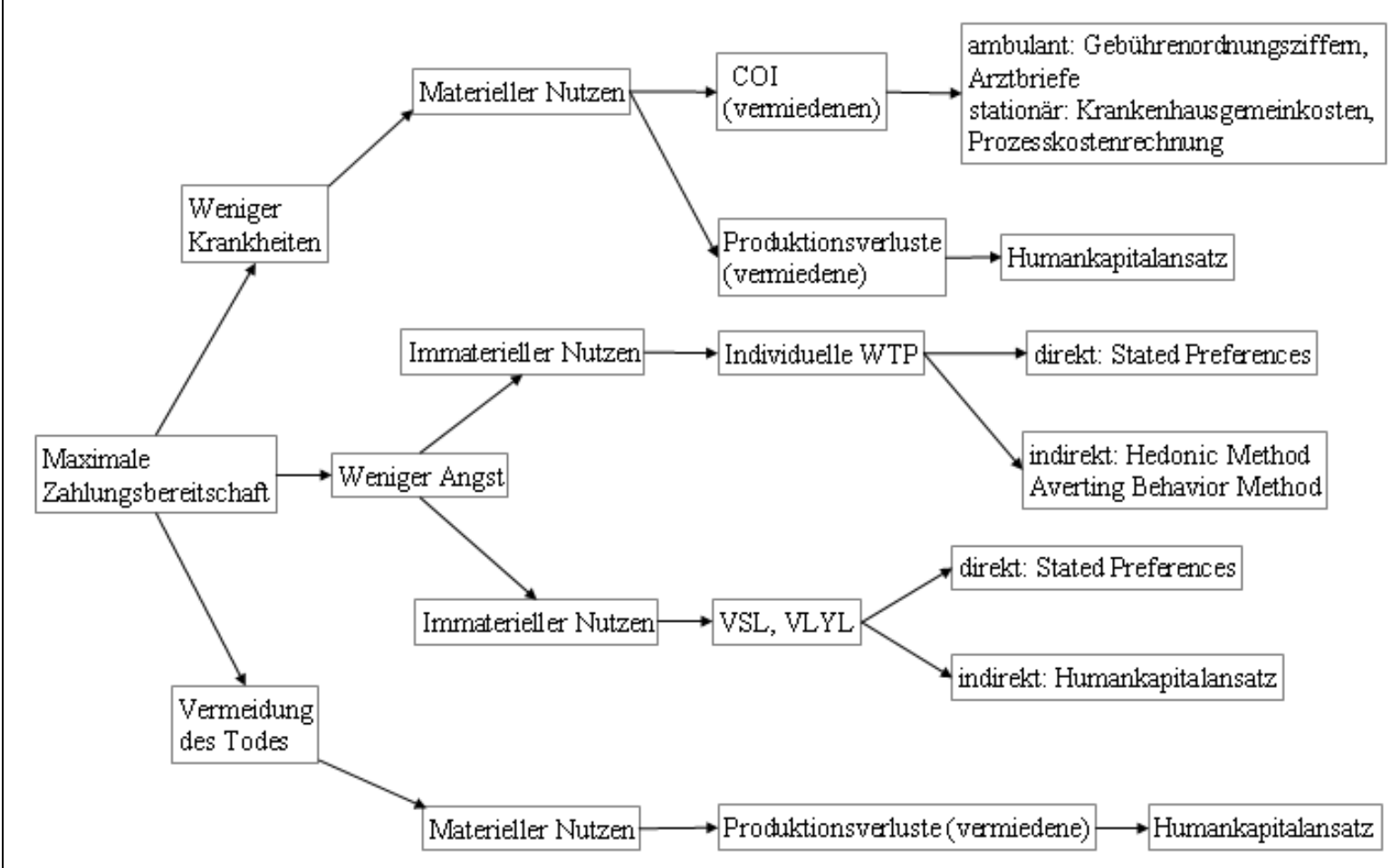

Abbildung 3: Methoden zur Bestimmung des volkswirtschaftlichen Nutzens umweltpolitischer Kinderschutzprojekte (eigene Darstellung)

Die Aufarbeitung der politischen Empfehlung zur ökonomischen Bewertung von Kindergesundheitsprojekten zeigt, dass es hinsichtlich der praktischen Durchführung von Kosten-Nutzen-Analysen noch weiterer Abstimmung bedarf. Eine besondere Herausforderung bei der ökonomischen Bewertung von Kindergesundheitsprojekten stellt die Tatsache dar, dass häufig keine eindeutigen Aussagen über die Ursache-WirkungsBeziehungen zwischen Umweltkontaminationen und dem Erkrankungsrisiko von Kindern 
getroffen werden können. Dadurch ist es einerseits unmöglich, die Krankheitskosten beziehungsweise deren Einsparungen durch ein umweltpolitisches Projekt klar zu beziffern. Andererseits ist es schwierig, eindeutige Aussagen über das Erkrankungsrisiko von Kindern durch bestimmte Umwelteinflüsse zu treffen. Des Weiteren ist auf methodischer und konzeptioneller Seite nicht geklärt, wie mögliche Verzerrungen, verursacht durch die Erhebung einer kinderbezogenen WTP über die Eltern, reduziert beziehungsweise kontrolliert werden können.

Somit stellen auch die aktuellen umweltpolitischen Empfehlungen zur Durchführung ökonomischer Bewertungen von Kindergesundheit, wie das PEPa oder das „Children’s Health Valuation Handbook“, keine gebrauchsfähigen Modelle zur Realisierung kinderbezogener Kosten-Nutzen-Analysen bereit. Für die bestehenden Bewertungsprobleme stellen auch sie keine zufriedenstellenden Lösungen bereit. Dies hat zur Folge, dass auf die spezifischen Herausforderungen, die mit der Umsetzung von kinderbezogenen Kosten-Nutzen-Analysen verbunden sind, in der Praxis noch unterschiedlich reagiert wird. Verschiedene Vorgehensweisen können aber $\mathrm{zu}$ abweichenden Ergebnissen für den gleichen Bewertungszusammenhang führen. Entsprechend ist die Reliabilität von Kosten-NutzenAnalysen für Kindergesundheitsprojekte derzeit noch gering. Dies erschwert die Berücksichtigung ökonomischer Bewertungen in der Politik.

Vor diesem Hintergrund muss es zukünftig darum gehen, einheitliche Durchführungsstandards für die praktische Umsetzung ökonomischer Bewertungen von Kindergesundheitsprojekten zu entwickeln. So besteht insgesamt noch Forschungs- und Diskussionsbedarf, um die Qualität und damit die Aussagekraft von Kosten-Nutzen-Analysen für den Bereich „Umweltpolitik und Kindergesundheit“ zu verbessern. Zum einen wird es Aufgabe der Medizin sein, die Lücken in Bezug auf epidemiologische Daten zur Kindergesundheit und $\mathrm{zu}$ Umweltkontaminationen $\mathrm{zu}$ schließen. Zum anderen ist auf ökonomischer Seite weitere Forschungsarbeit nötig, damit die beschriebenen Probleme bei der Erhebung einer kinderspezifischen WTP gelöst werden können.

Bis zur Abstimmung darüber, wie den Anforderungen, die kinderbezogene Kosten-NutzenAnalysen an den Forscher stellen, in der Praxis zu begegnen ist, dürfen die Untersuchungsergebnisse nur als grobe Schätzungen der Effizienz interpretiert werden. Trotzdem sollten ökonomische Bewertungen im Rahmen politischer Entscheidungsfindung Beachtung finden, um Anhaltspunkte für das Kosten-Nutzen-Verhältnis von Kindergesundheitsprojekten zu bekommen. Darüber hinaus könnte die WTP als 
präferenzbasiertes Nutzenmaß Aufschluss über das generelle Interesse der Bürger an einer politischen Maßnahme geben. 


\section{Literatur}

Agee, M. D. und Crocker, T. D. (1996): Parents' Discount Rates for Child Quality. Southern Economic Journal, 63: 36-50.

Alberini, A. und ChIABAI, A. (2006): Urban environmental health and sensitive populations: How much are the Italiens willing to pay to reduce their risks? Regional Science and Urban Economics, 37: 239-258.

Banasiak, U.; Heseker, H.; Sieke, C.; SOMmerfeld C. und Vohmann, C. (2005): Abschätzung der Aufnahme von Pflanzenschutzmittel-Rückständen in der Nahrung mit neuen Verzehrmengen

für Kinder. Bundesgesundheitsblatt-Bundesgesundheitforschung-Bundesgesundheitschutz, 48: 84-98.

BAteman, I. J. und MunRo, A. (2006): Household versus individual valuation: What's the difference? http://www.oecd.org/dataoecd/1/29/37585613.pdf zugegriffen am 15.8.2009.

Bergstrom, T. (1982): When is a Men's Worth More Than His Human Capital? Department of Econimics, University of California, Santa Barbara. Paper No. 1982d.

BlomQuist, G. C. (2003): Self Protection and Averting Behaviour, Values of Statistical Lives, and Benefit Cost Analysis of Environmental Policy. National Center for Environmental Economics, Working Paper No. 03-02, March.

BLOMQUIST, G. C. (1982): Estimating the Value of Life and Safety: Recent Developments. In: JonesLee, M.: The Value of Life and Safety. North-Holland Publishing Co., Amsterdam.

Boesch, H.-J.; Kahlmeier, S.; Sommer, H.; von Kempen, E.; StaAtsen, B. und Racioppi, F. (2008): Economic Valuation of transport-related health effects. Review of methods and development of practical approaches, with a special focus on children. World Health Organization (WHO) Regional Office for Europe, Denmark.

Brandes, W.; Recke, G. und Berger, T. (2001): Produktions- und Umweltökonomie. Band I. Verlag Eugen Ulmer, Stuttgart.

BREYER, F. und ZwEIFEL, P. (1999): Gesundheitsökonomie. Springer, Berlin Heidelberg New York. Bundeshaushaltsordnung, Bundesministerium der Justiz. Geltung ab 14.7.1990; zuletzt geändert am 29.07.2009. http://bundesrecht.juris.de/bundesrecht/bho/gesamt.pdf zugegriffen am 17.7. 2009.

Chamley, G. und Putzrath, R. M. (2001): Children's Health, Susceptibility and Regulatory Approaches to Reducing Risks from Chemical Carcinogens. Environmental Health Perspectives, 109 (2): 187-192. 
Dickie, M. und Gerking, S. (2006): “Valuing Children's Health: Parental Perspective”. In: Economic Valuation of Environmental Health Risks to Children. OECD: Paris.

DubourG, W. R.; Jones-LeE, M. W. und LOOMES, G. (1994): Imprecise preferences and the WTP WTA disparity. Journal of Risk and Uncertainty, 9: 115-133.

Ecoplan, Infras (2008): Externe Kosten des Verkehrs. Aufdatierung für das Jahr 2005 mit Bandbreiten. Berne, Ecoplan.

EsSER, H. (1986): Können Befragte Lügen? Zum Konzept des „wahren Wertes“ im Rahmen der handlungstheoretischen Erklärung von Situationseinflüssen bei der Befragung. In: Kölner Zeitschrift für Soziologie und Sozialpsychologie, 38: 314-336.

Executive Order 13045-Protection of Children From Environmental Health Risks and Safety Risks. Vom 23.04.1997. http://frwebgate.access.gpo.gov/cgi-bin/getdoc.cgi?dbname=1997_register $\&$ docid=fr23ap97-130.pdf zugegriffen am 14.08.2009.

FAden, R. und BeAuchamp, T. (1996) A History and Theory of Informed Consent, Oxford University Press, Oxford, U.K.

Fischhoff, B.; Lictenstein, S.; Read, S.und Combs, B. (1978): How Safe is Safe Enough? A Psychometric Study of Attitudes Toward Technological Risks and Benefits. Policy Sciences, 9: $127-152$.

GrEINER, W. (2007): Die Berechnung von Kosten und Nutzen. In: Schöffski, O.; Schulenburg J.-M. [Hrsg.]: Gesundheitsökonomische Evaluationen, Springer, Berlin.

HammitT, J. K. und GRAham, J. (1999): Willingness to Pay for Health Protection: Inadequate Sensitivity to Probability? Journal of Risk and Uncertainty, 18: 33-62.

Harbaugh, W. T.; Krause, K. und Vesterlund, L. (2002): Risk Attitudes of Children and Adults: Choices Over Small and Large Probability Gains and Losses. Experimental Economics, 5: $53-84$.

HoffmanN, S. (2007): Since Children are not little adults - socially - What's an environmental economist to do? Duke Environmental Law \& Policy Forum.

JENKIns, R. R.; OWEns, N. und Wiggins, L. B. (2001): Valuing Reduced Risks to Children: The Case of Bicycle Safety Helmets, Contemporary Economic Policy.19 (4): 397-408.

Johannesson, M. und Johansson, P.-O. (1996): To Be or Not Be, That is the Question: An Empirical Study on the WTP for an Increased Life Expectancy at an Advanced Age. Journal of Risk and Uncertainty, 13: 163-174.

Johannesson, M. und JohAnsson, P-O. (1997): Quality of Life and the WTP for An Increased Life Expectancy at an Advanced Age. Journal of Public Economics, 65: 219-228.

KLOSE, T. (1999): The contingent valuation method in health care. Healthy Policy, 47: 97-123. 
Kommission der Europäischen Gemeinschaft: Mitteilung der Kommission an den Rat, das Europäische Parlament und den Europäischen Wirtschafts- und Sozialausschuss: Eine europäische Strategie für Umwelt und Gesundheit. Brüssel, den 11.06.2003, KOM (2003) 338 endgültig.

LiU, J.-T.; HammitT, J .K.; WAnG, J.-D. und LiU, J.-L. (2000): Mother's Willingness to Pay for Her Own and Her Child's Health: A Contingent Valuation Study in Taiwan. Health Economics, 9: 319-326.

MARGGRAF, R. (2005): Ökonomische Grundlagen der Umweltbewertung. In: Marggraf, R.; Bräuer, I; Fischer, A.; Menzel, S.; Stratmann, U. und Suhr, A.: Ökonomische Bewertung bei umweltrelevanten Entscheidungen. Einsatzmöglichkeiten von Zahlungsbereitschaftsanalysen in Politik und Verwaltung. Metropolis-Verlag, Marburg.

Mount, T., Weng, W.; Schulze, W. und Chestnut, L. (2001): Automobile Safety and the Value of Statistical Life in the Family: Valuing Reduced Risks for Children, Adults and the Elderly. Paper presented at the 2001 Association of Environmental and Resource Economists Workshop, Bar Harbor, Maine, June 13-15.

OECD (2006-2008): Valuation of Environment-Related Health Impacts with a particular Focus on Children (VERHI Children). http://www.oecd.org/site/0,3407,en_21571361_36146795_ 1_1_1_1_1,00.html zugegriffen am 28.09.2009.

RoycE, S. E. (1992): Case Studies in Environmental Medicine: Lead Toxicity, ATSDR. Department of Health and Human Services. 8, Atlanta.

SCAPECCHI, P. (2006): Valuation Differences between Adults and Children. In: Economic valuation of environmental health risks to children. OECD, Paris. $\mathrm{http}: / /$ masetto.sourceoecd.org/vl=5785535/cl=11/nw=1/rpsv/cgi-bin/fulltextew.pl?prpsv= /ij/oecdthemes/99980061/v2006n2/s1/p11.idx zugegriffen am: 10.06.2009

SCAPECCHI, P. (2007): Use of Evaluation Tools in Policymaking and Health Implications for Children. OECD, Paris. http://www.oecd.org/dataoecd/6/51/39647256.pdf zugegriffen am 19.06.2009.

SChnell, R.; Hill, P. B. und Esser, E. (1999): Methoden der empirischen Sozialforschung. R. Oldenbourg Verlag, München, Wien.

SCHÖFFSKI, O. (2007): Grundformen Gesundheitsökonomischer Evaluationen. In: Schöffski, O.; Schulenburg, J.-M. [Hrsg.], (2007): Gesundheitsökonomische Evaluationen, Springer, Berlin. SCHÖFFSKI a, O. (2007): Nutzentheoretische Lebensqualitätsmessung. In: Schöffski, O.; Schulenburg, J.-M. [Hrsg.] (2007): Gesundheitsökonomische Evaluationen. Springer, Berlin.

Slovic, P. (1987): Perceptions of Risk. Science, 30(4): 423-439. 
TAmburlini, G. (2006): Overview of the Risks Differences between Children and Adults. In: Economic Valuation of Environmental Health Risks to Children. OECD, Paris.

Tolley, G. und Fabian, R. (1999): Contingent Valuation and Valuing Children's Health. Paper prepared for U.S. EPA Environmental Policy and Economics Workshop Valuing Health for Environmental Policy with Special Emphasis on Children's Health Issues. March 24-25, 1999, Silver Spring, MD.

U.S. EnVIRonmental Protection Agency (U.S. EPA) (2009): Background document supporting the workgroup`s recommendations to CHAP on children's health valuation issues. http://yosemite.epa.gov/OCHP/OCHPWEB.nsf/content/whatwe_recomm_bckgrnd.htm zugegriffen am 19.06.2009.

U.S. Environmental Protection Agency (U.S. EPA) (2000): Guidelines for Preparing Economic Analyses. EPA 240-R-00-003. Washington, D.C.

U.S. Environmental Protection Agency (U.S. EPA), Office of Children's Health Protection; Office of Policy Economics and Innovation; National Center for ENVIRONMENTAL ECONOMICS (2003): Children’s Health Valuation Handbook. EPA 100-R03-003. Washington, D.C.

Übereinkommen über die Rechte des Kindes. UN-Kinderrechtskonvention im Wortlaut mit Materialien vom 20. November 1989. http://www.national-coalition.de/pdf/UNKinderrechtskonvention.pdf zugegriffen am 21.09.2009.

Umweltbundesamt: Aktionsprogramm Umwelt und Gesundheit: Berücksichtigung der Risikogruppe Kind bei der Ableitung gesundheitsbezogener Umweltstandards http://www.apug.de/kinder /projekte/kind-gesundheitsstandards.htm zugegriffen am 26.10.2009.

United Nations Treaty Collection http://treaties.un.org/Pages/ViewDetails.aspx src= TREATY\&mtdsg_no=IV-11\&chapter=4\&lang=en zugegriffen am 21.09.2009.

Viscusi, W. K.; MAgat, W. und HubeR, J. (1991): Communication of Ambiguous Risk Information. Theory and Decision, 31: 159-173.

Weltgesundheitsorganisation (WHO) (2003-2007): "Economic Valuation of transport-related health effects" (PEP).

Weltgesundheitsorganisation (WHO) (2008):,,Review methods and development of guidance for the economic valuation of transport-related health effects, with a particular focus on children“" (PEPa). http://www.euro.who.int/_data/assets/pdf_file/0008/53864/E92127.pdf zugegriffen am 28.09.2009. 
Working Party on National Environmental Policy (2005): The Valuation of Environmental Health Risks to Children: Methodological and Policy Issues. ENV/EPOC/WPNEP (2004) 15/FINAL, OECD. 


\section{Diskussionspapiere (2000 bis 2010): Institut für Agrarökonomie der Georg-}

\section{August-Universtät Göttingen; Department für Agrarökonomie und Rurale Entwicklung der Georg-August-Universtät Göttingen}

\begin{tabular}{|c|c|c|}
\hline 0001 & Brandes, Wilhelm & $\begin{array}{l}\text { Über Selbstorganisation in Planspielen: ein Erfahrungsbericht, } \\
2000\end{array}$ \\
\hline 0002 & $\begin{array}{l}\text { Von Cramon-Taubadel, } \\
\text { Stephan u. Jochen Meyer }\end{array}$ & Asymmetric Price Transmission: Factor Artefact?, 2000 \\
\hline 0101 & Leserer, Michael & Zur Stochastik sequentieller Entscheidungen, 2001 \\
\hline 0102 & Molua, Ernest & $\begin{array}{l}\text { The Economic Impacts of Global Climate Change on African } \\
\text { Agriculture, } 2001\end{array}$ \\
\hline 0103 & Birner, Regina et al. & $\begin{array}{l}\text {,Ich kaufe, also will ich?': eine interdisziplinäre Analyse der } \\
\text { Entscheidung für oder gegen den Kauf besonders tier- u. } \\
\text { umweltfreundlich erzeugter Lebensmittel, } 2001\end{array}$ \\
\hline \multirow[t]{2}{*}{0104} & Wilkens, Ingrid & $\begin{array}{l}\text { Wertschöpfung von Großschutzgebieten: Befragung von } \\
\text { Besuchern des Nationalparks Unteres Odertal als Baustein einer } \\
\text { Kosten-Nutzen-Analyse, } 2001\end{array}$ \\
\hline & & 2002 \\
\hline 0201 & Grethe, Harald & 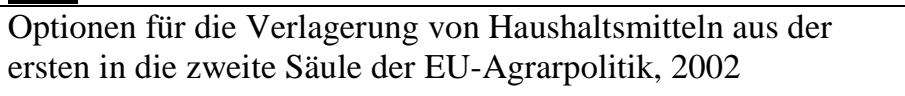 \\
\hline \multirow[t]{2}{*}{0202} & $\begin{array}{l}\text { Spiller, Achim u. Matthias } \\
\text { Schramm }\end{array}$ & $\begin{array}{l}\text { Farm Audit als Element des Midterm-Review : zugleich ein } \\
\text { Beitrag zur Ökonomie von Qualitätsicherungssytemen, } 2002\end{array}$ \\
\hline & & 2003 \\
\hline 0301 & Lüth, Maren et al. & 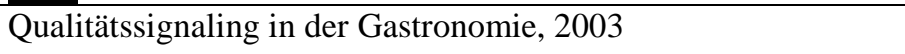 \\
\hline 0302 & $\begin{array}{l}\text { Jahn, Gabriele, Martina } \\
\text { Peupert u. Achim Spiller }\end{array}$ & $\begin{array}{l}\text { Einstellungen deutscher Landwirte zum QS-System: Ergebnisse } \\
\text { einer ersten Sondierungsstudie, } 2003\end{array}$ \\
\hline 0303 & Theuvsen, Ludwig & $\begin{array}{l}\text { Kooperationen in der Landwirtschaft: Formen, Wirkungen und } \\
\text { aktuelle Bedeutung, } 2003\end{array}$ \\
\hline \multirow[t]{2}{*}{0304} & Jahn, Gabriele & $\begin{array}{l}\text { Zur Glaubwürdigkeit von Zertifizierungssystemen: eine } \\
\text { ökonomische Analyse der Kontrollvalidität, } 2003\end{array}$ \\
\hline & & 2004 \\
\hline 0401 & $\begin{array}{l}\text { Meyer, Jochen u. S. von } \\
\text { Cramon-Taubadel }\end{array}$ & Asymmetric Price Transmission: a Survey, 2004 \\
\hline 0402 & $\begin{array}{l}\text { Barkmann, Jan u. Rainer } \\
\text { Marggraf }\end{array}$ & $\begin{array}{l}\text { The Long-Term Protection of Biological Diversity: Lessons } \\
\text { from Market Ethics, } 2004\end{array}$ \\
\hline 0403 & Bahrs, Enno & $\begin{array}{l}\text { VAT as an Impediment to Implementing Efficient Agricultural } \\
\text { Marketing Structures in Transition Countries, } 2004\end{array}$ \\
\hline 0404 & $\begin{array}{l}\text { Spiller, Achim, Torsten } \\
\text { Staack u. Anke Zühlsdorf }\end{array}$ & $\begin{array}{l}\text { Absatzwege für landwirtschaftliche Spezialitäten: Potenziale } \\
\text { des Mehrkanalvertriebs, } 2004\end{array}$ \\
\hline 0405 & $\begin{array}{l}\text { Spiller, Achim u. Torsten } \\
\text { Staack }\end{array}$ & $\begin{array}{l}\text { Brand Orientation in der deutschen Ernährungswirtschaft: } \\
\text { Ergebnisse einer explorativen Online-Befragung, } 2004\end{array}$ \\
\hline
\end{tabular}




\begin{tabular}{|c|c|c|}
\hline 0406 & $\begin{array}{l}\text { Gerlach, Sabine u. Berit } \\
\text { Köhler }\end{array}$ & $\begin{array}{l}\text { Supplier Relationship Management im Agribusiness: ein } \\
\text { Konzept zur Messung der Geschäftsbeziehungsqualität, } 2004\end{array}$ \\
\hline 0407 & Inderhees, Philipp et al. & Determinanten der Kundenzufriedenheit im Fleischerfachhandel \\
\hline \multirow[t]{2}{*}{0408} & Lüth, Maren et al. & $\begin{array}{l}\text { Köche als Kunden: Direktvermarktung landwirtschaftlicher } \\
\text { Spezialitäten an die Gastronomie, } 2004\end{array}$ \\
\hline & & 2005 \\
\hline 0501 & $\begin{array}{l}\text { Spiller, Achim, Julia } \\
\text { Engelken u. Sabine Gerlach }\end{array}$ & $\begin{array}{l}\text { Zur Zukunft des Bio-Fachhandels: eine Befragung von Bio- } \\
\text { Intensivkäufern, } 2005\end{array}$ \\
\hline 0502 & Groth, Markus & $\begin{array}{l}\text { Verpackungsabgaben und Verpackungslizenzen als Alternative } \\
\text { für ökologisch nachteilige Einweggetränkeverpackungen?: eine } \\
\text { umweltökonomische Diskussion, } 2005\end{array}$ \\
\hline 0503 & $\begin{array}{l}\text { Freese, Jan u. Henning } \\
\text { Steinmann }\end{array}$ & $\begin{array}{l}\text { Ergebnisse des Projektes 'Randstreifen als Strukturelemente in } \\
\text { der intensiv genutzten Agrarlandschaft Wolfenbüttels', } \\
\text { Nichtteilnehmerbefragung NAU 2003, } 2005\end{array}$ \\
\hline 0504 & $\begin{array}{l}\text { Jahn, Gabriele, Matthias } \\
\text { Schramm u. Achim Spiller }\end{array}$ & $\begin{array}{l}\text { Institutional Change in Quality Assurance: the Case of Organic } \\
\text { Farming in Germany, } 2005\end{array}$ \\
\hline \multirow[t]{2}{*}{0505} & $\begin{array}{l}\text { Gerlach, Sabine, Raphael } \\
\text { Kennerknecht u. Achim } \\
\text { Spiller }\end{array}$ & $\begin{array}{l}\text { Die Zukunft des Großhandels in der Bio-Wertschöpfungskette, } \\
2005\end{array}$ \\
\hline & & 2006 \\
\hline 0601 & $\begin{array}{l}\text { Heß, Sebastian, Holger } \\
\text { Bergmann u. Lüder } \\
\text { Sudmann }\end{array}$ & $\begin{array}{l}\text { Die Förderung alternativer Energien: eine kritische } \\
\text { Bestandsaufnahme, } 2006\end{array}$ \\
\hline 0602 & $\begin{array}{l}\text { Gerlach, Sabine u. Achim } \\
\text { Spiller }\end{array}$ & $\begin{array}{l}\text { Anwohnerkonflikte bei landwirtschaftlichen Stallbauten: } \\
\text { Hintergründe und Einflussfaktoren; Ergebnisse einer } \\
\text { empirischen Analyse, } 2006\end{array}$ \\
\hline 0603 & Glenk, Klaus & $\begin{array}{l}\text { Design and Application of Choice Experiment Surveys in So- } \\
\text { Called Developing Countries: Issues and Challenges, } 2006\end{array}$ \\
\hline 0604 & $\begin{array}{l}\text { Bolten, Jan, Raphael } \\
\text { Kennerknecht u. Achim } \\
\text { Spiller }\end{array}$ & $\begin{array}{l}\text { Erfolgsfaktoren im Naturkostfachhandel: Ergebnisse einer } \\
\text { empirischen Analyse, } 2006 \text { (entfällt) }\end{array}$ \\
\hline 0605 & Hasan, Yousra & $\begin{array}{l}\text { Einkaufsverhalten und Kundengruppen bei Direktvermarktern } \\
\text { in Deutschland: Ergebnisse einer empirischen Analyse, } 2006\end{array}$ \\
\hline 0606 & $\begin{array}{l}\text { Lülfs, Frederike u. Achim } \\
\text { Spiller }\end{array}$ & $\begin{array}{l}\text { Kunden(un-)zufriedenheit in der Schulverpflegung: Ergebnisse } \\
\text { einer vergleichenden Schulbefragung, } 2006\end{array}$ \\
\hline \multirow[t]{2}{*}{0607} & $\begin{array}{l}\text { Schulze, Holger, Friederike } \\
\text { Albersmeier u. Achim } \\
\text { Spiller }\end{array}$ & $\begin{array}{l}\text { Risikoorientierte Prüfung in Zertifizierungssystemen der Land- } \\
\text { und Ernährungswirtschaft, } 2006\end{array}$ \\
\hline & & 2007 \\
\hline 0701 & $\begin{array}{l}\text { Buchs, Ann Kathrin u. Jörg } \\
\text { Jasper }\end{array}$ & $\begin{array}{l}\text { For whose Benefit? Benefit-Sharing within Contractural ABC- } \\
\text { Agreements from an Economic Prespective: the Example of } \\
\text { Pharmaceutical Bioprospection, } 2007\end{array}$ \\
\hline 0702 & Böhm, Justus et al. & $\begin{array}{l}\text { Preis-Qualitäts-Relationen im Lebens- } \\
\text { Mittelmarkt: eine Analyse auf Basis der Testergebnisse Stiftung } \\
\text { Warentest, } 2007\end{array}$ \\
\hline
\end{tabular}




\begin{tabular}{|c|c|c|}
\hline 0703 & $\begin{array}{l}\text { Hurlin, Jörg u. Holger } \\
\text { Schulze }\end{array}$ & $\begin{array}{l}\text { Möglichkeiten und Grenzen der Qualitäts-sicherung in der } \\
\text { Wildfleischvermarktung, } 2007\end{array}$ \\
\hline & Ab Heft 4, 2007: & $\begin{array}{l}\text { Diskussionspapiere(Discussion Papers), Department für } \\
\text { Agrarökonomie und Rurale Entwicklung der Georg- } \\
\text { August-Universität, Göttingen (ISSN 1865-2697) }\end{array}$ \\
\hline 0704 & $\begin{array}{l}\text { Stockebrand, Nina u. Achim } \\
\text { Spiller }\end{array}$ & $\begin{array}{l}\text { Agrarstudium in Göttingen: Fakultätsimage und } \\
\text { Studienwahlentscheidungen; Erstsemesterbefragung im WS } \\
2006 / 2007\end{array}$ \\
\hline 0705 & $\begin{array}{l}\text { Bahrs, Enno, Jobst-Henrik } \\
\text { Held u. Jochen Thiering }\end{array}$ & $\begin{array}{l}\text { Auswirkungen der Bioenergieproduktion auf die Agrarpolitik } \\
\text { sowie auf Anreizstrukturen in der Landwirtschaft: eine partielle } \\
\text { Analyse bedeutender Fragestellungen anhand der Beispielregion } \\
\text { Niedersachsen }\end{array}$ \\
\hline \multirow[t]{2}{*}{0706} & $\begin{array}{l}\text { Yan, Jiong, } \\
\text { Jan Barkmann u. } \\
\text { Rainer Marggraf }\end{array}$ & $\begin{array}{l}\text { Chinese tourist preferences for nature based destinations - a } \\
\text { choice experiment analysis }\end{array}$ \\
\hline & & 2008 \\
\hline 0801 & $\begin{array}{l}\text { Joswig, Anette u. } \\
\text { Anke Zühlsdorf }\end{array}$ & $\begin{array}{l}\text { Marketing für Reformhäuser: } \\
\text { Senioren als Zielgruppe }\end{array}$ \\
\hline 0802 & $\begin{array}{l}\text { Schulze, Holger u. } \\
\text { Achim Spiller }\end{array}$ & $\begin{array}{l}\text { Qualitätssicherungssysteme in der europäischen Agri-Food } \\
\text { Chain: } \\
\text { Ein Rückblick auf das letzte Jahrzehnt }\end{array}$ \\
\hline 0803 & $\begin{array}{l}\text { Gille, Claudia u. } \\
\text { Achim Spiller }\end{array}$ & $\begin{array}{l}\text { Kundenzufriedenheit in der Pensionspferdehaltung: } \\
\text { eine empirische Studie }\end{array}$ \\
\hline 0804 & $\begin{array}{l}\text { Voss, Julian u. } \\
\text { Achim Spiller }\end{array}$ & $\begin{array}{l}\text { Die Wahl des richtigen Vertriebswegs in den } \\
\text { Vorleistungsindustrien der Landwirtschaft - } \\
\text { Konzeptionelle Überlegungen und empirische } \\
\text { Ergebnisse }\end{array}$ \\
\hline 0805 & $\begin{array}{l}\text { Gille, Claudia u. } \\
\text { Achim Spiller }\end{array}$ & $\begin{array}{l}\text { Agrarstudium in Göttingen. } \\
\text { Erstsemester- und Studienverlaufsbefragung } \\
\text { im WS 2007/08 }\end{array}$ \\
\hline 0806 & $\begin{array}{l}\text { Schulze, Birgit, } \\
\text { Christian Wocken u. } \\
\text { Achim Spiller }\end{array}$ & $\begin{array}{l}\text { (Dis)loyalty in the German dairy industry. } \\
\text { A supplier relationship management view } \\
\text { Empirical evidence and management implications }\end{array}$ \\
\hline 0807 & $\begin{array}{l}\text { Brümmer, Bernhard, } \\
\text { Ulrich Köster u. } \\
\text { Jens- Peter Loy }\end{array}$ & $\begin{array}{l}\text { Tendenzen auf dem Weltgetreidemarkt: } \\
\text { Anhaltender Boom oder kurzfristige } \\
\text { Spekulationsblase? }\end{array}$ \\
\hline 0808 & $\begin{array}{l}\text { Schlecht, Stehanie, } \\
\text { Friederike Albersmeier } \\
\text { u. Achim Spiller }\end{array}$ & $\begin{array}{l}\text { Konflikte bei landwirtschaftlichen Stallbauprojekten: } \\
\text { Eine empirische Untersuchung zum Bedrohungspotential } \\
\text { kritischer Stakeholder }\end{array}$ \\
\hline 0809 & $\begin{array}{l}\text { Lülfs-Baden,Frederike } \\
\text { u.Achim Spiller }\end{array}$ & $\begin{array}{l}\text { Steuerungsmechanismen im deutschen } \\
\text { Schulverpflegungsmarkt: eine institutionenökonomische } \\
\text { Analyse }\end{array}$ \\
\hline 0810 & $\begin{array}{l}\text { Deimel, Mark, } \\
\text { Ludwig Theuvsen u. } \\
\text { Christof Ebbeskotte }\end{array}$ & $\begin{array}{l}\text { Von der Wertschöpfungskette zum Netzwerk: } \\
\text { Methodische Ansätze zur Analyse des } \\
\text { Verbundsystems der Veredelungswirtschaft } \\
\text { Nordwestdeutschlands }\end{array}$ \\
\hline 0811 & $\begin{array}{l}\text { Albersmeier,Friederike } \\
\text { u. Achim Spiller }\end{array}$ & Supply Chain Reputation in der Fleischwirtschaft \\
\hline
\end{tabular}




\begin{tabular}{|c|c|c|}
\hline & & 2009 \\
\hline 0901 & $\begin{array}{l}\text { Bahlmann, Jan, } \\
\text { Achim Spiller u. } \\
\text { Cord-Herwig Plumeyer }\end{array}$ & $\begin{array}{l}\text { Status quo und Akzeptanz von Internet-basierten } \\
\text { Informationssystemen: } \\
\text { Ergebnisse einer empirischen Analyse in der } \\
\text { deutschen Veredelungswirtschaft }\end{array}$ \\
\hline 0902 & $\begin{array}{l}\text { Gille, Claudia } \\
\text { u. Achim Spiller }\end{array}$ & $\begin{array}{l}\text { Agrarstudium in Göttingen. } \\
\text { Eine vergleichende Untersuchung der Erstsemester } \\
\text { der Jahre 2006-2009 }\end{array}$ \\
\hline 0903 & $\begin{array}{l}\text { Gawron, Jana-Christina u. } \\
\text { Ludwig Theuvsen }\end{array}$ & $\begin{array}{l}\text { „Zertifizierungssysteme des Agribusiness im interkulturellen } \\
\text { Kontext - Forschungsstand und Darstellung der kulturellen } \\
\text { Unterschiede” }\end{array}$ \\
\hline 0904 & $\begin{array}{l}\text { Raupach, Katharina u. } \\
\text { Rainer Marggraf }\end{array}$ & $\begin{array}{l}\text { Verbraucherschutz vor dem Schimmelpilzgift Deoxynivalenol } \\
\text { in Getreideprodukten } \\
\text { Aktuelle Situation und Verbesserungsmöglichkeiten }\end{array}$ \\
\hline 0905 & $\begin{array}{l}\text { Busch,Anika u. } \\
\text { Rainer Marggraf }\end{array}$ & $\begin{array}{l}\text { Analyse der deutschen globalen Waldpolitik im Kontext der } \\
\text { Klimarahmenkonvention und des Übereinkommens über die } \\
\text { Biologische Vielfalt }\end{array}$ \\
\hline 0906 & $\begin{array}{l}\text { Zschache, Ulrike, } \\
\text { Stephan v.Cramon- } \\
\text { Taubadel und Ludwig } \\
\text { Theuvsen }\end{array}$ & $\begin{array}{l}\text { Die öffentliche Auseinandersetzung über Bioenergie in den } \\
\text { Massenmedien } \\
\text { Diskursanalytische Grundlagen und erste Ergebnisse }\end{array}$ \\
\hline 0907 & $\begin{array}{l}\text { Onumah, Edward E., } \\
\text { Gabriele Hoerstgen- } \\
\text { Schwark and Bernhard } \\
\text { Brümmer }\end{array}$ & $\begin{array}{l}\text { Productivity of hired and family labour and determinants of } \\
\text { technical inefficiency in Ghana's fish farms }\end{array}$ \\
\hline 0908 & $\begin{array}{l}\text { Onumah, Edward E., } \\
\text { Stephan Wessels, Nina } \\
\text { Wildenhayn, Gabriele } \\
\text { Hoerstgen-Schwark and } \\
\text { Bernhard Brümmer }\end{array}$ & $\begin{array}{l}\text { Effects of stocking density and photoperiod manipulation in } \\
\text { relation to estradiol profile to enhance } \\
\text { spawning activity in female Nile tilapia }\end{array}$ \\
\hline 0909 & $\begin{array}{l}\text { Steffen, Nina, Stephanie } \\
\text { Schlecht u. Achim Spiller }\end{array}$ & Ausgestaltung von Milchlieferverträgen nach der Quote \\
\hline 0910 & $\begin{array}{l}\text { Steffen, Nina, Stephanie } \\
\text { Schlecht u. Achim Spiller }\end{array}$ & Das Preisfindungssystem von Genossenschaftsmolkereien \\
\hline 0911 & $\begin{array}{l}\text { Granoszewski, Karol, } \\
\text { Christian Reise, } \\
\text { Achim Spiller und } \\
\text { Oliver Mußhoff }\end{array}$ & $\begin{array}{l}\text { Entscheidungsverhalten landwirtschaftlicher Betriebsleiter bei } \\
\text { Bioenergie-Investitionen } \\
\text { - Erste Ergebnisse einer empirischen Untersuchung - }\end{array}$ \\
\hline 0912 & $\begin{array}{l}\text { Albersmeier, F., } \\
\text { Mörlein, D. und } \\
\text { Achim Spiller }\end{array}$ & $\begin{array}{l}\text { Zur Wahrnehmung der Qualität von Schweinefleisch beim } \\
\text { Kunden. }\end{array}$ \\
\hline 0913 & $\begin{array}{l}\text { Ihle, R., } \\
\text { Brümmer, B. und } \\
\text { Stanley R. Thompson }\end{array}$ & $\begin{array}{l}\text { Spatial Market Integration in the EU Beef and Veal Sector: } \\
\text { Policy Decoupling and Export Bans }\end{array}$ \\
\hline
\end{tabular}




\begin{tabular}{|c|l|l|}
\hline $\mathbf{1 0 0 1}$ & $\begin{array}{l}\text { Sebastian Hess, } \\
\text { Stephan v. Cramon- } \\
\text { Taubadel und Stefan } \\
\text { Sperlich }\end{array}$ & $\begin{array}{l}\text { Numbers for Pascal: Explaining differences in the estimated } \\
\text { benefits of the Doha Development Agenda }\end{array}$ \\
\hline $\mathbf{1 0 0 2}$ & $\begin{array}{l}\text { Deimel, I., J. Böhm u. B. } \\
\text { Schulze }\end{array}$ & $\begin{array}{l}\text { Low Meat Consumption als Vorstufe zum Vegetarismus? Eine } \\
\text { qualitative Studie zu den Motivstrukturen geringen } \\
\text { Fleischkonsums }\end{array}$ \\
\hline $\mathbf{1 0 0 3}$ & Franz, A. u. B. Nowak & $\begin{array}{l}\text { Functional food consumption in Germany: A lifestyle } \\
\text { segmentation study }\end{array}$ \\
\hline $\mathbf{1 0 0 4}$ & Deimel, M. u. L. Theuvsen & $\begin{array}{l}\text { Standortvorteil Nordwestdeutschland? Eine Untersuchung zum } \\
\text { Einfluss von Netzwerk- und Clusterstrukturen in der } \\
\text { Schweinefleischerzeugung }\end{array}$ \\
\hline
\end{tabular}




\title{
Diskussionspapiere (2000 bis 31. Mai 2006): Institut für Rurale Entwicklung der Georg-August-Universität, Göttingen
}

\author{
Ed. Winfried Manig (ISSN 1433-2868)
}

\begin{tabular}{|c|c|c|}
\hline 32 & Dirks, Jörg J. & $\begin{array}{l}\text { Einflüsse auf die Beschäftigung in } \\
\text { nahrungsmittelverabeitenden ländlichen Kleinindustrien in } \\
\text { West-Java/Indonesien, } 2000\end{array}$ \\
\hline 33 & Keil, Alwin & Adoption of Leguminous Tree Fallows in Zambia, 2001 \\
\hline 34 & Schott, Johanna & $\begin{array}{l}\text { Women's Savings and Credit Co-operatives in Madagascar, } \\
2001\end{array}$ \\
\hline 35 & Seeberg-Elberfeldt, Christina & $\begin{array}{l}\text { Production Systems and Livelihood Strategies in Southern } \\
\text { Bolivia, } 2002\end{array}$ \\
\hline 36 & Molua, Ernest L. & $\begin{array}{l}\text { Rural Development and Agricultural Progress: Challenges, } \\
\text { Strategies and the Cameroonian Experience, } 2002\end{array}$ \\
\hline 37 & Demeke, Abera Birhanu & $\begin{array}{l}\text { Factors Influencing the Adoption of Soil Conservation } \\
\text { Practices in Northwestern Ethiopia, } 2003\end{array}$ \\
\hline 38 & $\begin{array}{l}\text { Zeller, Manfred u. Julia } \\
\text { Johannsen }\end{array}$ & $\begin{array}{l}\text { Entwicklungshemmnisse im afrikanischen Agrarsektor: } \\
\text { Erklärungsansätze und empirische Ergebnisse, } 2004\end{array}$ \\
\hline 39 & Yustika, Ahmad Erani & $\begin{array}{l}\text { Institutional Arrangements of Sugar Cane Farmers in East Java } \\
\text { - Indonesia: Preliminary Results, } 2004\end{array}$ \\
\hline 40 & Manig, Winfried & $\begin{array}{l}\text { Lehre und Forschung in der Sozialökonomie der Ruralen } \\
\text { Entwicklung, } 2004\end{array}$ \\
\hline 41 & Hebel, Jutta & $\begin{array}{l}\text { Transformation des chinesischen Arbeitsmarktes: } \\
\text { gesellschaftliche Herausforderungen des } \\
\text { Beschäftigungswandels, } 2004\end{array}$ \\
\hline 42 & Khan, Mohammad Asif & $\begin{array}{l}\text { Patterns of Rural Non-Farm Activities and Household Acdess } \\
\text { to Informal Economy in Northwest Pakistan, } 2005\end{array}$ \\
\hline 43 & Yustika, Ahmad Erani & $\begin{array}{l}\text { Transaction Costs and Corporate Governance of Sugar Mills in } \\
\text { East Java, Indovesia, } 2005\end{array}$ \\
\hline 44 & $\begin{array}{l}\text { Feulefack, Joseph Florent, } \\
\text { Manfred Zeller u. Stefan } \\
\text { Schwarze }\end{array}$ & $\begin{array}{l}\text { Accuracy Analysis of Participatory Wealth Ranking (PWR) in } \\
\text { Socio-economic Poverty Comparisons, } 2006\end{array}$ \\
\hline
\end{tabular}




\subsection{Mykotoxine als Gesundheitsrisiko für Kinder - Regulierungspräferenzen aus}

\section{Elternsicht}

Niens, C. und Marggraf, R. (2013): Mykotoxine als Gesundheitsrisiko für Kinder Regulierungspräferenzen aus Elternsicht. Short Paper. Tagungsband der 23. Jahrestagung der Österreichischen Gesellschaft für Agrarökonomie gemeinsam mit der 41. Jahrestagung der Schweizer Gesellschaft für Agrarwirtschaft und Agrarsoziologie. Verfügbar unter: http://oega.boku.ac.at/fileadmin/user upload/Tagung/2013/Short Papers 2013/SGAOEGA-TAGUNGSBAND 2013.pdf 


\title{
Mykotoxine als Gesundheitsrisiko für Kinder Regulierungspräferenzen aus Elternsicht
}

\author{
Christine Niens und Rainer Marggraf ${ }^{1}$
}

\begin{abstract}
Die Aufnahme von Mykotoxinen stellt für den Verbraucher ein Gesundheitsrisiko dar. Besonders Kinder können auch bei Einhaltung der gesetzlichen Grenzwerte $z u$ viele Mykotoxine aufnehmen und dadurch in ihrer Gesundheit geschädigt werden. Vor diesem Hintergrund wurden mithilfe eines Choice Experiments die elterlichen Regulierungspräferenzen für verschiedene Verbesserungsoptionen des Mykotoxin-Risikomanagements ermittelt. Es zeigt sich, dass Eltern eine Verbesserung des Gesundheitsschutzes von Kindern wünschen, wobei eine Senkung der Mykotoxingrenzwerte der Einführung spezieller „Kindergetreideprodukte" vorgezogen wird.
\end{abstract}

\section{EINLEITUNG}

Mykotoxine sind natürlich vorkommende Schimmelpilzgifte, die die Sicherheit getreidehaltiger Lebensmittel beeinträchtigen können. Sie werden u. a. von Pilzen der Gattung Fusarium gebildet, wobei Deoxynivalenol (DON) das häufigste Fusarium-Mykotoxin im Getreide ist (Bartels und Rodemann, 2003). Besonders in Weizen wird DON regelmäßig in bedeutsamen Konzentrationen nachgewiesen.

Die Aufnahme von Mykotoxinen stellt für den Verbraucher ein Gesundheitsrisiko dar. So konnte für DON u. a. eine immunsuppressive Wirkung belegt werden (SCF, 1999). Um die öffentliche Gesundheit vor Beeinträchtigungen zu schützen, wurden Grenzwerte für die maximale Belastung von Lebensmitteln mit Mykotoxinen erlassen (VO (EG) Nr. 1881/2006).

Verschiedene Studien zeigen jedoch, dass auch bei Einhaltung der gesetzlichen Höchstgehalte die Möglichkeit besteht, dass kleine Kinder mehr als eine unbedenkliche Menge DON aufnehmen (Raupach, 2012; Curtui et al., 2006) und folglich in ihrer Gesundheit geschädigt werden können.

\section{RISIKOREGULIERUNGS-OPTIONEN}

Eine Verbesserung des gesundheitlichen Verbraucherschutzes von Kindern vor DON könnte grundsätzlich durch zwei Maßnahmen erreicht werden. Entweder durch a) eine generelle Senkung der Mykotoxingrenzwerte für alle Getreideprodukte in Deutschland, oder b) die Bereitstellung besonders sicherer "Kindergetreideprodukte", die nur so viele Mykotoxine enthalten, dass ein Gesundheitsrisiko für Kinder ausgeschlossen werden kann. Da die Vermeidung von Mykotoxinen aber mit Mehrkosten für die Lebensmittelunternehmer verbunden ist (Niens und

${ }^{1} \mathrm{C}$. Niens ist Wissenschaftliche Mitarbeiterin, R. Marggraf ist Leiter der Abteilung Umwelt- und Ressourcenökonomik des Departments für Agrarökonomie und Rurale Entwicklung der Universität Göttingen.
Hasselmann, 2011), führen beide Regulierungsoptionen zu Preiserhöhungen für getreidehaltige Lebensmittel. Fraglich ist, ob Eltern eine Verbesserung des Gesundheitsschutzes von Kindern vor DON wünschen und welche Maßnahme sie präferieren.

\section{METHODIK}

Im Januar 2012 wurden insgesamt 771 Fragebögen an Eltern aus Niedersachsen (Deutschland) mit Kindern im Alter zwischen drei und elf Jahren versandt. Die Befragungsteilnehmer wurden schriftlich darauf hingewiesen, dass der Bogen von der Person ausgefüllt werden sollte, die hauptverantwortlich für den Lebensmitteleinkauf der Familie ist. Um sicherzustellen, dass die Befragten informierte Entscheidungen treffen können, enthielt der Fragebogen einen Informationstext bezüglich des Vorkommens von Mykotoxinen, ihrem Potential zur Gesundheitsschädigung und der derzeitigen gesetzlichen Regulierung.

Die Regulierungspräferenzen der Eltern bezüglich der genannten Optionen a) "Grenzwertsenkung" und b) "Kindergetreideprodukte" wurden mithilfe eines Discrete Choice Experiments (DCE) erhoben. Hierzu wurde mittels der Software Ngene ein orthogonales Design konstruiert, welches sich zudem minimal hinsichtlich der Attributausprägungen, über die Alternativen eines Choice Sets hinweg, überschnitt (Burgess und Street, 2005). Es resultierten acht Choice Sets mit je drei Alternativen, wovon die Dritte jeweils dem Status Quo (keine neuen Maßnahmen zur Verbesserung des Gesundheitsschutzes; Lebensmittelausgaben bleiben unverändert) entsprach. Die Attribute des DCE und ihre möglichen Ausprägungen werden in Tabelle 1 dargestellt.

Tabelle 1. Attribute des DCE und mögliche Ausprägungen

\begin{tabular}{ll}
\hline Attribut & Ausprägung \\
\hline Regulierungsoption & $\begin{array}{l}\text { Senkung Mykotoxin- } \\
\text { grenzwerte, Bereitstellung } \\
\end{array}$ \\
& "Kindergetreideprodukte" \\
Preis (Anstieg Lebensmittel- & $5,10,15,20$
\end{tabular}
ausgaben pro Monat in \%)

Allen Befragten wurden acht Choice Sets zur Bewertung vorgelegt (full factorial). Des Weiteren wurden soziodemographische Merkmale von Eltern und Kindern erfasst. Die Auswertung des DCE erfolgte mittels StataIC10 unter Anwendung von Mixed Logit Modellen (ML), um dem Panelcharakter der Daten zu entsprechen. Es wurden sowohl ein Einzelmodell, das nur die Attribute des DCE enthielt, als auch 
Gesamtmodelle, in denen alle soziodemographischen Merkmale gleichzeitig berücksichtigt werden konnten, berechnet. Dabei wurden die Regulierungsoptionen als Zufallsparameter, unter Normalverteilungsannahme, definiert. Als Referenz diente in allen Modellen der Status Quo. Präferenzunterschiede hinsichtlich der Verbesserungsoptionen wurden aus dem Einzelmodell nach Hole (2007) berechnet: $100 * \Phi\left(-b_{k} / s d_{k}\right)$, wobei $\Phi$ der Standardnormalverteilung entspricht, $b_{k}$ dem Mittelwert und $s d_{k}$ der Standardabweichung des $k$-ten Koeffizienten im ML.

\section{ERGEBNISSE}

Insgesamt wurden 238 Fragebögen zurückgesandt, was einer Rücklaufquote von 30,9\% entspricht. Die Kinder, auf die sich die Befragten bei der Beantwortung beziehen, sind zu 49,2\% Mädchen und zu $50,8 \%$ Jungen. Der überwiegende Teil der Kinder $(76 \%)$ ist zwischen 2005 und 2008 geboren. Mehr als die Hälfte aller befragten Eltern $(52,3 \%)$ besitzen ein Abitur oder einen höheren Bildungsabschluss.

Die Ergebnisse des DCE sind in Tabelle 2 zusammengefasst. Aus den Schätzungen des MLs (Gesamtmodell) geht hervor, dass alle Attribute des DCE einen signifikanten Einfluss auf die Auswahlentscheidung der Befragten haben. Das negative Vorzeichen des Preisattributes deutet darauf hin, dass ein Anstieg der monatlichen Lebensmittelausgaben von den befragten Eltern im Durchschnitt negativ bewertet wird. Das positive Vorzeichen der Attribute "Grenzwertsenkung" und "Kindergetreideprodukte" lässt darauf schließen, dass beide Verbesserungsoptionen gegenüber dem Status Quo präferiert werden. Dabei wird die Senkung der Mykotoxingrenzwerte im Durchschnitt positiver bewertet als die speziellen „Kindergetreideprodukte" (Tabelle 2).

Tabelle 2. Elterliche Präferenzen bezüglich der Verbesserung des Gesundheitsschutzes von Kindern vor Mykotoxinen und Einfluss soziodemographischer Variablen (ML).

\begin{tabular}{|c|c|c|}
\hline Attribut/Interaktion & $\begin{array}{c}\text { Koeffizienten } \\
\text { (Mittelwert) }\end{array}$ & $\begin{array}{c}\text { Standard- } \\
\text { abweichung }\end{array}$ \\
\hline Preis & $-.2115 * * * *$ & \\
\hline Grenzwertsenkung (GW) & $5.9480 * * * *$ & $3.4091 * * * *$ \\
\hline Kindergetreideprodukte (KP) & $3.6160 * * * *$ & $2.6567 * * * *$ \\
\hline Einkommen*GW & .0002 & \\
\hline Einkommen*KP & .0002 & \\
\hline Bildung*GW & -.0135 & \\
\hline Bildung*KP & -.0684 & \\
\hline Alter Befragter*GW & -.0195 & \\
\hline Alter Befragter*KP & .0161 & \\
\hline Haushaltsmitglieder*GW & $-.3980 * *$ & \\
\hline Haushaltsmitglieder*KP & $-.4919 * *$ & \\
\hline Alter Kind*GW & -.0741 & \\
\hline Alter Kind*KP & -.0457 & \\
\hline Geschlecht Kind*GW & -.0934 & \\
\hline Geschlecht Kind*KP & -.0799 & \\
\hline
\end{tabular}

Die signifikante Standardabweichung der Attribute "Grenzwertsenkung" und "Kindergetreideprodukte" zeigt, dass die Verbesserungsoptionen nicht von allen Eltern gleich bewertet werden. Die "Grenzwertsenkung" wird von 95\% der Befragten dem Status Quo vorgezogen. Die Bereitstellung besonders siche- rer "Kindergetreideprodukte" präferieren $87 \%$ der Eltern gegenüber dem derzeitigen MykotoxinRisikomanagement. Nur die Anzahl der Haushaltsmitglieder hat einen signifikanten Einfluss auf die Regulierungspräferenzen. Mit steigender Anzahl verringert sich die Wahrscheinlichkeit, dass Eltern eine der beiden Verbesserungsoptionen wählen. Weitere signifikante Interaktionen sind nicht nachweisbar. Die gefundenen Präferenzunterschiede können demnach nicht mit Persönlichkeitseigenschaften von Eltern und Kindern erklärt werden.

\section{ABSCHLIEBENDE BEMERKUNG}

Insgesamt sprechen sich die Befragten deutlich für eine Verbesserung des Gesundheitsschutzes von Kindern vor Mykotoxinen aus. Dabei wird die Senkung der Mykotoxingrenzwerte gegenüber den sicheren "Kindergetreideprodukten" präferiert. Aus praktischer Sicht erscheint eine Herabsetzung der Mykotoxingrenzwerte jedoch kaum umsetzbar. Entsprechend der VO (EG) Nr. 1881/2006 müssen Grenzwerte durch gute Landwirtschaftspraxis erreichbar sein. Es ist jedoch fraglich, ob auch in sog. „Fusariumjahren" ausreichend Getreide für die menschliche Ernährung produziert werden kann, das den strengeren Grenzwerten entspricht. Daher erscheint die Einführung von "Kindergetreideprodukten" eher realisierbar. Diese Studie hat gezeigt, dass auch "Kindergetreideprodukte" aus Elternsicht eine akzeptable Maßnahme darstellen, um den Gesundheitsschutz von Kindern vor Mykotoxinen zu verbessern.

\section{LITERATUR}

Amtsblatt der Europäischen Union L 346/5, Verordnung (EG) Nr. 1881/2006 vom 19. Dezember 2006 zur Festsetzung der Höchstgehalte für bestimmte Kontaminationen in Lebensmitteln.

Bartels, G. und Rodemann, B. (2003). Strategien zur Vermeidung von Mykotoxinen in Getreide. Gesunde Pflanzen 55(5):125-135.

Burgess, L. und Street D. J. (2005). Optimal designs for choice experiments with asymmetric attributes. Journal of Statistical Planning and Inferences 134: 288-301.

Curtui, V., Brockmaeyer, A., Dietrich, R., Kappenstein, O. Klaffke, H., Lepschy, J., Märtlbauer, E., Schneider, E., Seidler, C., Thielert, G., Usleber, E., Weber, R. und Wolff, J. (2006). Analytik und Vorkommen wichtiger Fusarientoxine. Münster: Landwirtschaftsverlag $\mathrm{GmbH}$.

Hole, A. R. (2007). Fitting mixed logit models by using maximum simulated likelihood. The Stata Journal 7(3): 388-401.

Niens, C. und Hasselmann, H. (2011). Ist eine Verbesserung des Verbraucherschutzes vor Mykotoxinen ökonomisch rational? Jahrbuch der Österreichischen Gesellschaft für Agrarökonomie 20(1):181-190.

Raupach, K. (2012). Risiko und Risikomanagement ausgewählter Fusarium-Mykotoxine. Ibidem-Verlag, Stuttgart.

Scientific Commitee on Food (SCF) (1999). Opinion on Fusarium Toxins Part 1: Deoxynivalenol (DON). Brüssel. 


\subsection{Mykotoxine und Kindergesundheit - Risikowahrnehmung und Zahlungsbereitschaft} für eine Risikoreduzierung aus Elternsicht

Niens, C. und Marggraf, R. (2013): Mykotoxine und Kindergesundheit - Risikowahrnehmung und Zahlungsbereitschaft für eine Risikoreduzierung aus Elternsicht. Jahrbuch der Österreichischen Gesellschaft für Agrarökonomie - Band 22 (1): 71-80. Facultas Verlag, Wien. Verfügbar unter: http://oega.boku.ac.at/fileadmin/user upload/Tagung/2012/Band 22 1/ 06 Niens Marggraf OEGA Jahrbuch2012.pdf 


\section{Mykotoxine und Kindergesundheit - Risikowahrnehmung und Zahlungsbereitschaft für eine Risikoreduzierung aus Elternsicht}

Mycotoxins and child health - Parental risk perception and willingness to pay for risk reduction

Christine NIENS und Rainer MARGGRAF

\section{Zusammenfassung}

Die Aufnahme von Mykotoxinen über die Nahrung stellt für den Verbraucher ein Gesundheitsrisiko dar. Besonders Kinder können, auch bei Einhaltung der gesetzlichen Vorgaben, zu viele Mykotoxine (Deoxynivalenol) aufnehmen und dadurch in ihrer Gesundheit geschädigt werden. Ausgehend davon werden die elterliche MykotoxinRisikowahrnehmung und die Zahlungsbereitschaft für eine Risikoreduzierung empirisch bestimmt. Mehr als die Hälfte der befragten Eltern geht davon aus, dass Mykotoxine eine nennenswerte Gefahr für die Gesundheit ihrer Kinder darstellen. Entsprechend besteht eine Mehrzahlungsbereitschaft für kindergerechte Getreideprodukte, die einen sicheren Schutz vor Mykotoxinen garantieren von durchschnittlich 43,58\% im Vergleich zum Preis herkömmlicher Produkte.

Schlagworte: Mykotoxine, Verbraucherschutz, Kindergesundheit, Elternbefragung

\section{Summary}

The ingestion of mycotoxins from cereal products poses a risk to consumers' health. Hence, cereal products underlie legal regulations concerning the maximum mycotoxin (deoxynivalenol) concentration. However, legislated maximum levels do not prevent children from over-stepping acceptable total daily intake. Against this background, a

Erschienen im Jahrbuch der ÖGA, Band xx, Jahr xxxx. Diese Fußnote bitte nicht löschen! Fußnotentext [AGRAR Fußnotentext]. 
parent survey was conducted to analyse the parental risk perception of mycotoxins related to child health and to assess the parental willingness to pay for risk reduction. More than half of the respondents believe that mycotoxins pose a serious health risk to their children. Accordingly, respondents are willing to pay a price premium of on average $43.58 \%$ for cereal products which contain only such an amount of mycotoxins that the health risk for children can be excluded.

Keywords: Mycotoxins, consumer protection, child health, parent survey

\section{Einleitung}

Mykotoxine sind natürlich vorkommende Schimmelpilzgifte, die die Sicherheit getreidehaltiger Lebensmittel beeinträchtigen können. Sie werden von Pilzen der Gattung Fusarium gebildet, wobei Deoxynivalenol (DON) das häufigste Fusarium-Mykotoxin im Getreide darstellt (BARTELS und RODEMANN, 2003). In Deutschland wird DON besonders in Weizen regelmäßig nachgewiesen (BMELV, 2010).

Die Aufnahme von Mykotoxinen stellt für den Verbraucher ein Gesundheitsrisiko dar. So konnte für DON u. a. eine immunsuppressive Wirkung belegt werden (SCF, 1999). Um die öffentliche Gesundheit vor Beeinträchtigungen $\mathrm{zu}$ schützen, wurden auf europäischer Ebene Grenzwerte für die maximale Belastung von Getreide und getreidehaltigen Lebensmitteln mit Mykotoxinen erlassen (VO (EG) Nr. 1881/ 2006).

Verschiedene Studien zeigen jedoch, dass auch bei Einhaltung der gesetzlichen Höchstgehalte die Möglichkeit besteht, dass Kinder mehr als die gesundheitlich unbedenkliche Menge DON aufnehmen und infolgedessen in ihrer Gesundheit geschädigt werden können (CURTUI et al., 2006, 111ff; RAUPACH, 2012, 115ff).

\section{Hintergrund und Zielsetzung}

In der Vergangenheit wurden unterschiedliche empirische Studien zur Laien- und Expertensicht auf die Mykotoxinproblematik durchgeführt (FREESE, 2010; RAUPACH und MARGGRAF, 2011). Diese Studien waren jedoch auf potentielle Gesundheitsrisiken durch Mykotoxine für Erwachsene bezogen. Obwohl Kinder eine Risikogruppe unter den Ver- 
braucherInnen darstellen und das Thema Mykotoxine für Eltern daher besondere Relevanz besitzt, wurde die elterliche Sicht auf die Mykotoxinproblematik bisher nicht erhoben. Kenntnisse über die elterliche Risikowahrnehmung werden jedoch benötigt, um angemessene Risikokommunikations- und Risikomanagementstrategien entwickeln zu können.

Vor diesem Hintergrund wird mithilfe eines Fragenbogens zunächst die elterliche Risikowahrnehmung von Mykotoxinen bestimmt. AuBerdem werden die Sicht auf die aktuelle gesetzliche Regulierung und die elterliche Wertschätzung für eine Reduzierung des Gesundheitsrisikos ihrer Kinder durch Mykotoxine ermittelt. Für die Erfassung dieser Wertschätzung wird das ökonomische Wertekonzept zugrunde gelegt. Entsprechend wird die elterliche Wertschätzung als maximale Zahlungsbereitschaft der Eltern für eine Reduzierung des Gesundheitsrisikos ihrer Kinder durch Mykotoxine definiert.

\section{Methodik}

Im Januar 2012 wurden Eltern aus Niedersachsen (Deutschland) mit Kindern zwischen vier und elf Jahren zu ihrer Risikowahrnehmung von Mykotoxinen und zur Zahlungsbereitschaft für eine Reduzierung des Gesundheitsrisikos ihrer Kinder schriftlich befragt. Hierfür wurden insgesamt 771 Fragebögen an 17 Kindertagestätten in Göttingen und Hannover versandt. Die Städte Göttingen und Hannover wurden als Befragungsregion ausgewählt, da sie hinsichtlich des Pro-KopfEinkommens repräsentativ für Niedersachsen sind. Die Fragebögen wurden von den ErzieherInnen der teilnehmenden Kindertagestätten an Eltern mit Kindern zwischen vier und elf Jahren weitergeben. Es wurde jeweils ein Fragebogen pro Haushalt ausgehändigt. Die BefragungsteilnehmerInnen wurden schriftlich darauf hingewiesen, dass der Bogen von der Person ausgefüllt werden sollte, die hauptverantwortlich für den Lebensmitteleinkauf der Familie ist. Außerdem wurden Befragte mit mehreren Kindern gebeten, bei der Beantwortung aller Fragen, die sich auf „ihr Kind“ beziehen, immer an das gleiche, zwischen 2001 und 2008 geborene Kind zu denken. Um sicherzustellen, dass die Befragten in der Lage sind, informierte Entscheidungen zu treffen, enthielt der Fragebogen objektive Informationen über das Vorkommen von Mykotoxinen, ihr Potential zur Gesundheitsschädigung 
und die gesetzliche Regulierung des Mykotoxinrisikos durch die Festlegung von Grenzwerten für Getreide und getreidehaltige Nahrungsmittel. Der Fragebogen wurde mittels kognitiver Pretests $(n=3)$ und eines Standardbeobachtungs-Pretests $(n=24)$ im Vorfeld der Studie getestet.

Im Rahmen der Befragung wurden folgende Konstrukte erfasst:

- Gesundheitsrisiko durch Mykotoxine für das eigene Kind und für Erwachsene: „Wie hoch schätzen Sie das Gesundheitsrisiko durch Mykotoxine in Getreideprodukten für Ihr Kind [... für Erwachsene] ein?" (1 = gar kein Risiko bis 7 = sehr hohes Risiko);

- Schädigungspotential von Mykotoxinen: „Was meinen Sie, wie ernsthaft können Mykotoxine die Gesundheit Ihres Kindes schädigen?"(1 = gar nicht ernsthaft bis 7 = sehr ernsthaft);

- Beurteilung des derzeitigen Mykotoxin-Risikomanagements: „Inwieweit halten Sie die aktuellen gesetzlichen Regelungen (Grenzwerte) für ausreichend, um Kinder vor Mykotoxinen $\mathrm{zu}$ schützen?"( 1 = gar nicht ausreichend bis 7 = vollkommen ausreichend) „Inwieweit liegt es in Ihrer Verantwortung oder in der Verantwortung des Staates, Ihr Kind vor einer Gesundheitsgefährdung durch Mykotoxine in Getreideprodukten zu schützen?" (1 = komplett in meiner Verantwortung bis $7=$ komplett in staatlicher Verantwortung)

Die Ermittlung der elterlichen Zahlungsbereitschaft für die Reduzierung des Mykotoxinrisikos erfolgte exemplarisch über die Mehrzahlungsbereitschaft für ein spezielles „Kinderweizenbrötchen“, das nur so viele Mykotoxine enthält, dass ein Gesundheitsrisiko für Kinder sicher ausgeschlossen werden kann. Hierfür wurde zunächst die elterliche Zahlungsbereitschaft für ein herkömmliches Weizenbrötchen mittels einer offenen Frage erhoben. Zur Bestimmung der Mehrzahlungsbereitschaft für ein besonders sicheres „Kinderweizenbrötchen“ wurde den Befragten eine siebenstufige Skala mit Beträgen von 0 Cent bis 30 Cent vorgelegt. Die Entscheidung, einen Betrag von 30 Cent als Skalenendpunkt zu benennen, beruht auf den Ergebnissen der Pretests. Abschließend wurden soziodemographische Merkmale von Eltern und Kindern sowie das Haushaltsnettoeinkommen erfasst. Das monatliche Haushaltsnettoeinkommen wurde mittels einer offenen Fragestellung und alternativ in Kategorien von unter $€ 1.000$ bis über $€ 7.000$ erfragt. Die Datenauswertung erfolgte mithilfe von SPSS 20. 


\section{Ergebnisse}

Insgesamt wurden 238 Fragebögen zurückgesandt, was einer Rücklaufquote von 30,9\% entspricht. Die Kinder, auf die sich die Befragten bei der Beantwortung beziehen, sind zu 49,2\% Mädchen und zu 50,8\% Jungen. Der überwiegende Teil der Kinder (76\%) ist zwischen 2005 und 2008 geboren (Mittelwert $(M)=6$ Jahre). Gut die Hälfte der Befragten $(52,3 \%)$ besitzt ein Abitur oder einen höheren Bildungsabschluss. Das durchschnittliche Haushaltsnettoeinkommen innerhalb der Stichprobe beträgt $€ 2.873$ (Standardabweichung (SD)=€ 1.572).

\subsection{Risikowahrnehmung und Schädigungspotential der Mykotoxine}

Es zeigt sich, dass $43,4 \%$ der befragten Eltern das Gesundheitsrisiko durch Mykotoxine für ihr Kind als sehr oder eher gering einschätzen. Etwa ein Viertel (26,8\%) geht von einem mittleren, ein weiteres Viertel $(26,4 \%)$ von einem eher hohen bis sehr hohen Risiko aus. Gar kein Gesundheitsrisiko durch Mykotoxine in Getreideprodukten sehen 3,4\% der Eltern (Abbildung 1). Gefragt nach dem Gesundheitsrisiko durch Mykotoxine für Erwachsene wird deutlich, dass die Befragten das Risiko für ihr Kind $(M=3,60, S D=1,47)$ im Durchschnitt signifikant höher einschätzen als das für Erwachsene $(M=3,22, S D=1,54, t(234)=4,7$, $\mathrm{p}<0,001, \mathrm{r}=0,29)$.

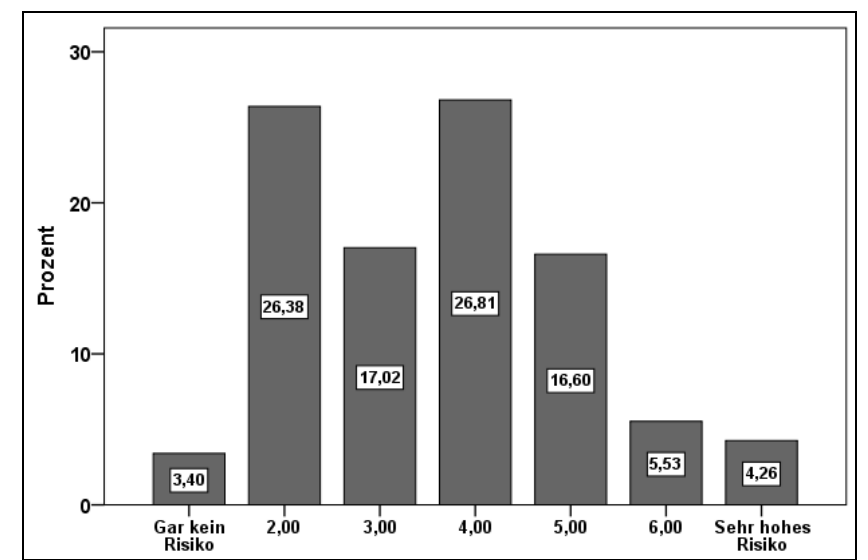

Abb. 1: Einschätzung des Gesundheitsrisikos durch Mykotoxine aus Elternsicht bezogen auf das eigene Kind in Deutschland

Quelle: Eigene Erhebung, 2012 
Bezüglich des Potentials der Mykotoxine zur Gesundheitsschädigung ist knapp ein Drittel der Eltern (27,8\%) der Auffassung, dass Mykotoxine die Gesundheit ihrer Kinder gar nicht oder nur geringfügig schädigen können. Demgegenüber stehen 53,5\%, die eine eher bis sehr ernsthafte Gesundheitsschädigung für möglich halten. Knapp 20\% $(18,8 \%)$ der Eltern gehen von einem mittleren Schädigungspotential der Mykotoxine aus. Das Alter der Kinder der Befragten führt nicht zu signifikanten Unterschieden in der Mykotoxin-Risikowahrnehmung oder in der Beurteilung des Schädigungspotentials der Mykotoxine bezogen auf das eigene Kind. Eltern von Jungen $(M=4,83, S D=1,68)$ schreiben Mykotoxinen jedoch im Durchschnitt ein signifikant größeres Potential zur Gesundheitsschädigung ihres Kindes zu als Eltern von Mädchen ( $\mathrm{M}=4,27, \mathrm{SE}=1,70, \mathrm{t}(231)=-2,56, \mathrm{p}<0,05, \mathrm{r}=0,17)$. Desweiteren sind die Befragten der Meinung, dass Mykotoxine die Gesundheit ihrer Kinder ernsthafter schädigen können $(\mathrm{M}=4,56, \mathrm{SD}=1,70)$ als die $\mathrm{Ge}$ sundheit von Erwachsenen $(M=4,06, S D=1,73, t(233)=7,18, p<0,001$, $\mathrm{r}=0,42)$.

\subsection{Aktuelles Risikomanagement und Verantwortlichkeit}

Das aktuelle gesetzliche Risikomanagement beurteilen $79,6 \%$ der Befragten als nur teilweise bis gar nicht ausreichend, um die Gesundheit von Kindern vor Mykotoxinen zu schützen. Nur 2,1\% der befragten Eltern sind hingegen der Auffassung, dass das gesetzliche Risikomanagement vollkommen ausreichend ist. Die übrigen 18,3\% bewerten das derzeitige Management des Mykotoxinrisikos als eher ausreichend, um einen umfassenden Gesundheitsschutz von Kindern zu gewährleisten. Die Verantwortung, die Gesundheit von Kindern vor Mykotoxinen zu schützen, liegt aus Sicht von etwa der Hälfte der Befragten $(48,9 \%)$ überwiegend oder sogar ausschließlich beim Staat. Gut ein Drittel $(36,2 \%)$ schreibt die Verantwortlichkeit zu gleichen Teilen den Eltern selbst und dem Staat zu. Nur 14,9\% der Befragten sehen die Verantwortung überwiegend oder ausschließlich bei den Eltern.

\subsection{Zahlungsbereitschaft für eine Risikoreduzierung}

Bezüglich der „Kinderweizenbrötchen“, die einen erhöhten Gesundheitsschutz vor Mykotoxinen gewährleisten, geben 13,4\% der befragten 
Eltern an, diese grundsätzlich nicht für ihre Kinder kaufen zu wollen. Hingegen äußern 27,2\% eine eindeutige Kaufabsicht. Mehr als die Hälfte $(59,5 \%)$ ist unsicher und antwortet, Kinderbrötchen „vielleicht" kaufen $\mathrm{zu}$ wollen. Es gibt einen signifikanten, positiven Zusammenhang zwischen der Einschätzung des Gesundheitsrisikos durch Mykotoxine und der Kaufbereitschaft $\left(\mathrm{r}_{\mathrm{s}}=0,14\right)$. Die durchschnittliche Zahlungsbereitschaft für ein herkömmliches Weizenbrötchen entspricht 32 Cent (SD=11 Cent). Die Eltern, die den Kauf eines „Kinderweizenbrötchens" nicht generell ablehnen, sind zu 97,8\% bereit, einen Aufpreis zwischen 5 Cent und 30 Cent zu zahlen (Abbildung 2). Die durchschnittliche absolute Mehrzahlungsbereitschaft für ein „Kinderweizenbrötchen", das nur so viele Mykotoxine enthält, dass ein Gesundheitsrisiko für Kinder ausgeschlossen werden kann, beträgt 13 Cent ( $\mathrm{SD}=8 \mathrm{Cent})$. Die durchschnittliche relative Mehrzahlungsbereitschaft für ein „Kinderweizenbrötchen“ beträgt 43,58\% (SD=31,47\%). Es gibt einen signifikant positiven, jedoch schwachen Zusammenhang zwischen der relativen Mehrzahlungsbereitschaft und der Einschätzung des Gesundheitsrisikos des eigenen Kindes durch Mykotoxine $\left(r_{s}=0,16\right)$. Gleiches gilt für die Beziehung zwischen der absoluten Mehrzahlungsbereitschaft und der Einschätzung des Mykotoxinrisikos für das eigene Kind $\left(r_{s}=0,16\right)$.

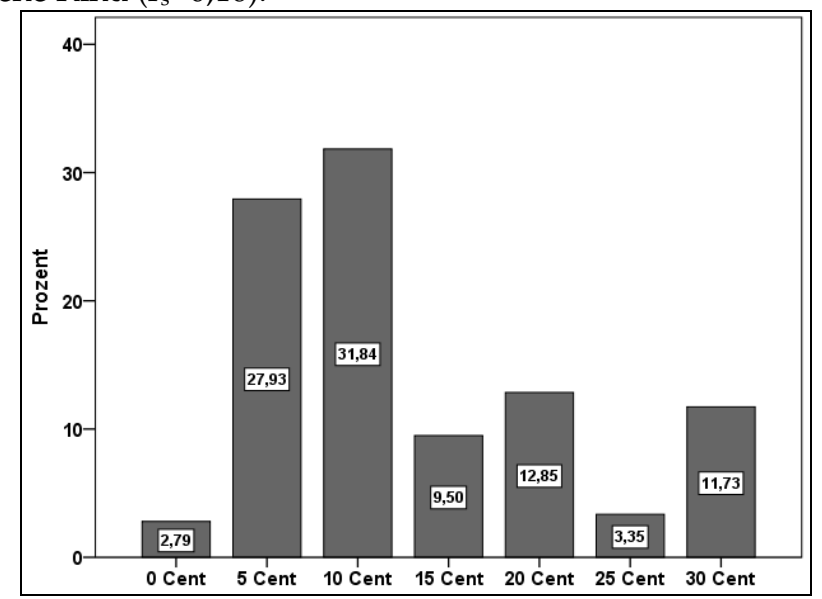

Abb. 2: Elterliche Mehrzahlungsbereitschaft für ein "Kinderweizenbrötchen", das nur so viele Mykotoxine enthält, dass ein Gesundheitsrisiko für Kinder ausgeschlossen werden kann

Quelle: Eigene Erhebung, 2012 
Es sind keine signifikanten Unterschiede in der Mehrzahlungsbreitschaft zwischen Eltern von Mädchen und Eltern von Jungen beobachtbar. Jedoch besitzen Eltern mit im oder nach dem Jahr 2007 geborenen Kindern im Durchschnitt eine signifikant höhere relative Mehrzahlungsbereitschaft $(\mathrm{Md}=0,39)$ als Eltern mit älteren Kindern $(\mathrm{Md}=0,33$, $\mathrm{U}=2939,50, \mathrm{p}<0,05, \mathrm{r}=0,15)$. Das Bildungsniveau der Eltern führt nicht $\mathrm{zu}$ signifikanten Unterschieden in der relativen und absoluten Mehrzahlungsbereitschaft für ein besonders sicheres „Kinderweizenbrötchen“. Gleiches gilt für das Haushaltsnettoeinkommen der Befragten.

\section{Diskussion}

Insgesamt zeigt der überwiegende Teil der befragten Eltern ein Problembewusstsein für die Kontamination von Getreideprodukten durch Mykotoxine. Dabei schätzen Eltern das konkrete Gesundheitsrisiko für ihr Kind geringer ein als das grundsätzliche Potential der Mykotoxine zur Gesundheitsschädigung ihres Kindes. Die befragten Eltern bewerten das Gesundheitsrisiko durch Mykotoxine für ihr Kind höher als das für Erwachsene, jedoch scheinen sie das Risiko tendenziell zu unterschätzen. So sehen einige Eltern überhaupt kein oder nur ein sehr geringes Gesundheitsrisiko für ihr Kind. Dieser Position muss, insbesondere da es sich bei Kindern um eine Risikogruppe handelt, aus Expertensicht zumindest für das Vorliegen einer chronischen Gesundheitsgefährdung widersprochen werden (RAUPACH und MARGGRAF, 2011, 196ff). Beachtenswert ist, dass das Alter der Kinder der Befragten keine Unterschiede in der Mykotoxin-Risikowahrnehmung bewirkt, obwohl kleine Kinder unter fünf bzw. sieben Jahren gefährdeter sind als ältere (CURTUI et al., 2006, 111ff; RAUPACH, 2012, 115ff). Hier könnte ein Ansatz für eine zielgruppenorientierte Risikokommunikation liegen.

Durch die aktuelle gesetzliche Regulierung wird die Gesundheit von Kindern nach Ansicht der Befragten nicht ausreichend vor Mykotoxinen geschützt. Dabei sieht der überwiegende Teil der Befragten den Staat in der Verantwortung, einen zuverlässigen Gesundheitsschutz von Kindern vor Mykotoxinen sicherzustellen. Dennoch werden vorrangig privat ausgerichtete Maßnahmen zur Reduzierung des Mykotoxinrisikos, wie das Angebot eines besonders sicheren „Kinderweizenbrötchens", tendenziell positiv bewertet. Der große Anteil an KaufUnentschlossenen deutet aber darauf hin, dass die Absatzchancen sol- 
cher kindergerechten Getreideprodukte stark von der Vermarktungsstrategie und einer angemessenen Aufklärung der Eltern über die Mykotoxinproblematik abhängen wird. Dass grundsätzlich eine Mehrzahlungsbereitschaft für kindergerechte Getreideprodukte besteht, konnte hier gezeigt werden. Im Fall des sicheren „Kinderweizenbrötchens" beträgt diese im Durchschnitt 13 Cent. Der hohe Anteil an KaufUnentschlossen könnte jedoch auf eine Überschätzung der Mehrzahlungsbereitschaft hindeuten (GROOTHIUS und WHITEHEAD, 2002). Ginge man davon aus, dass alle Kauf-Unentschlossen nicht bereit wären, einen Aufpreis für besonders sichere „Kinderweizenbrötchen“ zu zahlen, reduzierte sich die Mehrzahlungsbereitschaft auf 5 Cent. Es konnte kein signifikanter Zusammenhang zwischen der Zahlungsbereitschaft für eine Reduzierung des Gesundheitsrisikos durch Mykotoxine für das eigene Kind und dem Einkommen der befragten Eltern nachgewiesen werden. Dies liegt vermutlich an den geringen Kosten (Centbeträge), die mit einer Reduzierung des Gesundheitsrisikos durch den Kauf eines besonders sicheren „Kinderweizenbrötchens" verbunden sind. Aus diesem Ergebnis lässt sich ableiten, dass auch Eltern mit vergleichsweise geringem Einkommen in der Lage sind, einen durchschnittlichen Aufpreis von 13 Cent für ein sicheres Brötchen für ihre Kinder zu zahlen. Damit erscheint ein privater und eigenverantwortlicher Gesundheitsschutz von Kindern vor Mykotoxinen auch für einkommensschwächere Familien prinzipiell möglich. Es muss jedoch bedacht werden, dass in der vorliegenden Analyse die Zahlungsbereitschaft für eine Verbesserung des Gesundheitsschutzes von Kindern vor Mykotoxinen exemplarisch anhand eines einzelnen Produktes untersucht wurde.

Um einen vollständigen Gesundheitsschutz von Kindern vor Mykotoxinen zu erreichen, müsste jedoch der überwiegende Teil der Getreideprodukte, die ein Kind verzehrt, durch besonders sichere „Kindergetreideprodukte“ ersetzt werden. Entsprechend lägen die zusätzlichen Kosten, die mit einer langfristigen und wirkungsvollen Reduzierung des Gesundheitsrisikos verbunden sind, deutlich höher als der hier ermittelte Betrag. In nachfolgenden Studien sollte daher die monatliche elterliche Mehrzahlungsbereitschaft, bezogen auf den gesamten Getreideverzehr des Kindes, erhoben und bezüglich der Abhängigkeit vom Haushaltsnettoeinkommen der Familie analysiert werden. Sollte sich das hier gefundene Ergebnis bestätigen, bestünde in der Bereitstel- 
lung besonders sicherer „Kindergetreideprodukte“ eine adäquate Möglichkeit, den Gesundheitsschutz von Kindern vor Mykotoxinen auf freiwilliger Basis zu verbessern.

\section{Danksagung}

Die AutorInnen danken dem niedersächsischen Ministerium für Wissenschaft und Kultur für die Finanzierung der Studie (FAEN 3Projekt).

\section{Literatur}

BARTELS, G. und RODEMANN, B. (2003): Strategien zur Vermeidung von Mykotoxinen in Getreide. Gesunde Pflanzen, 55, 5, 125-135.

BMELV (Bundesministerium für Ernährung, Landwirtschaft und Verbraucherschutz) (2010): Besondere Ernte- und Qualitätsermittlung (BEE) 2010. Bonn.

Curtui, V., Brockmaeyer, A., Dietrich, R., KAPPEnstein, O., KlafFKE, H., Lepschy, J., MÄrtlbauer, E., Schneider, E., Seidler, C., Thielert, G., Usleber, E., WEBER, R. und WOLFF, J. (2006): Analytik und Vorkommen wichtiger Fusarientoxine. Münster: Landwirtschaftsverlag $\mathrm{GmbH}$.

FREESE, C. (2010): Empirische und experimentelle Analyse von Verbrauchereinstellungen gegenüber Mykotoxinrisiken und Lebensmittelkennzeichnungen. Dissertation an der Georg-August-Universität Göttingen. Göttingen.

Groothius, P. A. und WhiteheAD, J. C. (2002): Does don't know mean no? Analysis of 'don't know' responses in dichotomous choice contingent valuation questions. Applied Economics, 34, 1935-1940.

RAUPACH, K. und MARGGRAF, R. (2011): Mykotoxine als Gesundheitsrisiko: Laienund Expertensicht. Jahrbuch der Österreichischen Gesellschaft für Agrarökonomie, 20, 1, 191-200.

RAUPACH, K. (2012): Risiko- und Risikomanagement ausgewählter FusariumMykotoxine. Stuttgart: Ibidem-Verlag.

SCF (Scientific Commitee on Food) (1999): Opinion on Fusarium Toxins Part 1: Deoxynivalenol (DON). Brüssel.

VERORDNUNG (EG) Nr. 1881/2006 der Kommission vom 19. Dezember 2006 zu Festsetzung der Höchstgehalte für bestimmte Kontaminationen in Lebensmitteln. Amtsblatt der Europäischen Union L 346/5.

Anschrift der VerfasserInnen

Dipl. Sozw. Christine Niens und Prof. Dr. Rainer Marggraf Georg-August-Universität Göttingen Platz der Göttinger Sieben 5, 37073 Göttingen, Deutschland Tel.: +49(0)5514853

eMail: cniens@uni-goettingen.de 


\subsection{Parental risk perception of mycotoxins and risk reduction behaviour}

Niens, C., Strack, M. und Marggraf, R. (2013): Parental risk perception of mycotoxins and risk reduction behaviour. Angenommen zur Veröffentlichung im British Food Journal am 08.04.2013. The article is forthcoming in the "British Food Journal" (Emerald Group Publishing). 


\section{Diskussion und Ausblick}

Ziel der vorliegenden Arbeit war die kritische Auseinandersetzung mit dem aktuellen Mykotoxin-Risikomanagement, wobei der Schwerpunkt auf der Verbesserung des Gesundheitsschutzes von Kindern vor Deoxynivalenol (DON) lag. Zunächst wurde mittels einer Kosten-Nutzen-Analyse geprüft, ob ein umfassender Verbraucherschutz, der auch die Risikogruppe „Kind“ angemessen berücksichtigt, ökonomisch sinnvoll ist. Es zeigt sich, dass die Verbesserung des gesundheitlichen Verbraucherschutzes vor DON aus wohlfahrtsökonomischer Sicht vorteilhaft ist, da der resultierende volkswirtschaftliche Nutzen die volkswirtschaftlichen Kosten übersteigt. In Bezug auf die durchgeführte KostenNutzen-Analyse muss jedoch berücksichtigt werden, dass nur der intangible Nutzen einer Verbesserung des Verbraucherschutzes und die direkten Kosten der Landwirtschaft gegenübergestellt wurden. Die direkten Kosten der Landwirtschadt wurden in Form der variablen Kosten für die verstärkten Aufwendungen zur Vermeidung eines Fusarium-Befalls des Weizens ermittelt. Die Bestimmung des intangiblen Nutzens erfolgte über die Zahlungsbereitschaft der Konsumenten. Eine Erfassung weiterer direkter und indirekter Kosten- und Nutzeneffekte war aufgrund der Komplexität der Mykotoxinproblematik nicht umsetzbar. Die Komplexität der Mykotoxinproblematik für die ökonomische Bewertung einer Reduzierung des Gesundheitsrisikos durch DON besteht vor allem darin, dass zwischen der Ursache (überhöhte DON-Aufnahme) und der Wirkung (Gesundheitseffekt) zahlreiche intervenierende Größen wirksam sind, die eine eindeutige Identifikation von Kosten infolge gesundheitlicher Beeinträchtigungen der Konsumenten und Nutzen durch Vermeidung dieser Erkrankungen unmöglich machen.

Trotzdem ist davon auszugehen, dass weitere Kosten- und Nutzeneffekte für die ökonomische Bewertung eines umfassenden Verbraucherschutzes vor DON relevant sind. Zusätzlicher direkter Nutzen könnte vor allem aus der Vermeidung von Krankheitskosten resultieren. Aus Expertensicht wäre hier besonders mit Einsparungen für die Behandlung chronischer Erkrankungen, wie einer Schwächung des Immunsystems, zu rechnen (Raupach und Marggraf, 2011; SCF, 1999, 2002). Jedoch erscheint die Bestimmung vermiedener Krankheitskosten für chronische Gesundheitseffekte durch DON vor dem Hintergrund, dass die Herstellung klarer Ursache-Wirkungs-Beziehungen nicht möglich ist (vgl. Kapitel 3.2), kaum umsetzbar. 
Indirekte Nutzeneffekte einer Verbesserung des Verbraucherschutzes vor DON könnten aus einer Verringerung der Fehlzeiten von Kindern in der Schule resultieren. Da DON eine Schwächung des Immunsystems verursacht, in deren Folge sich die Anfälligkeit der Betroffenen für Infektionskrankheiten erhöht, kann eine überhöhte DON-Aufnahme ursächlich für eine beachtliche Zahl an Fehltagen in der Schule sein, wodurch sich die Bildungschancen der Kinder verschlechtern. Dies wirkt sich wiederum negativ auf das Humankapital einer Gesellschaft aus. Darüber hinaus könnten sich indirekte Nutzeneffekte aus der Vermeidung von Produktionsverlusten ergeben. Wenn die Anzahl DON-bedingter Erkrankungen bei Kindern reduziert wird, vermindert sich auch die Anzahl von Fehltagen der Eltern am Arbeitsplatz, weil diese seltener die Betreuung ihrer erkrankten Kinder übernehmen müssen. Da es jedoch nicht möglich ist, die Anzahl DON-bedingter Krankheitsfälle zu quantifizieren, können die indirekten Nutzeneffekte einer Vermeidung DON-bedingter Erkrankungen nicht bestimmt werden (vgl. Kapitel 3.2).

Indirekte Kosten eines umfassenden Verbraucherschutzes vor DON, die im Rahmen der durchgeführten Kosten-Nutzen-Analyse nicht berücksichtigt werden konnten, könnten durch die Notwendigkeit einer alternativen Verwendung von Weizenpartien, die den strengeren Qualitätsanforderungen nicht entsprechen, verursacht werden. Außerdem könnte der geringere Ertrag Fusarium-resistenter Weizensorten zu einem erhöhten Flächenbedarf für den Weizenanbau führen. Hierdurch würde weniger Anbaufläche für andere Getreidesorten zur Verfügungen stehen, was eine Erhöhung der Lebensmittelpreise, insbesondere für getreidehaltige Produkte, zur Folge haben könnte.

Bezüglich des in der Kosten-Nutzen-Analyse berücksichtigten intangiblen Nutzens einer Verbesserung des gesundheitlichen Verbraucherschutzes vor DON muss beachtet werden, dass sich die verwendete Zahlungsbereitschaft nicht ausschließlich auf den verbesserten Gesundheitsschutz von Kindern bezieht, sondern auf die Gesamtgruppe der Konsumenten. Da aber in erster Linie Kinder von einer Gesundheitsgefährdung durch DON betroffen sind, müsste eine Zahlungsbereitschaft explizit für eine Reduzierung des Gesundheitsrisikos von Kindern erhoben werden. Kinder können jedoch als die direkt Betroffenen aufgrund ihres kognitiven Entwicklungsstandes nicht direkt befragt werden. Stattdessen sollte die Erhebung der Zahlungsbereitschaft über die Eltern erfolgen (vgl. Niens und Marggraf, 2010). Aus der ökonomischen Literatur ist zu entnehmen, dass Eltern die Gesundheit ihrer Kinder höher 
bewerten als ihre eigene (z. B. Liu et al., 2000; Agee und Crocker, 2001; Dickie und Ulery, 2002; Hoffman, 2007). Vor diesem Hintergrund ist davon auszugehen, dass die Zahlungsbereitschaft für einen umfassenden Verbraucherschutz in der hier durchgeführten Analyse unterschätzt ist. Wäre die Abfrage der Zahlungsbereitschaft stärker mit dem Schutz von Kindergesundheit in Verbindung gebracht worden als es in der Studie von Freese (2010) der Fall war, wäre der intangible Nutzen einer Verbesserung des Verbraucherschutzes vor DON in der durchgeführten Kosten-Nutzen-Analyse vermutlich höher gewesen.

Im Rahmen der vorliegenden Dissertation wurde dem Erfordernis, eine kinderbezogene Zahlungsbereitschaft ermitteln zu müssen, mithilfe einer Elternbefragung zum Thema „Kinderernährung und Lebensmittelsicherheit in Deutschland“ nachgekommen. Auf die zahlreichen Schwierigkeiten, die mit der ökonomischen Bewertung von Kindergesundheit verbunden sind, wurde im Beitrag „Ökonomische Bewertung von Kindergesundheit in der Umweltpolitik. Aktuelle Ansätze und ihre Grenzen“ (Niens und Marggraf, 2010) ausführlich eingegangen. An dieser Stellte soll zusätzlich dargestellt werden, wie ausgewählten Problemen der Bestimmung einer kinderspezifischen Zahlungsbereitschaft im Rahmen der durchgeführten Elternbefragung begegnet wurde.

Die erste Schwierigkeit bei der Bestimmung kinderbezogener Daten resultiert aus dem Umstand, dass Kinder nicht direkt nach ihrer Zahlungsbereitschaft für eine Verringerung des Gesundheitsrisikos durch DON befragt werden können. Daher musste die Datenerhebung über Stellvertreter erfolgen. Entsprechend der Empfehlungen der U.S. Environmental Protection Agency (2003) und der Working Party on National Environmental Policy (2005) wurde die kinderspezifische Zahlungsbereitschaft für eine Reduzierung des Mykotoxinrisikos über die Eltern erfasst. Die Ermittlung einer Zahlungsbereitschaft über Stellvertreter ist jedoch mit verschieden Probleme verbunden. Zum einen bringt sie die Notwendigkeit mit sich, familiäre Entscheidungsprozesse berücksichtigen zu müssen (vgl. Kapitel 4.4 in Niens und Marggraf, 2010). Zum anderen sind bei der Erhebung kinderbezogener Zahlungsbereitschaften über die Eltern Verzerrungen infolge sozialer Erwünschtheit sehr wahrscheinlich, welche in der Regel zu Überschätzungen der ermittelten Werte führen.

In Bezug auf die Einbeziehung familiärer Entscheidungsprozesse stellt sich die Frage, wie diese angemessen berücksichtigt werden können. In der hier durchgeführten Befragung wurde dem Vorschlag von Bateman und Munro (2006) gefolgt, einen Verantwortlichen für 
den Bewertungsgestand zu identifizieren. Da es sich bei dem Gesundheitsrisiko durch DON um ein Lebensmittelrisiko handelt, muss für die Erhebung einer kinderbezogenen Zahlungsbereitschaft für eine Verbesserung des Verbraucherschutzes vor DON die Person identifiziert werden, die für den Lebensmitteleinkauf der Familie hauptverantwortlich ist. Hierfür enthielt der Fragebogen einen schriftlichen Hinweis, dass die Bearbeitung durch die Personen erfolgen sollten, die hauptverantwortlich für den Lebensmitteleinkauf der Familie ist. Zudem erfolgte die Identifizierung der oder des Hauptverantwortlichen für den Lebensmitteleinkauf zu Beginn der durchgeführten Studie mithilfe folgender Kontrollfrage: „Wer ist in Ihrer Familie für den Lebensmitteleinkauf hauptsächlich verantwortlich? (Ich; Jemand anderes; Jemand anderes und ich gleichermaßen). Insgesamt 97,9\% der befragten Eltern waren entweder allein $(n=166)$ oder in gleicher Weise wie jemand anderes $(n=66)$ für den Lebensmitteleinkauf der Familie verantwortlich.

Hinsichtlich der Kontrolle von Effekten sozialer Erwünschtheit wurden die Eltern vor der Bearbeitung des Discrete Choice Experiments schriftlich darauf hingewiesen, ihre finanzielle Situation zu berücksichtigen. Zusätzlich enthielt der Fragebogen den Hinweis, dass bei Wahl einer teuren Alternative weniger Geld für andere Dinge zur Verfügung steht. Weiterhin beinhaltete der verwendete Fragebogen eine Skala zu Messung sozialer Erwünschtheit (SES17, Stöber 1999). Die SES-17 misst mittels verschiedener Items die Tendenz eines Befragten sozial unerwünschtes, aber weit verbreitetes Verhalten zuzugeben. Das Ausmaß der Beeinflussung eines Befragten durch soziale Erwünschheitseffekte lässt sich über die Korrelation zwischen SES-17 und der Zahlungsbereitschaft ermitteln. Eine starke (positive) Korrelation zwischen dem SES-17 und Zahlungsbereitschaft lässt auf starke Beeinflussung des Antwortverhaltens schließen.

Neben den spezifischen Problemen, die bei der Erhebung kinderbezogener Daten über die Eltern auftreten können, gibt es auch weitere Störgrößen, die bei der Erfassung von Zahlungsbereitschaften Einfluss auf Reliabilität und Validität der Messung haben können. Die Reliabilität bezeichnet ein Maß für die Zuverlässigkeit der Messung. Die Validität gibt an, inwieweit das Messinstrument das Konstrukt messen konnte, was gemessen werden sollte.

Die Erhebung der Zahlungsbereitschaft für eine Verbesserung des Gesundheitsschutzes von Kindern vor DON erfolgt in der durchgeführten Elternbefragung unter anderem über den Kontingenten Bewertungsansatz. Die befragten Eltern wurden gebeten, ihre 
Mehrzahlungsbereitschaft für besonders sichere Getreideprodukte, welche sicher nur so viele Mykotoxine enthalten, dass ein Gesundheitsrisiko für Kinder ausgeschlossen werden kann, zu äußern. Um die Reliabilität des Messinstruments zu verbessern, erfolgte die Erfassung der elterlichen Mehrzahlungsbereitschaft über vier Items. Im Einzelnen wurde die Mehrzahlungsbereitschaft jeweils für ein Kinder-Weizenbrötchen, ein KinderMehrkornbrötchen, ein Kinder-Toastbrot und ein Kinder-Vollkornbrot erfragt. Die Mehrzahlungsbereitschaften eines Befragten bezüglich dieser vier Produkte wurden bei der späteren Auswertung jeweils addiert und gemittelt.

Durch die Erhebung der Zahlungsbereitschaft über mehrere Items können jedoch nicht alle Störgrößen, die zu Messfehlern bei der Datenerhebung führen können, kontrolliert werden. Bei der Erfassung der elterlichen Zahlungsbereitschaft für die Verbesserung des Gesundheitsschutzes ihrer Kinder ist neben den bereits erwähnten Effekten sozialer Erwünschtheit mit weiteren verzerrenden Einflüssen zu rechnen. Relevant für die durchgeführte Befragung sind vor allem der „Hypothetical Bias“ (Voelckner, 2006) und die „Informationsverzerrung“ (Bräuer und Suhr, 2005). Der „Hypothetical Bias“ beschreibt das Phänomen, dass Personen bereit sind, einen höheren Preis für ein Gut zu zahlen, wenn die Abfrage nur theoretisch erfolgt und der geäußerte Betrag nicht tatsächlich entrichtet werden muss. Damit hat der „Hypothetical Bias“ vermutlich eine Überschätzung der elterlichen Zahlungsbereitschaft für eine Verbesserung des Gesundheitsschutzes von Kindern vor DON verursacht. Weitere Messfehler könnten durch „Informationsverzerrung“ entstanden sein. „Informationsverzerrungen“ können auftreten, wenn die Befragten zur Bewertung eines Gutes Informationen benötigen, die ihnen während der Befragung gegeben werden (Bräuer und Suhr, 2005, S. 157). Durch die Art und Menge der Informationen können die Befragungsergebnisse beeinflusst werden, wobei die Richtung der Verzerrung (Überoder Unterschätzung der Zahlungsbereitschaft) offen ist. Da dem hier eingesetzten Fragebogen aufgrund des geringen Bekanntheitsgrades der Mykotoxinproblematik innerhalb der Bevölkerung ein Informationstext zu Mykotoxinen beigefügt wurde, muss mit „Informationsverzerrung“ gerechnet werden. Unklar ist jedoch, ob der Informationstext zu einer Über- oder Unterschätzung der elterlichen Zahlungsbereitschaft für eine Reduzierung des Mykotoxin-Risikos für Kinder geführt hat. Einerseits könnte die Information über ein potentielles Gesundheitsrisiko für Kinder durch Mykotoxine bewirkt haben, dass Eltern überhaupt bereit sind, einen Aufpreis für besonders sichere Kindergetreideprodukte zu 
zahlen. Andererseits könnte die Aussage, dass „[...] mit einer langfristig überhöhten Mykotoxin-Aufnahme bei Kindern nur sehr selten zu rechnen [ist]" zu einer Minderung der elterlichen Zahlungsbereitschaft beigetragen haben. Letztlich ist es wahrscheinlich, dass der Einfluss der oben genannten Störgrößen zu Verzerrungen der ermittelten Zahlungsbereitschaft für die Verbesserung des Gesundheitsschutzes von Kindern geführt hat, wobei sowohl Über- als auch Unterschätzungen denkbar sind.

Zusätzliche Messfehler könnten aus der Filterfrage ${ }^{12}$ resultieren, die der Abfrage der Mehrzahlungsbereitschaft für besonders sichere Kindergetreideprodukte vorausging. Infolge der Filterfrage haben nur die Eltern die Zahlungsbereitschaftsfragen beantwortet, die auch grundsätzlich oder zumindest vielleicht bereit wären, spezielle Kindergetreideprodukte für ihre Kinder zu kaufen. Es kann jedoch nicht ausgeschlossen werden, dass die Eltern, die den Kauf sicherer Kinder-Getreideprodukte ablehnen, zusätzliche Kosten für diese Produkte vorhergesehen haben. Träfe diese Vermutung zu, wäre die Anzahl der Eltern, die nicht bereit sind einen Aufpreis für die speziellen Kindergetreideprodukte zu zahlen, ohne eine Filterfrage höher gewesen. Somit kann die Tatsache, dass nur die Eltern nach einer Mehrzahlungsbereitschaft gefragt worden sind, die den Kauf der Kindergetreideprodukte nicht grundsätzlich ablehnen, eine Überschätzung der elterlichen Zahlungsbereitschaft verursacht haben.

Eine Möglichkeit, den Fehlerquellen bei der Erhebung der elterlichen Zahlungsbereitschaft für eine Reduzierung des Gesundheitsrisikos ihrer Kinder durch DON zu begegnen, könnte in der Angabe eines bestimmten Zahlungsbereitschafts-Intervalls liegen, das anstelle eines einzelnen Wertes betrachtet wird. Die obere Grenze dieses Zahlungsbereitschafts-Intervalls könnte der tatsächlich errechnete Wert von durchschnittlich 0,37 Euro (aufsummiert über die vier Produkte und gemittelt) darstellen. Die untere Grenze bildet der Wert von durchschnittlich 0,12 Euro (aufsummiert über die vier Produkte und gemittelt) der sich ergibt, wenn alle Nein- und Vielleicht-Käufer keine Mehrzahlungsbereitschaft für die besonders sicheren Kindergetreideprodukte besäßen. Die Zuschreibung einer Mehrzahlungsbereitschaft von 0 Euro für Vielleicht-Käufer könnte dabei Effekte sozialer Erwünschtheit und mögliche Informationsverzerrungen ausgleichen, sofern unterstellt wird,

\footnotetext{
${ }^{12}$ „Würden Sie spezielle Kinderbrote und Kinderbrötchen, die nur so viele Mykotoxine (Schimmelpilzgifte) enthalten, dass ein Gesundheitsrisiko für Kinder ausgeschlossen werden kann, für Ihr Kind kaufen?" (Nein; Vielleicht; Ja)
} 
dass diese Größen eine grundsätzliche Ablehnung der Produkte verhindern und die Bereitschaft, einen Aufpreis zu zahlen, hervorgerufen haben. Ein weiterer relevanter Wert innerhalb dieses Zahlungsbereitschafts-Intervalls stellt die durchschnittliche Höhe der Mehrzahlungsbereitschaft von 0,32 Euro (aufsummiert über die vier Produkte und gemittelt) dar. Dieser Wert entspricht der elterlichen Zahlungsbereitschaft für eine Reduzierung des Gesundheitsrisikos ihrer Kinder, wenn angenommen wird, dass die Eltern, die den Kauf spezieller Kindergetreideprodukte generell ablehnen, bereits zusätzliche Kosten antizipiert haben, die sie jedoch nicht zu tragen bereit sind.

In Bezug auf die hier durchgeführte Elternbefragung muss weiterhin mit Einschränkungen der externen Validität gerechnet werden. Die externe Validität gibt an, inwieweit die Untersuchungsergebnisse auf die Gesamtpopulation, für die die Ergebnisse Gültigkeit besitzen sollen, übertragbar sind. Damit eine Generalisierung der Analyseergebnisse auf die Gesamtpopulation möglich ist, sollten die untersuchten Personen möglichst repräsentativ für die betrachtete Bevölkerungsgruppe sein. Hierfür müssen bestimmte soziodemographische Merkmale der Stichprobe mit den Merkmalen der relevanten Gesamtpopulation übereinstimmen. Betrachtet man die externe Validität der Zahlungsbereitschaft, stellt das Einkommen der Befragten grundsätzlich eine relevante Größe für die Übertragbarkeit der Ergebnisse dar (Marggraf, 2005). Das durchschnittliche Haushaltsnettoeinkommen innerhalb der Stichprobe beträgt 2873 Euro monatlich (Standardabweichung (SD) = 1572 Euro; 93\% valide Antworten), bei einer mittleren Haushaltsgröße von 3,8 Mitgliedern. Das durchschnittliche Haushaltsnettoeinkommen von Paaren mit zwei Kindern in Deutschland beträgt 4429 Euro pro Monat (Statistisches Bundesamt, 2010). Damit liegt das durchschnittliche Haushaltsnettoeinkommen der befragten Personen deutlich unter dem bundesdeutschen Durchschnitt. Da von einem positiven Zusammenhang zwischen Zahlungsbereitschaft und Einkommen auszugehen ist, ist generell eine Unterschätzung des im Rahmen dieser Befragung ermittelten Wertes wahrscheinlich. Allerdings konnte im vorliegenden Fall kein signifikanter Zusammenhang zwischen dem Haushaltsnettoeinkommen der Befragten und der elterlichen Zahlungsbereitschaft für die Verbesserung des Verbraucherschutzes vor DON nachgewiesen werden. Folglich übt das Einkommen in dieser Untersuchung keinen beachtenswerten Einfluss auf die Höhe der elterlichen Zahlungsbereitschaft für besonders sichere Kindergetreideprodukte aus. Vor diesem Hintergrund erscheint die Tatsache, dass das 
Einkommen der befragten Eltern unter dem bundesdeutschen Durchschnitt liegt, für die externe Validität der Zahlungsbereitschaftsdaten weniger relevant.

Die beiden Dimensionen der elterlichen Mykotoxin-Risikowahrnehmung, Dread und Control, können 8,5\% der Variationen der elterlichen Zahlungsbereitschaft für eine Reduzierung des Gesundheitsrisikos ihrer Kinder mithilfe der besonders sicheren Kindergetreideprodukte erklären. Das bedeutet, dass 91,5\% unaufgeklärt bleiben. Ein theoretischer Ansatz, welcher einen hohen Wert für die Erklärung von gesundheitsbezogenem Verhalten besitzt, ist die Protection Motivation Theory (Maddux und Rogers, 1983). Entsprechend der Protection Motivation Theory ist die Motivation, die eigene Gesundheit zu schützen von vier Hauptfaktoren abhängig:

- Die wahrgenommene Ernsthaftigkeit einer Gefahr (severity)

- Die wahrgenommene Eintrittswahrscheinlichkeit oder persönliche Anfälligkeit (vulnerability)

- Die wahrgenommene Wirksamkeit des empfohlenen, vorbeugenden oder risikoreduzierenden Verhaltens (response efficacy)

- Die Überzeugung, das empfohlene, vorbeugende oder risikoreduzierende Verhalten auch persönlich ausführen zu können (self efficacy)

Die Motivation einer Person, die eigene Gesundheit zu schützen, resultiert aus der Abschätzung der Gefahr (threat appraisal) und der Bewertung der Möglichkeiten zur Gefahrenabwehr (coping appraisal). Für die Einschätzung der Gefahr (threat appraisal) sind die wahrgenommene persönliche Anfälligkeit (vulnerability) und die wahrgenommene Ernsthaftigkeit der Bedrohung (severity) ausschlaggebend. Die Bewertung der Möglichkeiten zur Gefahrenabwehr (coping appraisal) ist das Ergebnis der Einschätzung der Wirksamkeit des empfohlenen, vorbeugenden Verhaltens (response efficacy) und der Überzeugung, dieses Verhalten auch persönlich ausführen zu können (self efficacy). Grundsätzlich gilt dabei: je stärker die wahrgenommene Bedrohung (threat appraisal) und je positiver die Bewertung der Möglichkeiten zur Gefahrenabwehr (coping appraisal), desto größer ist die Motivation des Betroffenen, die eigene Gesundheit zu schützen. Dabei ist jedoch zu beachten, dass die Hauptkomponenten der Protection Motivation Theory "serverity“, „vulnerability“, „self efficacy“ und „response efficacy“ nicht multiplikativ, sondern subadditiv verknüpft sind. Das bedeutet, für eine starke Verhaltensintention ist es ausreichend, wenn zwei der Hauptkomponenten der Protection Motivation Theory stark ausgeprägt sind. Durch 
die starke Ausprägung einer dritten Variable verstärkt sich die Verhaltensintension nicht zusätzlich (kompensatorische Interaktion) (Maddux und Rogers, 1983, S. 476). Allerdings führen Personen bei starker Ausprägung der „Threat“ Variablen nur dann eine Handlung aus, die zum Schutz ihrer Gesundheit beiträgt, wenn sie auch glauben, zur Gefahrenabwehr (coping appraisal) in der Lage zu sein. Ist das nicht der Fall, kann sich ein gesundheitsschädliches Verhalten noch verstärken (Boomerang-Effekt) (Rogers und Prentice-Dunn, 1997, S. 119).

Der Kauf besonders sicherer Kindergetreideprodukte und die Höhe der Mehrzahlungsbereitschaft für diese Produkte kann als Ausdruck der elterlichen Motivation zum Schutz der Gesundheit ihrer Kinder vor Mykotoxinen angesehen werden. In zukünftigen Analysen sollten daher die Hauptkomponenten der Protection Motivation Theory bei der Vorhersage und Erklärung der elterlichen Zahlungsbereitschaft für die Reduzierung des Mykotoxin-Risikos berücksichtigt werden. Es ist zu erwarten, dass mit zunehmender Ausprägung der „Threat“ und/oder „Coping“ Variablen auch die Zahlungsbereitschaft der Eltern für die besonders sicheren Kindergetreideprodukte zunimmt. Die „Threat“ Variablen (severity und vulnerability) ähneln inhaltlich stark den Dimensionen der elterlichen Mykotoxin-Risikowahrnehmung "Dread“ und "Control“. Eine erhöhte Erklärungskraft des Modells ist daher vor allem von der "Coping“ Variable „response efficacy“ zu erwarten. Allerdings könnte mithilfe einer Mediationsanalyse untersucht werden, ob die „Threat“ Variablen (severity und vulnerability) zusätzlich zur Erklärung der elterlichen Zahlungsbereitschaft für die besonders sicheren Kindergetreideprodukte beitragen können. Denkbar wäre, dass die Dimension „Dread“ zwischen der wahrgenommenen Ernsthaftigkeit der Bedrohung (severity) und der elterlichen Zahlungsbreitschaft für eine Reduzierung des Mykotoxinrisikos vermittelt. Hingegen könnte die Dimension "Control“ zwischen der wahrgenommenen persönlichen Anfälligkeit (vulnerability) und der elterlichen Zahlungsbereitschaft vermitteln.

Für weitere Forschungsaktivitäten bezüglich der Verbesserung des gesundheitlichen Verbraucherschutzes vor DON mithilfe spezieller Kindergetreideprodukte ist besonders die Frage der Produktkennzeichnung interessant. In Betracht käme eine Kennzeichnung, die zeigt, dass die speziellen Kindergetreideprodukte entsprechend der Diätverordnung für Baby- und Kleinkindernahrung (DiätV, 2010, §14) produziert worden sind, oder ein spezielles 
Mykotoxin-Label (Freese, 2010). Zu untersuchen wäre, welche Alternative von Eltern bevorzugt wird, beziehungsweise wie sich die unterschiedlichen Kennzeichnungen auf die Zahlungsbereitschaft für die besonders sicheren Kindergetreideprodukte auswirken. Zudem wäre es sinnvoll, zu prüfen, ob ein zusätzliches Bio-Siegel die elterliche Kauf- und Zahlungsbereitschaft für die besonders sicheren Kindergetreideprodukte erhöht. Da eine Verbesserung des gesundheitlichen Verbraucherschutzes durch die generelle Senkung der Mykotoxin-Grenzwerte vor dem Hintergrund des ALARA-Prinzips („as low as reasonable achievable“, VO (EG) Nr. 1881/2006) kaum umsetzbar ist, erscheint weitere Forschung zur Akzeptanz der besonders sicheren Kindergetreideprodukte besonders lohnenswert. 


\section{Literatur II}

Agee, M. D. und. Crocker T. D. (2001): Smoking parents' valuations of own and children's health. Presented at the Association of Environmental and Resource Economists Conference, "Assessing and managing environmental and public health risks", Bar Harbor, Maine, 13-15 June.

Bateman, I. J. und Munro, A. (2006): Household versus individual valuation: What's the difference? URL: http://www.oecd.org/dataoecd/1/29/37585613.pdf (15.08.2009)

Bräuer, I. und Suhr, A. (2005): Ergebnisse von Zahlungsbereitschaftsanalysen - Interpretation und Verwendung. In: Marggraf, R. (Editor). Ökonomische Bewertung bei umweltrelevanten Entscheidungen. Einsatzmöglichkeiten von Zahlungsbereitschaftsanalysen in Politik und Verwaltung, Metropolis-Verlag, Marburg, S. 149-183.

DiätV (Verordnung über Diätische Lebensmittel, Diätverordnung) (2010). URL: http://www.gesetze-im-internet.de/bundesrecht/di_tv/gesamt.pdf (29. 08. 2012).

Dickie, M., und Ulery, V. L. (2002): Parental altruism and the value of avoiding acute illness: Are kids worth more than parents? Working paper, Department of Economics, University of Central Florida, Orlando, December.

Freese, C. (2010): Empirische und experimentelle Analyse von Verbrauchereinstellungen gegenüber Mykotoxinrisiken und Lebensmittelkennzeichnungen. URL: http://resolver.sub.uni-goettingen.de/purl/?webdoc-2632 (16. 07. 2011).

Hoffmann, S. (2007): Since children are not little adults-socially-What's an environmental economist to do? Duke Environmental Law \& Policy Forum 17 (2): 209-232.

Liu, J.-T., Hammitt, J.K., Wang, J.-D. und Liu, J.-L. (2000): Mother's willingness to pay for her own and her child's health: A contingent valuation study in Taiwan. Health Economics 9 (4): 319-326.

Maddux, J. E. und Rogers, R. W. (1983): Protection Motivation Theory and Self-Efficacy: A Revised Theory of Fear Appeals and Attitude Change. Journal of Experimental Social Psychology 19: 468-479. 
Marggraf, R. (2005): Ökonomische Grundlagen der Umweltbewertung. In Marggraf, R. (Editor). Ökonomische Bewertung bei umweltrelevanten Entscheidungen. Einsatzmöglichkeiten von Zahlungsbereitschaftsanalysen in Politik und Verwaltung. Metropolis-Verlag, Marburg, S. 61-83.

Niens, C. und Marggraf, R. (2010): Ökonomische Bewertung von Kindergesundheit in der Umweltpolitik. Aktuelle Ansätze und ihre Grenzen. Diskussionspapiere des DARE. URL: https://www.econstor.eu/dspace/bitstream/10419/40954/1/636384217.pdf (17.04.2013).

Raupach, K. und Marggraf, R. (2011): Mykotoxine als Gesundheitsrisiko: Laien- und Expertensicht. Jahrbuch der Österreichischen Gesellschaft für Agrarökonomie - Band 20 (1): 191-200. Facultas Verlag, Wien.

Rogers, R.W. und Prentice-Dunn, S. (1997): Protection Motivation Theory. Handbook of Health Behaviour Research I. Plenum Press, New York.

SCF (Scientific Committee on Food) (1999): Opinion of the Scientific Committee on Food on Fusarium toxins. Part 1: Deoxynivalenol (DON) (2. December 1999).

SCF (Scientific Committee on Food) (2002): Opinion of the Scientific Committee on Food on Fusarium toxins. Part 6: Group evaluation of T-2 toxin, HT-2 toxin, Nivalenol and Deoxynivalenol (adopted on 26 February 2002).

Statistisches Bundesamt (2010): „Wirtschaftsrechnungen, Einkommens- und Verbraucherstichprobe, Einkommen und Ausgaben privater Haushalte 2008“, Fachserie 15 Heft 4, Wiesbaden, S. 40.

Stöber, J. (1999): Die Soziale-Erwünschheits-Skala-17 (SES-17): Entwicklung und erste Befunde zu Reliabilität und Validität. Diagnostica 45 (4): 173-177.

U.S. Environmental Protection Agency (U.S. EPA), Office of Children's Health Protection; Office of Policy Economics and Innovation; National Center for Environmental Economics (2003): Children's Health Valuation Handbook. EPA 100-R-03-003. Washington, D.C.

Verordnung (EG) Nr. 1881/2006 der Kommission vom 19. Dezember 2006 zur Festsetzung der Höchstgehalte für bestimmte Kontaminanten in Lebensmitteln. 
Voelckner, F. (2006): An empirical comparison of methods for measuring consumers' willingness to pay. Market Letter 17: 137-149.

Working Party on National Environmental Policy (2005): The valuation of environmental health risks to children: Methodological and Policy Issues, ENV/EPOC/WPNEP (2004) 15/FINAL, OECD, URL: http://www.oecd.org/fr/environnement/ outilsetevaluationdespolitiquesdelenvironnement/35381312.pdf (15.01.2012). 


\section{Anhang}

Fragebogen zur Elternbefragung mit dem Titel „Befragung zur Kinder-Ernährung und Lebensmittelsicherheit in Deutschland: Schwerpunkt Brot und Backwaren“, in Göttingen und Hannover (Niedersachsen, Deutschland) im Januar und Februar 2012. 
G- GeORG-AuguSt-UniVERSITÄT

\section{Befragung zur}

\section{Kinder-Ernährung und}

\section{Lebensmittelsicherheit in Deutschland}

\section{Schwerpunkt Brot und Backwaren}

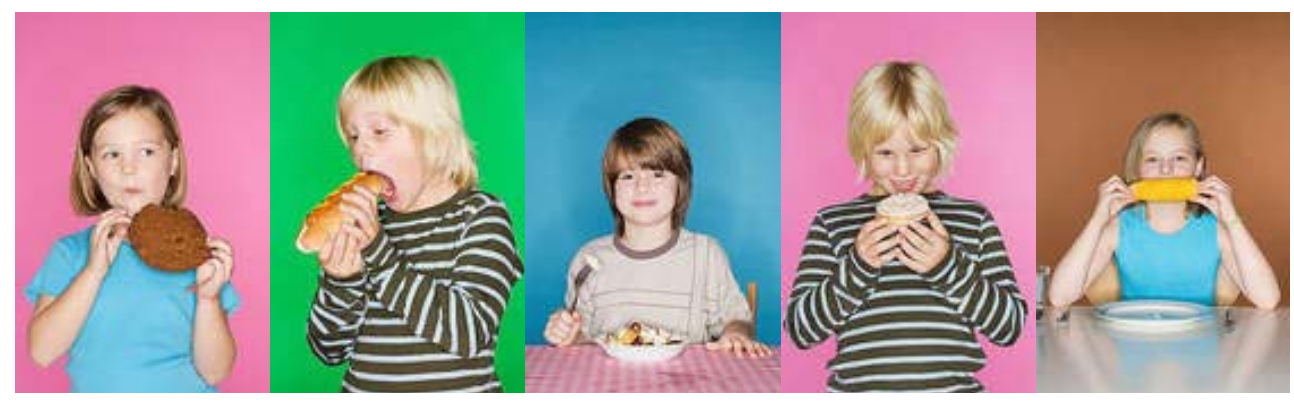




\section{Hinweise zum Ausfüllen des Fragebogens}

1. Der Fragebogen soll bitte von der Person ausgefüllt werden, die mit dem Kind in einem Haushalt lebt und normalerweise den Lebensmitteleinkauf für die Familie erledigt.

2. In diesem Fragebogen wird öfter von „Ihrem Kind“ gesprochen. Falls Sie mehrere Kinder haben, ist mit „Ihrem Kind“ immer das Kind gemeint, das zwischen 2001 und 2008 geboren worden ist. Haben Sie mehrere Kinder, die zwischen 2001 und 2008 geboren worden sind, entscheiden Sie sich jetzt bitte für eines dieser Kinder. Denken Sie bei der Beantwortung von Fragen zu „Ihrem Kind“ bitte immer an dieses bestimmte Kind. Denken Sie bitte bei allen Fragen zu „Ihrem Kind“ immer an das gleiche Kind.

3. Die Beantwortung des Fragerbogens erfolgt anonym. Das heißt, niemand weiß später, dass Sie diesen Fragebogen ausgefüllt haben.

4. Die Beantwortung dauert etwa 30-40 Minuten.

5. Es gibt keine falschen Antworten. Uns interessiert Ihre persönliche Meinung!

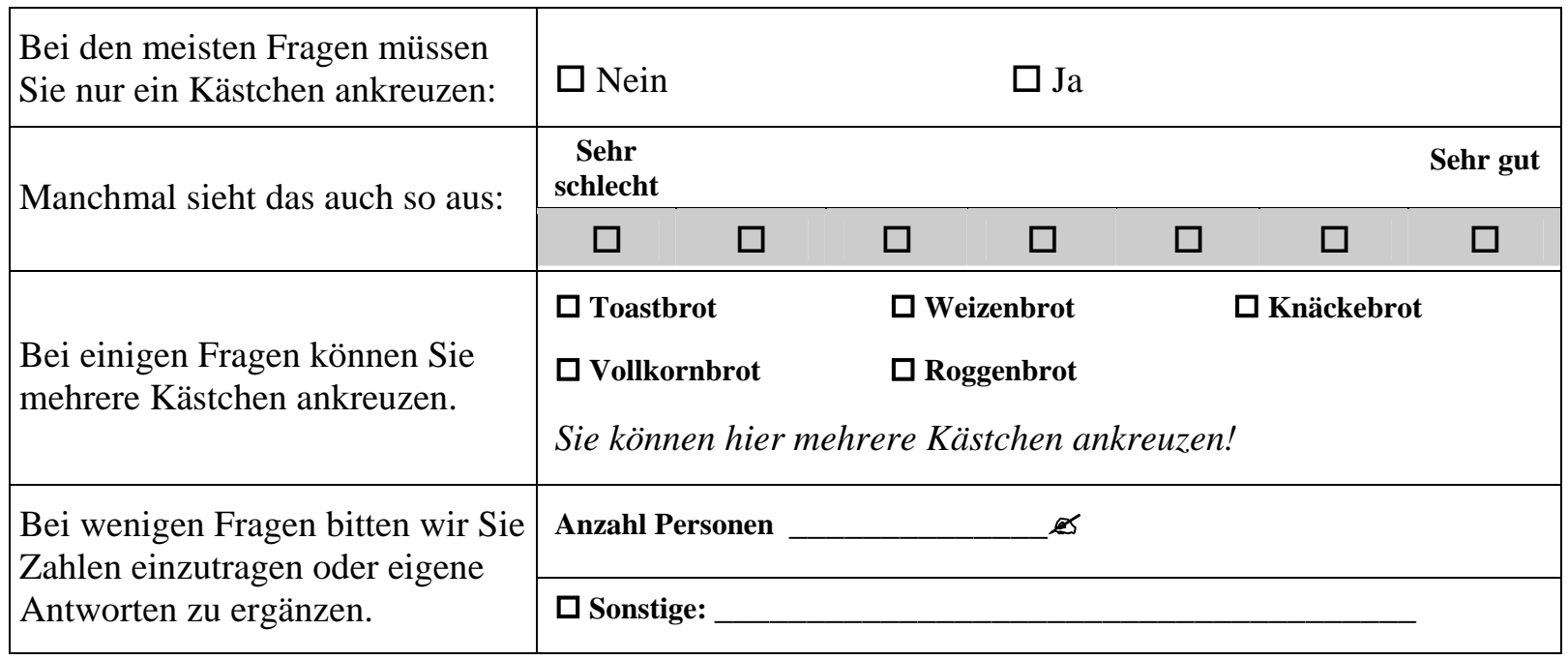

6. Bitte beantworten Sie den Fragebogen in der vorgegebenen Reihenfolge und lesen Sie sich die Informationstexte aufmerksam durch.

7. Bitte beantworten Sie unbedingt alle Fragen. Überspringen Sie Fragen nur, wenn Sie darauf hingewiesen werden.

8. Bitte geben Sie den ausgefüllten Fragebogen im beiliegenden Rückumschlag bis zum 10.02.2012 wieder bei den Erziehern/-innen Ihres Kindes ab!

Falls Sie Fragen haben, können Sie sich gerne an uns wenden:

Frau Dipl. Sozw. Christine Niens

Tel: 0551394853

E-mail: cniens@uni-goettingen.de

\section{Herr Prof. Dr. Rainer Marggraf}

Tel: 0551394829

E-mail: rmarggr@uni-goettingen.de 


\section{Fragen zum Lebensmitteleinkauf}

1. Wer ist in Ihrer Familie für den Lebensmitteleinkauf hauptsächlich verantwortlich?

$\square$ Ich $\quad \square$ Jemand anderes $\quad \square$ Jemand anderes und ich gleichermaßen

2. Wo kaufen Sie normalerweise Brot und Backwaren ein? Sie können hier mehrere Kästchen ankreuzen!

Bäckerei

$\square$ Bio-Laden/Bio-Bäcker

Selbstbedienungsbäcker

$\square$ Supermarkt wie Edeka, Rewe

$\square$ Discounter wie Lidl, Aldi

Backe selbst

\section{Fragen zu Ihrer Meinung über die Lebensmittelsicherheit in Deutschland}

3. Inwieweit stimmen Sie folgenden Aussagen zur Lebensmittelsicherheit in Deutschland zu?

$\begin{array}{ccc}\text { Bitte machen Sie in jeder Zeile ein Kreuz! } & \text { Stimme } & \text { gar } \\ \text { nicht zu } & \text { Stimme } \\ \text { voll und } \\ \text { ganz zu }\end{array}$

$\begin{array}{lllllll}\text { Bei uns kann man alles ohne Bedenken essen, } & \square & \square & \square & \square & \square & \square\end{array}$

In Deutschland sollten mehr

Lebensmittelkontrollen durchgeführt werden, auch wenn dadurch die Preise steigen.

\begin{tabular}{|c|c|c|c|c|c|c|c|}
\hline $\begin{array}{l}\text { Das Gerede über Rückstände in unseren } \\
\text { Nahrungsmitteln macht den Verbrauchern unnötig } \\
\text { Angst. }\end{array}$ & $\square$ & $\square$ & $\square$ & $\square$ & $\square$ & $\square$ & $\square$ \\
\hline $\begin{array}{l}\text { Wahrscheinlich wurden einige } \\
\text { Lebensmittelskandale noch gar nicht entdeckt. }\end{array}$ & $\square$ & $\square$ & $\square$ & $\square$ & $\square$ & $\square$ & $\square$ \\
\hline Es gibt nicht genug Lebensmittelkontrollen. & $\square$ & $\square$ & $\square$ & $\square$ & $\square$ & $\square$ & $\square$ \\
\hline
\end{tabular}

4. Hier sind einige Lebensmittel aufgelistet, die von Kindern regelmäßig verzehrt werden. Bitte geben Sie an, ob Sie wegen der Möglichkeit einer Belastung mit Pflanzenschutzmittel-Rückständen oder Schimmelpilzgiften gesundheitliche Bedenken hinsichtlich Ihres Kindes haben.

\begin{tabular}{|c|c|c|c|c|c|c|c|}
\hline Bitte machen Sie in jeder Zeile ein Kreuz! & $\begin{array}{c}\text { Gar } \\
\text { keine } \\
\text { Bedenken }\end{array}$ & & & & & & $\begin{array}{c}\text { Sehr } \\
\text { große } \\
\text { Bedenken }\end{array}$ \\
\hline $\begin{array}{l}\text { Pflanzenschutzmittel-Rückstände in frischem } \\
\text { Brot }\end{array}$ & $\square$ & $\square$ & $\square$ & $\square$ & $\square$ & $\square$ & $\square$ \\
\hline $\begin{array}{l}\text { Pflanzenschutzmittel-Rückstände in frischem } \\
\text { Obst }\end{array}$ & $\square$ & $\square$ & $\square$ & $\square$ & $\square$ & $\square$ & $\square$ \\
\hline $\begin{array}{l}\text { Pflanzenschutzmittel-Rückstände in Baby- } \\
\text { und Kleinkindernahrung }\end{array}$ & $\square$ & $\square$ & $\square$ & $\square$ & $\square$ & $\square$ & $\square$ \\
\hline Schimmelpilzgifte in frischem Brot & $\square$ & $\square$ & $\square$ & $\square$ & $\square$ & $\square$ & $\square$ \\
\hline Schimmelpilzgifte in frischem Obst & $\square$ & $\square$ & $\square$ & $\square$ & $\square$ & $\square$ & $\square$ \\
\hline
\end{tabular}

Schimmelpilzgifte in Baby- und

Kleinkindernahrung 
5. Wie stark sind Sie über die Lebensmittelsicherheit in Deutschland insgesamt verunsichert?

Gar nicht

verunsichert $\square$

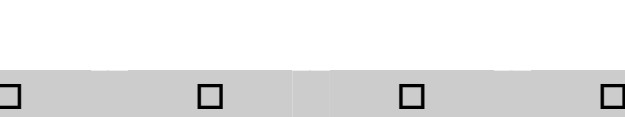

Sehr verunsichert

6. Wie viel Vertrauen haben Sie in die Sicherheit unserer Nahrungsmittel?

Gar kein

Vertrauen $\square$

\author{
$\square \quad \square$
}

\author{
$\square$
}

Völliges

Vertrauen

\section{Allgemeine Fragen zu Ihrem Kind und zu Ihrer Familie}

Bei den folgenden Fragen wird immer von „Ihrem Kind“ gesprochen. Wenn Sie mehrere Kinder haben, entscheiden Sie sich bitte für ein Kind, das zwischen 2001 und 2008 geboren ist. Bitte denken Sie bei der Beantwortung der Fragen immer an dieses bestimmte Kind.
7. Ihr Kind ist ein...
$\square$ Mädchen
$\square$ Junge

8. In welchem Jahr ist Ihr Kind geboren? 20

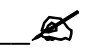

9. Wie würden Sie den Gesundheitszustand Ihres Kindes im Allgemeinen beschreiben? Sehr schlecht $\square$

$\square$

$\square$

$\square \quad \square$

$\square$ Sehr gut

10. Leidet Ihr Kind unter einer Erkrankung, die es erfordert, dass Sie besonders auf seine Ernährung achten (z. B. Lebensmittelallergien, Diabetes etc.)?

Nein

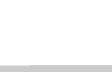

$\square \quad$ Sehr stark

$\square$


12. Einige Eltern haben die Möglichkeit etwas für „fremde“ Kinder zu tun, andere nicht. Wie oft im Jahr...

....spenden Sie gewöhnlich für Kinderhilfsprojekte (Geld oder

Sachspenden wie Spielzeug im Mindestwert von 5€)?

etwa mal pro Jahr

....arbeiten Sie gewöhnlich ehrenamtlich für Kinder (z. B. in

Betreuungseinrichtungen, Kindertafeln, als „Lesemutter/ -vater“,

Trainer im Verein etc.)?

etwa mal pro Jahr

Gerne können Sie hier Anmerkungen zu Fr. 12 hinterlassen:

Fragen zur Sicherheit von Getreideprodukten

Bitte lesen Sie den Informationstext aufmerksam durch!

Wichtige

Information

für Eltern!

\section{Getreideprodukte können unsichtbare Schimmelpilzgifte enthalten!}

Getreidehaltige Lebensmittel liefern wichtige Inhaltsstoffe, die Kinder für eine gesunde Entwicklung benötigen. Getreideprodukte können jedoch auch Schimmelpilzgifte enthalten, die so genannten „Mykotoxine“. Die Mykotoxine (Schimmelpilzgifte) werden von Pilzen gebildet, die das Getreide bereits auf dem Feld befallen. Die Verbreitung des Pilzes im Getreide und die Bildung der Mykotoxine (Schimmelpilzgifte) sind stark vom Wetter abhängig. Daher schwankt die Belastung der Lebensmittel von Jahr zu Jahr. Eine völlige Vermeidung der Mykotoxine (Schimmelpilzgifte) ist bislang nicht möglich.

Mykotoxine (Schimmelpilzgifte) werden regelmäßig in Weizenprodukten nachgewiesen. Sie sind für den Verbraucher nicht zu sehen, zu riechen oder zu schmecken. Zudem sind sie sehr hitzestabil und werden somit durch Backen nicht zerstört. Daher können die Mykotoxine (Schimmelpilzgifte) auch in frischem Brot enthalten sein, ohne dass Schimmelstellen direkt erkennbar sind.

Die Wirkung der Mykotoxine (Schimmelpilzgifte) auf die menschliche Gesundheit ist noch nicht vollständig erforscht. Fest steht jedoch, dass täglich eine begrenzte Menge an Mykotoxinen (Schimmelpilzgiften) aufgenommen werden kann, ohne dass ein Gesundheitsrisiko besteht. Sicher ist aber auch, dass ein häufig in Weizen vorkommendes Mykotoxin eine Schwächung des Immunsystems verursacht, wenn es längerfristig in zu großen Mengen aufgenommen wird. 
Um die Gesundheit der Verbraucher zu schützen, wurden Grenzwerte für die maximale Belastung von Getreideprodukten mit Mykotoxinen (Schimmelpilzgiften) festgelegt. Diese Grenzwerte schützen zuverlässig die Gesundheit von Erwachsenen. Einen 100\%igen Gesundheitsschutz für Kinder können sie aber nicht garantieren. Würden alle Getreideprodukte die gesetzlich maximal zulässige Menge an Mykotoxinen (Schimmelpilzgiften) enthalten, könnten Kinder erheblich mehr Mykotoxine aufnehmen, als aus medizinischer Sicht unbedenklich ist. In der Realität enthalten die meisten Getreideprodukte aufgrund freiwilliger Grenzwertunterschreitungen der Lebensmittelproduzenten aber viel weniger Mykotoxine (Schimmelpilzgifte) als erlaubt. Daher ist mit einer langfristig überhöhten Mykotoxin-Aufnahme bei Kindern nur sehr selten zu rechnen. Sicher ausschließen, dass einige Kinder mehr als eine unbedenkliche Menge an Mykotoxinen (Schimmelpilzgiften) aufnehmen, kann man derzeit jedoch nicht.

Jetzt geht es darum, wie Sie das Gesundheitsrisiko durch Mykotoxine (Schimmelpilzgifte) einschätzen. Wenn dabei von „Ihrem Kind“ gesprochen wird und Sie mehrere Kinder haben, denken Sie bei der Beantwortung bitte wieder an das Kind (geboren 2001-2008), auf das Sie sich auch bei den Fragen 7 bis 11 bezogen haben.

Bitte beachten Sie auch: Es geht uns nicht um Fachwissen. Wir interessieren uns für Ihre persönliche Meinung. Daher gibt es keine falschen Antworten!

Bitte machen Sie in jeder Zeile ein Kreuz! $\quad \begin{gathered}\text { Gar } \\ \text { kein }\end{gathered} \quad \begin{array}{r}\text { Sehr } \\ \text { hohes }\end{array}$

Wie hoch schätzen Sie das Gesundheitsrisiko durch Mykotoxine (Schimmelpilzgifte) in Getreideprodukten für Ihr Kind ein?

Wie hoch schätzen Sie das Gesundheitsrisiko durch Mykotoxine (Schimmelpilzgifte) in Getreideprodukten für Kinder im Allgemeinen in Deutschland ein?

Wie hoch schätzen Sie das Gesundheitsrisiko durch Mykotoxine (Schimmelpilzgifte) in Getreideprodukten für Erwachsene in Deutschland ein?

Bitte machen Sie in jeder Zeile ein Kreuz! Gar nich

Was meinen Sie, wie ernsthaft können Mykotoxine (Schimmelpilzgifte) die Gesundheit Ihres Kindes schädigen?

Was meinen Sie, wie ernsthaft können Mykotoxine (Schimmelpilzgifte) die Gesundheit von Kindern im Allgemeinen schädigen?

Was meinen Sie, wie ernsthaft können Mykotoxine (Schimmelpilzgifte) die Gesundheit von Erwachsenen schädigen? 
Wie besorgt sind Sie über ein mögliches Gesundheitsrisiko durch Mykotoxine (Schimmelpilzgifte) für Kinder im Allgemeinen?

Wie besorgt sind Sie über ein mögliches Gesundheitsrisiko durch Mykotoxine (Schimmelpilzgifte) für Erwachsene?

16. Was meinen Sie, wie verbreitet sind in Deutschland Getreideprodukte, die Mykotoxine (Schimmelpilzgifte) enthalten?

17. Für wie wahrscheinlich halten Sie es, dass die Gesundheit Ihres Kindes irgendwann durch Mykotoxine (Schimmelpilzgifte) aus Getreideprodukten geschädigt wird?

$\begin{array}{ccccccc}\begin{array}{c}\text { Sehr } \\ \text { unwahr- } \\ \text { scheinlich }\end{array} & & & & & \begin{array}{c}\text { Sehr wahr- } \\ \text { scheinlich }\end{array} \\ \square & \square & \square & \square & \square & \square & \square\end{array}$

\section{Bitte bedenken Sie: Es geht uns nicht um Fachwissen. Ihre persönliche Meinung ist gefragt. Daher gibt es keine falschen Antworten!}

18. Was meinen Sie, wie viele Kinder sind in Deutschland von einer Gesundheitsgefährdung durch Mykotoxine (Schimmelpilzgifte) aus Getreideprodukten betroffen?

Kein Kind

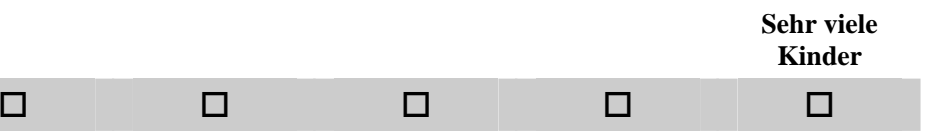

19. Was meinen Sie, wie viel wissen Wissenschaftler über mögliche Gesundheitsrisiken für Kinder durch Mykotoxine (Schimmelpilzgifte)?

Gar nichts

Alles

$\square$

$\square \quad \square$

$\square$

$\square$

20. Wie einfach ist es für Sie zu sagen, ob ein Lebensmittel, das Ihr Kind isst, Mykotoxine (Schimmelpilzgifte) enthält?

$\begin{array}{cccccc}\begin{array}{c}\text { Kann ich nie } \\ \text { sagen }\end{array} & & & \begin{array}{c}\text { Kann ich } \\ \text { immer sagen }\end{array} \\ \square & \square & \square & \square & \square & \square\end{array}$


21. Wie viel Kontrolle haben Eltern darüber, ob ihre Kinder Mykotoxine (Schimmelpilzgifte) über die Nahrung aufnehmen?

\section{Bitte bedenken Sie: Es geht uns nicht um Fachwissen. Ihre persönliche Meinung ist gefragt. Daher gibt es keine falschen Antworten!}

22. Was meinen Sie, können Mykotoxine schon in kleinsten Mengen schädlich für Ihr Kind sein, oder können Mykotoxine erst in großen Mengen schädlich für Ihr Kind sein?

$\begin{array}{cccccc}\begin{array}{c}\text { In kleinsten } \\ \text { Mengen } \\ \text { schädlich }\end{array} & & & & \begin{array}{c}\text { In großen } \\ \text { Mengen } \\ \text { schädlich }\end{array} \\ \square & \square & \square & \square & \square & \square\end{array}$

23. Was meinen Sie, ist Ihr Kind verglichen mit anderen Kindern mehr oder weniger durch Mykotoxine (Schimmelpilzgifte) in Getreideprodukten gefährdet?

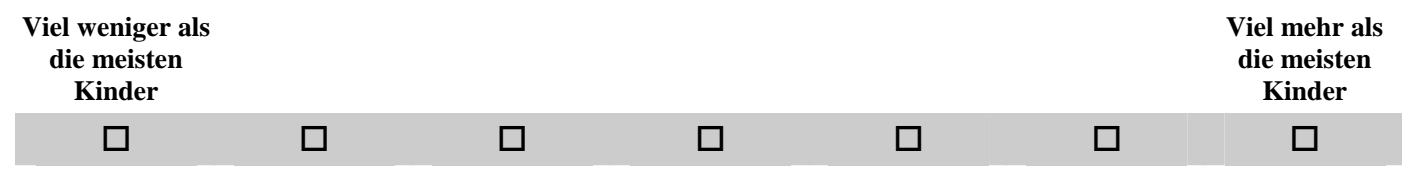

24. Inwieweit ist das Gesundheitsrisiko durch Mykotoxine (Schimmelpilzgifte) natürlich oder von Menschen verursacht?

$\begin{array}{cccccc}\begin{array}{c}\text { Natürliches } \\ \text { Risiko }\end{array} & & & & \begin{array}{c}\text { Von Menschen } \\ \text { verursachtes } \\ \text { Risiko }\end{array} \\ \square & \square & \square & \square & \square & \square\end{array}$

25. Für wie gefährlich halten Sie eine Schwächung des Immunsystems durch Mykotoxine (Schimmelpilzgifte) bei Kindern?

$\begin{array}{ccccccc}\begin{array}{c}\text { Gar nicht } \\ \text { gefährlich }\end{array} & & & & \begin{array}{c}\text { Sehr } \\ \text { gefährlich }\end{array} \\ \square & \square & \square & \square & \square & \square & \square\end{array}$

26. Wie sehr fürchten Sie eine Gesundheitsschädigung Ihres Kindes durch Mykotoxine (Schimmelpilzgifte) in Getreideprodukten?

$\begin{array}{ccccccc}\begin{array}{c}\text { Fürchte ich } \\ \text { gar nicht }\end{array} & & & & \text { Fürchte ich } \\ \text { sehr }\end{array}$




\section{Fragen zum Gesundheitsschutz vor Mykotoxinen (Schimmelpilzgiften)}

In den nächsten Fragen geht es um den Schutz der Gesundheit von Kindern vor Mykotoxinen (Schimmelpilzgiften). Wenn dabei von Ihrem Kind gesprochen wird und Sie mehrere Kinder haben, denken Sie bitte wieder an das Kind (geboren 2001-2008), auf das Sie sich auch bei den vorherigen Fragen bezogen haben. Denken Sie bei der Beantwortung der Fragen bitte immer an dieses Kind.

Bitte bedenken Sie auch: Es geht uns nicht um Fachwissen. Wir interessieren uns für Ihre persönliche Meinung. Daher gibt es keine falschen Antworten!

27. Inwieweit liegt es in Ihrer Verantwortung oder in der Verantwortung des Staates, Ihr Kind vor einer Gesundheitsgefährdung durch Mykotoxine (Schimmelpilzgifte) in Getreideprodukten zu schützen?

$\begin{array}{ccccc}\begin{array}{c}\text { Komplett in } \\ \text { meiner } \\ \text { Verantwortung }\end{array} & & & \begin{array}{c}\text { Komplett in } \\ \text { staatlicher } \\ \text { Verantwortung }\end{array} \\ \square & \square & \square & \square & \square\end{array}$

28. Inwieweit halten Sie die aktuellen gesetzlichen Regelungen (Grenzwerte) für ausreichend, um Kinder vor Mykotoxinen (Schimmelpilzgiften) zu schützen?

\begin{tabular}{|c|c|c|c|c|}
\hline $\begin{array}{l}\text { Gar nicht } \\
\text { ausreichend }\end{array}$ & & & & $\begin{array}{l}\text { Vollkommen } \\
\text { ausreichend }\end{array}$ \\
\hline$\square$ & $\square$ & $\square$ & $\square$ & $\square$ \\
\hline
\end{tabular}

29. Welche der folgenden Einrichtungen sollte Ihrer Meinung nach bei der Festlegung von

Mykotoxin- Höchstgehalten in Lebensmitteln eine wichtige Rolle spielen? Sie können hier mehrere Kästchen ankreuzen!

$\begin{array}{llc}\square \text { Landwirtschaft } & \square \text { Wissenschaft } & \square \text { Lebensmittelkonzerne } \\ \square \text { Handel } & \square \text { Politik } & \square \text { Staat/öffentliche } \\ \square \text { Verbraucherverbände } & \square \text { Europäische Union (EU) } & \text { Institutionen/Behörden } \\ \square \text { Medien } & \square \text { Verbraucher } & \end{array}$


$\Rightarrow$ Auf den nächsten Seiten werden verschiedene Möglichkeiten beschrieben, wie die Gesundheit von Kindern vor Mykotoxinen (Schimmelpilzgiften) geschützt werden kann. Sie können insgesamt achtmal zwischen drei verschiedenen Alternativen wählen: der „Möglichkeit A“, der „Möglichkeit B“ und der „Möglichkeit C“. Die „Möglichkeit C“ ist immer gleich und beschreibt die aktuelle Situation, das heißt: es werden keine zusätzlichen Maßnahmen zum Schutz von Kindern vor Mykotoxinen (Schimmelpilzgiften) ergriffen. Die „Möglichkeit A“ und die „Möglichkeit B“ beschreiben neue Maßnahmen, die den Gesundheitsschutz von Kindern vor Mykotoxinen (Schimmelpilzgiften) erhöhen würden. Es stehen dazu die folgenden Maßnahmen zu Auswahl:

- Der Staat senkt die Grenzwerte für den Gehalt von Mykotoxinen (Schimmelpilzgiften) in allen Getreideprodukten so weit, dass ein Gesundheitsrisiko für Kinder ausgeschlossen werden kann. Alle Getreideprodukte in Deutschland enthalten deutlich weniger Mykotoxine als bisher.

\section{ODER}

- Im Handel sind spezielle Getreideprodukte für Kinder erhältlich, die nur so viele Mykotoxine (Schimmelpilzgifte) enthalten, dass ein Gesundheitsrisiko für Kinder ausgeschlossen werden kann. Diese speziellen, kindgerechten Getreideprodukte sind gekennzeichnet und dadurch leicht von herkömmlichen Getreideprodukten zu unterscheiden.

Da die Vermeidung von Mykotoxinen (Schimmelpilzgiften) aber mit Kosten verbunden ist, führen beide Maßnahmen (A, B) die den Gesundheitsschutz von Kindern verbessern dazu, dass die Preise für Getreideprodukte steigen. Dadurch erhöhen sich auch Ihre monatlichen Ausgaben für Lebensmittel. Die Erhöhung Ihrer monatlichen Ausgaben für Lebensmittel kann entweder 5\%, 10\%, 15\% oder 20\% betragen. Bei der „Möglichkeit C“, bei der keine neuen Schutzmaßnahmen vor Mykotoxinen (Schimmelpilzgiften) eingeführt werden, entstehen für Sie keine zusätzlichen Kosten.

\section{Beispiel:}

Der Staat senkt die Grenzwerte für Mykotoxine (Schimmelpilzgifte) in allen Getreideprodukten so weit, dass ein Gesundheitsrisiko für Kinder ausgeschlossen werden kann.

Ihre monatlichen Ausgaben für Lebensmittel steigen um $\mathbf{1 0} \%$.

Bitte wählen Sie aus jeder Auswahl die eine Möglichkeit (A, B, oder C) aus, die Ihnen am besten gefällt und machen Sie ein Kreuz unter diese Möglichkeit.

Berücksichtigen Sie bei jeder Auswahl auch Ihre finanzielle Situation. Wenn Sie eine teure Alternative wählen, bedenken Sie, dass Sie weniger Geld für andere Dinge ausgeben können. 


\begin{tabular}{|c|c|c|}
\hline \multicolumn{3}{|c|}{ Auswahl 1: Bitte nur ein Kreuz unter eine der drei Alternativen setzen! } \\
\hline Möglichkeit A & Möglichkeit B & Möglichkeit C \\
\hline $\begin{array}{l}\text { Der Staat senkt die } \\
\text { Grenzwerte für } \\
\text { Mykotoxine } \\
\text { (Schimmelpilzgifte) in } \\
\text { allen Getreideprodukten } \\
\text { so weit, dass ein } \\
\text { Gesundheitsrisiko für } \\
\text { Kinder ausgeschlossen } \\
\text { werden kann. }\end{array}$ & $\begin{array}{l}\text { Im Handel sind spezielle } \\
\text { Getreideprodukte für } \\
\text { Kinder erhältlich, die nur } \\
\text { so viele Mykotoxine } \\
\text { (Schimmelpilzgifte) } \\
\text { enthalten, dass ein } \\
\text { Gesundheitsrisiko für } \\
\text { Kinder ausgeschlossen } \\
\text { werden kann. }\end{array}$ & $\begin{array}{l}\text { Es werden keine } \\
\text { zusätzlichen Maßnahmen } \\
\text { ergriffen, um den } \\
\text { Gesundheitsschutz von } \\
\text { Kindern vor Mykotoxinen } \\
\text { (Schimmelpilzgiften) zu } \\
\text { erhöhen. }\end{array}$ \\
\hline $\begin{array}{l}\text { Ihre monatlichen } \\
\text { Ausgaben für Lebensmittel } \\
\text { steigen um } \mathbf{5 \%} \text {. }\end{array}$ & $\begin{array}{l}\text { Ihre monatlichen } \\
\text { Ausgaben für Lebensmittel } \\
\text { steigen um } \mathbf{1 0 \%} \text {. }\end{array}$ & $\begin{array}{l}\text { Ihre monatlichen } \\
\text { Ausgaben für Lebensmittel } \\
\text { bleiben unverändert. }\end{array}$ \\
\hline
\end{tabular}

Auf der nächsten Seite geht es weiter! 


\begin{tabular}{|c|c|c|}
\hline \multicolumn{3}{|c|}{ Auswahl 2: Bitte nur ein Kreuz unter eine der drei Alternativen setzen! } \\
\hline Möglichkeit A & Möglichkeit B & Möglichkeit C \\
\hline $\begin{array}{l}\text { Im Handel sind spezielle } \\
\text { Getreideprodukte für } \\
\text { Kinder erhältlich, die nur } \\
\text { so viele Mykotoxine } \\
\text { (Schimmelpilzgifte) } \\
\text { enthalten, dass ein } \\
\text { Gesundheitsrisiko für } \\
\text { Kinder ausgeschlossen } \\
\text { werden kann. }\end{array}$ & $\begin{array}{l}\text { Der Staat senkt die } \\
\text { Grenzwerte für } \\
\text { Mykotoxine } \\
\text { (Schimmelpilzgifte) in } \\
\text { allen Getreideprodukten } \\
\text { so weit, dass ein } \\
\text { Gesundheitsrisiko für } \\
\text { Kinder ausgeschlossen } \\
\text { werden kann. }\end{array}$ & $\begin{array}{l}\text { Es werden keine } \\
\text { zusätzlichen Maßnahmen } \\
\text { ergriffen, um den } \\
\text { Gesundheitsschutz von } \\
\text { Kindern vor Mykotoxinen } \\
\text { (Schimmelpilzgiften) zu } \\
\text { erhöhen. }\end{array}$ \\
\hline $\begin{array}{l}\text { Ihre monatlichen } \\
\text { Ausgaben für Lebensmittel } \\
\text { steigen um } \mathbf{1 5 \%} \text {. }\end{array}$ & $\begin{array}{l}\text { Ihre monatlichen } \\
\text { Ausgaben für Lebensmittel } \\
\text { steigen um } \mathbf{2 0 \%} \text {. }\end{array}$ & $\begin{array}{l}\text { Ihre monatlichen } \\
\text { Ausgaben für Lebensmittel } \\
\text { bleiben unverändert. }\end{array}$ \\
\hline
\end{tabular}




\begin{tabular}{|c|c|c|}
\hline \multicolumn{3}{|c|}{ Auswahl 3: Bitte nur ein Kreuz unter eine der drei Alternativen setzen! } \\
\hline Möglichkeit A & Möglichkeit B & Möglichkeit C \\
\hline $\begin{array}{l}\text { Der Staat senkt die } \\
\text { Grenzwerte für } \\
\text { Mykotoxine } \\
\text { (Schimmelpilzgifte) in } \\
\text { allen Getreideprodukten } \\
\text { so weit, dass ein } \\
\text { Gesundheitsrisiko für } \\
\text { Kinder ausgeschlossen } \\
\text { werden kann. }\end{array}$ & $\begin{array}{l}\text { Im Handel sind spezielle } \\
\text { Getreideprodukte für } \\
\text { Kinder erhältlich, die nur } \\
\text { so viele Mykotoxine } \\
\text { (Schimmelpilzgifte) } \\
\text { enthalten, dass ein } \\
\text { Gesundheitsrisiko für } \\
\text { Kinder ausgeschlossen } \\
\text { werden kann. }\end{array}$ & $\begin{array}{l}\text { Es werden keine } \\
\text { zusätzlichen Maßnahmen } \\
\text { ergriffen, um den } \\
\text { Gesundheitsschutz von } \\
\text { Kindern vor Mykotoxinen } \\
\text { (Schimmelpilzgiften) zu } \\
\text { erhöhen. }\end{array}$ \\
\hline $\begin{array}{l}\text { Ihre monatlichen } \\
\text { Ausgaben für Lebensmittel } \\
\text { steigen um } \mathbf{2 0 \%} \text {. }\end{array}$ & $\begin{array}{l}\text { Ihre monatlichen } \\
\text { Ausgaben für Lebensmittel } \\
\text { steigen um } \mathbf{5 \%} \text {. }\end{array}$ & $\begin{array}{l}\text { Ihre monatlichen } \\
\text { Ausgaben für Lebensmittel } \\
\text { bleiben unverändert. }\end{array}$ \\
\hline
\end{tabular}

Auf der nächsten Seite geht es weiter! 


\begin{tabular}{|c|c|c|}
\hline \multicolumn{3}{|c|}{ Auswahl 4: Bitte nur ein Kreuz unter eine der drei Alternativen setzen! } \\
\hline Möglichkeit A & Möglichkeit B & Möglichkeit C \\
\hline $\begin{array}{l}\text { Im Handel sind spezielle } \\
\text { Getreideprodukte für } \\
\text { Kinder erhältlich, die nur } \\
\text { so viele Mykotoxine } \\
\text { (Schimmelpilzgifte) } \\
\text { enthalten, dass ein } \\
\text { Gesundheitsrisiko für } \\
\text { Kinder ausgeschlossen } \\
\text { werden kann. }\end{array}$ & $\begin{array}{l}\text { Der Staat senkt die } \\
\text { Grenzwerte für } \\
\text { Mykotoxine } \\
\text { (Schimmelpilzgifte) in } \\
\text { allen Getreideprodukten } \\
\text { so weit, dass ein } \\
\text { Gesundheitsrisiko für } \\
\text { Kinder ausgeschlossen } \\
\text { werden kann. }\end{array}$ & $\begin{array}{l}\text { Es werden keine } \\
\text { zusätzlichen Maßnahmen } \\
\text { ergriffen, um den } \\
\text { Gesundheitsschutz von } \\
\text { Kindern vor Mykotoxinen } \\
\text { (Schimmelpilzgiften) zu } \\
\text { erhöhen. }\end{array}$ \\
\hline $\begin{array}{l}\text { Ihre monatlichen } \\
\text { Ausgaben für Lebensmittel } \\
\text { steigen um } \mathbf{1 0 \%} \text {. }\end{array}$ & $\begin{array}{l}\text { Ihre monatlichen } \\
\text { Ausgaben für Lebensmittel } \\
\text { steigen um } \mathbf{1 5 \%} \text {. }\end{array}$ & $\begin{array}{l}\text { Ihre monatlichen } \\
\text { Ausgaben für Lebensmittel } \\
\text { bleiben unverändert. }\end{array}$ \\
\hline
\end{tabular}




\begin{tabular}{|c|c|c|}
\hline \multicolumn{3}{|c|}{ Auswahl 5: Bitte nur ein Kreuz unter eine der drei Alternativen setzen! } \\
\hline Möglichkeit A & Möglichkeit B & Möglichkeit C \\
\hline $\begin{array}{l}\text { Der Staat senkt die } \\
\text { Grenzwerte für } \\
\text { Mykotoxine } \\
\text { (Schimmelpilzgifte) in } \\
\text { allen Getreideprodukten } \\
\text { so weit, dass ein } \\
\text { Gesundheitsrisiko für } \\
\text { Kinder ausgeschlossen } \\
\text { werden kann. }\end{array}$ & $\begin{array}{l}\text { Im Handel sind spezielle } \\
\text { Getreideprodukte für } \\
\text { Kinder erhältlich, die nur } \\
\text { so viele Mykotoxine } \\
\text { (Schimmelpilzgifte) } \\
\text { enthalten, dass ein } \\
\text { Gesundheitsrisiko für } \\
\text { Kinder ausgeschlossen } \\
\text { werden kann. }\end{array}$ & $\begin{array}{l}\text { Es werden keine } \\
\text { zusätzlichen Maßnahmen } \\
\text { ergriffen, um den } \\
\text { Gesundheitsschutz von } \\
\text { Kindern vor Mykotoxinen } \\
\text { (Schimmelpilzgiften) zu } \\
\text { erhöhen. }\end{array}$ \\
\hline $\begin{array}{l}\text { Ihre monatlichen } \\
\text { Ausgaben für Lebensmittel } \\
\text { steigen um } \mathbf{1 5 \%} \text {. }\end{array}$ & $\begin{array}{l}\text { Ihre monatlichen } \\
\text { Ausgaben für Lebensmittel } \\
\text { steigen um } \mathbf{2 0 \%} \text {. }\end{array}$ & $\begin{array}{l}\text { Ihre monatlichen } \\
\text { Ausgaben für Lebensmittel } \\
\text { bleiben unverändert. }\end{array}$ \\
\hline
\end{tabular}

Auf der nächsten Seite geht es weiter! 


\begin{tabular}{|c|c|c|}
\hline \multicolumn{3}{|c|}{ Auswahl 6: Bitte nur ein Kreuz unter eine der drei Alternativen setzen! } \\
\hline Möglichkeit A & Möglichkeit B & Möglichkeit C \\
\hline $\begin{array}{l}\text { Im Handel sind spezielle } \\
\text { Getreideprodukte für } \\
\text { Kinder erhältlich, die nur } \\
\text { so viele Mykotoxine } \\
\text { (Schimmelpilzgifte) } \\
\text { enthalten, dass ein } \\
\text { Gesundheitsrisiko für } \\
\text { Kinder ausgeschlossen } \\
\text { werden kann. }\end{array}$ & $\begin{array}{l}\text { Der Staat senkt die } \\
\text { Grenzwerte für } \\
\text { Mykotoxine } \\
\text { (Schimmelpilzgifte) in } \\
\text { allen Getreideprodukten } \\
\text { so weit, dass ein } \\
\text { Gesundheitsrisiko für } \\
\text { Kinder ausgeschlossen } \\
\text { werden kann. }\end{array}$ & $\begin{array}{l}\text { Es werden keine } \\
\text { zusätzlichen Maßnahmen } \\
\text { ergriffen, um den } \\
\text { Gesundheitsschutz von } \\
\text { Kindern vor Mykotoxinen } \\
\text { (Schimmelpilzgiften) zu } \\
\text { erhöhen. }\end{array}$ \\
\hline $\begin{array}{l}\text { Ihre monatlichen } \\
\text { Ausgaben für Lebensmittel } \\
\text { steigen um } \mathbf{5 \%} \text {. }\end{array}$ & $\begin{array}{l}\text { Ihre monatlichen } \\
\text { Ausgaben für Lebensmittel } \\
\text { steigen um } \mathbf{1 0 \%} \text {. }\end{array}$ & $\begin{array}{l}\text { Ihre monatlichen } \\
\text { Ausgaben für Lebensmittel } \\
\text { bleiben unverändert. }\end{array}$ \\
\hline
\end{tabular}




\begin{tabular}{|c|c|c|}
\hline \multicolumn{3}{|c|}{ Auswahl 7: Bitte nur ein Kreuz unter eine der drei Alternativen setzen! } \\
\hline Möglichkeit A & Möglichkeit B & Möglichkeit C \\
\hline $\begin{array}{l}\text { Der Staat senkt die } \\
\text { Grenzwerte für } \\
\text { Mykotoxine } \\
\text { (Schimmelpilzgifte) in } \\
\text { allen Getreideprodukten } \\
\text { so weit, dass ein } \\
\text { Gesundheitsrisiko für } \\
\text { Kinder ausgeschlossen } \\
\text { werden kann. }\end{array}$ & $\begin{array}{l}\text { Im Handel sind spezielle } \\
\text { Getreideprodukte für } \\
\text { Kinder erhältlich, die nur } \\
\text { so viele Mykotoxine } \\
\text { (Schimmelpilzgifte) } \\
\text { enthalten, dass ein } \\
\text { Gesundheitsrisiko für } \\
\text { Kinder ausgeschlossen } \\
\text { werden kann. }\end{array}$ & $\begin{array}{l}\text { Es werden keine } \\
\text { zusätzlichen Maßnahmen } \\
\text { ergriffen, um den } \\
\text { Gesundheitsschutz von } \\
\text { Kindern vor Mykotoxinen } \\
\text { (Schimmelpilzgiften) zu } \\
\text { erhöhen. }\end{array}$ \\
\hline $\begin{array}{l}\text { Ihre monatlichen } \\
\text { Ausgaben für Lebensmittel } \\
\text { steigen um } \mathbf{1 0 \%} \text {. }\end{array}$ & $\begin{array}{l}\text { Ihre monatlichen } \\
\text { Ausgaben für Lebensmittel } \\
\text { steigen um } \mathbf{1 5 \%} \text {. }\end{array}$ & $\begin{array}{l}\text { Ihre monatlichen } \\
\text { Ausgaben für Lebensmittel } \\
\text { bleiben unverändert. }\end{array}$ \\
\hline
\end{tabular}

Gleich haben Sie es geschafft! 


\begin{tabular}{|c|c|c|}
\hline \multicolumn{3}{|c|}{ Auswahl 8: Bitte nur ein Kreuz unter eine der drei Alternativen setzen } \\
\hline Möglichkeit A & Möglichkeit B & Möglichkeit C \\
\hline $\begin{array}{l}\text { Im Handel sind spezielle } \\
\text { Getreideprodukte für } \\
\text { Kinder erhältlich, die nur } \\
\text { so viele Mykotoxine } \\
\text { (Schimmelpilzgifte) } \\
\text { enthalten, dass ein } \\
\text { Gesundheitsrisiko für } \\
\text { Kinder ausgeschlossen } \\
\text { werden kann. }\end{array}$ & $\begin{array}{l}\text { Der Staat senkt die } \\
\text { Grenzwerte für } \\
\text { Mykotoxine } \\
\text { (Schimmelpilzgifte) in } \\
\text { allen Getreideprodukten } \\
\text { so weit, dass ein } \\
\text { Gesundheitsrisiko für } \\
\text { Kinder ausgeschlossen } \\
\text { werden kann. }\end{array}$ & $\begin{array}{l}\text { Es werden keine } \\
\text { zusätzlichen Maßnahmen } \\
\text { ergriffen, um den } \\
\text { Gesundheitsschutz von } \\
\text { Kindern vor Mykotoxinen } \\
\text { (Schimmelpilzgiften) zu } \\
\text { erhöhen. }\end{array}$ \\
\hline $\begin{array}{l}\text { Ihre monatlichen } \\
\text { Ausgaben für Lebensmittel } \\
\text { steigen um } \mathbf{2 0 \%} \text {. }\end{array}$ & $\begin{array}{l}\text { Ihre monatlichen } \\
\text { Ausgaben für Lebensmittel } \\
\text { steigen um } \mathbf{5 \%} \text {. }\end{array}$ & $\begin{array}{l}\text { Ihre monatlichen } \\
\text { Ausgaben für Lebensmittel } \\
\text { bleiben unverändert. }\end{array}$ \\
\hline
\end{tabular}

\section{Fragen zur Ernährung Ihres Kindes}

Wenn jetzt von „Ihrem Kind" gesprochen wird und Sie mehrere Kinder haben, denken Sie bitte wieder an das Kind (geboren 2001-2008), auf das Sie auch die vorherigen Fragen bezogen haben.

38. Hier sind einige Aussagen zur Ernährung Ihres Kindes aufgelistet. Bitte geben Sie an, inwieweit diese Aussagen auf Sie zutreffen.

Bitte machen Sie in jeder Zeile ein Kreuz! $\begin{array}{cc}\text { Trifft } & \text { Trifft } \\ \text { gar } & \text { voll und } \\ \text { nicht zu } & \text { ganz zu }\end{array}$

Wenn ich Lebensmittel für mein Kind kaufe, achte ich darauf, dass keine künstlichen Süßstoffe enthalten sind.

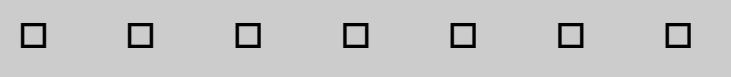

Für die Ernährung meines Kindes verwende ich überwiegend ökologisch produzierte Lebensmittel.

Wenn ich Lebensmittel für mein Kind kaufe, lese ich mir die Zutatenliste immer genau durch.

39. Wie oft isst Ihr Kind Getreideprodukte wie Nudeln oder Brot und Brötchen normalerweise?
$\square$ Nie
Bis zu einmal pro Woche
Mehrmals pro Woche, aber nicht täglich
$\square$ Einmal täglich
Zwei- bis dreimal täglich
Viermal täglich oder öfter

40. Haben Sie schon einmal Baby- und Kleinkindnahrung für Ihr Kind gekauft?
Nein, nie
Ja, selten
Ja, regelmäßig 
41. Welche Brot- und Brötchensorten isst Ihr Kind normalerweise mindestens einmal pro Woche? Sie können hier mehrere Kästchen ankreuzen!

Weizenbrot/Weizenmischbrot $\square$ Toastbrot

Roggenbrot/Roggenmischbrot

Weizenbrötchen
Vollkornbrot

Roggenbrötchen
Knäckebrot

Mehrkornbrötchen

Sonstige:

\section{Fragen zu speziellen Getreideprodukten für Kinder}

$\Rightarrow$ Information zu speziellen Getreideprodukten für Kinder: Neuerdings gibt es Bestrebungen, spezielle Getreideprodukte für Kinder im Handel anzubieten, um den Gesundheitsschutz vor Mykotoxinen (Schimmelpilzgiften) zu erhöhen. Diese Produkte sind gekennzeichnet und enthalten nur so viele Mykotoxine (Schimmelpilzgifte), dass ein Gesundheitsrisiko für Kinder ausgeschlossen werden kann. Bei den kindgerechten Getreideprodukten soll es sich um Kinder-Toastbrot, KinderVollkornbrot, Kinder-Mehrkornbrötchen und Kinder-Weizenbrötchen handeln. Uns interessiert, was Sie über diese Kinderbrote und Kinderbrötchen denken.

42. Wie beurteilen Sie folgende Aussagen zu den Kinderbroten und Kinderbrötchen?

Bitte machen Sie in jeder Zeile ein Kreuz!

$\begin{array}{cc}\text { Trifft } & \text { Trifft } \\ \text { gar } & \text { voll und } \\ \text { nicht zu } & \text { ganz zu }\end{array}$

Die Kinderbrote und Kinderbrötchen sind hilfreich, um den Gesundheitsschutz von Kindern vor Mykotoxinen (Schimmelpilzgiften) zu verbessern.

Ich wäre sicher, dass die Kinderbrote und Kinderbrötchen weniger Mykotoxine (Schimmelpilzgifte) enthalten als herkömmliche Produkte.

Ich denke, die Kinderbrote werden dort erhältlich sein, wo ich normalerweise auch Brot kaufe.

Ich denke, mein Kind wird das Kinderbrot gerne anstelle des Brotes essen, das es sonst bekommt.

\begin{tabular}{|c|c|c|c|c|c|c|c|}
\hline $\begin{array}{l}\text { Die Kinderbrote und Kinderbrötchen sind geeignet, um } \\
\text { Erkrankungen von Kindern durch Mykotoxine } \\
\text { (Schimmelpilzgifte) zu verhindern. }\end{array}$ & $\square$ & $\square$ & $\square$ & $\square$ & $\square$ & $\square$ & $\square$ \\
\hline Ich kann nicht auch noch extra ein Kinderbrot kaufen. & $\square$ & $\square$ & $\square$ & $\square$ & $\square$ & $\square$ & $\square$ \\
\hline $\begin{array}{l}\text { Ich befürchte, die Kinderbrote und Kinderbrötchen } \\
\text { werden für meine Familie zu teuer sein. }\end{array}$ & $\square$ & $\square$ & $\square$ & $\square$ & $\square$ & $\square$ & $\square$ \\
\hline $\begin{array}{l}\text { Die Kinderbrote und Kinderbrötchen tragen dazu bei, } \\
\text { dass Kinder zukünftig nur eine ungefährliche Menge an } \\
\text { Mykotoxinen (Schimmelpilzgiften) aufnehmen. }\end{array}$ & $\square$ & $\square$ & $\square$ & $\square$ & $\square$ & $\square$ & $\square$ \\
\hline $\begin{array}{l}\text { Die Kinderbrote und Kinderbrötchen sind wichtig, damit } \\
\text { ich die Gesundheit meines Kindes zukünftig besser vor } \\
\text { Mykotoxinen (Schimmelpilzgiften) schützen kann. }\end{array}$ & $\square$ & $\square$ & $\square$ & $\square$ & $\square$ & $\square$ & $\square$ \\
\hline
\end{tabular}


43. Würden Sie spezielle Kinderbrote oder Kinderbrötchen, die nur so viele Mykotoxine (Schimmelpilzgifte) enthalten, dass ein Gesundheitsrisiko für Kinder ausgeschlossen werden kann, für Ihr Kind kaufen?
$\square$ Nein
$\square$ Vielleicht
$\square \mathrm{Ja}$

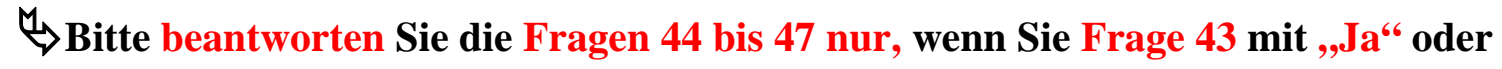
„Vielleicht" beantwortet haben! Wenn Sie Frage 43 mit „Nein“ beantwortet haben, überspringen Sie bitte Fragen 44 bis 47.

44. Wie viel zahlen Sie normalerweise für ein Toastbrot (500 g)? $€$

Wie viel wären Sie bereit mehr für ein Kinder-Toastbrot (500 g) zu zahlen, das nur so viele Mykotoxine (Schimmelpilzgifte) enthält, dass ein Gesundheitsrisiko für Ihr Kind ausgeschlossen werden kann?

Maximal: $\square+0$ Cent $\square+20$ Cent $\square+40$ Cent $\square+60$ Cent $\square+80$ Cent $\square+1,00 € \quad \square+1,20 €$

Mein Kind isst kein Toastbrot.

45. Wie viel zahlen Sie normalerweise für ein Weizenbrötchen? Cent

Wie viel wären Sie bereit mehr für ein Kinder-Weizenbrötchen zu zahlen, das nur so viele Mykotoxine (Schimmelpilzgifte) enthält, dass ein Gesundheitsrisiko für Ihr Kind ausgeschlossen werden kann?

Maximal: $\square+0$ Cent $\square+5$ Cent $\square+10$ Cent $\square+15$ Cent $\square+20$ Cent $\square+25$ Cent $\square+30$ Cent

Mein Kind isst keine Weizenbrötchen.

46. Wie viel zahlen Sie normalerweise für ein Vollkornbrot $(1 \mathrm{~kg})$ ? $€$

Wie viel wären Sie bereit mehr für ein Kinder-Vollkornbrot $(1 \mathrm{~kg})$ zu zahlen, das nur so viele Mykotoxine (Schimmelpilzgifte) enthält, dass ein Gesundheitsrisiko für Ihr Kind ausgeschlossen werden kann?

Maximal: $\square+0$ Cent $\square+40$ Cent $\square+80$ Cent $\square+1,20 € \quad \square+1,60 € \quad \square+2,00 € \quad \square+2,40 €$

Mein Kind isst kein Vollkornbrot. 
47. Wie viel zahlen Sie normalerweise für ein Mehrkornbrötchen? Cent

Wie viel wären Sie bereit mehr für ein Kinder-Mehrkornbrötchen zu zahlen, das nur so viele Mykotoxine (Schimmelpilzgifte) enthält, dass ein Gesundheitsrisiko für Ihr Kind ausgeschlossen werden kann?

Maximal: $\square+0$ Cent $\square+10$ Cent $\quad \square+20$ Cent $\quad \square+30$ Cent $\quad \square+40$ Cent $\square+50$ Cent $\quad \square+60$ Cent

Mein Kind isst keine Mehrkornbrötchen.

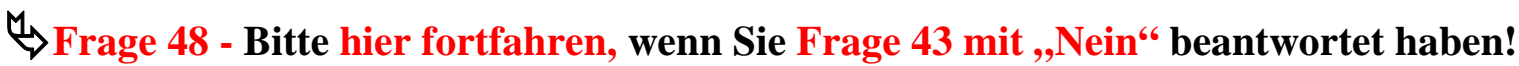

$\Rightarrow$ Stellen Sie sich nun bitte folgende Situation vor: Sie möchten ein Toastbrot kaufen, von dem auch Ihr Kind isst. In einem Supermarkt stehen die drei unten abgebildeten Toastbrote zu Auswahl.

Welches würden Sie kaufen? Bitte machen Sie ein Kreuz unter das entsprechende Toastbrot!

Dieser spezielle

Kindertoast enthält nur so viele Mykotoxine

(Schimmelpilzgifte) dass ein Gesundheitsrisiko für

Kinder ausgeschlossen werden kann.

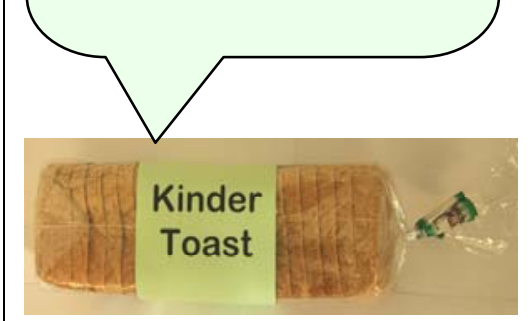

Preis: 0,94 €

\section{Die Grenzwerte für} Mykotoxine

(Schimmelpilzgifte) wurden generell gesenkt. Dieses Toastbrot enthält nur so viele Mykotoxine, dass ein Gesundheitsrisiko für Kinder ausgeschlossen werden kann.

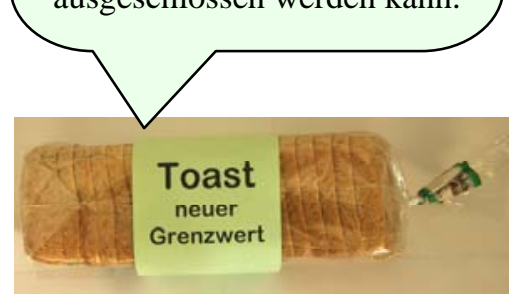

Preis: $1,08 €$
Herkömmliches Toastbrot. Es wurden keine zusätzlichen Maßnahmen ergriffen, um den Gesundheitsschutz von Kindern vor Mykotoxinen (Schimmelpilzgiften) zu erhöhen.

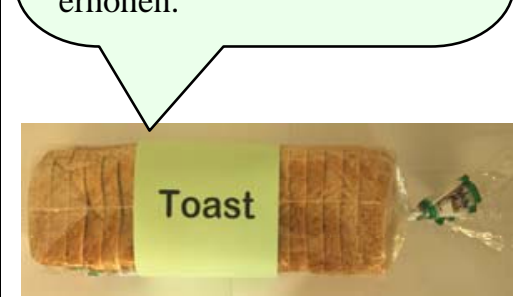

Preis: 0,65 €

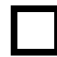

\section{Fragen zu allgemeinen Ansichten und alltäglichem Verhalten}

49. Wenn am nächsten Sonntag Bundestagswahl wäre, welche Partei würde Sie dann mit Ihrer

Zweitstimme wählen?

$\square$ CDU bzw. CSU

SPD

$\square$ FDP

$\square$ Die Linke

Bündnis 90/ Die Grünen

NPD

Andere Partei, und zwar:

Weiß nicht

Würde nicht wählen
Nicht wahlberechtigt, da keine deutsche Staatsbürgerschaft 
50. Im Folgenden finden Sie eine Liste von Aussagen. Lesen Sie bitte jeden Satz und bestimmen Sie, ob die jeweilige Aussage auf Sie zutrifft oder nicht. Trifft sie zu, machen Sie bitte ein Kreuz bei „,richtig“, ansonsten bei „falsch". Bedenken Sie bitte, dass niemand weiß, wer diesen Fragebogen ausgefüllt hat.

\begin{tabular}{|l|c|c|}
\multicolumn{1}{l}{ Falsch } & Richtig \\
\hline Im Straßenverkehr nehme ich stets Rücksicht auf die anderen Verkehrsteilnehmer. & $\square$ & $\square$ \\
\hline Ich habe schon einmal Drogen (Tabletten, Haschisch oder "ähnliches") konsumiert. & $\square$ & $\square$ \\
\hline $\begin{array}{l}\text { Meine Wut oder schlechte Laune lasse ich hin und wieder an unschuldigen oder } \\
\text { schwächeren Leuten aus. }\end{array}$ & $\square$ & $\square$ \\
\hline $\begin{array}{l}\text { Ich akzeptiere alle anderen Meinungen, auch wenn sie mit meiner eigenen nicht } \\
\text { übereinstimmen. }\end{array}$ & $\square$ & $\square$ \\
\hline Ich habe schon einmal jemanden ausgenutzt oder übers Ohr gehauen. & $\square$ & $\square$ \\
\hline In einem Gespräch lasse ich den anderen stets ausreden und höre ihm aufmerksam zu. & $\square$ & $\square$ \\
\hline Ich zögere niemals, jemandem in einer Notlage beizustehen. & $\square$ & $\square$ \\
\hline Wenn ich etwas versprochen habe, halte ich es ohne Wenn und Aber. & $\square$ & $\square$ \\
\hline Ich würde niemals auf Kosten der Allgemeinheit leben. & $\square$ & $\square$ \\
\hline $\begin{array}{l}\text { Ich bleibe immer freundlich und zuvorkommend anderen Leuten gegenüber, auch wenn } \\
\text { ich selbst gestresst bin. }\end{array}$ & $\square$ & $\square$ \\
\hline Ich habe schon einmal geliehene Sachen nicht zurückgegeben. & $\square$ & $\square$ \\
\hline Ich ernähre mich stets gesund. & $\square$ & $\square$ \\
\hline Manchmal helfe ich nur, weil ich eine Gegenleistung erwarte. & $\square$ & $\square$ \\
\hline
\end{tabular}

\section{Angaben zu Ihnen und Ihrer Familie}
51. Sie sind...
$\square$ eine Frau
ein Mann

52. In welchem Jahr sind Sie geboren?

19 $-5$

53. Wie viele Personen gehören insgesamt zu Ihrem Haushalt? Sie selbst eingeschlossen.

Anzahl Personen<smiles>[3H][Te]</smiles>

54. Wie viele Kinder unter 14 Jahren leben insgesamt in Ihrem Haushalt und wann sind diese

Kinder geboren? Anzahl Kinder unter 14 Jahren insgesamt $\infty$

\begin{tabular}{|l|l|}
\hline \multicolumn{2}{|c|}{ Geburtsjahre der Kinder unter 14 Jahren im Haushalt } \\
\hline Geburtsjahr Kind 1: & Geburtsjahr Kind 5: \\
\hline Geburtsjahr Kind 2: & Geburtsjahr Kind 6: \\
\hline Geburtsjahr Kind 3: & Geburtsjahr Kind 7: \\
\hline Geburtsjahr Kind 4: & Geburtsjahr Kind 8: \\
\hline
\end{tabular}


55. Welchen höchsten Schulabschluss haben Sie und Ihr(e) Partner(in)?

\begin{tabular}{|l|c|c|}
\hline & Sie selbst: & Ihr(e) Partner(in): \\
\hline Bin noch Schüler/Schülerin & $\square$ & $\square$ \\
\hline Schule ohne Abschluss beendet & $\square$ & $\square$ \\
\hline $\begin{array}{l}\text { Volks-/Hauptschulabschluss, Polytechnische Oberschule } \\
\text { mit Abschluss 8. Klasse oder 9. Klasse }\end{array}$ & $\square$ & $\square$ \\
\hline $\begin{array}{l}\text { Mittlere Reife, Realschulabschluss, Polytechnische } \\
\text { Oberschule Abschluss 10. Klasse }\end{array}$ & $\square$ & $\square$ \\
\hline Fachhochschulreife (Abschluss einer Fachoberschule) & $\square$ & $\square$ \\
\hline $\begin{array}{l}\text { Abitur (Hochschulreife) bzw. erweiterte Oberschule mit } \\
\text { Abschluss 12. Klasse }\end{array}$ & $\square$ & $\square$ \\
\hline Fachhochschulabschluss & $\square$ & $\square$ \\
\hline Universitätsabschluss, Hochschulabschluss & $\square$ & $\square$ \\
\hline Anderer Schulabschluss & $\square$ & $\square$ \\
\hline
\end{tabular}

56. Wie hoch ist das monatliche Netto-Haushaltseinkommen Ihres Haushalts insgesamt? Gemeint ist die Summe, die alle Personen in Ihrem Haushalt (siehe Frage 53), nach Abzug der Steuern und Sozialabgaben zur Verfügung haben (einschließlich Erziehungs- und Kindergeld etc.)?

Netto-Haushalteinkommen Ihres Haushalts in Euro

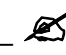

$\Rightarrow$ Falls Sie Frage 56 nicht beantwortet haben, bitten wir Sie Frage 57 zu beantworten. Bedenken

Sie bitte, dass niemand weiß, wer diesen Fragebogen ausgefüllt hat!

57. Wie hoch ist das monatliche Netto-Haushaltseinkommen Ihres Haushalts insgesamt? Gemeint ist die Summe, die alle Personen in Ihrem Haushalt (siehe Frage 53), nach Abzug der Steuern und Sozialabgaben zur Verfügung haben (einschließlich Erziehungs- und Kindergeld etc.)?
$\square$ Unter $1000 €$
$\square 3000$ bis unter $4000 €$
$\square 6000$ bis unter $7000 €$
$\square 1000$ bis unter $2000 €$
$\square 4000$ bis unter $5000 €$
$\square 7000 €$ oder mehr
$\square 2000$ bis unter $3000 €$
$\square 5000$ bis unter $6000 €$

58. Haben Sie oder eine andere erwachsene Person in Ihrem Haushalt beruflich mit dem Themen Ernährung oder Gesundheit zu tun?

$\square$ Nein

$\square \mathrm{Ja}$

59. Was aus der nachstehenden Aufzählung trifft auf Sie zu? Ich bin zurzeit...

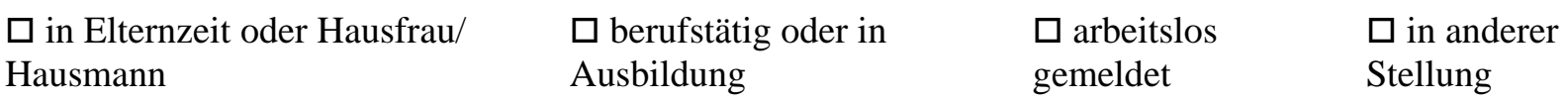

60. In welchem Landkreis wohnen Sie? 
61. Wie viele Einwohner hat Ihr Wohnort ungefähr?

$\square$ Bis unter 500 Einwohner

$\square 500$ bis unter 1000 Einwohner

$\square 1000$ bis unter 1500 Einwohner

$\square 1500$ bis unter 5000 Einwohner $\square 5000$ bis unter 10 000Einwohner

$\square 10000$ bis unter 50000 Einwohner

$\square 50000$ bis unter 100000 Einwohner

$\square 100000$ Einwohner oder mehr

Gerne können Sie hier Kommentare zum Fragebogen und zu den Themen „Schimmelpilzgifte“ und „Kinderbrot“ aufschreiben:

\section{Vielen Dank für Ihre Mitarbeit!}




\section{Lebenslauf}

Niens, Christine geb. am 26.02.1981 in Wolfenbüttel

E-Mail: cniens@uni-goettingen.de

seit 06.2009

Wissenschaftliche Mitarbeiterin am Department für Agrarökonomie und Rurale Entwicklung der Universität Göttingen

Abteilung Umwelt- und Ressourcenökonomik

Projekt: „Qualitätsgerechte Pflanzenproduktion unter veränderten Rahmenbedingungen: Mykotoxine im Kontext von Produktion, Qualität und Verarbeitung: Implementierung eines kohärenten Managementsystems für Mykotoxinrisiken an Getreideprodukten in Niedersachsen: Effizienzanalyse“

09.2009-07.2013 Promotionsstudentin am Department für Agrarökonomie und Rurale Entwicklung der Universität Göttingen

Abteilung Umwelt- und Ressourcenökonomik

Titel der Dissertation: „Mykotoxine und Kindergesundheit:

Elterliche Risikowahrnehmung und neue Ansätze für das

Risikomanagement"

06.2008-05.2009 Wissenschaftliche Hilfskraft am Zentrum für Landwirtschaft und Umwelt der Universität Göttingen

Projekt: Agrarumweltmaßnahmen in Zeiten von Klimawandel und steigenden Preisen von Agrarrohstoffen Edmund Rehwinkel-Stiftung der Landwirtschaftlichen Rentenbank

01.2008 - 03.2008 Interviewerin am Zentrum für Landwirtschaft und Umwelt der Universität Göttingen im Rahmen des Projekts „Ergebnisorientierte Honorierung ökologischer Leistungen der Landwirtschaft“

10.2001 - 09.2007 Studium der Sozialwissenschaften an der Georg - August Universität Göttingen 


\section{Erklärung}

Hiermit erkläre ich, dass diese Arbeit weder in gleicher noch in ähnlicher Form bereits anderen Prüfungsbehörden vorgelegen hat.

Weiter erkläre ich, dass ich mich an keiner anderen Hochschule um einen Doktorgrad beworben habe.

Göttingen, den 08. Mai 2013

\section{Christive Vieus}

Hiermit erkläre ich eidesstattlich, dass diese Dissertation selbständig und ohne unerlaubte Hilfe angefertigt wurde.

Göttingen, den 08. Mai 2013

Christive Vieus 